University of Tennessee Health Science Center

UTHSC Digital Commons

\title{
Esthetic Evaluation of Edgewise Orthodontic Treatment in Matched Class II, Division 1 Subjects, with and without a MARA
}

Kelly-Gwynne Mason Fergus

University of Tennessee Health Science Center

Follow this and additional works at: https://dc.uthsc.edu/dissertations

Part of the Orthodontics and Orthodontology Commons

\section{Recommended Citation}

Fergus, Kelly-Gwynne Mason, "Esthetic Evaluation of Edgewise Orthodontic Treatment in Matched Class II, Division 1 Subjects, with and without a MARA" (2008). Theses and Dissertations (ETD). Paper 83. http://dx.doi.org/10.21007/etd.cghs.2008.0091. 


\title{
Esthetic Evaluation of Edgewise Orthodontic Treatment in Matched Class II, Division 1 Subjects, with and without a MARA
}

\begin{abstract}
Orthodontics' concern about facial esthetics has motivated the investigation of treatment options, and their effects, in hopes of discerning the optimal treatment for patients, both esthetically and functionally. In our efforts to determine the optimal treatment option for patients with Class II, division 1 malocclusions, we examined two current treatment methods' effects on facial esthetics, namely (1) orthopedic functional therapy using a MARA in combination with Edgewise appliances and (2) Edgewise mechanics alone. This study was a retrospective esthetic evaluation of profile silhouettes (prior to and following orthodontic treatment) of 30 consecutively treated American white adolescents with Class II, division 1 malocclusions treated using Edgewise mechanics in conjunction with a MARA. These were compared to a matched sample treated with Edgewise mechanics alone. Subjects in the two treatment groups were matched, on a one-to-one basis, for demographic and cephalometric variables (SNA, SNB, ANB, FMA) to ensure comparability in the nature and severity of the malocclusions. The question was whether the use of a MARA in combination with Edgewise mechanics yielded a more esthetic facial profile than Edgewise treatment alone. Lay people, graduate orthodontic students, and experienced orthodontists rated the level of profile attractiveness before and after orthodontic treatment. Factorial ANOVA models were used to examine the effects (and interactions among) (1) the patient's sex, (2) sex of the observer, and (3) the three groups of observers prior to and after treatment. Male observers, regardless of group, scored the patients' faces as more esthetic (higher VAS score) than did the female observers $(P<0.0001)$, though a sex difference, if any, seems to depend on the specific individuals in the sample. The ANOVA tests of VAS scores also showed a highly significant difference in observer groups ( $P$ $<0.0001$ ) with the experienced orthodontists scoring the profiles higher (more esthetic) than orthodontic residents or lay people. From a three-way ANOVA evaluating posttreatment VAS scores, the two treatments were esthetically indistinguishable statistically $(P=0.0808)$, having virtually identical median VAS scores ( $=44$ with a MARA, $=42$ without a MARA), as well as identical amounts of improvement when comparing the median pre- and posttreatment VAS scores (both increasing 10 points on average). Though marginally-significant differences were found in ANB after treatment $(P=0.03)$, similar esthetic endpoints were achieved regardless of treatment protocol. In summary, (1) both treatment protocols produced an improved level of profile attractiveness at the end of treatment, and (2) there was no difference between the two groups in the perceived amounts of profile change with treatment. MARA treatment in combination with Edgewise mechanics has measurable benefits, (1) it enhances mandibular growth and (2) it reduces ANB by moving B Point forward rather than restraining maxillary growth. It seems, however, that integumental profiles, along with variations in lip dimensions and other features outside the orthodontist's control, converge to obscure the skeletodental corrections, at least to readilydiscernible systematic extents.

\section{Document Type}

Thesis
\end{abstract}

\section{Degree Name}

Master of Dental Science (MDS)

\section{Program}

Orthodontics

Research Advisor

Edward F. Harris, Ph.D. 


\section{Keywords}

esthetic; esthetic evaluation; orthodontic treatment; orthodontics; Class II, Division 1; MARA;

\section{Subject Categories}

Dentistry | Medicine and Health Sciences | Orthodontics and Orthodontology 


\title{
ESTHETIC EVALUATION OF EDGEWISE ORTHODONTIC TREATMENT IN MATCHED CLASS II, DIVISION 1 SUBJECTS, WITH AND WITHOUT A MARA
}

\author{
A Thesis \\ Presented for \\ The Graduate Studies Council \\ The University of Tennessee \\ Health Science Center
}

\author{
In Partial Fulfillment \\ Of the Requirements for the Degree \\ Master of Dental Science \\ From The University of Tennessee
}

By

Kelly-Gwynne Mason Fergus, D.D.S.

May 2008 
Copyright ( 2008 by Kelly-Gwynne Mason Fergus All rights reserved 


\section{ACKNOWLEDGEMENTS}

I would like to thank my husband, Jay, for his unfailing love and support during the writing of this thesis, my mom and dad, who have been on this 29 year journey with me, and my many friends and family for their thoughts, prayers, and never ending encouragement through this time. I would like to thank Dr. Edward Harris for his expertise, continued guidance and leadership. Without his help this thesis would not be possible. I would also like to thank Dr. Joe Wasson and Dr. Jere Yates for serving on my committee. Their direction and support was invaluable. 


\begin{abstract}
Orthodontics' concern about facial esthetics has motivated the investigation of treatment options, and their effects, in hopes of discerning the optimal treatment for patients, both esthetically and functionally. In our efforts to determine the optimal treatment option for patients with Class II, division 1 malocclusions, we examined two current treatment methods' effects on facial esthetics, namely (1) orthopedic functional therapy using a MARA in combination with Edgewise appliances and (2) Edgewise mechanics alone. This study was a retrospective esthetic evaluation of profile silhouettes (prior to and following orthodontic treatment) of 30 consecutively treated American white adolescents with Class II, division 1 malocclusions treated using Edgewise mechanics in conjunction with a MARA. These were compared to a matched sample treated with Edgewise mechanics alone. Subjects in the two treatment groups were matched, on a one-to-one basis, for demographic and cephalometric variables (SNA, SNB, ANB, FMA) to ensure comparability in the nature and severity of the malocclusions. The question was whether the use of a MARA in combination with Edgewise mechanics yielded a more esthetic facial profile than Edgewise treatment alone. Lay people, graduate orthodontic students, and experienced orthodontists rated the level of profile attractiveness before and after orthodontic treatment. Factorial ANOVA models were used to examine the effects (and interactions among) (1) the patient's sex, (2) sex of the observer, and (3) the three groups of observers prior to and after treatment. Male observers, regardless of group, scored the patients' faces as more esthetic (higher VAS score) than did the female observers $(\mathrm{P}<0.0001)$, though a sex difference, if any, seems to depend on the specific individuals in the sample. The ANOVA tests of VAS scores also showed a highly significant difference in observer groups $(\mathrm{P}<$ 0.0001 ) with the experienced orthodontists scoring the profiles higher (more esthetic) than orthodontic residents or lay people. From a three-way ANOVA evaluating posttreatment VAS scores, the two treatments were esthetically indistinguishable statistically $(\mathrm{P}=0.0808)$, having virtually identical median VAS scores $(\overline{\mathrm{X}}=44$ with a MARA, $\overline{\mathrm{X}}=42$ without a MARA), as well as identical amounts of improvement when comparing the median pre- and posttreatment VAS scores (both increasing 10 points on average). Though marginallysignificant differences were found in ANB after treatment $(P=0.03)$, similar esthetic endpoints were achieved regardless of treatment protocol. In summary, (1) both treatment protocols produced an improved level of profile attractiveness at the end of treatment, and (2) there was no difference between the two groups in the perceived amounts of profile change with treatment. MARA treatment in combination with Edgewise mechanics has measurable benefits, (1) it enhances mandibular growth and (2) it reduces ANB by moving B Point forward rather than restraining maxillary growth. It seems, however, that integumental profiles,
\end{abstract}


along with variations in lip dimensions and other features outside the orthodontist's control, converge to obscure the skeletodental corrections, at least to readily-discernible systematic extents. 


\section{TABLE OF CONTENTS}

Chapter

Page

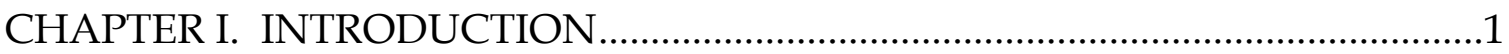

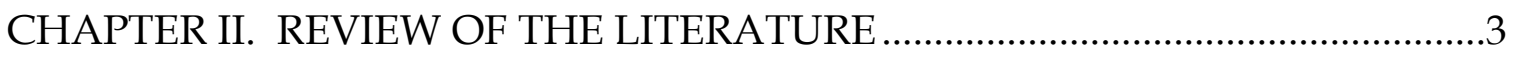

Facial Esthetics, Beauty and Attractiveness.....................................................

Historical Perspectives of Esthetic Ideals .................................................

Artistic Guidelines ....................................................................

Anthropometric Guidelines.........................................................

Cephalometric Guidelines ...................................................18

Philosophical Debate ………………………………..........................24

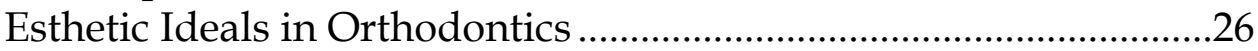

Significance in Orthodontics ............................................................2.

Previous Esthetic Evaluations in Orthodontics .................................28

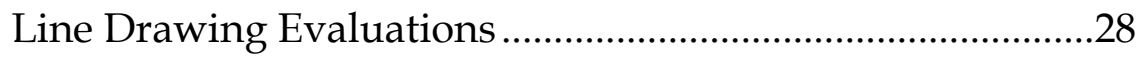

Photographic Evaluations.....................................................29

Silhouette Evaluations..............................................................31

Observer Panel Compositions.......................................................34

Measuring Techniques ...............................................................34

Profile Attractiveness Following Functional Therapy............37

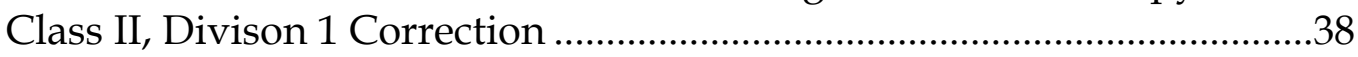

Standard Edgewise Mechanics ...........................................................38

Mandibular Anterior Repositioning Appliance ………......................41

Background .............................................................................41

Skeletodental and Cephalometric Findings ............................49

CHAPTER III. MATERIALS AND METHODS …………....................................50

Research Design .....................................................................................5

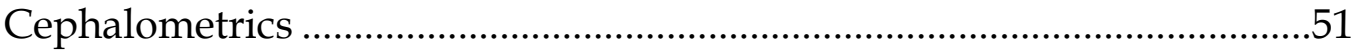

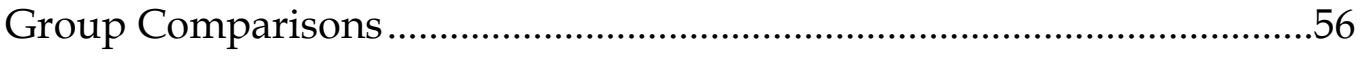

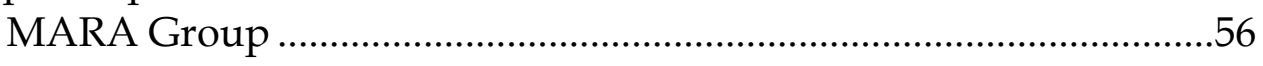

Diagnostic Considerations........................................................56

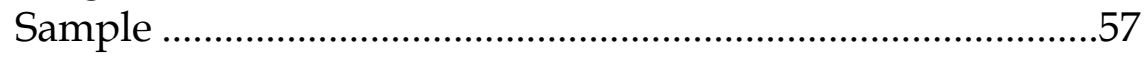

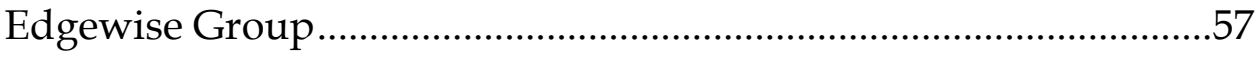

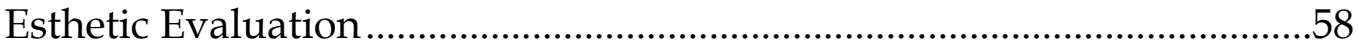

Silhouette Evaluation .........................................................................58

Observer Panel Compositions..........................................................59

Measuring Technique.........................................................................62

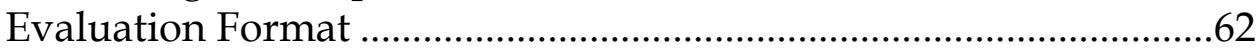

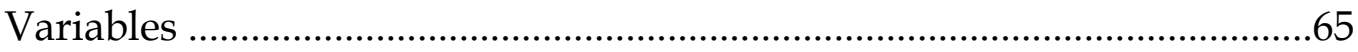


Integumental Landmarks ...................................................................65

Cephalometric Landmarks .................................................................65

Cephalometric Angles ........................................................................

Statistical Methods ..................................................................................

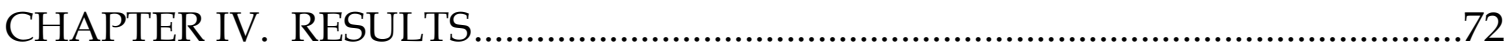

Evaluation of the Silhouettes at the Pretreatment Examination..................72

Evaluation of the Silhouettes at the Posttreatment Examination ................77

Treatment Effects Evaluated at Posttreatment .............................................8

Treatment Changes ........................................................................................ 80

Comparing Pretreatment and Posttreatment VAS Scores .................80

Observers' Perceptions of Pretreatment and Posttreatment

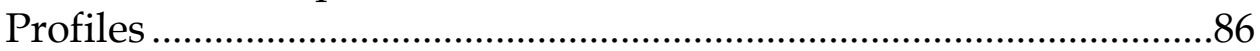

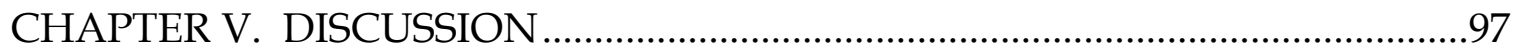

Cephalometric Influences..........................................................................97

Sex and Panel Background Influences........................................................98

Limitations in Esthetic Improvement .........................................................99

Comparison of Previous Esthetic Evaluations with Functional

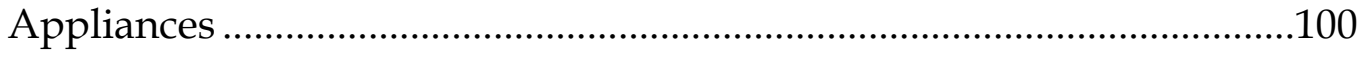

CHPATER VI. SUMMARY AND CONCLUSIONS............................................102

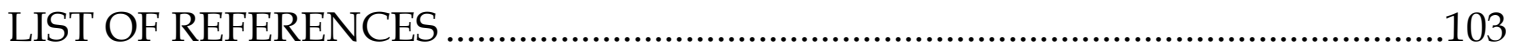

APPENDIX. SUBJECTS' SILHOUETTES ……………….....................................113

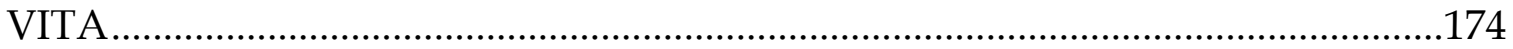




\section{LIST OF TABLES}

Table

1. Results of paired t-tests for cephalometric differences at the pretreatment examination

2. Results of paired $t$-tests for cephalometric differences at the posttreatment examination

3. Results of paired t-tests for cephalometric differences for the in-treatment changes

4. Results of ANOVA tests of the pretreatment VAS scores .73

5. Results of ANOVA tests of the posttreatment VAS scores .78

6. Results of three-way ANOVA evaluating posttreatment VAS scores, with observer sex in the model

7. Results of two-way ANOVA evaluating posttreatment VAS scores among female observers alone

8. Results of two-way ANOVA evaluating posttreatment VAS scores among male observers alone

9. Evaluation of the changes in VAS scores (male observers alone)

10. Evaluation of the changes in VAS scores (female observers alone).

11. Results of four-way ANOVA evaluating the signed VAS changes during treatment.. 


\section{LIST OF FIGURES}

Figure

1. The so-called Bartlett Head of Aphrodite.............................................................

2. A replica of Phidias's Athena Parthenos ............................................................6

3. A Roman copy of Polykleitos' Doryphoros, also known as The Lance Bearer, originally created during the fifth century B.C. .....................................

4. An example of facial trisection, as originally described by Vitruvius (ca. 70-ca. 25)

5. Leonardo da Vinci's Vitruvian Man (ca. 1485), also known as the Canon of Proportions or the Proportions of Man

6. Albrecht Dürer's depiction of (a) retroclined and (b) proclined facial contours from the angle formed between the vertical and horizontal axes of his coordinate system

7. Vertical and sagittal relationships of the facial profile by Leonardo (a) and Dürer (b)

8. A Roman copy of Apollo Belvedere, representing one of the greatest legacies of Greek art, originally thought to have been produced in bronze by the Athenian sculptor Leochares between 350 to 325 B.C.

9. The Aphrodite of Milo, better known as Venus de Milo, an ancient Greek statue presently on display at the Louve Museum in Paris dates back to 130-90 B.C.

10. Donatello's Saint George (ca. 1415-1417) ………………...............................15

11. Michelangelo's David (ca. 1501-1504) is a 17 foot marble statue portraying the Biblical King David preparing for battle against Goliah ......16

12. Example of Camper's "facial angle," that was based on comparative anatomy

13. Representation of Burstone's five contour angles 
14. Representation of Burstone's 10 inclination angles

15. Schematic tracing of a lateral cephalogram showing construction of Ricketts's esthetic plane

16. Merrifield's $Z$ angle, the inferior-posterior angle between Frankfort Horizontal (Porion and Orbitale) and Merrifield's profile line (a line drawn through the most prominent lip and the tangent of the soft tissue chin).

17. Schematic tracing of a lateral cephalogram showing construction of Frankfort Horizontal

18. Example of a Visual Analogue Scale (VAS), a horizontal line, $100 \mathrm{~mm}$ in length, anchored by word descriptors at each end.

19. Schematic tracing of a lateral cephalogram showing construction of FMA

20. Schematic tracing of a lateral cephalogram showing construction of the angle $(\theta)$ of the mandibular incisor and the mandibular plane (IMPA) .......40

21. Schematic tracing of a lateral cephalogram showing construction of FMIA angle $(\theta)$.

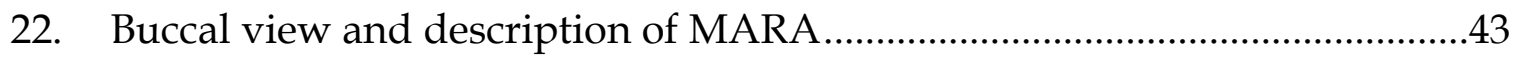

23. Frontal view and description of MARA.......................................................4

24. Schematic views of how the MARA works .....................................................

25. (A.) Patient with a typical Class II profile. (B.) Patient with lower jaw positioned forward and edge-to-edge with MARA.

26. Speculative changes produced by the MARA

27. Schematic showing locations of the integumental landmarks used in this study .60

28. Example of the VAS used in Part 1 of the esthetic evaluation .63

29. Example of the VAS used in Part 2 of the esthetic evaluation .64 
30. Cephalometric diagram showing locations of the skeletodental landmarks used in this study.

31. Schematic tracing of a lateral cephalogram showing construction of the SNA angle $(\theta)$....

32. Schematic tracing of a lateral cephalogram showing construction of the SNB angle $(\theta)$.

33. Schematic tracing of a lateral cephalogram showing construction of the angle ANB $(\theta)$.

34. Mean VAS scores, by sex and group of observers, at the pretreatment examination

35. Bar charts of the VAS scores at the start of treatment, by rater group and sex (i.e., sex of the rater) .75

36. Bar charts of the pretreatment VAS scores, by treatment group .76

37. Mean VAS scores, by sex and group of observers, at the posttreatment examination

38. Bar charts of the VAS scores at the end of treatment, by rater group and sex (i.e., sex of the rater)

39. Mean VAS scores at posttreatment, by group of observers, depending on whether the observers were males or females.

40. Bar charts of the change in VAS scores, by background of the observer.

41. Distribution of in-treatment changes in VAS scores

42. Bar charts of the VAS scores, by treatment group, at the posttreatment examination

43. Bar charts of the change in VAS scores, by sex of the patient, as perceived by the raters

44. Bar charts of the in-treatment change in VAS scores, partitioned by sex of the observer. .95 


\section{CHAPTER I INTRODUCTION}

Facial esthetics is an important personal and social concern. Attractive facial appearances are judged to possess more socially desirable personality traits (e.g., Shaw 1981), and favorable facial esthetics are related to psychosocial wellbeing by children, young adults, and parents (Shaw 1981; Shaw et al. 1985; Birkeland et al. 2000). In addition, parents believe their child would become better liked, more successful, and overall more attractive because of esthetic improvements coincident with orthodontic treatment (Shaw et al. 1979). Hence, facial esthetics is an important concern within the specialty of orthodontics.

Considerations involving orthodontic treatment effects on facial esthetics have directed the progression of the orthodontic specialty for the past century. Calvin Case (1922) was one of the first orthodontists to stress the importance of the facial profile as being an imperative guide for determining optimal orthodontic treatment; many others have supported his claim in hopes of instituting treatment options that improve the facial attractiveness of patients (Holdaway 1956; Burstone 1958; Ricketts 1960; Merrifield 1966).

The effects of orthodontic therapy on facial esthetics has been a long-term concern within the orthodontic community (Angle 1900; Wuerpel 1937; Herzburg 1952; Burstone 1958; King 1960; Subtelny 1961; Hambleton 1963; Spyropoulos and Halazonetis 2001), but these effects also are a concern of orthodontic patients and their parents (Shaw et al. 1979; Shaw 1981; Shaw et al. 1985; Vig et al. 1999; Birkeland et al. 2000). Indeed, orthodontic treatment is sought for one of three reasons, namely the presence of facial disharmony, the malalignment of teeth, or some combination of these two problems (Vig et al. 1999). Therefore, maximizing facial esthetics is a necessary consideration during orthodontic treatment planning.

There are several treatments currently used in the correction of Class II malocclusions, including a variety of extraoral traction devices (e.g., facebows and headgears), dentoalveolar distalizing appliances (e.g., Pendulum/Pendex appliance and the Distal Jet), arch expansion appliances (e.g., the rapid maxillary expander), the extraction of teeth, orthognathic surgery, temporary anchorage devices (TADs), and functional orthopedic appliances (e.g., the Activator, the Bionator, the Fränkel appliance, the Herbst appliance, the twin block appliance, and the MARA) (Graber et al. 2005). A small number of evaluations have been made regarding a few of the previously mentioned Class II treatments and facial attractiveness (Phillips et al. 1992; O'Neill et al. 2000; Shell and Woods 2003, 2004; Stock et al. 2006). However the MARA, the most recent fixed functional 
appliance to become commercially available, has never been evaluated for its esthetic effects on the facial profile.

The present study focused on two treatment methods of Class II, division 1 malocclusions, namely (1) orthopedic functional therapy using a MARA in combination with Edgewise appliances and (2) Edgewise mechanics alone. This study investigated the perceived posttreatment facial esthetics of American white adolescents with Class II, division 1 malocclusions treated by one of these two methods. Lay people, graduate orthodontic students and experienced orthodontists were asked to rate the level of profile attractiveness before and after orthodontic treatment. Objectives of the study were:

1. to determine whether each group had an improved level of profile attractiveness following treatment;

2. to determine whether there was a difference between the two groups in the perceived amounts of profile change following treatment;

3. to compare the perceptions of the three observation panels; and

4. to compare the perceptions of female and male observers. 


\section{CHAPTER II \\ REVIEW OF THE LITERATURE}

\section{Facial Esthetics, Beauty and Attractiveness}

\section{Historical Perspectives of Esthetic Ideals}

Esthetics, derived from the Greek word aisthētikos, originally described sensory perception or the combination of characteristics that give pleasure to the senses. It was Alexander Gottlieb Baumgarten, an 18th century German philosopher, who re-examined the ancient use of the term esthetics and first coined its meaning of physical or natural beauty as it is used today. Baumgarten is also accredited with establishing esthetics as a distinct field within philosophy (Baumgarten 1989).

Knowing the influence the appearance of the face can have upon an individual, it is no surprise that artists and others throughout the centuries have sought to find a principle from which esthetics ideals and facial attractiveness are based (Angle 1900). Many artistic, anthropometric and cephalometric guidelines for esthetic ideals have historically been presented.

\section{Artistic Guidelines}

Ancient Egyptians were possibly the first to describe ideal facial and bodily proportions in grid or mathematical form, though most of the earliest theories of beauty date back to the pre-Socratic period in the works of ancient Greek artists and philosophers. Sculptures made during this period conformed to established proportions of beauty, as in the so-called Bartlett Head of Aphrodite sculpture (Figure 1) that exhibited what the ancient Greek civilization percieved to represent the ideal facial proportions. The classic Greek profile would be considered orthognathic within orthodontic concepts with an undulating upper lip and slight lower lip curl (Peck and Peck 1970).

Believing true beauty displayed harmony and that harmony was 'the due observance of proportions,' it was adopted during the Golden Age of Greece that harmonious proportions were fixed quantities (Peck and Peck 1970, p. 286). Phidias (ca. 480 B.C.-ca. 430 B.C.), a great ancient Greek sculptor, architect and painter during the fifth century B.C., is credited for establishing the 'golden' or 'divine' ratio, represented by the Greek letter $\Phi$ (phi) after Phidias, that best 


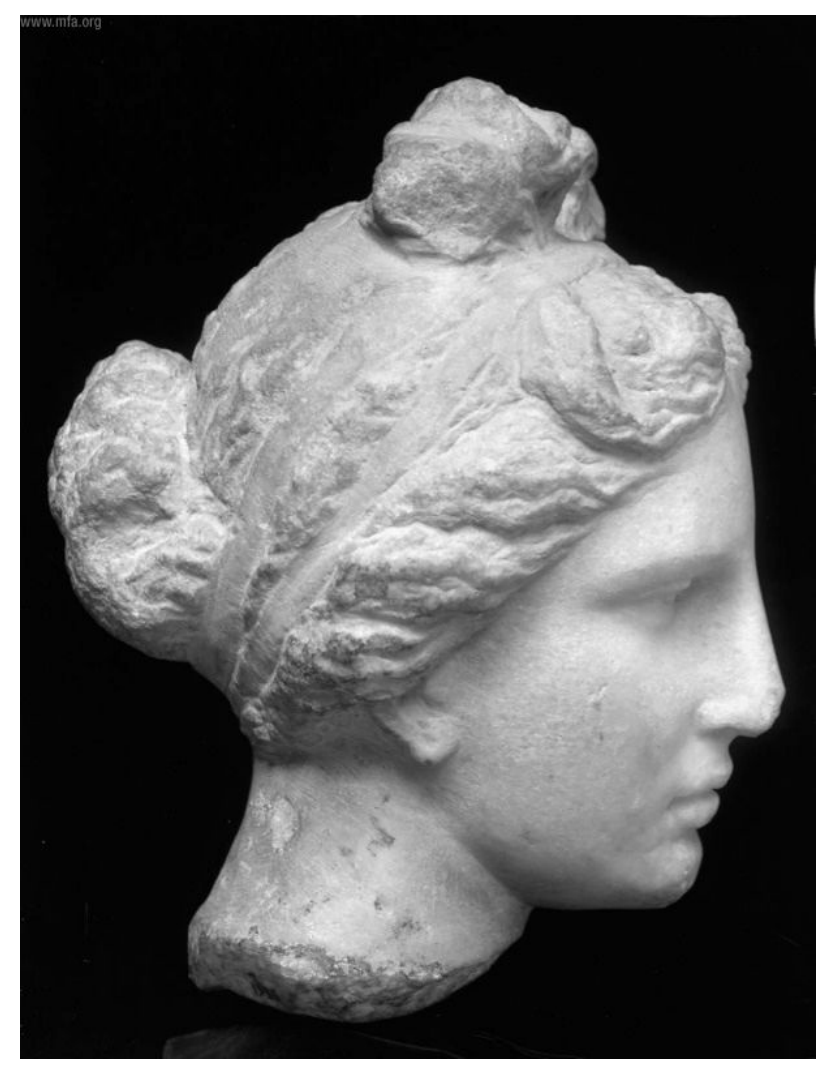

Figure 1. The so-called Bartlett Head of Aphrodite.

Reprinted by permission from the Museum of Fine Arts, Boston.

Edler RJ. Background considerations to facial aesthetics. J Orthod 2001;28:159-68. 
displays an esthetically pleasing relationship of horizontal and vertical structures. His greatest statues that display his proportional ideals of beauty include the Statue of Zeus at Olympia and Athena Parthenos (Figure 2) (Ricketts 1982).

Another well renowned sculpture from fourth century B.C. representing esthetic ideals is The Doryphoros, also known as The Lance Bearer or Spear-Bearer (Figure 3). It was sculpted by Polykleitos (ca. 450 B.C.-ca. 420 B.C.), who was not only an ancient Greek sculptor but also a theoretician and philosopher. The Doryphoros is believed to be designed according to Polykleitos's Canon, his treatise of esthetic theories and ideal ratios of the human physique. For example, the statue's head and face together comprise two of fifteen equal vertical segments by which the human body can be divided, demonstrating perfect balance and beauty. Though The Doryphoros (Figure 3) is believed to be a representation of Polykleitos' Canon historical documentation of this has never be found (Lapatin 1997).

Galen (ca. 130-ca. 200 A.D.), a Greek physician and philospher, recognized that eariler Greek sculptors, such as Polykleitos, created their art as expressions of perfect visual examples of beauty and symmetra, meaning symmetry in the human form, according to the mean. The following statement was translated from Galen's writings summarizing Polykleitos's Canon: "Modellers, sculptors, painters, and, indeed, image-makers in general, paint or model the most beautiful likenesses in each case (that is, the most beautiful man, horse, cow or lion), by observing the mean in that case. And one might comment upon a certain statue, the one called the Canon of Polykleitos [believed to be, The Doryphoros] since it received this name from its having a precise commensurability of all the parts to one another" (Stewart 1978, p. 125). Galen suggested that Polykleitos had strived to display perfection and beauty in his art often by displaying the average likeness of man.

The esthetic recommendations made by Phidias, Polykleitos and others were later copied and modified by the Romans, who presented very few original esthetic ideals. The most significant original contribution made by the Romans was from Vitruvius (ca. 80 B.C.-ca. 25 B.C.), an architect most known for writing De Architectura, also known as The Ten Books on Architecture. In perfecting the art of architecutural structures, Vitruvius also developed proportional theories of what he believed to be the greatest work of art: the human body. He is credited with developing the concept of facial trisection (Figure 4) that is still followed by orthognathic surgeons and taught in modern texts (e.g., Powell and Humphries 1984) as an esthetically pleasing face. 


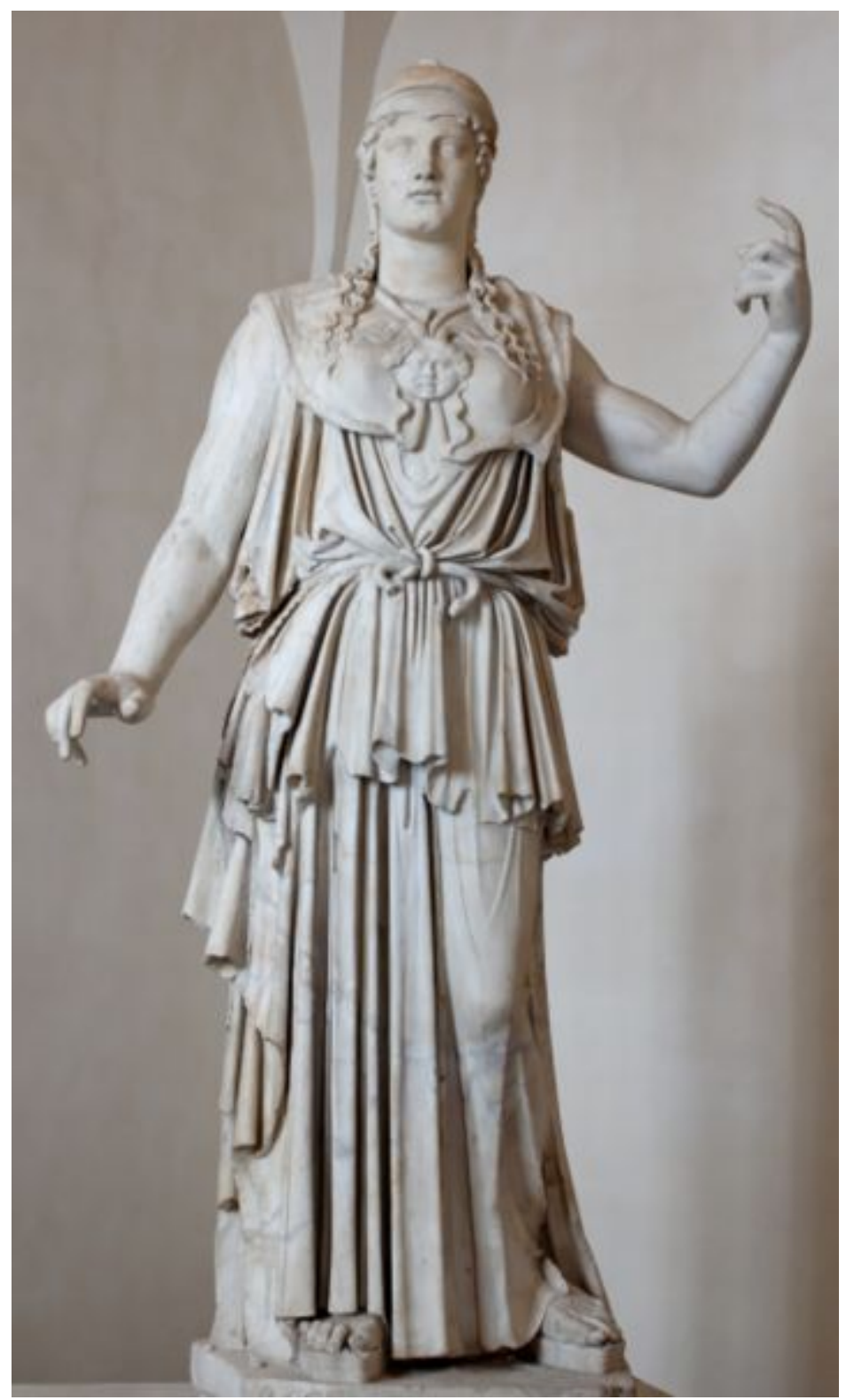

Figure 2. A replica of Phidias's Athena Parthenos.

Reprinted by permission from Wikimedia Commons. http://en.wikipedia.org/wiki/Image:Athena_Parthenos_ Altemps_Inv8622.jpg. Accessed August 28, 2007. 


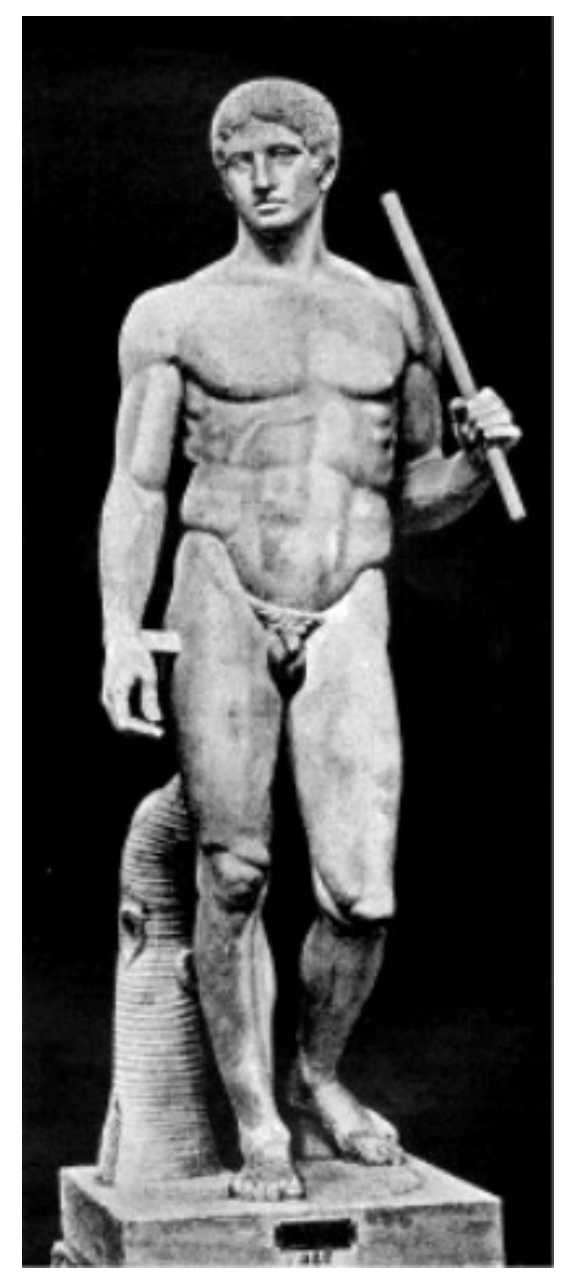

Figure 3. A Roman copy of Polykleitos' Doryphoros, also known as The Lance Bearer, originally created during the fifth century B.C.

Reprinted by permission from Wikimedia ${ }^{\circledR}$. http://en.wikipedia.org/ wiki/Image:Dorypho ros.jpg. Accessed August 29, 2007. 


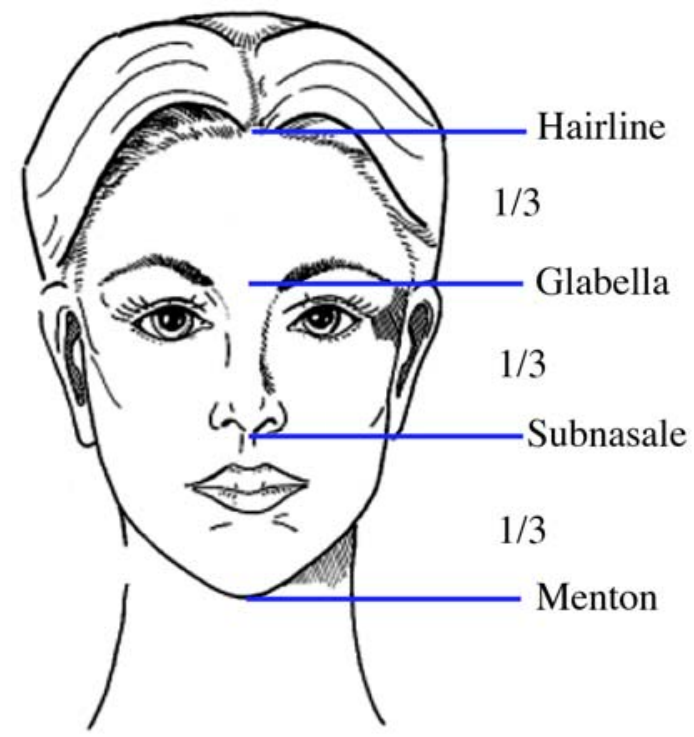

Figure 4. An example of facial trisection, as originally described by Vitruvius (ca. 70-ca. 25).

Modified from Edler RJ.

Background considerations to facial aesthetics. J Orthod 2001;28:159-68. 
Artistic analyses of facial ideals and proportions continued throughout the Renaissance with the studies of Leonardo da Vinci (1452-1519) and Albrecht Dürer (1471-1528). Leonardo, described as a great artist and thinker of the Renaissance, depicted Vitruvius's theories on the proportional ideals of man in his drawing of the Vitruvian Man, often called the Canon of Proportions or the Proportions of Man (Figure 5). In this artistic example of man, the proportionate human form is presented within a circle and square based on the idealistic guidelines of Vitruvius. Albrecht Dürer, another great painter and art theorist of the Renaissance, also believed in the importance of studying facial proportions and wrote Vier Bücher von menschlicher Proportions, a work consisting of four books published in 1528 that incorporated esthetics, art and the science of human anatomical proportions. From his research, Dürer developed a coordinate system of the face. Figure 6 is an example of Dürer's coordinate system showing differences between facial types of retroclined and proclined contours. Leonardo (Figure 7a) and Dürer (Figure 7b) both evaluated sagittal and vertical relationships of the face and purposed ideal proportions of esthethically pleasing profiles.

Other well known artistic examples of esthethic ideals include Leochares's Apollo Belvedere (Figure 8) (Angle 1900), the Venus de Milo (Figure 9) (Angle 1900), Donatello's Saint George (Figure 10), and Michelangelo's David (Figure 11) (Baum 1966).

\section{Anthropometric Guidelines}

Anthropometrics, the measurement of man, has been a valuable resource in establishing esthetic guidelines of the face. Through anthropometrics and craniometrics, anatomist Petrous Camper (1722-1789) was able to identify craniofacial differences, develop facial analyses, and lay the foundation for esthetic facial assessments.

Petrous Camper, a comparative anatomist, physician and painter, employed angles in measuring the craniofacial profile. From his scientific measurements of the human skull, Camper simplied the craniofacial profile into a geometric expression proposed as the "facial angle," which was formed by the intersection of a line drawn from the base of the nose to the base of the skull and the linea facialis, a line from the nose to the most prominent point on the forehead (Figure 12). His linea facialis became a universal anthropometric measurement used for the study of the human face and racial differentiation of craniofacial structures (Tremouth 2003). 


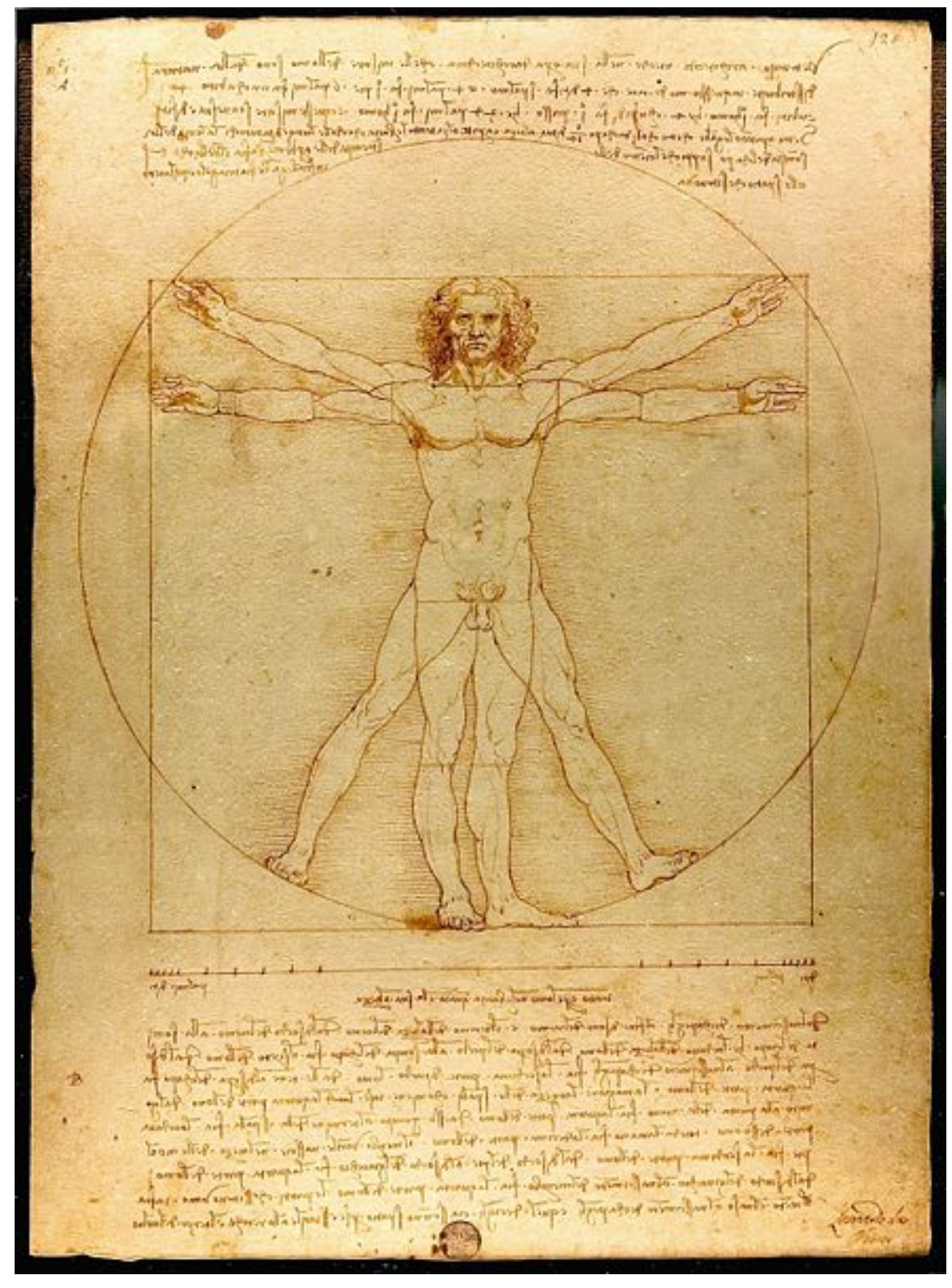

Figure 5. Leonardo da Vinci's Vitruvian Man (ca. 1485), also known as the Canon of Proportions or the Proportions of Man.

Reprinted by permission from Luc Viatour. http://en.wikipedia.org/wiki/Image:Da_Vinci_Vitruve_Lu c_Viator.jpg. Accessed January 24, 2008. 


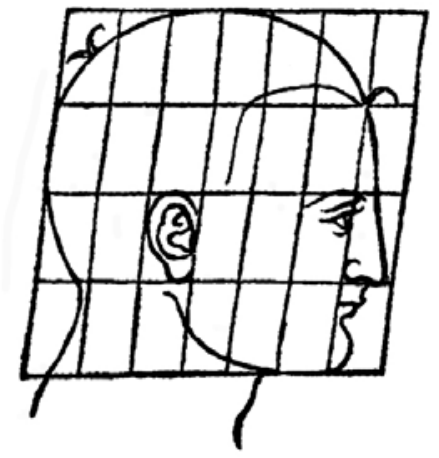

(a)

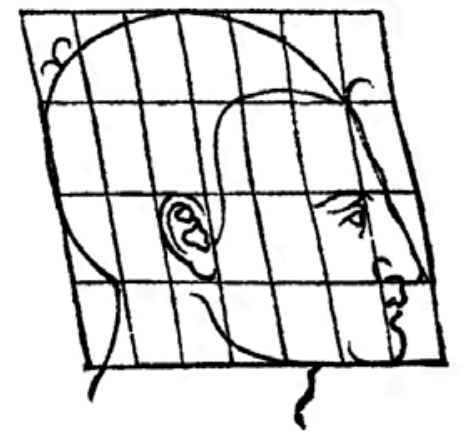

(b)

Figure 6. Albrecht Dürer's depiction of (a) retroclined and (b) proclined facial contours from the angle formed between the vertical and horizontal axes of his coordinate system.

Modified from Wahl N. Orthodontics in 3 millennia. Chapter 7: Facial analysis before the advent of the cephalometer. Am J Orthod Dentofacial Orthop 2006;129:293-8. 


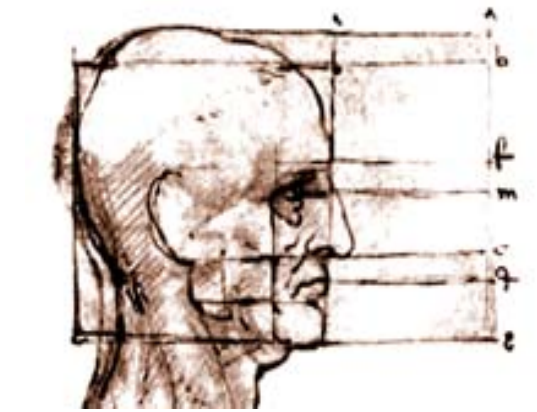

(a)

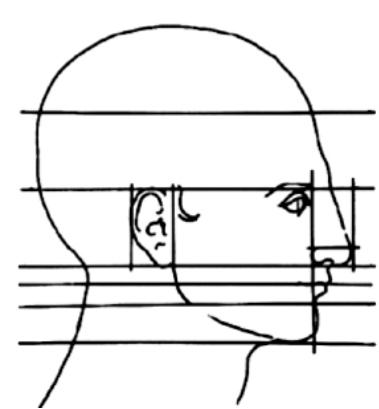

(b)

Figure 7. Vertical and sagittal relationships of the facial profile by Leonardo (a) and Dürer (b).

Modified from Edler RJ. Background considerations to facial aesthetics. J Orthod 2001;28:159-68. 


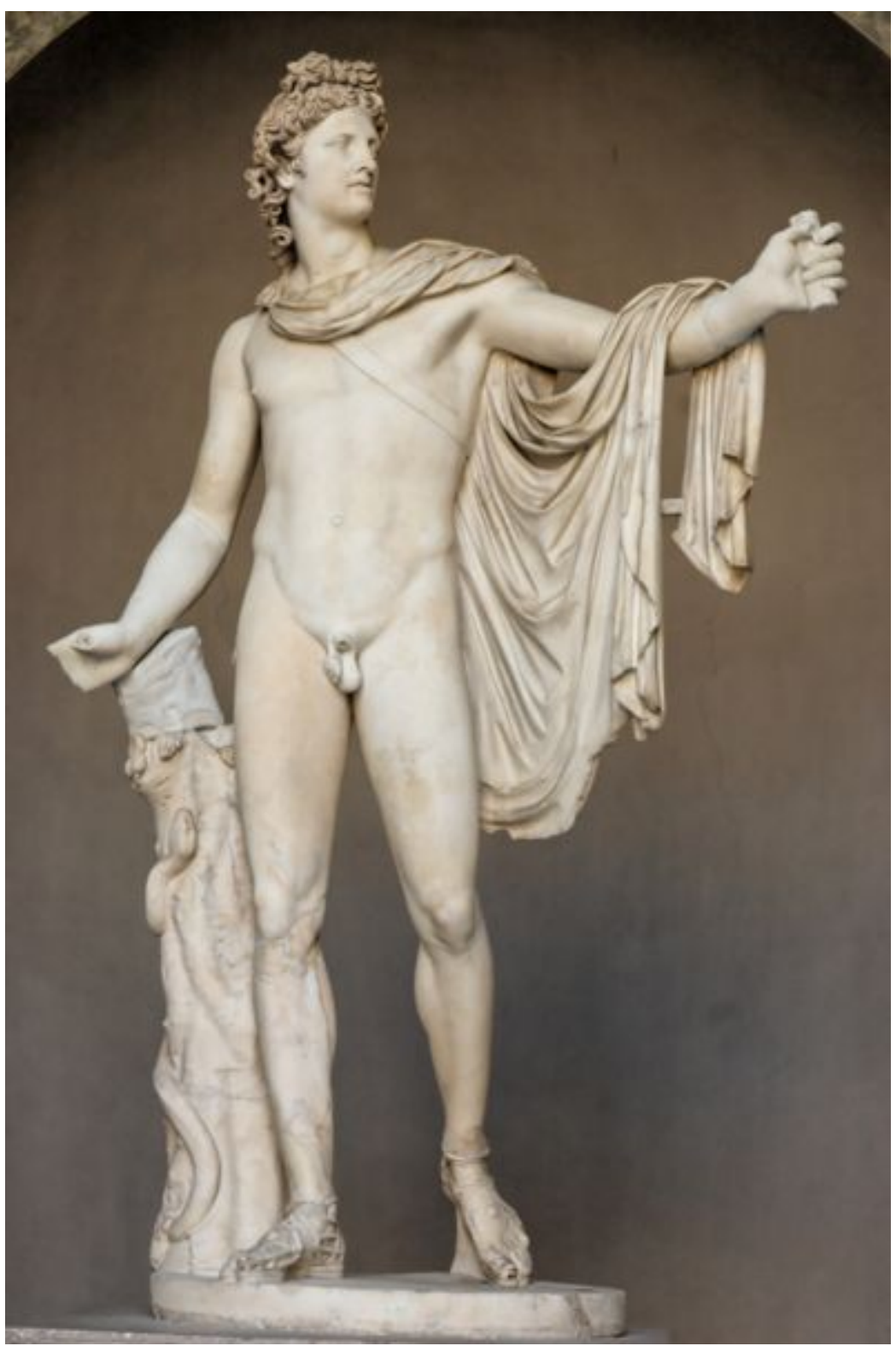

Figure 8. A Roman copy of Apollo Belvedere, representing one of the greatest legacies of Greek art, originally thought to have been produced in bronze by the Athenian sculptor Leochares between 350 to 325 B.C.

Reprinted by permission from Wikimedia Commons. http://en.wikipedia.org/w/index.php?title=Image:Belvedere Apollo_Pio-Clementino_Inv1015.jpg\&printable=yes. Accessed August 29, 2007. 


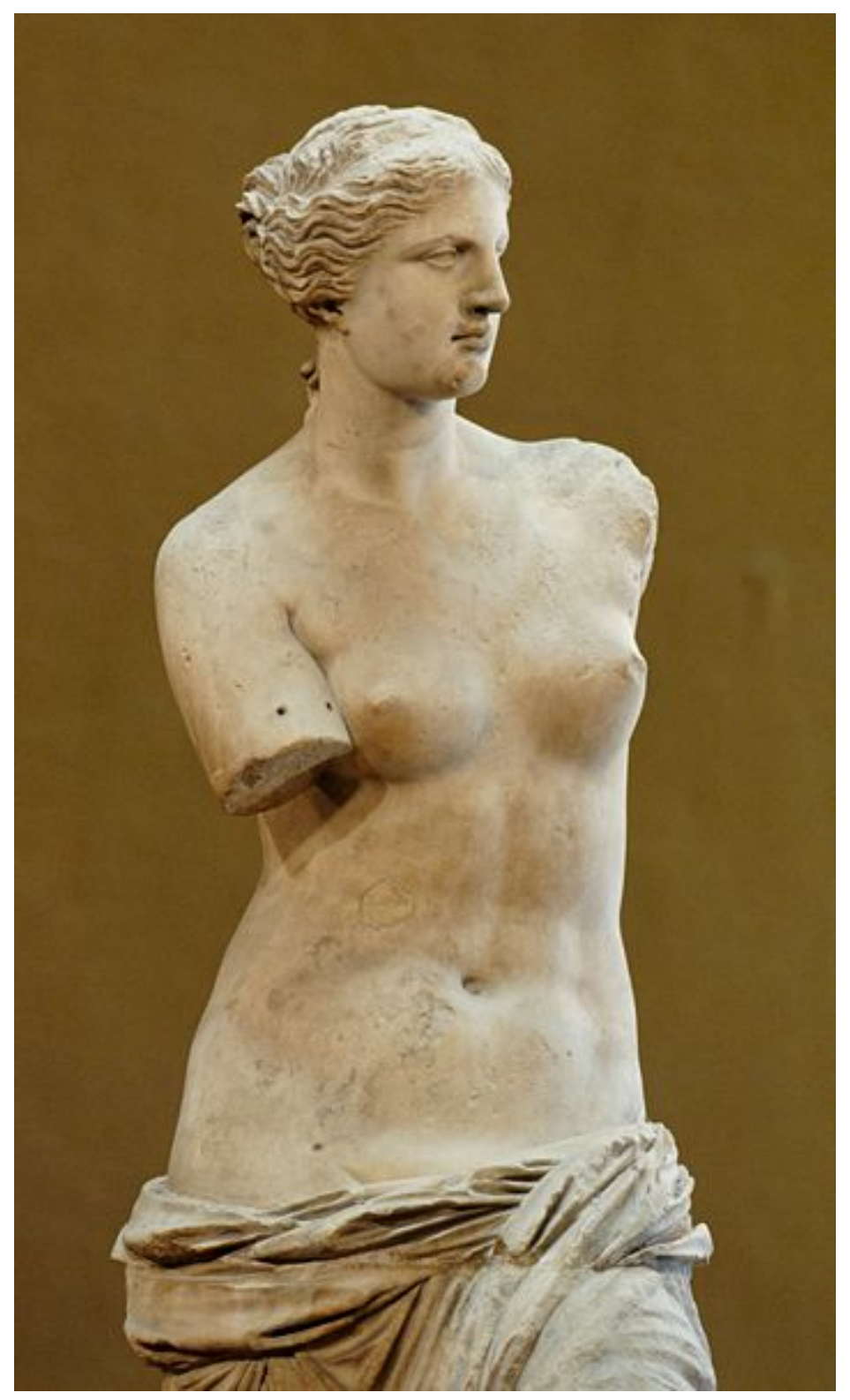

Figure 9. The Aphrodite of Milo, better known as Venus de Milo, an ancient Greek statue presently on display at the Louvre Museum in Paris dates back to 130-90 B.C.

Reprinted by permission from Wikimedia Commons. http://en.wikipedia.org/wiki/Image:Venus_de_Milo_L ouvre_Ma399_n4.jpg. Accessed August 29, 2007. 


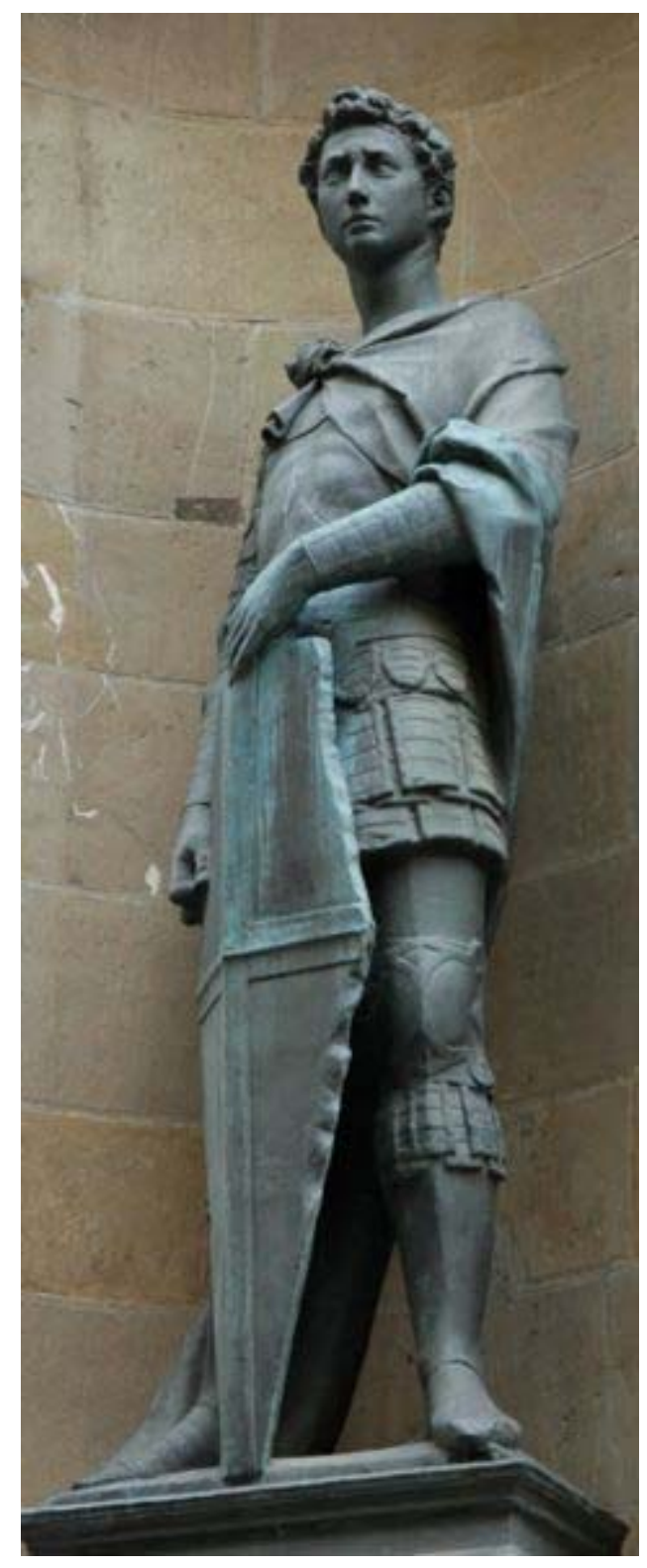

Figure 10. Donatello's Saint George (ca. 1415-1417).

Reprinted by permission from Wikimedia Commons.

http://en.wikipedia.org/w/index.ph p?title=Image:St_George_Donatello_O rsanmichele_n1.jpg\&printable=yes. Accessed August 29, 2007. 


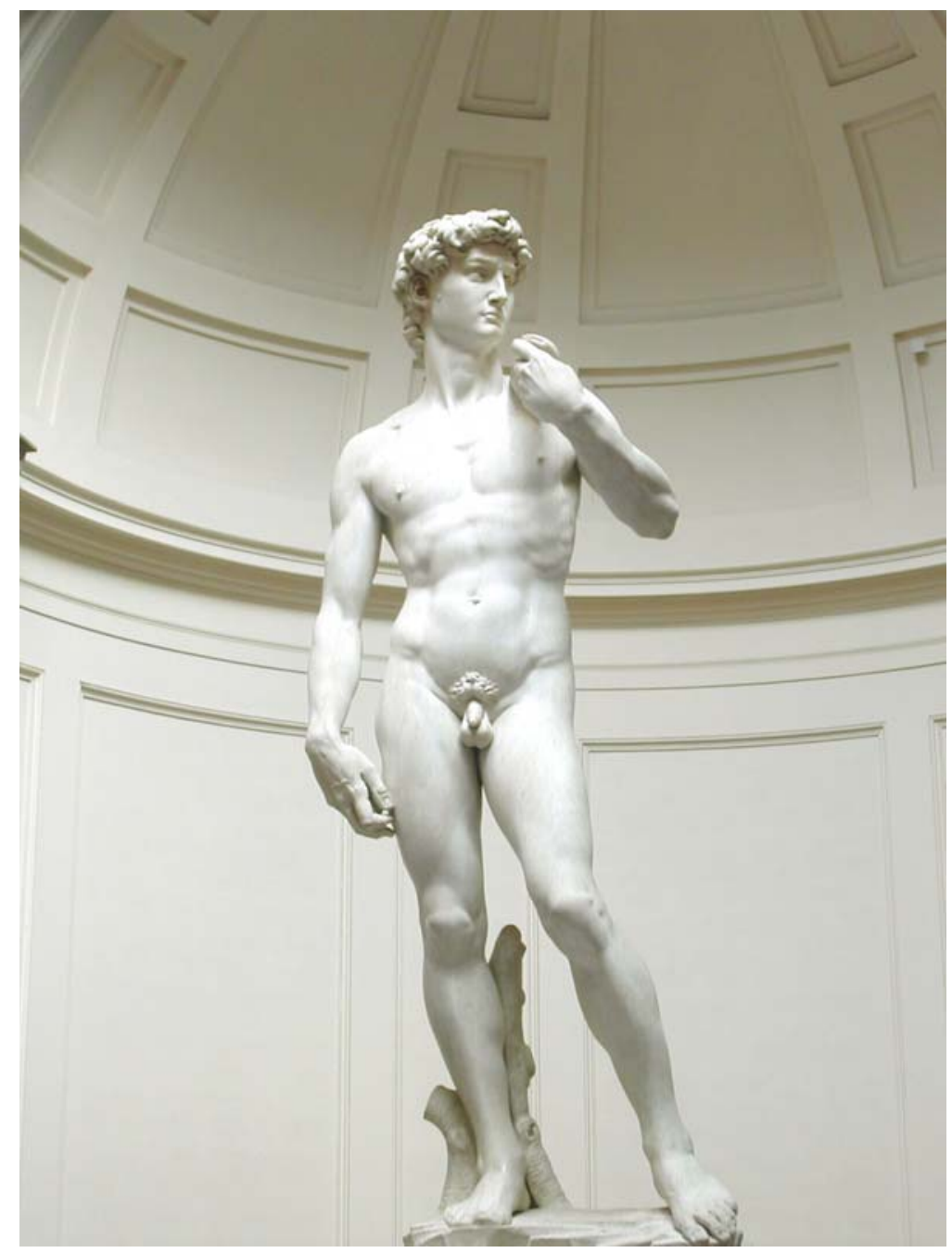

Figure 11. Michelangelo's David (ca. 1501-1504) is a 17 foot marble statue portraying the Biblical King David preparing for battle against Goliath.

Reprinted by permission from Wikimedia Commons. http:/ / en.wikipedia.org/w/index.php?title=Image:Michela ngelos_David.jpg\&printable=yes. Accessed 29, 2007. 


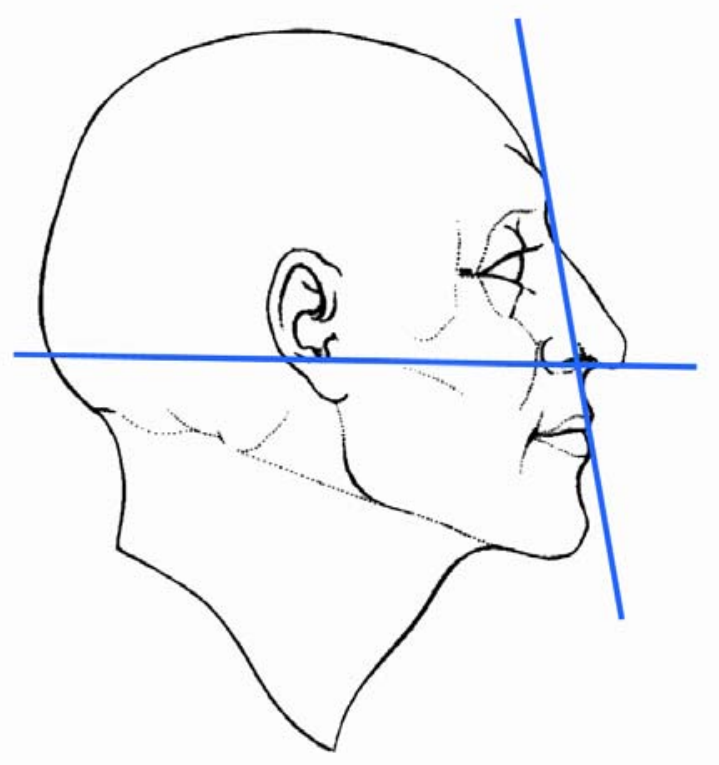

Figure 12. Illustration of Camper's "facial angle," that was based on comparative anatomy. 


\section{Cephalometric Guidelines}

Cephalometric guidelines for facial esthetics were first introduced after the development and standardization of the roentgenographic cephalometer by B. Holly Broadbent, Sr., in 1931. This made it possible to assess relationships of teeth to craniofacial structures and allowed clinicians to evaluate facial esthetics and diagnose facial disproportions more objectively.

Many cephalometric guidelines and analyses have been developed to help measure the integumental profile including Burstone's contour and inclination angular measurements (Burstone 1958), Ricketts's esthetic plane (Ricketts 1960), Merrifield's profile line and Z angle (Merrifield 1966) and Holdaway's soft-tissue cephalometric analysis (Holdaway 1983, 1984). Most of these analyses of the integumental profile, however, have been personally derived, often with unclear population details or diminutive population sizes.

In 1958 Burstone proposed a method to analyze the integumental profile from angular cephalometric measurements, namely contour angles (Figure 13) and inclination angles (Figure 14). His sample included 40 Caucasians (15 male, 25 female), with a mean age of 23.8 years, chosen to have acceptable faces by three artists at the Herron Institute of Art, Indianapolis. From this he proposed an integumental profile grid using the average scores from his sample as a wiggle plot defining a desirable and esthetically pleasing profile to be used as a goal in orthodontic treatment (Burstone 1958).

The esthetic plane (Figure 15), also called the E-plane, was developed by Ricketts (1960) to relate the lips to the soft tissues of the nose and chin. The esthetic plane is drawn from the tip of the nose to the tangent of the soft tissue chin. Ricketts suggested that in Caucasian adults, the lips should be contained within ( $4 \mathrm{~mm}$ posterior, $\pm 3 \mathrm{~mm}$ ) the esthetic plane to have facial harmony (Ricketts 1960). He, however, came to this conclusion from a sample of 1,000 subjects, primarily all Caucasian, he personally felt possessed an esthetically pleasing profile.

The profile line (Figure 16), named by Merrifield in 1966, is a reference line used to analyze the integumental profile. This line is drawn from the most anterior point of the most protrusive lip to a line tangent to the soft tissue chin. Merrifield's $Z$ angle (Figure 16) is measured from the angle formed by the profile line and Frankfort horizontal (Figure 17) enabling the clinician to evaluate profile esthetics with the ideal range being $72^{\circ}$ to $78^{\circ}$ (Merrifield 1966). Again, this ideal was derived from a sample, made predominately of American white females, he personally believed to demonstrate "outstanding" harmony and balance of the integumental profile (Merrifield 1966, p. 813). 

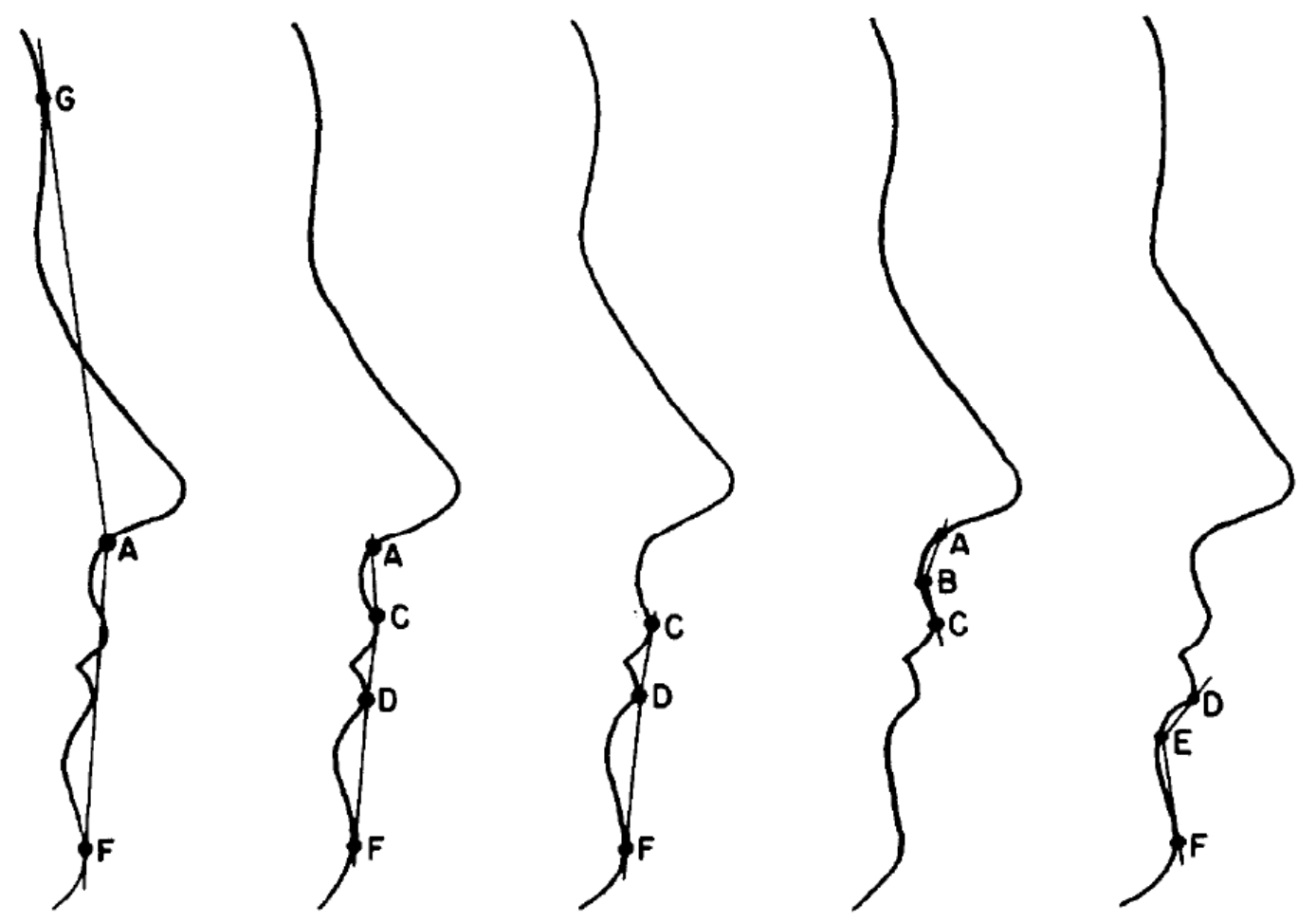

Figure 13. Representation of Burstone's five contour angles.

Reprinted by permission from Elsevier Limited.

Burstone CJ. The integumental profile. Am J Orthod 1958;44:1-25. 


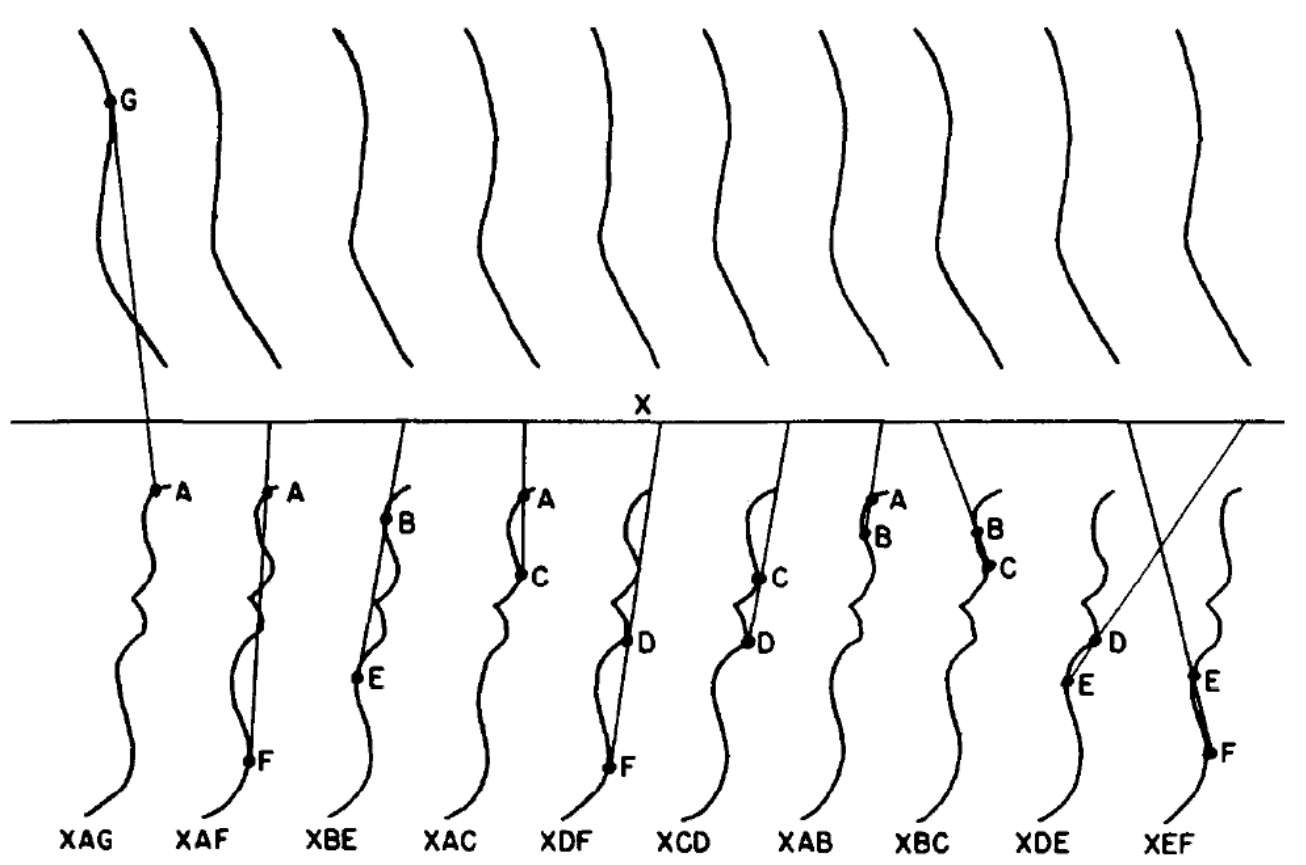

Figure 14. Representation of Burstone's 10 inclination angles.

Reprinted by permission from Elsevier Limited. Burstone CJ. The integumental profile. Am J Orthod 1958;44:1-25. 


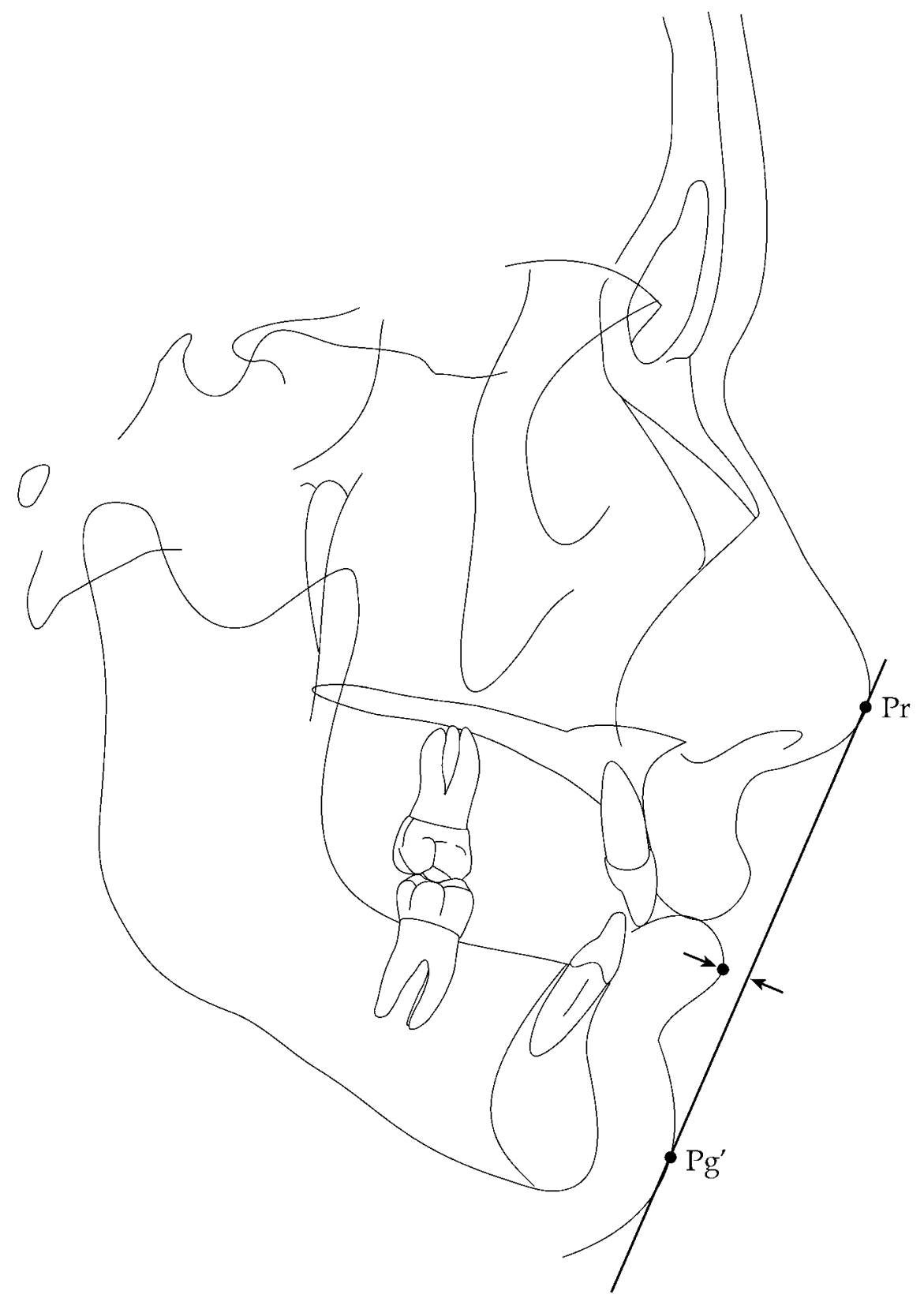

Figure 15. Schematic tracing of a lateral cephalogram showing construction of Ricketts's esthetic plane. This is drawn through the tip of the nose (Pronasale) and the tangent of the soft tissue chin $\left(\mathrm{Pg}^{\prime}\right)$. 


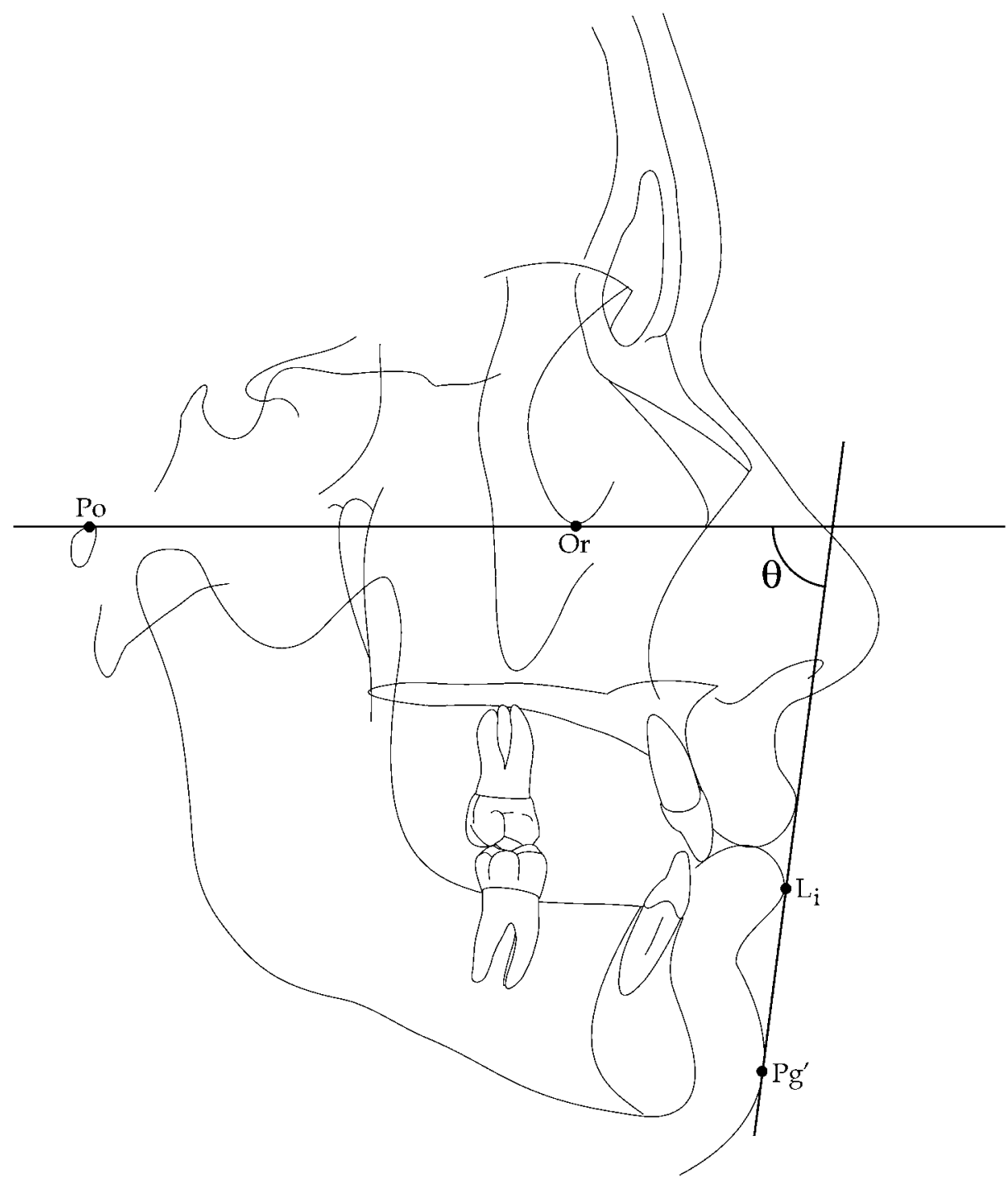

Figure 16. Merrifield's Z angle, the inferior-posterior angle between Frankfort Horizontal (Porion and Orbitale) and Merrifield's profile line (a line drawn through the most prominent lip and the tangent of the soft tissue chin). In this diagram, both lips are equally prominent (and thus coincident along the line), and the diagram is drawn to Labrale inferius. 


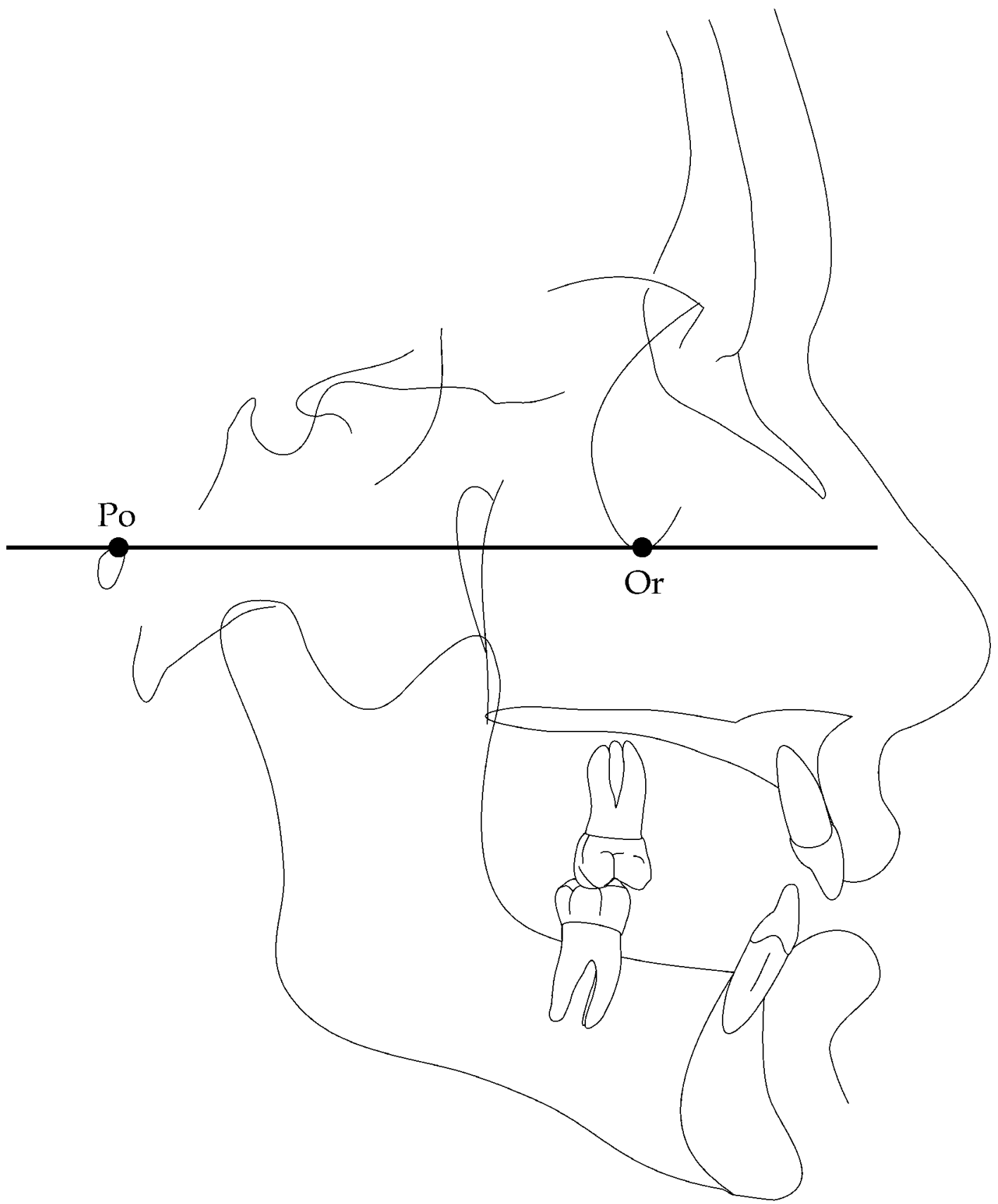

Figure 17. Schematic tracing of a lateral cephalogram showing construction of Frankfort Horizontal. This is the reference line drawn through Orbitale and Porion. 
Holdaway is accredited with developing a soft-tissue cephalometric analysis, from an unknown sample, to aid in treatment planning. This analysis is defined by seven points associated with facial beauty and harmony; they are as followed:

1. A soft tissue chin nicely positioned in the facial profile.

2. No serious skeletal profile convexity problems.

3. An $\mathrm{H}$ angle [the angle formed from soft tissue nasion to soft tissue pogonion and Holdaway's $\mathrm{H}$ line, the tangent drawn from the tip of the chin to the upper lip] that is within $1^{\circ}$ or $2^{\circ}$ of average for the convexity measurement of the individual. This angle varies according to the specific ANB angle for each patient, but a patient with an ANB angle of 3 degrees should have an $\mathrm{H}$ angle of $7^{\circ}$ to $9^{\circ}$ for an esthetically pleasing profile (Holdaway 1956).

4. A definite curl or form of the upper lip, measuring within the narrow range of $4 \mathrm{~mm}$ to $6 \mathrm{~mm}$ in depth of the superior sulcus to the $\mathrm{H}$ line and within 2.5 $\mathrm{mm}$ to $4 \mathrm{~mm}$ from a perpendicular line drawn from Frankfort Horizontal.

5. The lower lip either on the $\mathrm{H}$ line or within $1 \mathrm{~mm}$ from it.

6. Lower lip form and sulcus depth harmonious with those of the upper lip, although more variation is seen in this area than in the upper lip.

7. No unusually large or small measurements of either total nose prominence or soft tissue chin thickness (Holdaway 1983).

Holdaway's seven points illustrate how the soft tissue profile could vary and still be considered esthetically pleasing (Holdaway 1984). Again, these were Holdaway's conclusions after years of observation in private practice, from an unknown sample population and size.

\section{Philosophical Debate}

Cognition of beauty, as defined from the online encyclopedia, Wikipedia, "involves the interpretation of objects as being in balance and harmony with nature, which in turn elicits a sense and experience of attraction, affection, and pleasure." The philosophical question regarding beauty and facial attractiveness is whether one's perception of beauty is subjective or not.

Is facial beauty simply a characteristic, or is it dependent on one's own ideas and feelings that directly affect one's sensory enjoyment? The philosopher David Hume (1741) felt beauty was subjective and stated "beauty in things exists in the mind which contemplates them." This too was believed by Margaret Wolfe Hungerford (1878), whose well known quote "beauty is in the eye of the beholder," assumes beauty to be a subjective perceptual sense within each person. Contrary to this perspective, two 18th century philosophers, Francis 
Hutcheson and Immanuel Kant, argued that beauty was a quality common to all. Hutcheson said, "esthetic judgments are perceptual and take their authority from a sense that is common to all who make them," and "the origin of our perceptions of beauty and harmony is justly called a sense because it involves no intellectual element, no reflection on principles and causes" (Hutcheson 2004). Kant (1790) supported Hutcheson's philosophical ideologies by saying that, "beautiful is that which pleases universally without a concept." This raises the question, is it possible to make judgments about the quality of facial appearances? Can an agreement of improvements in facial attractiveness be considered cogent?

Psychological literature suggests that our perceptions of beauty are both inherent and universal, meaning they are in fact genetic in origin and crosscultural. Although it has been shown that amongst children, ages 3 to 4 , the perception of attractiveness of their peers was environmentally induced (e.g., Berscheid and Walster 1974; Dion and Berscheid 1974; Langlois 1986), there is abundant evidence within the psychological literature to suggest that perceptions of facial beauty are founded in our heredity. Some psychological studies have indicated that an infant as young as 3 months of age can distinguish between attractive and unattractive faces (Samuels and Ewy 1985; Langlois et al. 1987). Langlois et al. (1987) showed that when infants 3 and 6 months of age were shown previously assessed faces judged as attractive or unattractive, they showed distinct signs of preference for the attractive faces. Langlois et al. therefore concluded that since it was highly unlikely that by the third month of age an infant could or would have respond to any cultural or environmental influences, the perception of attractiveness was inherent or genetic in origin.

A number of psychological studies have concluded that perception of attractiveness is also universal. In a cross-cultural comparison Thakera and Iwawaki (1979) showed that Asian, English, and Oriental female raters showed similar agreement in interpersonal attractions of a selection of ancient Greek males. Another cross-cultural comparison by Bernstein et al. (1982) showed a strong agreement in attractiveness ratings when comparing Chinese and Caucasian attitudes. In addition, reciprocal studies completed by Maret (1983) and Maret and Harling (1985) evaluated cross-cultural perceptions of attractiveness between a combined group of male and female raters of either Cruzan (native of US Virgin Islands) or American White racial origin. In the first study Maret (1983) found that both the Cruzan and the American White groups similarly assessed a group of Cruzan subjects according to facial attractiveness. Maret and Harling (1985) then proved that from similar constituted groups of Cruzan or American White raters both agreed on the attractiveness of American Whites. These and other studies from the psychological literature support the conclusion that collective judgments can be made about the quality of facial 
appearance and attractiveness since its perception is a universal phenomena with presumably a common evolutionary biological basis.

The philosophical debate of beauty has not been overlooked within the orthodontic community. Richard S. Hambleton's article, “The Orthodontic Curtain," which was read before the Southern California Component of the Angle Society in 1962, recognized that the ideal face varies among individuals (Hambleton 1963). Daniel J. Subtelny also states that the face each individual deemed pleasing is not the same, stating it exists only in the "mind's eye," of the individual (Subtelny 1961). Much earlier, Wuerpel had expressed to his friend Angle this same belief, that beauty was dependent upon the spectator and that no single facial form could be taught as esthetic perfection (Wahl 2006). Morris M. Stoner (1955) suggested each individual's concept of facial beauty and esthetics was a function of his or her own "innermost sensibility and understanding." But with this said, Stoner fully contended that there is substantial agreement among many that certain faces fall within the definition of "harmony in form," and it is from this which the orthodontic community has developed its standards (Stoner 1955).

\section{Esthetic Ideals in Orthodontics}

Esthetic ideals used in orthodontic diagnosis and treatment planning have evolved from artistic, anthropometric and cephalometric guidelines. The effect orthodontic treatment can have on facial esthetics has directed the progression of the orthodontic specialty for the past century. Two influential figures in orthodontics, Calvin S. Case and Edward H. Angle, established that facial attractiveness and balance did not depend solely on aligned teeth but in the sum total of interactions between all structures that comprise the dentofacial complex.

The first to argue for the importance of the facial profile in determining optimal orthodontic treatment was Calvin S. Case (1847-1923). Case stated that, "in the correction of all malocclusions the facial outlines should be regarded as an important guide in determining proper treatment" (Case 1922). Unfortunately Cases's contentions were overshadowed by his professional rival, Edward H. Angle.

Edward H. Angle (1855-1930), credited with making orthodontics a dental specialty, originally emphasized the highest aim in orthodontics to be the "restoration" of normal occlusion, without concern for effects on facial attractiveness. In search for more definitive esthetic goals in orthodontics, Angle employed the help of his close friend, Edmund H. Wuerpel (1856-1958). Wuerpel was an artist and art teacher at Washington University who spoke 
before the Edward H. Angle Society of Orthodontia on multiple occasions and published articles concerning facial esthetics in the orthodontic literature (e.g., Wuerpel 1931, 1937). Wuerpel (1937) felt no single rule for an ideal facial form could be implemented and believed the most important factors in facial esthetics were harmony and balance with no single element of the profile overemphasized at the expense of another. The balanced face is described by B. L. Herzberg (1952) as a face in which "the chin does not protrude or recede; the lips, either upper or lower, are not in protrusion or obviously thick; the mental depression is not deep with a rolling forward lower lip and there is no tension in the triangularis muscle region" (Herzberg 1952, p. 4). Wuerpel (1937) applauded Angle's ability to restore normal occlusions in his patients, but questioned at what cost to facial esthetics was it achieved. Angle responded by saying, "how in thunder was I to know that I should have considered [facial esthetics]? I thought the restoration of the normal position of the teeth was my problem and that nothing else mattered" (Wuerpel 1937, p. 82). With this realization Angle taught that the profile of Apollo Belvedere (Figure 8) was a desirable goal of orthodontic treatment (Angle 1907). However, he distinctly felt it had limited uses in gauging harmony, or disharmony, of faces. Angle felt it more important, not whether the face conformed to a given standard, but whether the features of the individual were in harmony (Angle 1900).

Their claims have since been investigated in hopes of instituting treatment options that improve the facial attractiveness of patients (e.g., Burstone 1958; Ricketts 1960; Merrifield 1966; Holdaway 1983, 1984). Clinicians within the specialty have studied and examined (1) what constitutes an esthetic facial profile and (2) what effects orthodontic treatment can have on the integumental profile. These two concerns still lack definitive answers.

\section{Significance in Orthodontics}

The integumental profile is intimately related to the skeletal framework of the face. Changes seen in either the skeletal frame or the soft tissue covering of the face will inherently affect the profile. These changes can occur in relation to growth, but they can also be accredited to orthodontic therapy. Soft tissue changes incident to growth encompass a greater aspect of the soft tissue profile, including the nose and chin, as well as the lips (Subtelny 1961; Hambleton 1964; Vig and Cohen 1979); whereas soft tissue changes incident to orthodontic treatment will center on the lips (King 1960; Hambleton 1964; Rundee 1964). The effects of orthodontic therapy on facial esthetics has been a long-term concern within the orthodontic community (Angle 1900; Wuerpel 1937; Hertzburg 1952; Burstone 1958; King 1960; Subtelny 1961; Hambleton 1963; Spyropoulos and Halazonetis 2001), but these effects also are a concern of 
orthodontic patients and their parents (Shaw et al. 1979; Shaw 1981; Shaw et al. 1985; Vig et al. 1999; Birkeland et al. 2000). Since people seeking orthodontic treatment are often seeking an improvement in facial harmony, Charles J. Burstone stated, "Modern orthodontics implies not only occlusal excellence, but also the positioning of teeth to produce optimal facial harmony for the individual patient" (Burstone 1958, p. 24). He further stated that orthodontic treatment can produce both desirable and undesirable alterations in the integumental profile (Burstone 1958).

Other investigators, however, contend that alveolar bony changes due to orthodontic therapy may or may not influence the integumental profile (Salzmann 1964; Hershey 1972; Wisth 1974). It has been contended that a reduction in lip prominence is not always related to tooth movement, but is instead largely dependent upon muscle size and tonicity, muscular habits, and psychosomatic involvement (Salzmann 1964). Others have reported that the soft tissue response from incisor retraction was unpredictable (Hershey 1972; Wisth 1974).

\section{Previous Esthetic Evaluations in Orthodontics}

Many subjective esthetic evaluations of facial profiles have been published using different presentations (e.g., line drawings, photographs, and silhouettes), several observer panel compositions (e.g., lay persons, dental professionals, and dental specialists) of various sizes, and various measuring techniques (e.g., ordinal scales and visual analogue scales). After a review of subjective esthetic evaluations, two previous esthetic evaluations examining profile attractiveness following functional therapy are discussed here.

\section{Line Drawing Evaluations}

Lines et al. (1978) verified significant differences in profile preferences for males and females using line drawings of profiles. A ranking scale was used by panels of medical and dental students, oral surgeons, orthodontists, dental hygienists, dentists, and lay people to find the facial profile components considered desirable for males and females from these line drawings. Line drawings were again used by Prahl-Anderson et al. (1979) to confirm a difference between professionals' and lay persons' ratings of the profile. Observers used a 3-point predetermined rating scale, with semantic phrases (1-normal, 2-slightly deviating, but not disturbing, and 3-abnormal, requiring treatment). Later, Bowman and Johnston (2000) used line drawings of profiles from cephalometric tracings to conclude that extraction treatment improved facial esthetics in Caucasian subjects who had protrusion and crowding before treatment. This 
was confirmed by a panel of dentists and lay persons asked to determine which profile they preferred from randomly ordered profile line drawings (pretreatment on the left and posttreatment on the right, and vice versa). They then were asked to mark their preference using a VAS anchored by the terms the same and very much better. The scores from each of the two panels were averaged for each subject to produce a single dentists' score and a single lay persons' score.

\section{Photographic Evaluations}

Photographic images have also been used for esthetic evaluations of the profile. Dongieux and Sassouni (1980) evaluated the esthetics of subjects with varying anteroposterior and vertical positions of the mandible. They used neutralized black and white frontal and profile photographs to counteract extrinsic factors (e.g., makeup, hair style, and complexion) and concluded that the integumental profile was more dependable than the frontal view in assessing the esthetics of subjects with different mandibular positions. A 5-point numeric scale with the following semantic phrases: (1) very pleasing, (2) pleasing, (3) average, (4) unpleasing, (5) very unpleasing was used by panels of orthodontists, lay people, and artists.

Lundström et al. (1987) also used frontal and profile photographs to examine the esthetics of untreated patients with vertical or horizontal growth patterns evaluated by a panel of orthodontists, orthodontic residents, artists, and lay people. In this study a new 5-point numeric and semantic scale ranging from 1 (very good-looking) to 5 (very disharmonious) was used to confirm that subjects with a vertical growth pattern were more disharmonious than subjects with a horizontal pattern of facial growth. Similarly, to score the amount of change from age 12 to 18 the following scale was used: $0=$ no change, $+1=$ improvement of one unit (e.g., from average to good-looking), $-1=$ deterioration of one unit (e.g., from disharmonious to very disharmonious). Each panel reevaluated the cases one week after their initial assessment to test the consistency between group means, determined from correlation coefficients (ranging between 0.75 and 0.92; $\mathrm{P}<0.01$ ) showing good agreement between the two assessments by each panel. Consistency was also tested of the means and standard deviations of the mean assessments of each panel for male and female subjects to suggest good reliability of mean assessments calculated using Student's $t$-test. Student's $t$-test was also used for judging statistical significance of mean differences between groups with the exception of lay people, which found cases to be better-looking, and artists, which were more critical. To determine whether the changes in the assessments of the same subject between the ages of 12 and 18 were assessed reliably, a cross-correlation between panel groups based on mean changes scored from the original evaluation was done to show all were either highly significant $(P<0.01)$ or significant $(P<0.05)$ for male 
subjects, but one panel (the senior orthodontic residents) showed less ability in detecting changes in females when compared to the other panel groups.

Kerr and O'Donell (1990) had groups of four orthodontists, dental students, art students, and parents of children undergoing orthodontic treatment evaluate frontal and lateral esthetics before and after orthodontic treatment from photographs of subjects with different malocclusions. Using the same 5-point numeric scale used by Lundström et al. $(1987,1989)$, they found that Class I faces were rated as more attractive than Class II, division 1 or Class III faces; and that overall attractiveness of all subjects improved following treatment $(\mathrm{P}<0.001)$, with Class II, division 1 subjects having the most significant improvement $(\mathrm{P}<$ 0.001) over Class I subjects (no significant difference in ratings after treatment) and Class III subjects $(\mathrm{P}<0.05)$. Kerr and O'Donell (1990) used Duncan's Multiple Range Test to compare the mean ratings for (1) each panel, (2) each Angle classification with all panels combined, (3) frontal and lateral views of each Angle classification with all panels combined, (4) before and after treatment of each Angle classification with all panels combined, and (5) before and after treatment of each Angle classification by each panel. Since Lunström et al. (1987) showed the rating method used was reproducible, consistent, and therefore reliable no test of reproducibility was performed.

Proffit et al. (1992) compared nongrowing Class II subjects treated with orthodontics alone to subjects who underwent orthodontic treatment along with mandibular advancement surgical procedures using frontal and profile photographic slides. Orthodontic residents, maxillofacial surgery residents, orthodontists, and maxillofacial surgeons evaluated the profile esthetics of these subjects using a VAS. The rating scores were analyzed by a multivariate analysis of variance and disclosed no significant difference between the oral surgeons and orthodontists when comparing the surgical and orthodontic subjects, either before or after treatment. The results showed higher initial and final mean ratings for the subjects with orthodontic treatment alone, but greater amounts of esthetic improvements were found for the orthognathic subjects.

Cochrane et al. (1999) used black and white photographs for the profile evaluations of subjects with different types of malocclusions (Class I, Class II, and Class III). Groups of lay people, dental students, orthodontists, and maxillofacial surgeons favored Class I profiles most often and rated Class II profiles to be the least attractive.

Kiekens et al. (2005) used three sets of photographs (a frontal, a profile, and a three-quarter smiling) to evaluate the reproducibility and validity of a ratio scale they created to judge facial esthetics. Their ratio scale incorporated a set of previously scored reference photographs (one for males and one for females) and 
a VAS that was marked with the reference photographs' score. This allowed the observers, who included adult lay persons, orthodontists, and post-graduates, to score the esthetic attractiveness of the subjects that presented with different malocclusions as a ratio compared to the reference set's score. Comparing the reproducibility of this new scale, the reliability coefficient for the final overall score of each subject both for the lay people and the professionals was excellent, calculated by Cronbach's alpha ( $\geq 0.98)$. In testing the validity of their scale, the photographic three-quarter smiling views $(n=44)$ were also evaluated by a second panel of orthodontists and orthodontic residents using a previously published scale for three-quarter smiling photos labeled the Peerlings scale (Peerlings et al. 1995). Statistically significant correlations were found by calculating the Pearson's correlation coefficients between the mean scores of the three-quarter smiling photos from the new and previously published scales for both the lay persons $(n=42 ; r=0.82 ; P<0.05)$, and the professionals $(n=42 ; r=$ 0.77 ; $\mathrm{P}<0.05)$ which validated their new scale, meaning that it measures what it claims to. Therefore, no logical errors occur when formulating conclusions from its data. After showing that this ratio scale was reproducible, capable of giving consistent results, and valid, an efficacious measuring technique, the only statistically significant esthetic conclusion drawn from this study was that Class II, division 2 subjects were more attractive than Class III subjects.

\section{Silhouette Evaluations}

In recent years, silhouettes have become more commonly used for esthetic evaluations of the profile. Silhouettes have been criticized for their simplification of facial esthetics, eliminating extrinsic (e.g., hair style, make-up) and intrinsic (e.g., skin complexion, hair color) factors. They are described as only a complement to other methods of esthetic evaluation; however, they are suitable for the comparison of change within the same profile (Barrer and Ghafari 1985). Many others (e.g., De Laat 1974; O'Neill et al. 2000; Shelly et al. 2000; Spyropoulos and Halazonetis 2001; Mergen et al. 2004) have supported the use of silhouettes due to their simplicity and disregard of variable factors that have been shown to bias the ratings of profile esthetics (Spyropoulos and Halazonetis 2001). The following is a chronological review of studies that used silhouettes to evaluate profile attractiveness within the orthodontic literature.

For his Masters thesis, De Laat (1974) used silhouettes when comparing lay people's perceptions of pretreatment and posttreatment profiles of subjects initially presenting with Class I and Class II, division 1 malocclusions treated with premolar extractions. He found that most lay persons saw an improvement in facial harmony posttreatment, especially in the Class I subjects. 
Barrer and Ghafari (1985) asked first-year dental students to choose the profile they preferred from silhouettes of Class II, division 1 subjects before and after treatment with either (1) a Fränkel appliance and the Begg light-wire technique, (2) the straight-wire method, or (3) Tweed Edgewise mechanics. The dental student then classified his or her selection as being satisfactory or unsatisfactory. The results showed that posttreatment profiles were more commonly preferred by all groups, with satisfactory posttreatment ratings ranging from $46 \%$ to $55 \%$ and no clear trend in preference among treatment modalities.

In a randomized control study, O'Neil et al. (2000) used silhouettes when evaluating Class II, division 1 profiles of untreated subjects and subjects treated with either a Fränkel functional regulator or a Harvold activator. After 18 months, changes in profile attractiveness were assessed by panels of dental students, art students, and parents of orthodontic patients composed of equal numbers of male and female observers. After first deciding which profile they preferred, the initial or 18-month profile, the observers used a ratio analogue scale (a VAS anchored by the descriptors $0 \%$ and $100 \%$ more attractive with reference examples of a very unattractive, average, and very attractive silhouette image, which in this study was selected by only two orthodontists) to score how much more attractive they felt it to be. No statistical significant difference was found between the panels, between the male and female observers, or between the amount of change in profile attractiveness of the treated and untreated subjects. The authors concluded from this randomized control trial that, when compared to no treatment, treatment with functional appliances did not lead to more attractive profiles. A commentary written by Giddon (2000) concerning this study suggested the conclusion of no differences in attractiveness resulting from treatment with functional appliances might have been premature; it was also suggested the differences were not found statistically significant due to the extremely high variances within the respondent panels and that the individual raw scores should have been corrected by "correcting individual raw scores to $[\mathrm{z}$ scores] to help minimize the effects of the large interindividual differences" (Giddon 2000, p. 377). The use of non-gender specific reference examples of a very unattractive, an average, and a very attractive silhouette was also questioned since they were chosen by two orthodontists and were used as examples for both male and female subjects. In a letter to the editor of the American Journal of Orthodontics and Dentofacial Orthopedics, Courtney and Leigh (2001, p. 18A) responded in support of the study and its use of "randomized controls to minimize confounding variables and eliminate many of the biases that can lead to false results in nonrandomized trials." This evidence-based defense, however, relates to the debate over the validity of randomized control trials for orthodontic studies. The problem with using randomized control trials in certain clinical aspects, as in orthodontics, is that they forego the diagnostic 
process. In this randomized control trial, subjects presenting with Class II, division 1 malocclusions were matched only by age and sex and were randomly assigned to a group without any diagnostic considerations. Without proper differential diagnosis, the results of this study are likely to be misleading.

A retrospective silhouette study by Shelly et al. (2000) investigated the impact of mandibular advancement surgery (without genioplasty) on profile esthetics. These silhouettes were constructed from lateral cephalometric radiographs of mandibular deficient Class II, division 1 subjects prior to and after treatment. Two panels of 9 lay people and 9 orthodontic residents scored the esthetic of each profile using a 7-point numeric rating scale, called a Leikert scale (Isaac and Michael 1971), that ranged from 1 (less attractive) to 7 (more attractive) with no additional information. Consistently, subjects with an initial ANB angle of $6^{\circ}$ or greater were found to have an improvement in facial esthetics posttreatment, but approximately $50 \%$ of subjects with an initial ANB angle of less than $6^{\circ}$ were judged to have poorer esthetics after treatment. In their discussion, they explain that this could possibly be because all subjects underwent mandibular advancement alone and some might have benefited from a genioplasty in addition to the mandibular advancement surgery to improve esthetic ratings.

Mergen et al. (2004) also used silhouettes for the profile evaluation of Class II, division 1 subjects with varying degrees of anteroposterior and vertical dysplasias treated with comprehensive orthodontics and headgear. Groups of 9 orthodontic residents and 9 lay people evaluated pretreatment and posttreatment silhouettes created from lateral cephalometric tracings of the soft tissue profile. These silhouettes were presented using a Microsoft PowerPoint presentation (Microsoft Corporation, Redmond, Washington) with introductory examples representing the range of esthetics to be evaluated. The observers rated the esthetics of the profiles using a Leikert scale, a numeric rating scale consisting of 7-points, ranging from 1 (very unattractive) to 7 (very attractive) (Isaac and Michael 1971). It was found that as the initial skeletal discrepancies worsened, the initial profiles were judged to be statistical significantly less attractive. At posttreatment, however, there was no perceived difference in the esthetic ratings of the profiles, independent of the initial skeletal discrepancy. This implied that growing Class II, division 1 subjects (males younger than 14 and females younger than 12) can undergo significant profile improvement with appropriate and timely treatment using fixed orthodontic appliances and headgear. 


\section{Observer Panel Compositions}

Several different compositions of observers have been used for subjective esthetic evaluations within the orthodontic literature, as stated earlier. The influence that background and sex have on panel members' evaluations of facial esthetics of adolescents is still, however, unclear. Although high correlations have been reported between esthetic ratings of lay people and dental professionals (Peerlings et al. 1995; O'Neill et al. 2000; Spyropoulos and Halazonetis 2001; Kiekens et al. 2005), other investigations have shown that lay people are more critical than professionals (e.g., Tedesco et al. 1983; Phillips et al. 1992; Spyropoulos and Halazonetis 2001), while other studies found the opposite (e.g., Prahl-Andersen et al.1979; Kerr and O'Donell 1990; Bowman and Johnston 2000; Kiekens et al. 2005, 2007). The age of panel members was found to have no influence on the ratings of facial esthetics (Cross and Cross 1971; Howells and Shaw 1985); however, age and sex have been suggested to possibly confound the assessments made by other panel compositions (Spyropoulos and Halazonetis 2001; Kiekens et al. 2007). Some studies indicate that sex was not influential in observer ratings (Shaw 1981; De Smit and Dermaut 1984; Barrer and Ghafari 1985; Howells and Shaw 1985; O'Neill et al. 2000), but others suggest that female observers give higher attractiveness ratings and are less critical than males (Tedesco et al. 1983; Cochrane et al. 1999). There is also a wide range of panel sizes within the literature, with as little as two observers per panel suggested to give acceptable reliability of ratings (Howell and Shaw 1985). In an investigation on panel composition by Kiekens et al. (2007), a panel of seven randomly selected lay persons or orthodontists was found sufficient to obtain reliable results, meaning statistically repeatable, for esthetic evaluations of adolescent faces.

\section{Measuring Techniques}

Prior to the start of the present study, a quantitative method for measuring profile esthetics had to be chosen. Most esthetic evaluations found in the literature have been previously accomplished using ranking (Lines et al. 1978; Tedesco et al. 1983; De Smit and Dermaut 1984), numeric rating (Prahl-Anderson et al. 1979; Dongieux and Sassouni 1980; Evans and Shaw 1987; Lundström et al. 1987, 1989; Kerr and O'Donell 1990; Shelly et al. 2000; Mergen et al. 2004), ratio (Peerlings et al. 1995; O'Neill et al. 2000; Faure et al. 2002; Kiekens et al. 2005), or visual analogue scales (Howells and Shaw 1985; Shaw et al. 1985; Bowman and Johnston 2000; Flores-Mir et al. 2004). A ranking scale is a type of measuring scheme that asks observers to rank images in order of esthetic preference, commonly from most preferred to least preferred. A numeric rating scale is a type of predetermined numbered rating classification that is customarily described with semantic phrases, where observers are asked to classify or rate the esthetics of subjects. For example, the 5-point numeric rating scale used by 
Lundström et al. (1987) ranged from 1 (very good-looking) to 5 (very disharmonious). A ratio scale for esthetic ratings was first used by Peerlings et al. (1995) out of the Department of Orthodontics and Oral Biology at Radboud University Nijmegen Medical Centre, The Netherlands. This type of scale uses a visual analogue scale with a pre-scored reference example for observers to use to determine esthetic scores of sample subjects. Peerlings et al. (1995), Faure et al. (2002), and Kiekens et al. (2005) all used the same reference photographs (one for adolescent males and one for adolescent females) as their reference example, taken from Peerlings et al. (1995) study. In a sense, a ratio analogue scale scores subjects as a ratio in comparison to the reference example. Visual Analogue Scales (VAS) are often used as a measuring instrument for dental, dentofacial, and facial esthetics (Howells and Shaw 1985; Shaw et al. 1985; Bowman and Johnston 2000; O'Neill et al. 2000, Flores-Mir et al. 2004) by measuring feelings that are believed to range across a continuum of values that are not easily measured directly.

The use of VAS for research purposes has been used in clinical and research settings since the 1920's and is deemed most suitable in examining comparable relationships between different observations or conditions when multiple ratings are obtained from the same examiner and when measuring change within a subject or individual (Aitken 1969). A VAS is made of a straight line that is anchored on each end by right-angled stops with labels placed beyond the ends of the line naming extreme limits pertaining to the response to be measured (Huskinsson 1983), as illustrated in Figure 18. Observers respond to the scale by marking through the straight horizontal line at the point which bests describes their perception within the labeled extremes. Although vertical representations of VAS have been used, the horizontal VAS has been shown to give a greater uniform distribution of ratings (Scott and Huskissson 1976). The VAS that is most commonly used is $100 \mathrm{~mm}$ in length and is best in preventing method error (Revill et al. 1976); it is most commonly measured in millimeters from an end of the scale to the subject's mark on the line by a single observer. The use of a VAS enables ratings to be made with greater sensitivity, meaning it has a greater probability of detecting esthetic attractiveness, than with semantic phrases. Semantic phrases restrict observers to specific categories, and VAS avoid biases commonly seen with numerical rating scales (Wewers and Lowe 1990). It should, however, never be assumed that the same rating given by two examiners expresses the same level of feeling (Aitken 1969; O'Neill et al. 2000). Observers commonly ignore portions of the scale, particularly the extremes (Phillips et al. 1992). To manage this negative aspect, adjusted means can be used to compare ratings between raters. Adjusted means, or commonly called least squares means, are predicted values from a multiple regression equation containing categorical predictor (factors, i.e., weighted equal to the number of observations in each level) and numerical predictors (covariates, i.e., weighted 
No pain Very severe pain

Figure 18. Example of a Visual Analogue Scale (VAS), a horizontal line, $100 \mathrm{~mm}$ in length, anchored by word descriptors at each end. 
proportional to the number of observations in each level). To estimate adjusted means, the mean value of any covariate is used to estimate the mean response for all combinations of the factors and taking simple means of these estimates over factor levels.

\section{Profile Attractiveness Following Functional Therapy}

The change in profile attractiveness of Class II, division 1 malocclusion patients was measured following functional therapy with a Fränkel regulator and a Harvold activator in a study from New Zealand. Forty-two 11 year old subjects were randomly assigned to an untreated control group (11 males, 6 females), a Fränkel treatment group (8 males, 5 females), or a Harvold activator group ( 7 males, 5 females). The change in profile attractiveness after 18 months of treatment was assessed by panels of dental students, art students, and parents of orthodontic patients using a VAS. Each panel consisted of 30 evaluators with 15 males and 15 females. The initial and the final profiles of each subject were converted to silhouettes and shown as a pair, randomly assigned as either A or B. The examiners were given 20 seconds to decide which profile they preferred and to record the level of attractiveness of their preferred profile using a VAS marked at the ends by the descriptors $0 \%$ and $100 \%$ more attractive. The results of this study showed there was no significant difference between the change in profile attractiveness of the untreated subjects and the subjects treated with the two functional appliances. Neither were there significant differences between the male and female raters or among the panels in their assessments of profile change. Thus, they concluded that when compared to a nontreatment group, treatment with either a Fränkel or a Harvold appliance would not create more attractive profiles (O’Neill et al. 2000).

More recently, Stock et al. from the University of Iowa examined the profile esthetics of 96 growing Class II, division 1 patients treated with either a Herbst appliance or headgear prior to full orthodontic appliances. Profile silhouettes from pre- and posttreatment cephalograms were scored on a 7-point Leikert scale by 10 orthodontic residents and 10 lay people. Both treatment groups were found to have significantly higher mean attractiveness scores posttreatment $(\mathrm{P}<0.05)$, but no significant difference was found at posttreatment between the two treatment groups $(P=0.936)$. Pretreatment scores for the headgear group were scored significantly less than the Herbst group $(\mathrm{P}<0.05)$; and the mean average change scores were also found to be slightly lower in the Herbst group when compared to the headgear group $(\mathrm{P}=0.087)$. An interesting assessment was made by the lack of lower profile scores in the midst of increased skeletal severity. It was concluded from these findings a growing Class II, division 1 patient could achieve an equivalent profile improvement when treated 
with either a Herbst appliance or with headgear prior to fixed appliances (Stock et al. 2006).

Both of the previous studies examined the effects of functional therapy which was followed by full Edgewise orthodontic application in a two-phase process unlike the present study. There has yet to be a study within the orthodontic literature to evaluate profile attractiveness following functional therapy with a MARA, making functional therapy and orthodontic correction a single phase.

\section{Class II, Divison 1 Correction}

Two treatment modalities exercised in the treatment of mandibular retrognathic Class II, division 1 malocclusions include Tweed-Merrifield's standard Edgewise mechanics and functional jaw orthopedic therapy in conjunction with or followed by straightwire mechanics (Graber et al. 2005). The following will discuss the diagnostic considerations, goals and treatment mechanics of the two approaches examined in this study: (1) Tweed-Merrifield standard Edgewise mechanics and (2) functional therapy using a MARA in conjunction with or immediately following straightwire mechanics.

\section{Standard Edgewise Mechanics}

There are three steps in differential diagnosing using modernized TweedMerrifield standard Edgewise mechanics; they are in order: (1) evaluate the face, (2) evaluate the skeletal pattern, and (3) evaluate the teeth. The primary goal of treatment is to improve or maintain facial balance, harmony, and proportion. The four prerequisites for facial balance are taught to include: (1) a soft tissue chin nicely positioned in the facial profile, (2) a lack of severe skeletal convexity, (3) adequate lip fullness as measured by the profile line (Figure 16), which is where the lower lip should lay, and (4) a definite upper lip curl, measuring three to five $\mathrm{mm}$ in depth, and a lower lip curl in harmony with the upper lip. Often too Merrifield's Z angle (Figure 16), the angle formed by the profile line and Frankfort horizontal (Figure 17), is used to quantify balance and harmony of the lower face, having an ideal range of $72^{\circ}$ to $78^{\circ}$ (Merrifield 1966).

In evaluating the skeletal pattern and teeth, Charles H. Tweed developed a diagnostic triangle, termed the Tweed triangle. In developing this he selected 95 subjects, which he felt presented good facial esthetics and balance, and measured their Frankfort-mandibular plane angle (FMA) (Figure 19), incisor-mandibular plane angle (IMPA) (Figure 20), and Frankfort-mandibular incisor angle (FMIA) 


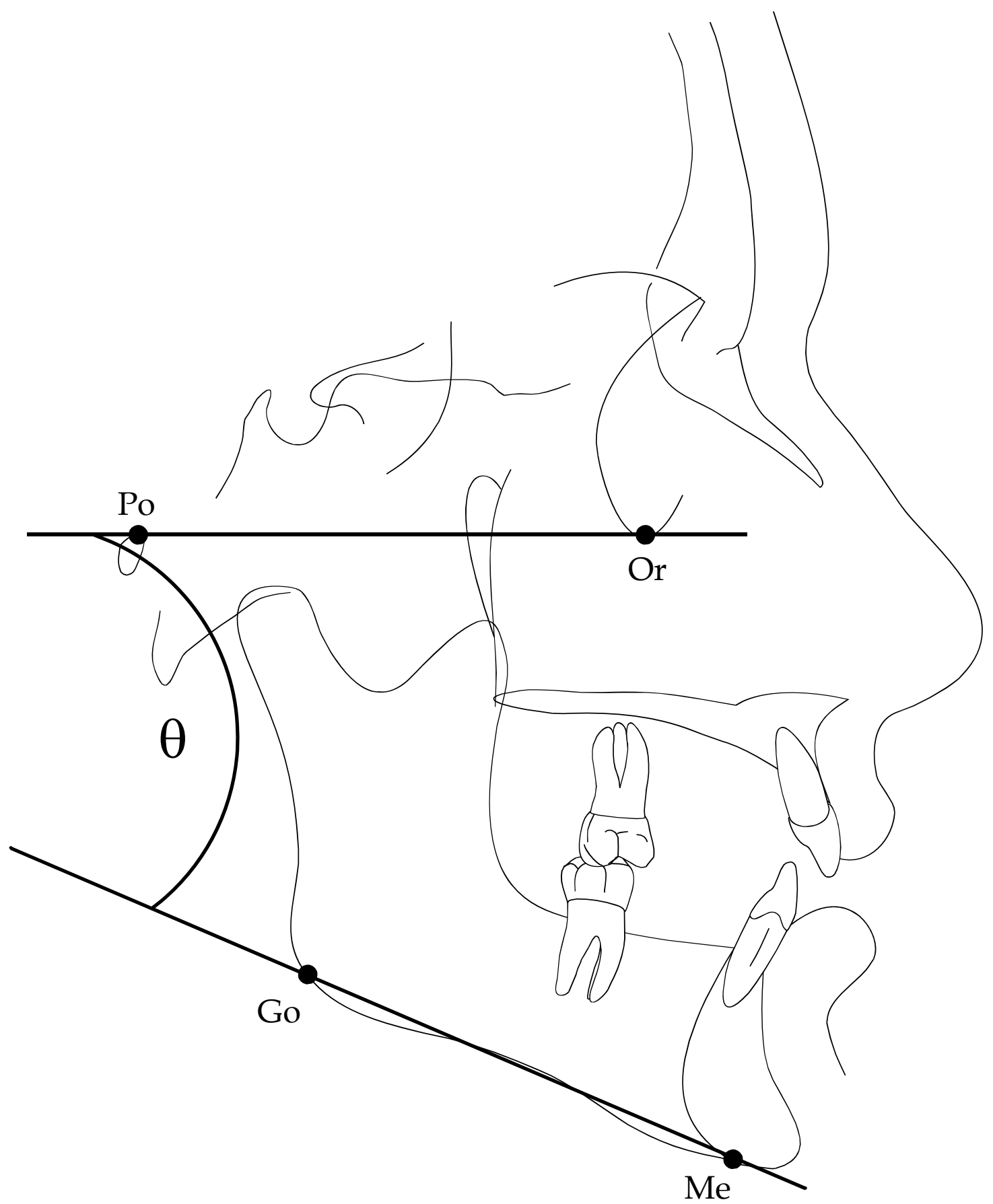

Figure 19. Schematic tracing of a lateral cephalogram showing construction of FMA. This is the angle $(\theta)$ formed by the inferior-anterior intersection of Frankfort Horizontal and the Gonion-Menton line. 


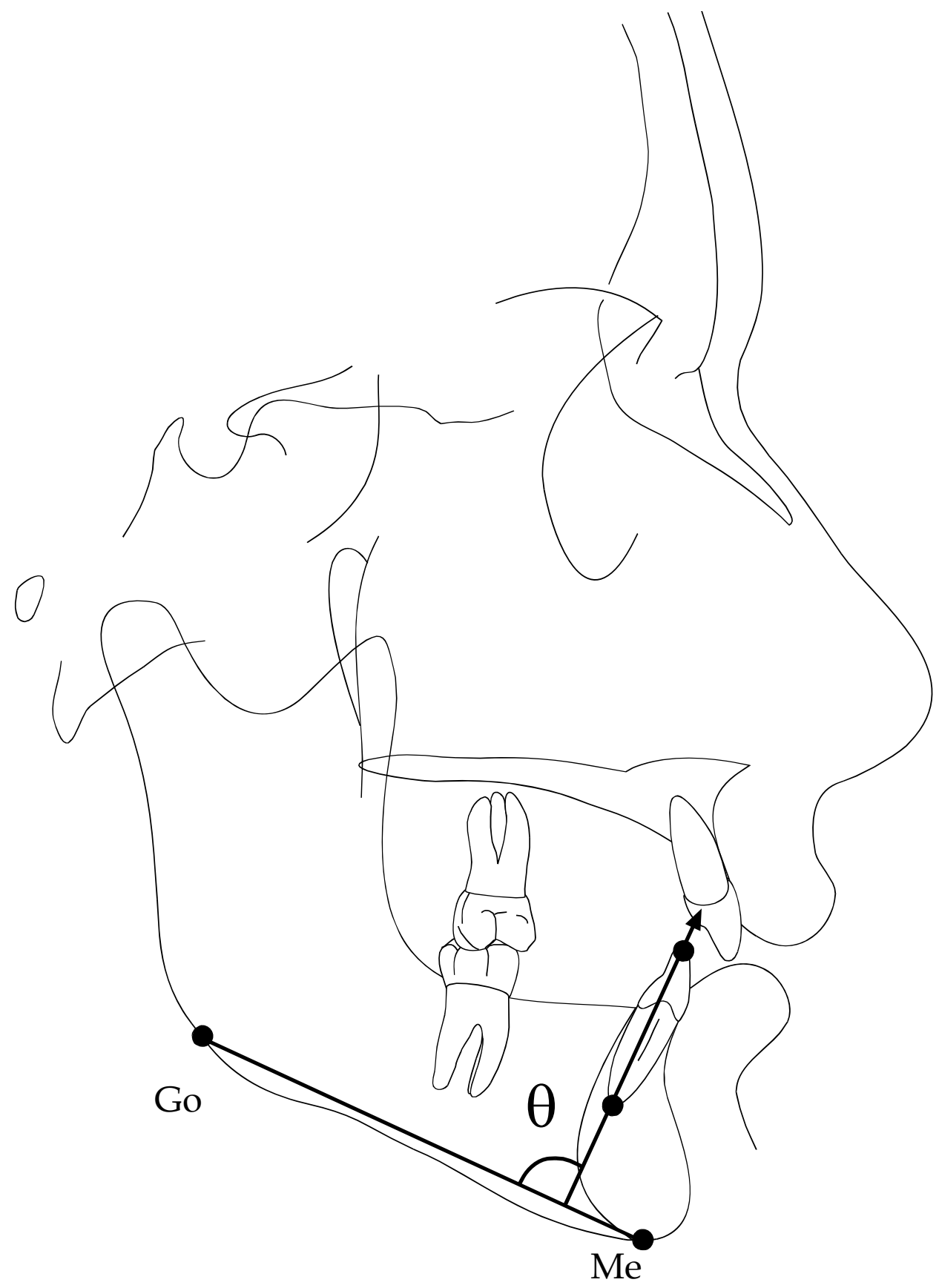

Figure 20: Schematic tracing of a lateral cephalogram showing construction of the angle $(\theta)$ of the mandibular incisor and the mandibular plane (IMPA). 
(Figure 21) which form a triangle. From these averages, Tweed established the guidelines for which he felt the FMIA or the IMPA should be treated to for different values of FMA. If a patient presents with an FMA greater than $30^{\circ}$, the treatment objective for the FMIA should be $65^{\circ}$. In a patient that displays an FMA between $20^{\circ}$ to $30^{\circ}$, goal for FMIA should range from $65^{\circ}$ when the FMA is $30^{\circ}$, to $72^{\circ}$ when the FMA is $20^{\circ}$. For those patients with an FMA less than $20^{\circ}$, the IMPA should not exceed $94^{\circ}$ (Tweed 1966).

In hopes of restoring the face to a more harmonious relationship, Tweed felt it necessary to treat his patients to this diagnostic triangle. The position of the lower incisors within the skeletal frame was believed by Tweed to be detrimental in facial balance and harmony and should be considered, along with the integumental profile, in evaluation of treatment needs (Lindquist 1958). Tweed's view that a correlation exists between balanced facial lines and the position of the mandibular incisors has contributed to his diagnostic triangle becoming a widely used treatment objective (Tweed 1954).

Modernized Tweed-Merrifield standard Edgewise mechanics utilize growth in correcting a Class II, division 1 relationship. This is done by controlling the vertical vectors of force during mechanotherapy allowing for a favorable horizontal "mandibular response," a term used to describe the total effect of both growth and treatment on the sagittal relationship of the mandible and maxilla (Vaden et al. 1994). Utilizing a directional force system, a group of force systems that utilize directional control to precisely position the teeth so they are in harmony with their environment, Tweed-Merrifield standard Edgewise mechanics produce a counterclockwise rotation of the mandible, with the mandibular response, to correct mandibular deficiencies in Class II, division 1 malocclusions (Merrifield 1989; Gebeck 1989).

\section{Mandibular Anterior Repositioning Appliance}

\section{Background}

The MARA appliance was originally created in 1991 by Douglass Toll of Germany. It was then further developed with the help of Jim Eckhart, of Manhattan Beach, CA, Ormco, and AOA laboratory. A newly designed MARA was provided in 1995 for clinical trials and feedback from Eckhart's table clinics at the 1996, 1997 and 1998 AAO meetings were positive, as well as from Toll's 1997 and 1998 AAO lectures. The MARA (Figures 22, 23, and 24) is assembled to be low in bulk, therefore easily tolerated by the patient, and engages the use of stainless steel crowns on the molars with soldered "arms" which help guide the 


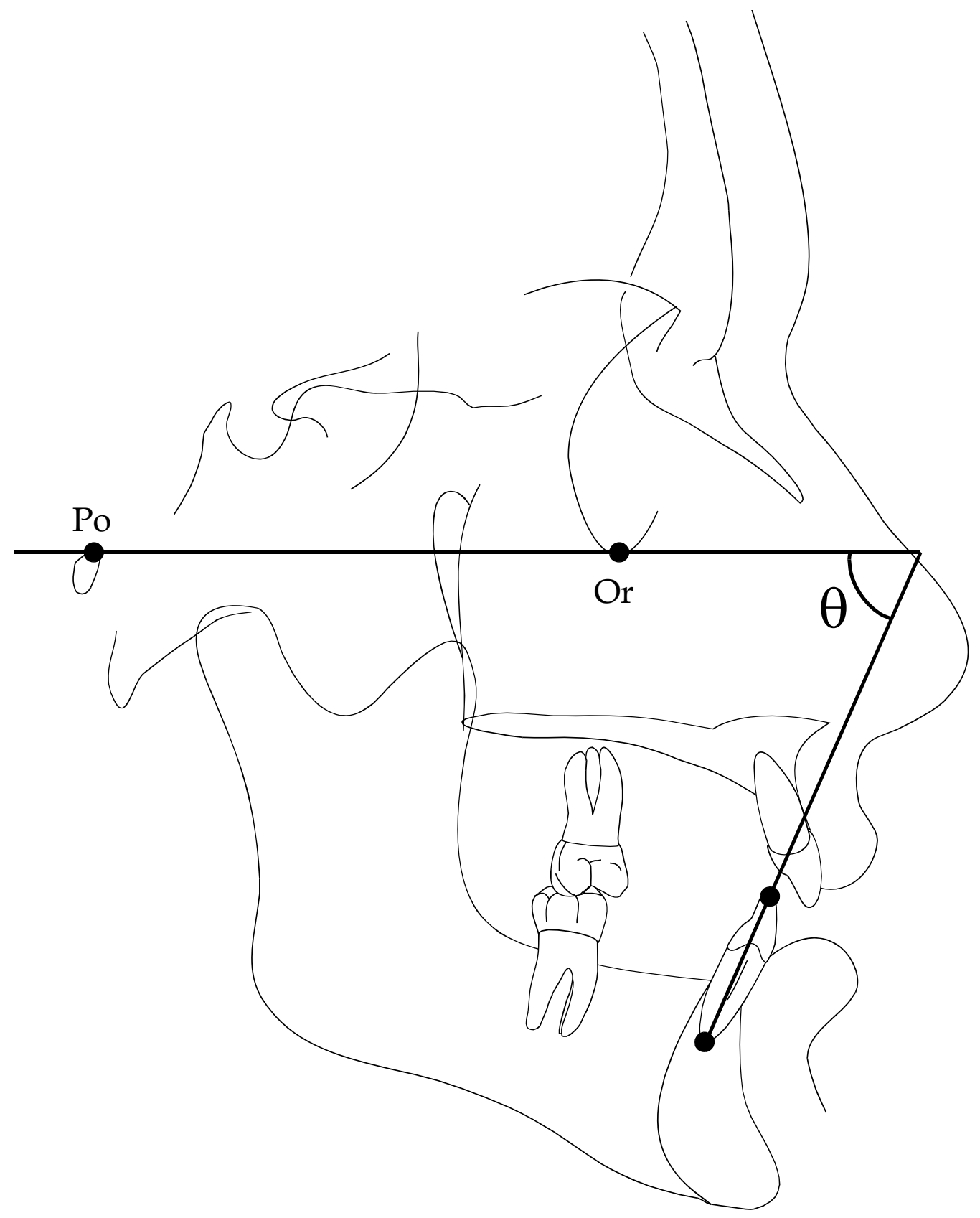

Figure 21. Schematic tracing of a lateral cephalogram showing construction of FMIA angle $(\theta)$. This is formed by the intersection of Frankfort Horizontal and the long axis of the mandibular incisor (L1 apex and L1 incisal edge). 


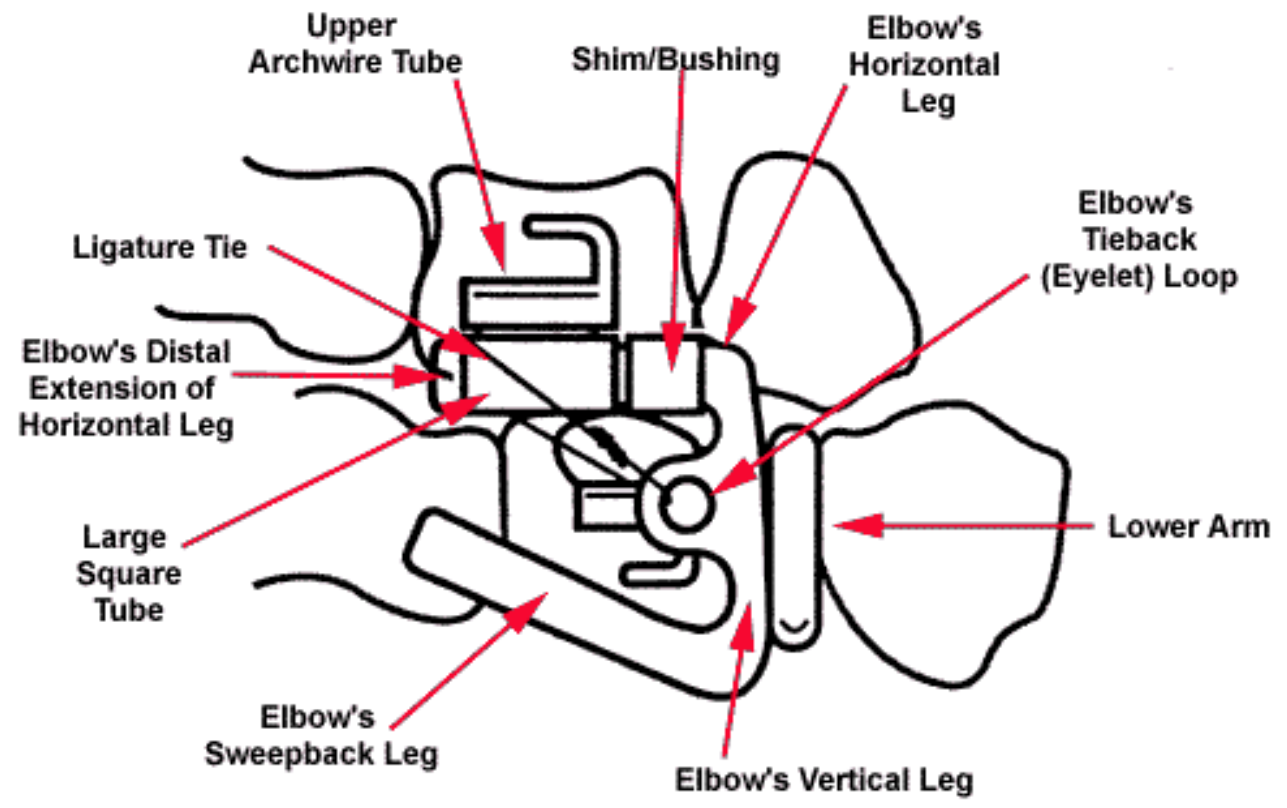

Figure 22. Buccal view and description of MARA.

Modified from Allen-Noble PS, Allesee Orthodontic Appliance/Pro. Clinical management of the MARA. 2002. 


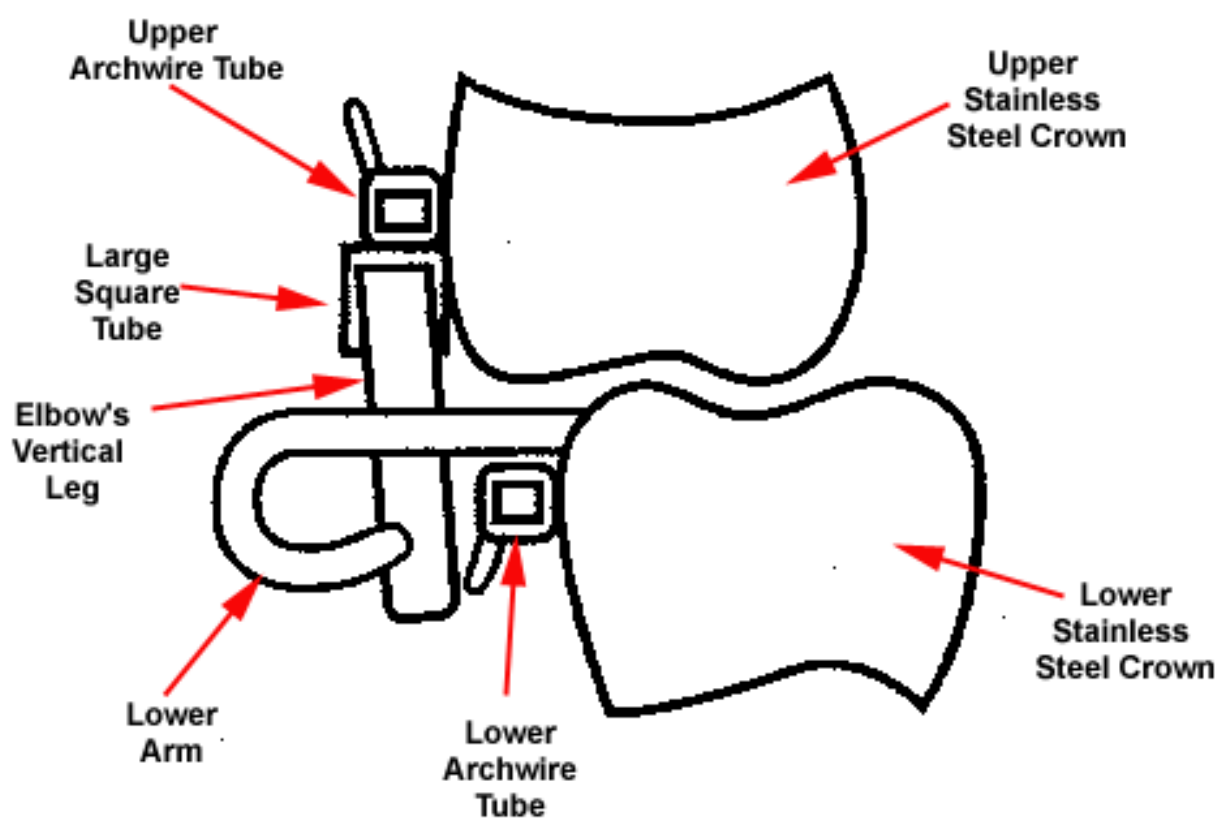

Figure 23. Frontal view and description of MARA.

Modified from Allen-Noble PS, Allesee Orthodontic Appliance/Pro. Clinical management of the MARA. 2002. 


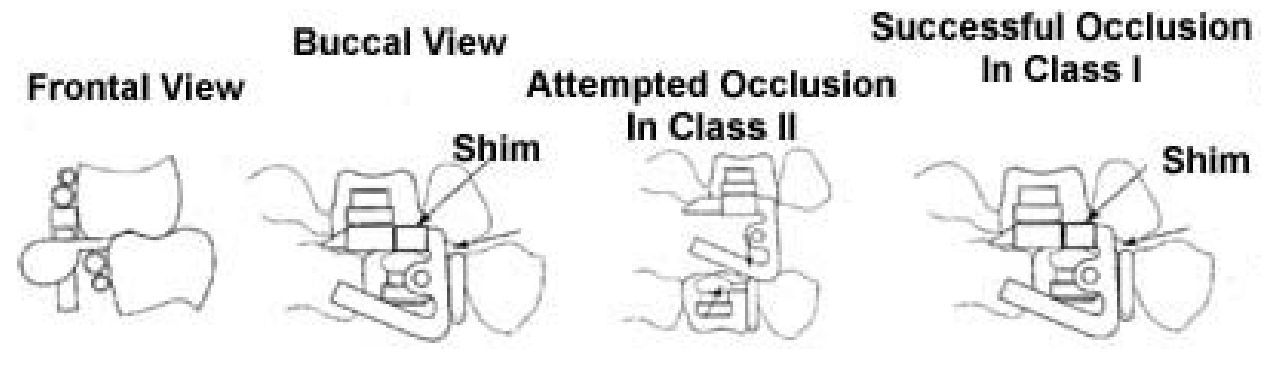

Figure 24. Schematic views of how the MARA works.

Modified from Allen-Noble PS, Allesee Orthodontic Appliance/Pro. Clinical management of the MARA. 2002. 
patient to bite into Class I occlusion as shown in Figure 25 (Allen-Noble 2002; Rondeau 2002).

The MARA works by causing occlusal interferences from a upper first molar stainless steel crown and a buccally attached vertical bar that hits a buccally protruding horizontal bar that extends gingivally from a lower first molar stainless steel crown. This encourages the patient to protrude the mandible forward when biting into occlusion. Supplementary activations of the MARA can be made by the insertion of shims (varying in length) on the horizontal portion of the vertical bar found on the upper first molar, allowing the mandible to be advanced forward incrementally. Over a period of months this forward posture of the mandible is intended to promote forward growth of the mandible (Allen-Noble 2002; Rondeau 2002).

When used in a growing patient to correct Class II malocclusions, a MARA is intended to accelerate the growth of the lower jaw and inhibit the growth of the upper jaw, allowing the mandible to become equal with the maxilla. It is classified as a functional therapy device because it is intended to posture the patient's lower jaw forward, which in turn is intended to promote forward growth and positioning of the mandible by condylar growth, condylar remodeling, reshaping of the fossa, and/ or possible forward rotation of the temporal bone as diagrammed in Figure 26 (Allen-Noble 2002). Class II correction is also aided through the inhibition of maxillary growth and dentoalveolar changes in the upper and lower molars and incisors (Figure 15). These changes are similar to what is often seen in Herbst therapy, but with less vertical opening in the mandible due to the use of full coverage crowns (AllenNoble 2002).

Unlike other functional correctors, the MARA can be worn in conjunction with Edgewise appliances, which is a distinction from most Class II appliances (Graber et al. 2005). It is also a tooth-borne appliance that positions the mandible forward into a Class I relationship, but does not connect the maxilla and mandible through pistons, coils, springs, wires, or other mechanisms as do other functional appliances. Advantages of the MARA include immediate profile enhancement in response to forward positioning of the mandible and chin, freedom from patient compliance since the appliance is fixed, and the added ability for conjunctive wear of orthodontic appliances with MARA functional therapy (Allen-Noble 2002).

It is suggested that the MARA be used in patients in the late mixed dentition, due to the insufficient cheek room during the early mixed dentition. It also is indicated for treatment of adults due to the believed remodeling capacity of the glenoid fossa and condylar head. Working well with all types of dental 


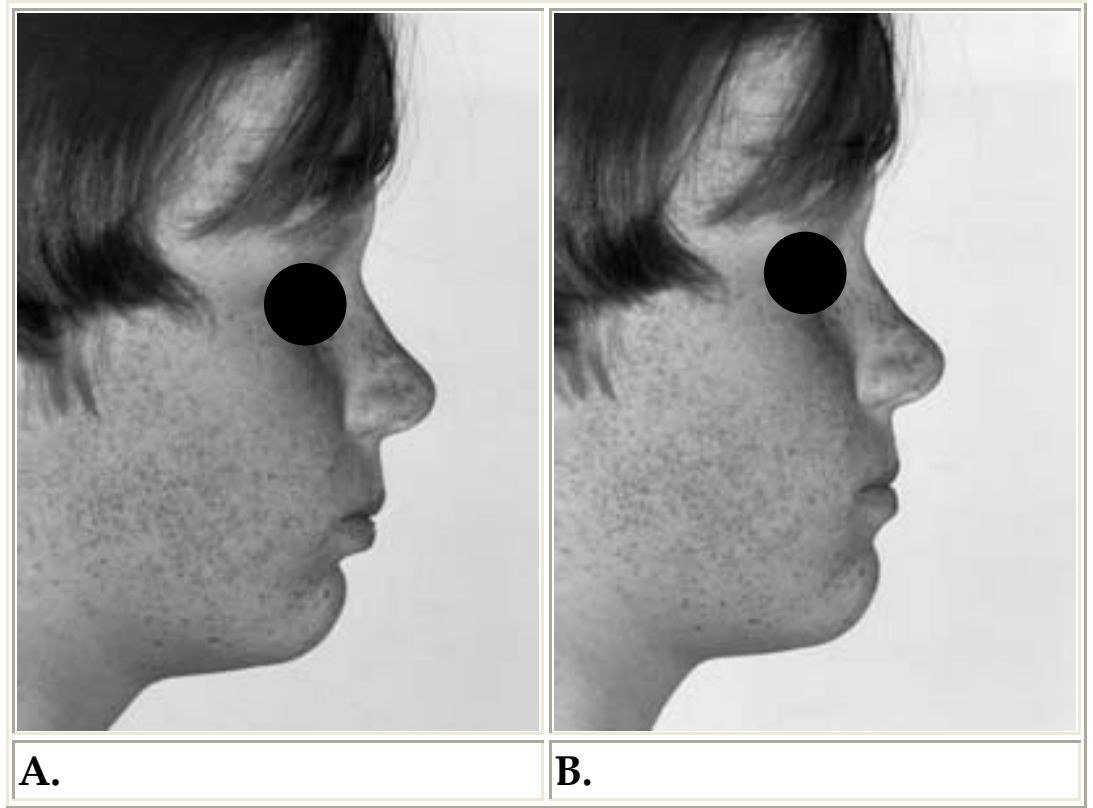

Figure 25. (A.) Patient with a typical Class II profile.

(B.) Patient with lower jaw positioned forward and edge-toedge with MARA.

Modified with permission from Allen-Noble PS, Allesee Orthodontic Appliance/Pro. Clinical management of the MARA. 2002. 


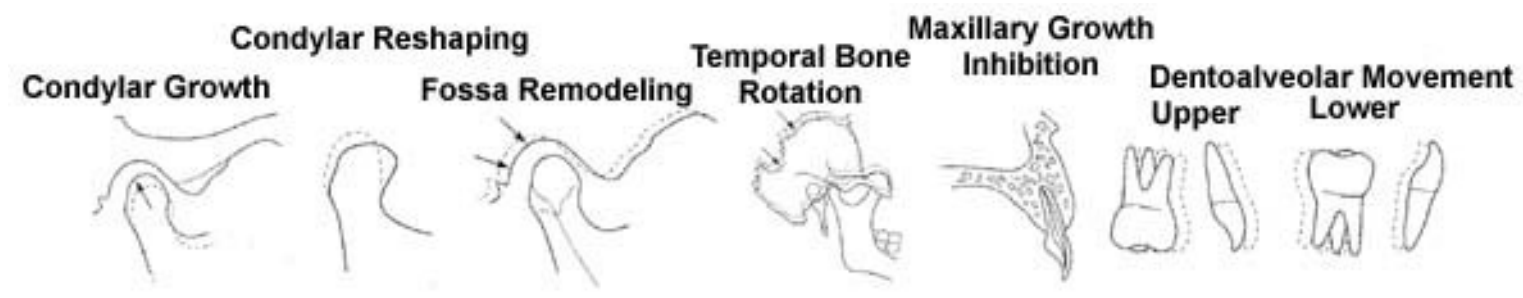

Figure 26. Speculative changes produced by the MARA. Initial tracings are designated by the dotted lines, while the solid-line tracings designate changes produced by the MARA.

Modified from Allen-Noble PS, Allesee Orthodontic Appliance/Pro. Clinical management of the MARA. 2002. 
and skeletal Class II malocclusions, it is especially recommended in deepbite, brachyfacial cases. The MARA may not be effective in cases with extremely short mandibular rami, due to insufficient growth in this facial form, or in cases with high mandibular plane angles due to the increase in vertical facial height with Class II correction, unless measures are taken to prohibit this (e.g., headgear wear at night) (Allen-Noble 2002).

\section{Skeletodental and Cephalometric Findings}

Studies have documented successful mandibular orthopedic effects when using the MARA appliance in the correction of Class II malocclusions. In a study by Pangrazio-Kulbersh and others, the treatment effects of a MARA on 30 patients with Class II malocclusions were examined. Their sample consisted of a treatment group of 18 females (mean age of 11.3 years) and 12 boys (mean age of 11.2 years). The treatment group was compared to a control group of 21 nontreated Class II subjects for whom longitudinal cephalometric records were available. The determined treatment effects on the skeletal and dental relationships included molar correction due to distal repositioning of maxillary molars by an average of $1.1 \mathrm{~mm}$ in the MARA group, while the control group had a mesial migration of $1.3 \mathrm{~mm}$, so the total treatment effect of the MARA was $2.4 \mathrm{~mm}$ of maxillary molar change. Further molar correction was due to the forward movement of mandibular molars by $1.2 \mathrm{~mm}$ in the experimental group, whereas the control group average only $0.5 \mathrm{~mm}$ of mandibular molar mesial drift, so the total treatment effect of the MARA was $0.7 \mathrm{~mm}$ of forward mandibular movement. An increase in horizontal mandibular length when measured cephalometrically from Condylion to Gnathion was an annual average increase of $4.8 \mathrm{~mm}$ in the experimental group but only $2.1 \mathrm{~mm}$ in the control group, which produced a net annual average increase in mandibular length of $2.7 \mathrm{~mm}$ in the MARA group; and lastly there was an average increase of $4.0 \mathrm{~mm}$ in posterior facial height annually in the MARA group compared to $1.3 \mathrm{~mm}$ annually in the control group when measured from Condylion to Gonion. The examiners suggest that this mandibular growth agrees with previous studies due to the use of stainless steel crowns in the MARA appliance causing the condyle to be positioned inferiorly in the glenoid fossa during occlusion, theoretically stimulating condylar growth in a superior, posterior direction, and in turn increasing posterior facial height (Pangrazio-Kulbersh et al. 2003). 


\section{CHAPTER III MATERIALS AND METHODS}

In the present study the perceived profile attractiveness of Class II, division 1 patients prior to and following orthodontic treatment with either (1) premolar extractions and standard Edgewise mechanics or (2) with a MARA, a functional device, with preangulated Edgewise appliances was investigated. The purpose of this section is to describe the materials and methods used in this study.

\section{$\underline{\text { Research Design }}$}

This was a retrospective study, meaning the study investigated patients previously treated using existing patient records obtained for purposes other than research (Hess 2004). Information from patient records of completed cases, consisting of pretreatment and posttreatment lateral photographs and cephalograms, were gathered to determine if either treatment group resulted in a more preferable profile.

This was also a matched-pair design study where two groups, composed of individually matched pairs, were matched according to factors that might cause confounding problems, thereby helping to control for erroneous findings and statistical misinterpretations (Rosner 2000). In this study the MARA and Edgewise groups were matched according to seven factors to strengthen results and prevent misrepresented findings; these factors consist of race, sex, age at onset of treatment, Frankfort mandibular plane angle (FMA), Sella-Nasion-Point A (SNA) angle, Sella-Nasion-Point B (SNB) angle, and Point A-Nasion-Point B (ANB) angle. Both the ANB angle, indicating the magnitude of skeletal jaw discrepancy (Graber et al. 2005) and FMA, an angular skeletal value that is often used for differential diagnosis and to better understand skeletal relationships (Moyers et al. 1980; Merrifield 1996), were chosen to better coordinate the two treatment groups, making them more comparable.

All too commonly in the orthodontic literature groups are labeled "matched" when, in fact, there merely have similar group characteristics. "Matched" is used in its correct statistical sense in the present study, where each individual subject in one sample (the MARA group) was matched demographically and cephalometrically to a subject in a second sample (labeled the Edgewise group). As a result, the two sample sizes are directly comparable and, more importantly, repeated-measures statistical designs (e.g., Winer et al. 1991) can be used in place of less efficient group comparison tests. A repeated- 
measures analysis of variance (or, equivalently, a paired t-test) matches each case from one group with a case from the second group, so the difference between the groups is tested as a function of the standard error of the mean difference. This measure of variability is always smaller than the more common group comparison t-test (or factorial ANOVA), so it is more likely to discover a difference if one actually exists. In other words, a paired t-test is less likely to produce a type II statistical error (i.e., acceptance of a false null hypothesis) and is more efficient.

\section{Cephalometrics}

At the heart of this thesis is the question of whether the use of a MARA in combination with Edgewise mechanics yields a more esthetic facial profile than Edgewise treatment alone. Because the integumental profile depends considerably on the underlying bony (skeletodental) support, it follows that the obvious way an orthodontist can improve the patient's soft-tissue profile is to improve the harmony of the underlying supporting structures. So too, it is relevant to the design of this study that the starting conditions of the two treatment modalities be as comparable as possible. The purpose of this section is to document that key cephalometric conditions were comparable at the start of treatment.

The author devoted considerable time collecting data to provide a casecontrol matched sample of cases treated orthodontically. The commonly encountered orthodontic research design is to use a group comparison design, where the average characteristics of the two groups are argued to be comparable. This is expedient, but statistically much less efficient than comparing matched samples. In contrast, we first evaluated the MARA sample and matched an Edgewise case to each based on what we deemed key characteristics of the skeletal malocclusion.

For each MARA case (at about 12.7 years of age), the pool of Edgewisetreated cases was reviewed to provide a close match for the four cephalometric variables. Operationally, we felt there was adequate precision if all the four variables "matched" within 1 or 2 degrees of the MARA case accounting for the expected sex-specific age changes (Riolo et al. 1974). Pointedly, we made no special effort to match for dental characteristics of the malocclusion, though all cases began treatment as Class II, division 1 malocclusions. 
Table 1 lists the sample means for the two groups along with the results of pairing design t-tests (Woolf 1968). Results are confirmatory that our efforts to match the Edgewise cases with cephalometrically quite-similar MARA cases was successful.

The biggest difference between the two groups was for FMA, which was, on the average, less than $2^{\circ}$ different between the matched pairs. The Edgewise sample had just a slightly steeper mandibular plane angle at the start of treatment.

For completeness, we followed the course of these four cephalometric variables through to the end of treatment (Table 2). Again by paired t-test, FMA, SNA, and SNB were each statistically equivalent at the end of treatment. Indeed, the means for FMA were $25.7^{\circ}$ in both samples. Inspection shows that, while not different statistically, the MARA sample was treated to a slightly higher SNA angle $\left(79^{\circ}\right.$ vs. $\left.78^{\circ}\right)$, while SNB was virtually indistinguishable $\left(76^{\circ}\right.$ for both means). These slight differences contributed to the marginally-significant differences in ANB at the end of treatment $(P=0.03)$, where mean ANB was $3^{\circ}$ for the MARA sample and just over $2^{\circ}$ for the Edgewise sample. Of course, both of these means are well within normal limits. For example, Steiner (1953) lists the ideal ANB as $2^{\circ}$, and Riolo et al. (1974) found the normative value to be $3^{\circ}$.

An important consideration in these comparisons is how large the orthodontic corrections were. (In fact, the cephalometric changes are some unknowable combination of treatment plus growth.) Table 3 lists statistics for the in-treatment changes. On average, FMA remained stable, with mean changes of $-0.1^{\circ}$ to $0.2^{\circ}$. SNA decreased in both samples, probably due to Class II mechanics, but the change was twice as big in the Edgewise sample $\left(-3^{\circ} v s .-1^{\circ}\right)$, which is significant statistically $(P=0.009)$. SNB did not change in the Edgewise sample, but it increased about one-half degree in the MARA sample; statistically SNB did not differ in the two samples $(\mathrm{P}=0.34)$. ANB decreased in both samples, but almost twice as much in the Edgewise group $(\mathrm{P}=0.02)$.

Incidentally, the change in ANB was statistically significant in both samples as gauged from one-sample t-tests $(P<0.01)$. Table 3 shows that more of the reduction of ANB was due to maxillary restraint in the conventional Edgewise sample and more to mandibular enhancement with the MARA device. 
Table 1. Results of paired t-tests for cephalometric differences at the pretreatment examination.

\begin{tabular}{lcccc}
\hline Statistic & FMA & SNA Angle & SNB Angle & ANB Angle \\
\hline Edgewise & 25.783 & 80.717 & 75.700 & 5.017 \\
MARA & 25.433 & 80.333 & 75.567 & 4.767 \\
Difference & 0.350 & 0.383 & 0.133 & 0.250 \\
SE & 0.178 & 0.969 & 0.992 & 0.333 \\
n & 30 & 30 & 30 & 30 \\
t-test & 1.97 & 0.40 & 0.13 & 0.75 \\
df & 29 & 29 & 29 & 29 \\
P Value & 0.0589 & 0.6953 & 0.8941 & 0.4586 \\
\hline
\end{tabular}


Table 2. Results of paired t-tests for cephalometric differences at the posttreatment examination.

\begin{tabular}{lcccc}
\hline Statistic & FMA & SNA Angle & SNB Angle & ANB Angle \\
\hline Edgewise & 25.683 & 77.900 & 75.550 & 2.350 \\
MARA & 25.650 & 79.117 & 76.017 & 3.100 \\
Difference & 0.033 & -1.217 & -0.467 & -0.750 \\
SE & 0.404 & 0.813 & 0.934 & 0.336 \\
n & 30 & 30 & 30 & 30 \\
t-test & 0.08 & -1.50 & -0.50 & -2.23 \\
df & 29 & 29 & 29 & 29 \\
P Value & 0.9348 & 0.1454 & 0.6211 & 0.0336 \\
\hline
\end{tabular}


Table 3. Results of paired t-tests for cephalometric differences for the in-treatment changes.

\begin{tabular}{lcccc}
\hline Statistic & FMA & SNA Angle & SNB Angle & ANB Angle \\
\hline Edgewise & -0.100 & -2.817 & -0.150 & -2.667 \\
MARA & 0.217 & -1.217 & 0.450 & -1.667 \\
Difference & -0.317 & -1.600 & -0.600 & -1.000 \\
SE & 0.3884 & 0.56953 & 0.61607 & 0.40115 \\
$\mathrm{n}$ & 30 & 30 & 30 & 30 \\
t-test & -0.82 & -2.81 & -0.97 & -2.49 \\
df & 29 & 29 & 29 & 29 \\
P Value & 0.4215 & 0.0088 & 0.3382 & 0.0186 \\
\hline
\end{tabular}




\title{
Group Comparisons
}

\author{
MARA Group
}

\section{Diagnostic Considerations}

Records for the MARA group were obtained from the office of Dr. Joe L. Wasson, Memphis, Tennessee, who treated all of these subjects. According to Dr. Wasson, patients typically respond well to particular functional therapy devices when certain diagnostic factors are considered. He employs a three-tier treatment strategy for Class II malocclusion correction based on certain diagnostic characteristics, and he classifies MARA therapy in his third treatment tier. The following comments describe his tiered strategy for Class II correction.

The first tier typically is treated with a Fränkel appliance. According to Dr. Wasson, patients that typically respond well to Fränkel appliance therapy often present in the early mixed dentition (7 to 10 years of age), with high mandibular plane angles, openbite tendencies, and deleterious oral habits (e.g., tongue thrusts). In addition, these patients tend to have large overjets and constricted or underdeveloped dental arches. Treatment of these patients typically occurs in a two-phase regimen. The first phase includes Fränkel II wear, approximately 24 hours a day for approximately one year. Patients are seen every two months for evaluation that requires relatively little chair time. Between phase 1 and phase 2 treatments, patients are instructed to continue Fränkel wear at night-only for retention purposes. When the permanent canines and premolars emerge, full appliances are placed.

The second tier of Dr. Wasson's treatment strategy is intended for patients who have low mandibular plane angles and deepbite tendencies. These patients are somewhat older than the Fränkel patients but are still in the mixed dentition prior to the emergence of the first and second premolars (approximately 8 to 11 years of age). According to Dr. Wasson, these patients respond well to Bionator therapy, as a first phase of treatment, worn full time for approximately one year, and they are seen every two months for observation, requiring little chair time by the orthodontist. This is followed by night time wear for retentive purposes until the patient is ready for the second phase of treatment with full appliances, after the emergence of the permanent canines and premolars.

The third tier of Class II treatment involves the subjects used in the present study. This tier is designed for Class II, division 1 patients in the early permanent dentition (approximately 11 to 14 years of age). The diagnostic characteristics most commonly found in this tier include patients with an 
average Frankfort-mandibular plane angle, a skeletal discrepancy caused by mandibular deficiency, and all permanent teeth fully erupted (often excluding second and third molars). This group of patients often is treated with a fixed functional appliance (e.g., the MARA or Herbst appliance), which often is worn for one year in conjunction with or immediately prior to full appliances. Patients are seen every six to eight weeks, requiring limited chair time for check-ups and the occasional advancement or repair.

\section{Sample}

The MARA group studied here consisted of 21 males and 16 females consecutively treated with a MARA, for a total of 37 subjects. These subjects were chosen for MARA treatment after diagnostic considerations and were treated in accordance with or prior to full preangulated Edgewise appliances. The inclusion criteria for subjects in this group consisted of:

1. records containing pre- and posttreatment digital lateral photographs, as well as pre- and posttreatment lateral cephalograms;

2. pretreatment presentation of Class II, division 1 malocclusions due to a skeletal discrepancy caused by mandibular retrognathism;

3. treatment consisting of orthopedic functional therapy using a MARA in combination with preadjusted Edgewise appliances; and

4. fully erupted permanent dentition (often excluding second and third molars).

Because of ethnic differences in the integumental profile of Caucasian and African American females (e.g., Sutter and Turley 1998), the single African American female subject was excluded from this group. Subjects with unfocused lateral photographs were also excluded.

After the selection criteria were instituted, the MARA group was left with 34 subjects (19 males, 15 females). To balance the number of males and females, 15 males were randomly chosen from among the 19. This left the sample with 30 subjects (15 males and 15 females), a mean pretreatment age of 12.7 years (13.0 years for males, 12.4 years for females) and a range of 10.6 to 15.2 years (10.6 to 15.2 years for males, 11.4 to 14.5 years for females).

\section{Edgewise Group}

The standard Edgewise subjects were chosen from patients treated in the Department of Orthodontics, The University of Tennessee Health Science Center, Memphis, Tennessee, and from the office of Dr. James L. Vaden, Cookeville, 
Tennessee. This group was matched according to the five factors discussed above. The criteria for inclusion for this group consisted of:

1. records containing pre- and posttreatment lateral photographs, as well as preand posttreatment lateral cephalograms;

2. American whites, matched by sex to paired complement in the MARA group;

3. pretreatment presentation of Class II, division 1 malocclusions with insignificantly different ANB measurements of matched counterpart;

4. statistically insignificant differences in FMA measurements and age at treatment onset when compared to matched subjects; and

5. treatment consisting of extractions of either upper first premolars and lower second premolars, upper first premolars only, or all first premolars, and standard Edgewise mechanics.

The matched group of standard Edgewise subjects included 30 subjects (15 males, 15 females) with a mean pretreatment age of 13.1 years (13.2 years for males, 12.9 years for females) and a range of 11.1 to 15.3 years (11.8 to 14.5 years for males, 11.1 to 15.3 years for females).

\section{Esthetic Evaluation}

Since (1) the soft-tissue outline was unclear, or burned-out, on many of the pretreatment and posttreatment cephalograms and (2) only lateral photographic images of the soft-tissue outline were available for all subjects, it was decided that silhouettes would provide the best presentation of the profiles for the present study. With the unclear influence of sex on panel ratings, both male and female observers (consisting of 15 orthodontists, 10 lay people and 10 graduate orthodontic students), were used for this study. And lastly, the evaluations were measured using a visual analogue scale (VAS).

\section{Silhouette Evaluation}

Profile silhouettes were chosen for rating the profiles, rather than lateral photographs, to avoid subjective considerations and eliminate aspects that may influence the observers, such as hair color and hair style, skin complexion, makeup, or age (Shelly et al. 2000; Spyropoulos and Halazonetis 2001; Mergen et al. 2004). In turn, the silhouettes eliminated any distracting extrinsic or intrinsic variables that could influence the observer's esthetic rating, allowing him or her to focus on the outline of the facial profile. Although previously anticipated, Spyropoulos and Halazonetis (2001) verified the influence that inherent factors that occur in photographs possess in biasing ratings of profile esthetics. They 
evaluated lateral photographs that had been altered but with identical profile outlines. Photographs were documented not to be good determinants of profile esthetics, which supports the use of silhouettes in profile evaluations to eliminate influences from other facial features.

The solid black silhouette images (Appendix) were generated from pretreatment and posttreatment digitized lateral photographs using Adobe Photoshop 6.0 (Adobe Systems Incorporated, San Jose, CA) at a resolution of 340 pixels per inch (ppi). For this study each digital image was rotated, if necessary, in Adobe Photoshop 6.0 to position the Frankfort plane (Figure 27) horizontally. Frankfort plane is a line used in anthropometry that passes through Tragion (Tr), the notch at the top of the tragus of the ear, to soft-tissue Orbitale (Or), the inferior border of the bony orbit. This reference plane was adopted in 1882 by an international congress of anatomists and physical anthropologists that met in Frankfort-am-Main, Germany, as the best representation of the natural orientation of the head (Proffit et al. 2007), and it is still employed as a reference plane in anthropometric and orthodontic analyses.

Since orthodontic treatment influences the lower third of the face, the silhouettes were digitally cropped superiorly at soft tissue Nasion $\left(\mathrm{Na}^{\prime}\right)$, omitting eyelashes and eyebrows, and inferiorly at the Throat point (T), a soft tissue point tangent to the cerviomental angle formed by the lower border of the mandible and vertical plane of the neck (Worms et al. 1976; Shelly et al. 2000; Mergen et al. 2004). Careful to maintain the original vertical and horizontal proportions of the silhouette, the height and width ratios of each image were locked prior to standardizing the height of all the images, meaning the width of the images was automatically changed according to the proportional change in height. Then each canvas size's width was standardized to center the silhouette by manually adjusting the amount of white presented anterior to the profile (on the right) and black presented posteriorly (on the left). This standardized each image in height and width at $340 \mathrm{ppi}$, without altering the vertical and horizontal proportions of the image.

\section{Observer Panel Compositions}

Three panels of observers were used, namely 15 experienced orthodontists (10 male, 5 female), 10 orthodontic graduate students ( 7 male, 3 female), and 10 lay people ( 5 male, 5 female). Observers were asked to score the level of profile attractiveness from the silhouette profiles taken before and after orthodontic treatment. Observers also were asked to select the profile that they preferred (from each pair of pre- and posttreatment silhouettes) and to indicate the intensity of their preference. The observers were not informed that they were 


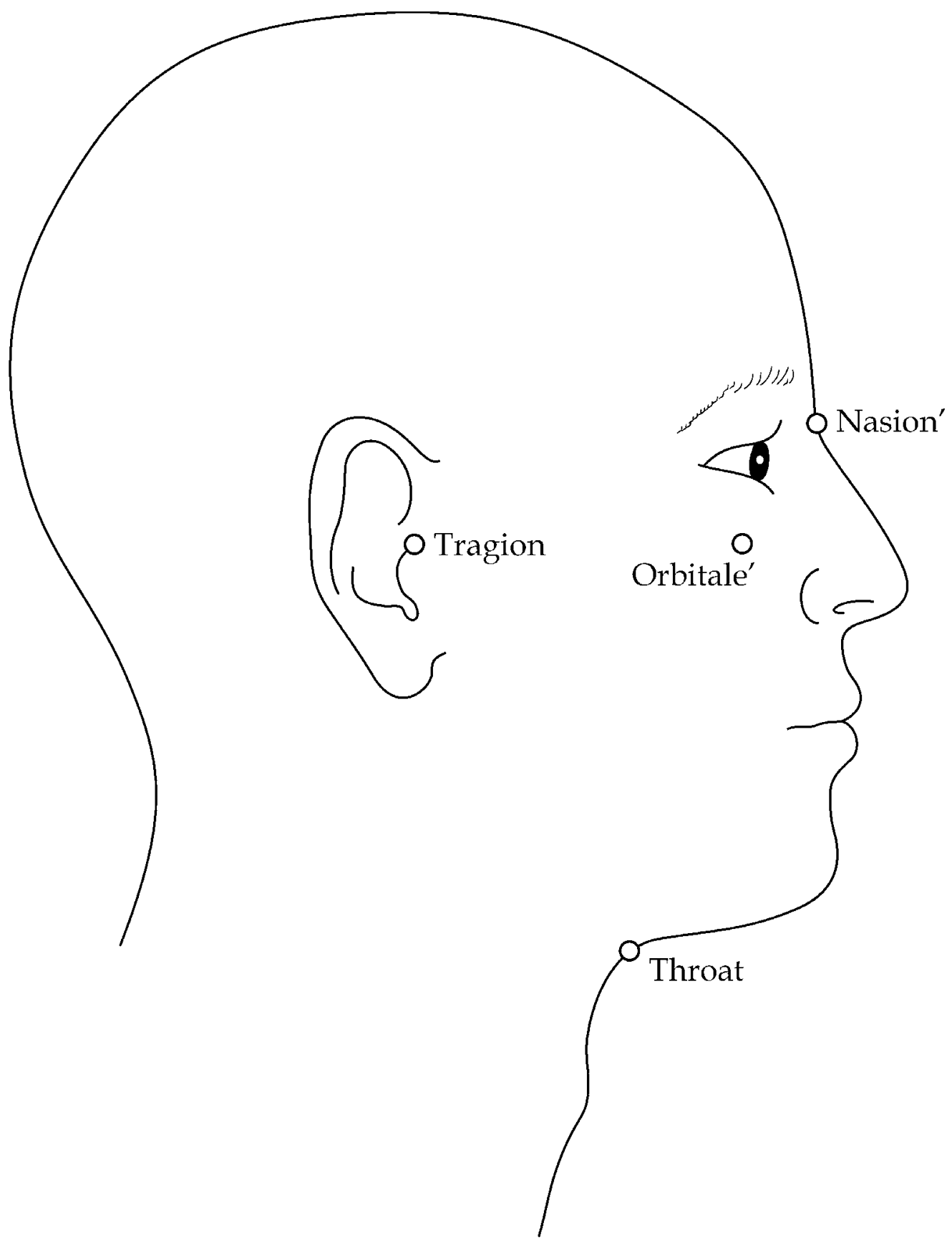

Figure 27. Schematic showing locations of the integumental landmarks used in this study. Frankfort plane is drawn through Tragion, the notch just above the tragus of the ear, and Orbitale', the inferior border of the bony orbit. 
evaluating two different treatment methods or that all subjects were American whites.

The panel made of 15 experienced orthodontists (10 male and 5 female) ranged in age from 30.3 to 70.3 years ( 30.8 to 70.3 years for males, and 30.3 to 53.6 years for females), with a mean of 47.7 years ( 51.2 years for males, and 40.8 years for females). Fourteen members of this panel were American whites, with the other one member being Asian-American. The years of private practice experience for the panel averaged 15.9 years (20.2 years for males, and 7.5 years for females) and ranged from 1.5 to 45 years ( 1.5 to 45 years for males, and 1.5 to 16 years for females). One of the panel members stated their preferred treatment technique was standard Edgewise mechanics, 13 preferred preangulated Edgewise mechanics ("straightwire"), one stated to not have a preferred treatment technique. Thirteen of the experienced orthodontists claimed to use extraoral traction devices (e.g., facebow headgear and high-pull J-hook headgear), and eleven claimed to use functional appliances (e.g., the Bionator, the Fränkel appliance, the Herbst appliance, and the MARA) within their armamentarium. Only two of the panel members claimed to have clinical experience with a MARA.

The panel of 10 orthodontic graduate students ( 7 male and 3 female) ranged in age from 23.1 to 35.8 years (26.4 to 35.8 years for males, and 23.1 to 28.2 years for females), with a mean of 28.8 years (29.9 years for males, and 25.9 for females). All of the members on this panel were American whites, with the exception of one which was Asian-American, and nine claimed to have previously had some type of orthodontic treatment. The amount of graduate orthodontic education varied for this panel with three members ( 2 male and 1 female) in their third year, three members (all male) in their second year, and four members ( 2 male and 2 female) in their first year of orthodontic graduate work.

The panel of 10 lay people ( 5 male and 5 female), of which six ( 1 male and 5 female) claimed to have had some type of orthodontic treatment in the past, ranged in age from 31.3 to 78.9 years (31.3 to 78.9 years for males, and 37.1 to 59.0 years for females), with a mean of 55.2 years (58.0 years for males, and 52.4 years for females). All 10 members of this panel had received a bachelor's degree (of which three had completed a Master's degree, two had completed a Doctorate in Medicine, and one had complete a Doctorate in Philosophy). 


\section{Measuring Technique}

The Visual Analogue Scale (VAS) assessment rating method was chosen for this study given its reproducible and accurate assessment of panel ratings (Howells and Shaw 1985; Shaw et al. 1985; Proffit et al. 1992; Bowman and Johnston 2000; O'Neill et al. 2000; Mergen et al. 2004). It was also chosen for its unproblematic and rapid measurability, as well as the lack of excessive explanation required for its use (Wewers and Lowe 1991).

The observers were asked to evaluate the randomized pretreatment and posttreatment profile silhouettes of the MARA and Edgewise groups using an unmarked horizontal VAS, $100 \mathrm{~mm}$ in length and anchored by the descriptors 0 (very unattractive) and 100 (very attractive) at right angled stops (Figure 28). Observers marked all VAS scores on a provided document after detailed instructions on properly marking the VAS were provided in the tutorial. For this evaluation the randomized silhouettes were individually presented in the center of the presentation screen.

Next, to determine the amount of profile change each observer was first asked which profile they preferred when both the pretreatment and posttreatment silhouettes were presented simultaneously in random order (pretreatment on the left and posttreatment on the right, or posttreatment on the left and pretreatment on the right). Then observers were asked to rate the intensity of their preference using a VAS scale. This VAS was unmarked, horizontal, $100 \mathrm{~mm}$ in length, and anchored by the descriptors $0 \%$ and $100 \%$ more attractive at right angled stops (Figure 29). If the pretreatment silhouette was chosen as the preferred one (i.e., the facial profile was better before treatment), the scale measurement was given a negative value; if the posttreatment silhouette was selected as the more preferred one (i.e., the facial profile was better after treatment), the measurement was given a positive value. Therefore, the esthetic change from pretreatment to posttreatment was measured on a 100point scale ranging from -50 (pretreatment silhouette preferred as $100 \%$ more attractive) through zero (no change) to +50 (posttreatment silhouette preferred as 100\% more attractive).

\section{Evaluation Format}

The silhouettes were presented in the form of a Microsoft (Microsoft, Redmond, WA) PowerPoint presentation, distributed on a compact disc (CD) to the 35 observers. A slide-show tutorial was provided with (1) directions, (2) practice examples, and (3) detailed instructions on properly marking a VAS were given to familiarize raters with the format of the evaluation. 


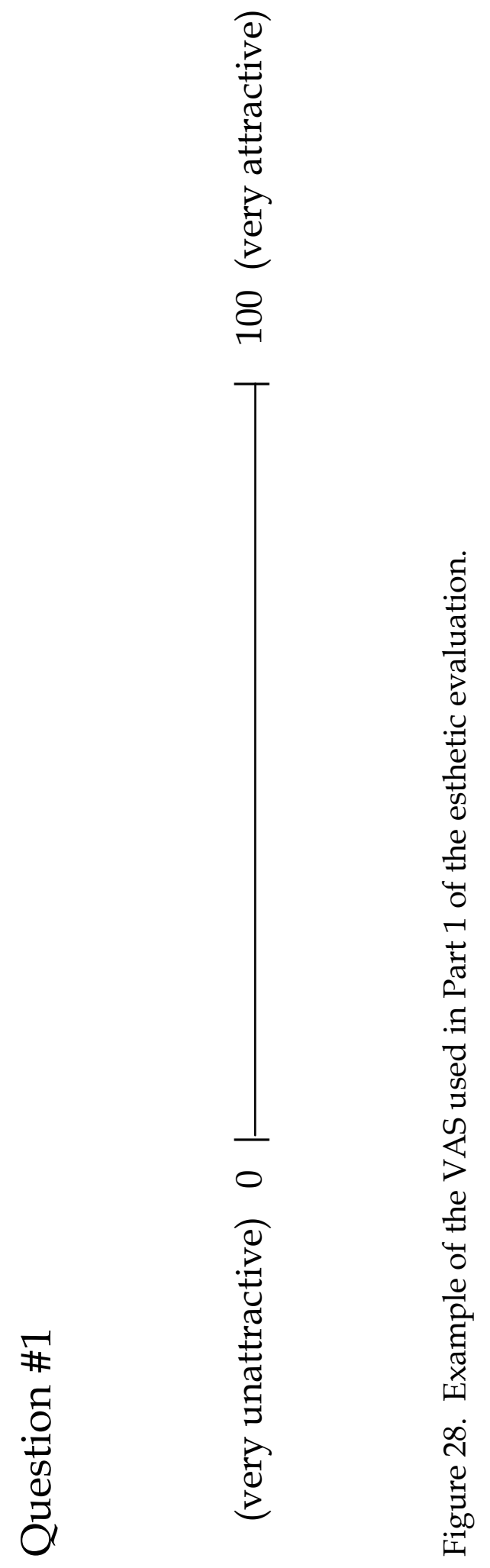




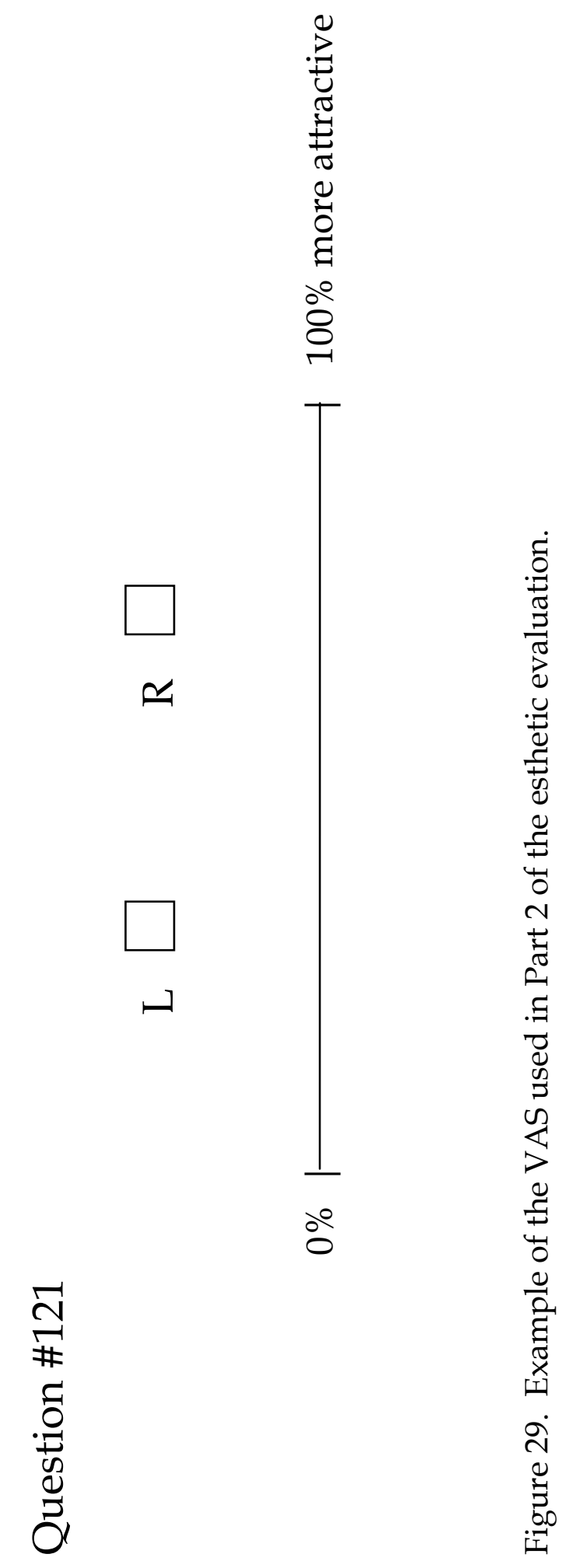


Both the MARA and Edgewise silhouettes were randomly presented on a solid-blue background in a timed slide-show format. All subject's pretreatment and posttreatment silhouettes were individually presented in random order for evaluation. Next, to assess the amount of profile change, the pretreatment and posttreatment silhouettes were shown side-by-side on a single view, also in random order (pretreatment on the left and posttreatment on the right, or vice versa), and observers were asked if they preferred the profile esthetics of the profile presented on the right or on the left by checking a box marked $L$ for left or $R$ for right on the provided handout. Observers then marked the intensity of their preference as described above.

\section{$\underline{\text { Variables }}$}

The integumental and cephalometric landmarks and angles used in the present study are defined below and illustrated in Figures 17, 19, 27, and 30 through 33.

\section{Integumental Landmarks}

Soft Tissue Nasion $\left(\mathrm{Na}^{\prime}\right)$ : The point on the integument at the deepest dorsal concavity below the superciliary arch at the depth of the nasal root (Athanasiou 1995).

Soft Tissue Orbitale (Or'): The soft tissue representation of the lowest (most caudal) point on the inferior rim of the orbit (Athanasiou 1995).

Throat Point (T): The soft tissue point tangent to the cerviomental angle formed by the lower border of the mandible and vertical plane of the neck (Worms et al. 1976; Shelly et al. 2000; Mergen et al. 2004).

Tragion (Tr): The notch just above the tragus of the ear. It lies 1 to $2 \mathrm{~mm}$ below the spina helicis, which is easily palpated (Kolar and Salter 1997).

\section{Cephalometric Landmarks}

Gonion (Go): The most posterior-inferior point on the gonial angle of the mandible. Anatomic Gonion was used, not a mechanical construct (Athanasiou 1995). 


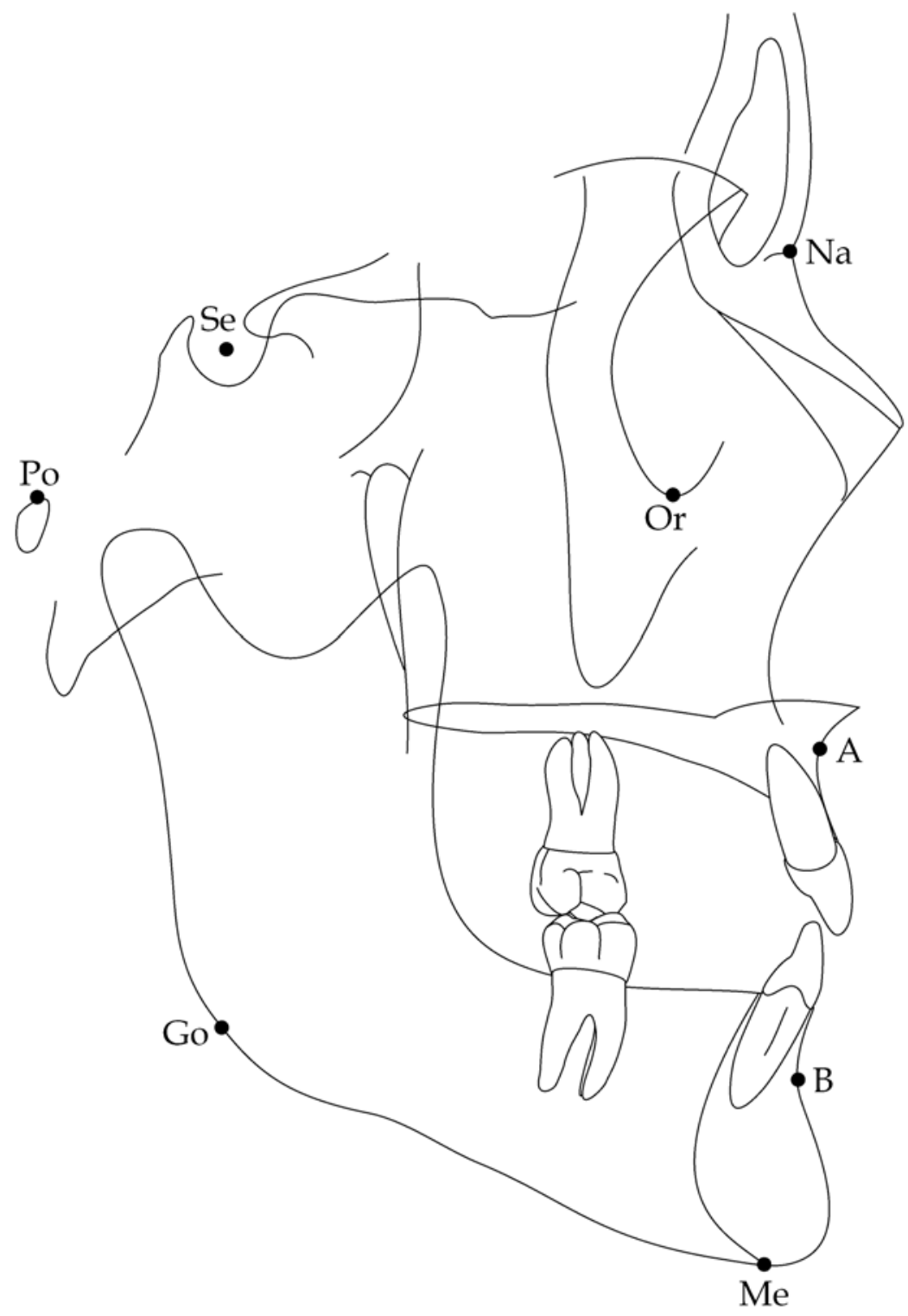

Figure 30. Cephalometric diagram showing locations of the skeletodental landmarks used in this study. 


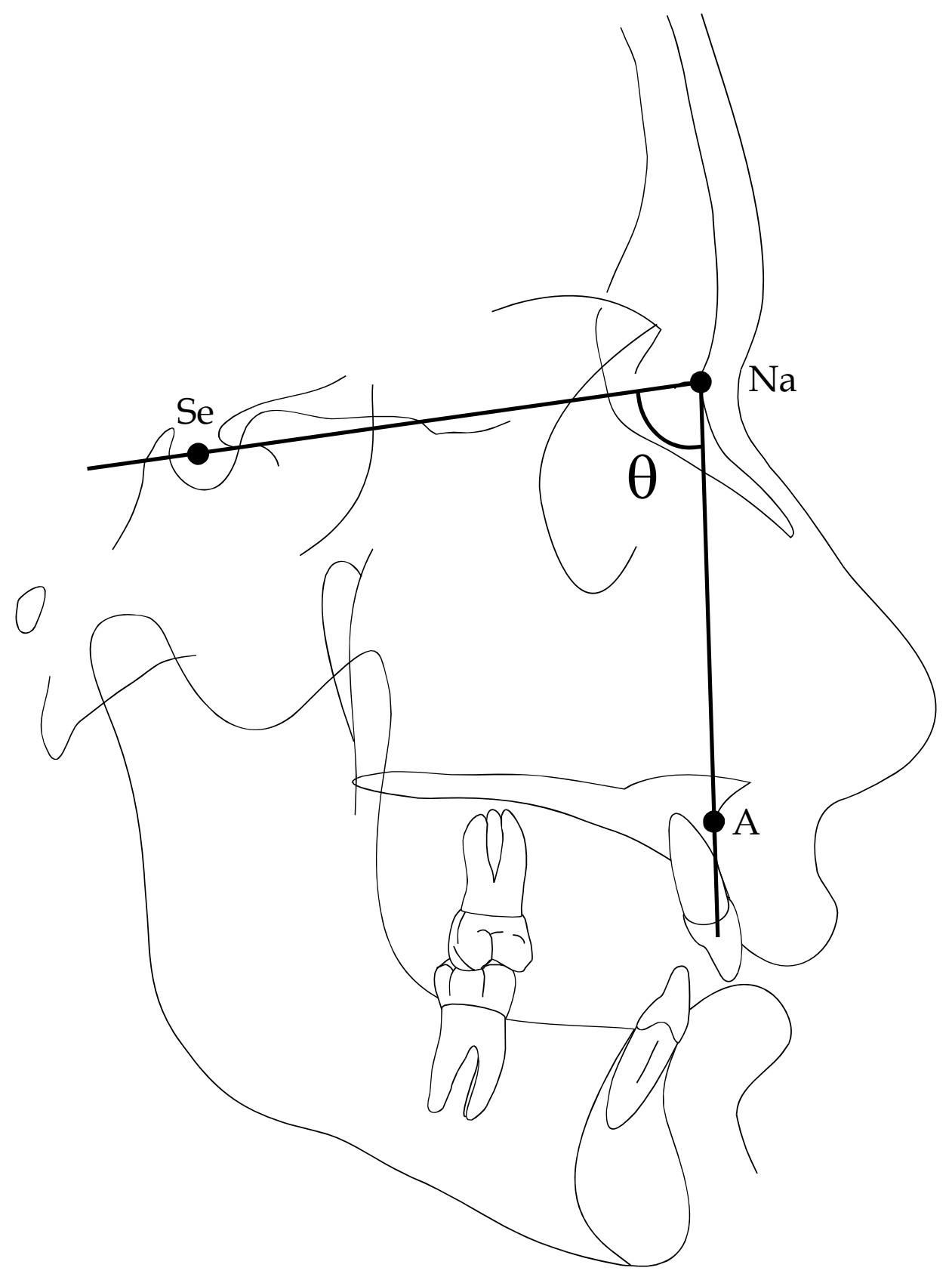

Figure 31. Schematic tracing of a lateral cephalogram showing construction of the SNA angle $(\theta)$. 


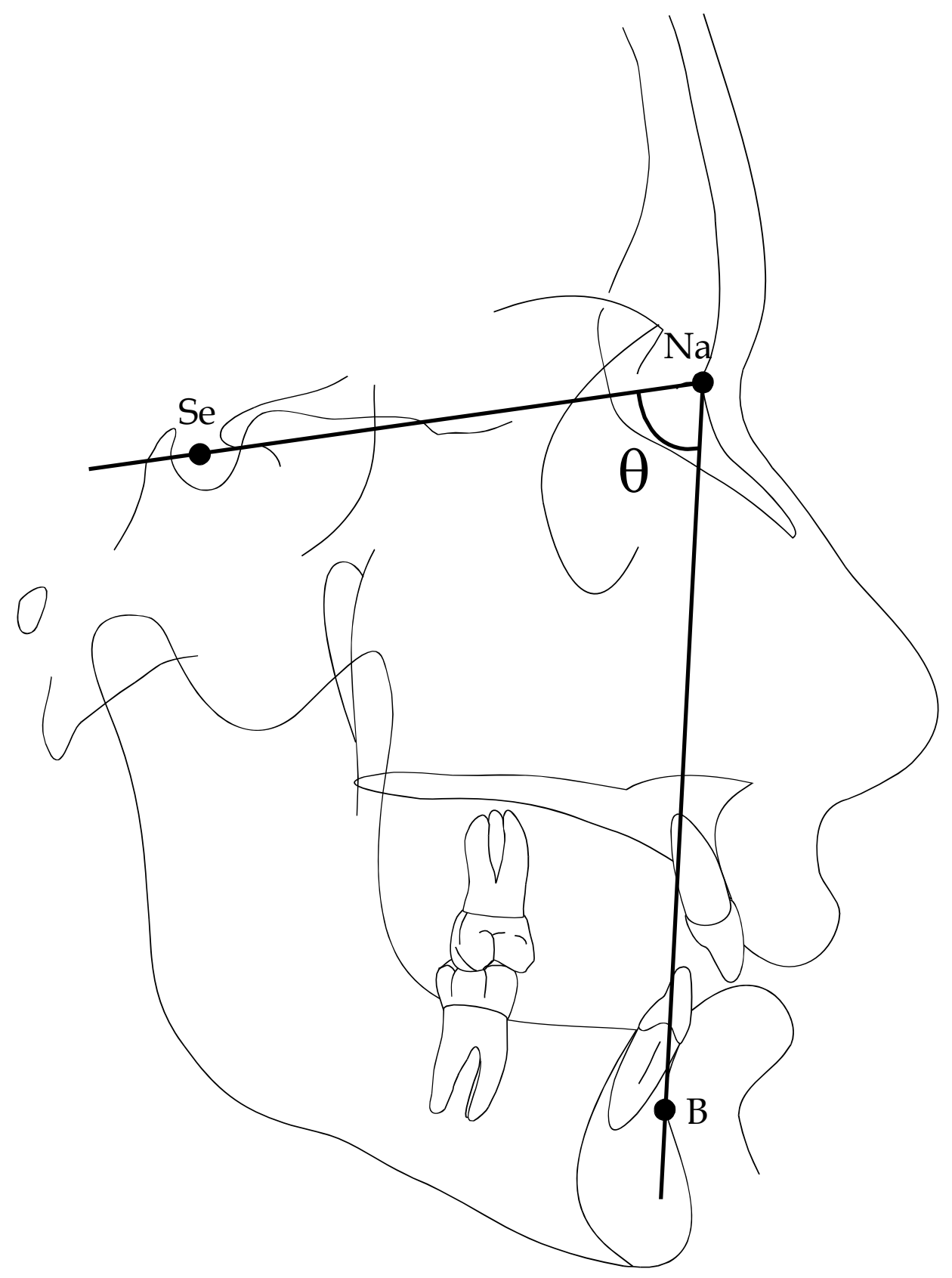

Figure 32: Schematic tracing of a lateral cephalogram showing construction of the SNB angle $(\theta)$. 


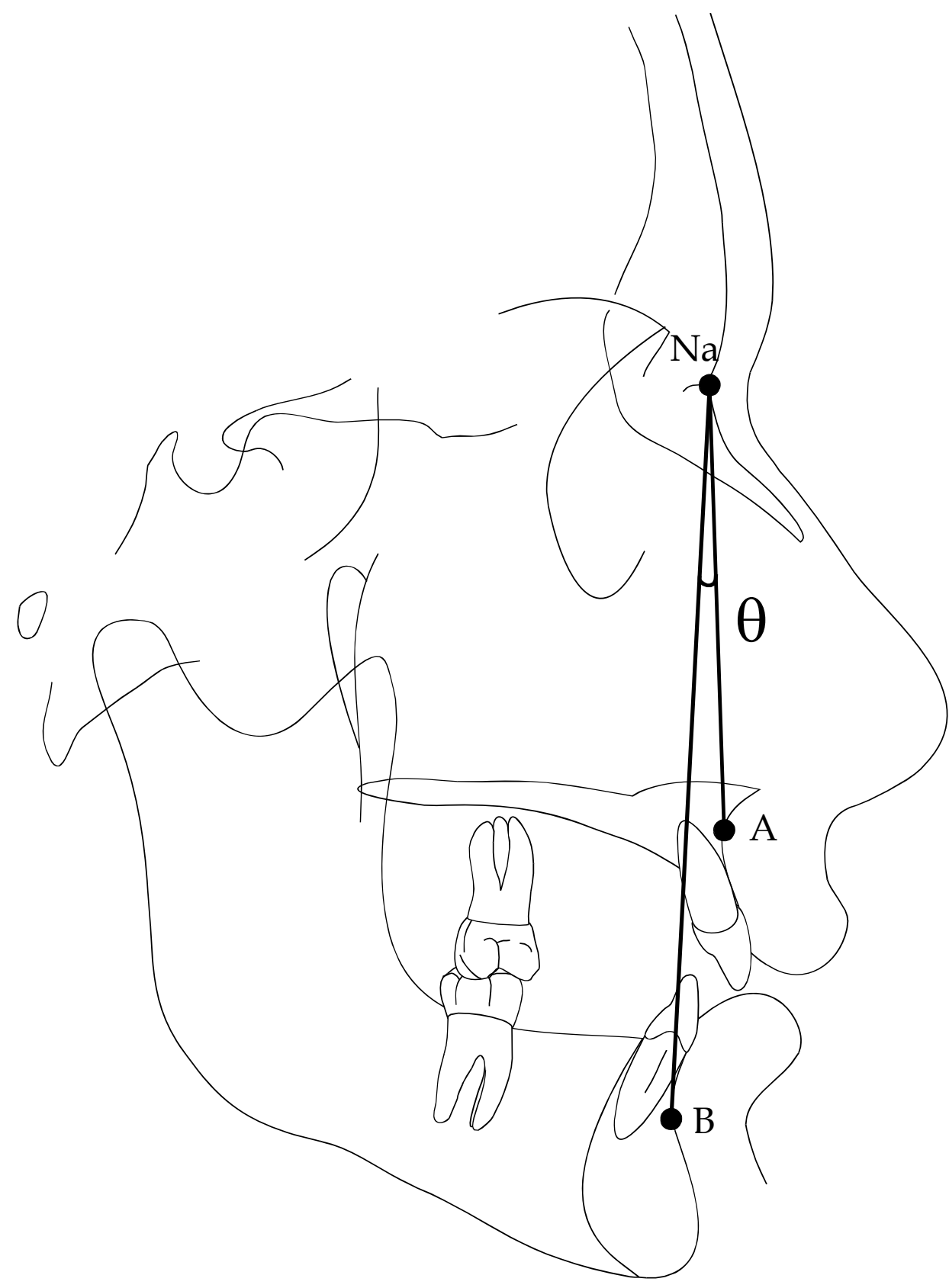

Figure 33. Schematic tracing of a lateral cephalogram showing construction of the angle ANB $(\theta)$. In practice, ANB commonly is evaluated as the difference between SNA and SNB rather than measured directly. 
Menton (Me): The most inferior point onthe exterior symphyseal outline as seen in the lateral film (Athanasiou 1995).

Nasion $(\mathrm{Na})$ : The junction of the frontal and nasal bones at the most dorsal point (Athanasiou 1995).

Orbitale (Or): The lowest (most caudel) point on the inferior margin of the bony orbit (Athanasiou 1995).

Porion (Po): The midpoint of the superior rim of the external auditory meatus. Anatomic Porion was used, not a mechanical construct (Athanasiou 1995).

Sella (Se): Midpoint of the hypophyseal fossa (sella turcica), constructed by inspection (Athanasiou 1995).

Subspinale (Point A): The deepest midline point on the ventral maxillary border inferior to the anterior nasal spine (ANS) and superior to Prosthion (Downs 1948).

Supramentale (Point B): The deepest midline point on the bony curvature of the mandible between Infradentale and Pogonion (Downs 1948).

\section{Cephalometric Angles}

Sella-Nasion-Point A Angle (SNA): The angle formed by the intersection of the two lines formed by Sella-Nasion and by Nasion-Point A (Steiner 1953).

Sella-Nasion-Point B (SNB): The angle formed by the intersection of the lines formed by Sella and Nasion and by Nasion and Point B (Steiner 1953).

Point A-Nasion-Point B (ANB): The angle formed by the intersection of the lines formed by Point A and Nasion and by Nasion and Point B (Steiner1953).

Frankfort-Mandibular plane Angle (FMA): The inferior-anterior angle formed by the intersection of Frankfort horizontal plane (Orbitale-Porion) and the mandibular plane (Gonion-Menton) (Tweed 1969). 


\section{$\underline{\text { Statistical Methods }}$}

Data were collected in a Microsoft Excel spreadsheet (Microsoft, Redmond, WA), then transferred to the statistical package termed JMP 5.0.2 (SAS Institute Inc., Cary, NC). Exploratory data analysis (Tukey 1977) was performed to find outliners possibly caused by technical errors; technical errors (just a very few data entry errors) were then corrected.

Conventional descriptive statistics (e.g., Sokal and Rohlf 1995) were calculated for each treatment group, observation panel, and male and female observers; these (and abbreviations) are sample size (n, taken as counts of individuals), the arithmetic mean $(\overline{\mathrm{x}})$, the median, the standard deviation (sd), and the minimum and maximum values.

Statistical analysis relied on factorial (model 1) analysis of variance (ANOVA). Analytic strategies were those suggested by Winer and coworkers (1991) and Sokal and Rohlf (1995). Independent variables of interest were, in no particular order, (A) sex of the subject, (B) sex of the observer, (C) the observer's group (either graduate student, experienced orthodontist, or lay person), (D) treatment group (MARA or Edgewise alone). In each instance, the full ANOVA models were computed, so all of the interaction effects could be evaluated.

Paired t-tests and one-sample t-tests were used to assess whether the intreatment changes were systematically difference from zero (two tail tests).

The conventional alpha level of 0.05 was used throughout, and all of the tests were two-tail. No correction was made for multiple comparisons.

Salient results of the analysis were graphed using Delta Graph 5.5 (Red Rock Software, Inc., Salt Lake City, UT) on a Macintosh platform. 


\section{CHAPTER IV \\ RESULTS}

\section{Evaluation of the Silhouettes at the Pretreatment Examination}

A three-way factorial ANOVA model was used to examine the effects (and the interactions among) (1) the patient's sex, (2) sex of the observer, and (3) the three groups of observers (either experienced orthodontist, orthodontic graduate student, or lay person). Pointedly, we did not include treatment type in this model because the intent here was to evaluate the "ancillary" variables before addressing the central issue of the project. Results of the full three-way model are in Table 4, where one can see that all three main effects are all highly significant statistically as well as one of the first-order interactions (i.e., observer's sex by group). The nature of this interaction is graphed in Figure 34; male observers, regardless of group, scored the patients' faces as more esthetic (higher VAS score) than did the female observers. However, the difference between the male and female graduate students (residents) is several-fold larger than in the other two groups of observers, and this is the source of the significant interaction effect.

These several differences are evident in the bar chart of the VAS scores of the pretreatment silhouettes (Figure 35): One, of the three groups the experienced orthodontists rated the silhouettes more favorably (higher median VAS scores). Two, within each of the three groups of observers, men evaluated the silhouettes more favorably than their female counterparts; this sex difference was greatest between the male and female graduate students. Three, there is considerable variation ("differences of opinions") within and among each of these six groups of observers.

It is not apparent why the residents are so 'polarized' in their profile assessments based on their sex. All that is evident from the analysis (Figure 34) is that female residents scored the patients' profiles more 'harshly' than their male counterparts. Since, on the other hand, patient sex by observer sex interaction is not significant $(\mathrm{P}=0.82)$, the female residents were indifferent to the patients' sex (which was unknown to them); they systematically scored all of the profiles comparatively low.

Absence of any systematic difference between the two treatment groups at the start of treatment is evident from the boxplots in Figure 36. The medians are virtually identical, and there is considerable overlap of the two distributions. 
Table 4. Results of ANOVA tests of the pretreatment VAS scores. ${ }^{1}$

\begin{tabular}{lrrrr}
\hline \multicolumn{1}{c}{ Source } & df & SSQ & F Ratio & P Value \\
\hline Patient Sex & & & & \\
Observer Sex & 1 & 4844.21 & 14.28 & 0.0002 \\
Observer Group & 1 & 5161.23 & 15.22 & $<0.0001$ \\
Pt Sex-x-Obs Sex & 2 & 17797.73 & 26.24 & $<0.0001$ \\
Pt Sex-x-Group & 1 & 17.53 & 0.05 & 0.8202 \\
Obs Sex-x-Group & 2 & 1028.29 & 1.52 & 0.2198 \\
Pt Sex-x-Obs Sex-x-Group & 2 & 6489.63 & 9.57 & $<0.0001$ \\
& 2 & 61.16 & 0.09 & 0.9138 \\
\hline
\end{tabular}

${ }^{1}$ Abbreviations are patient $(\mathrm{Pt})$, and observer $(\mathrm{Obs})$. 


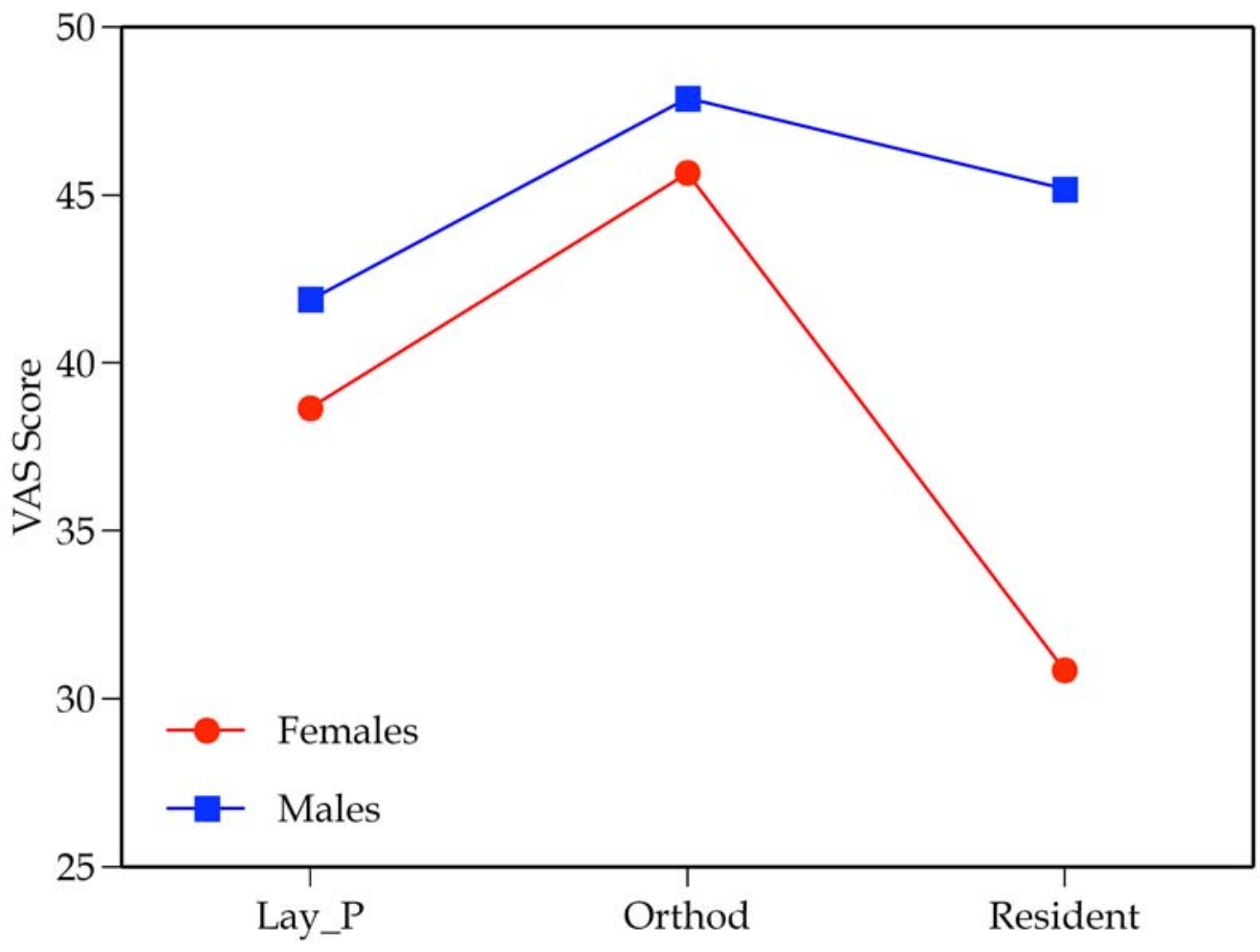

Figure 34. Mean VAS scores, by sex and group of observers, at the pretreatment examination. 


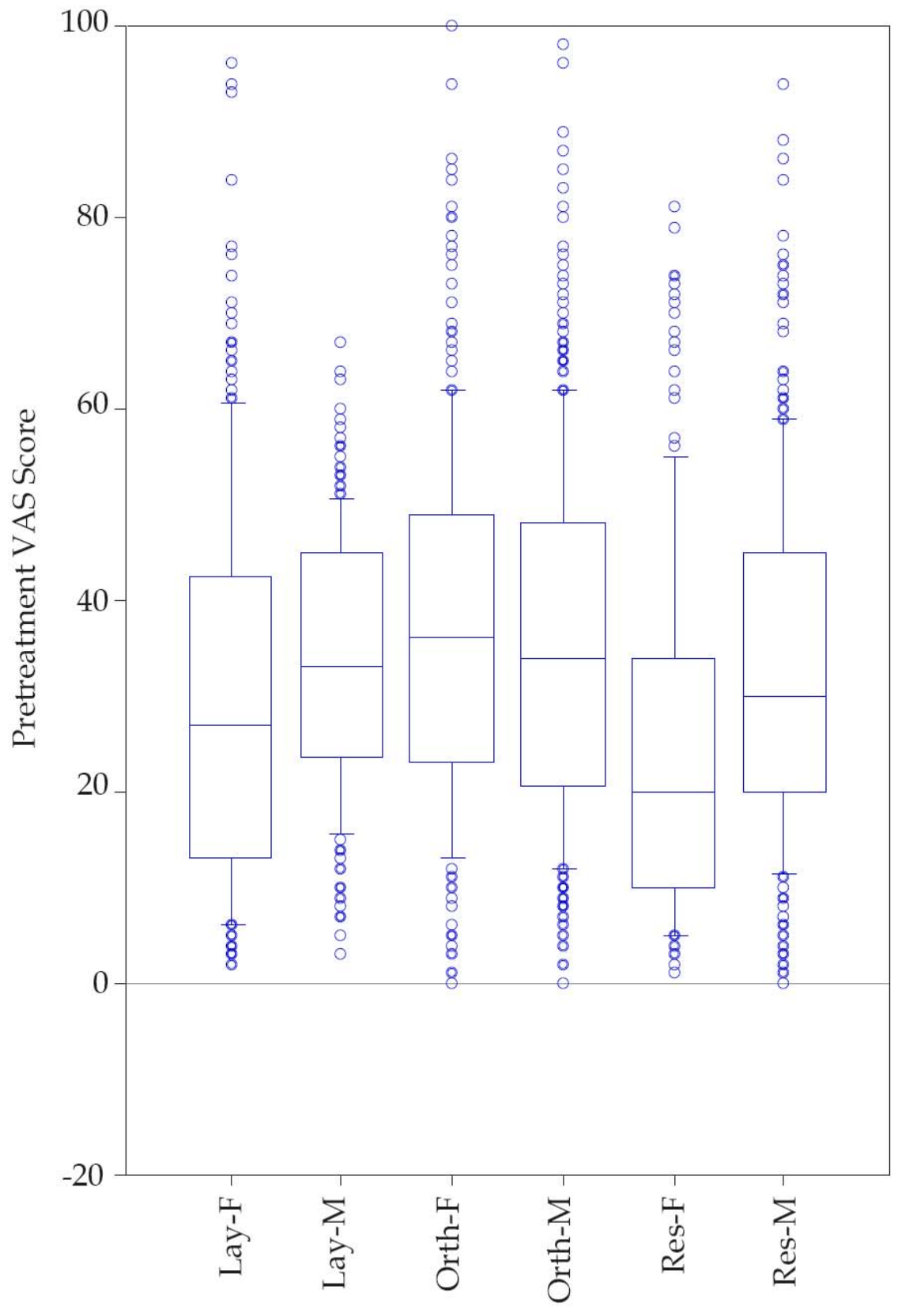

Figure 35. Bar charts of the VAS scores at the start of treatment, by rater group and sex (i.e., sex of the rater). The interesting (and statistically significant) features here is that female raters (coded $\mathrm{F}$ ) tend to assign lower (less esthetic) VAS scores than male raters (coded M). 


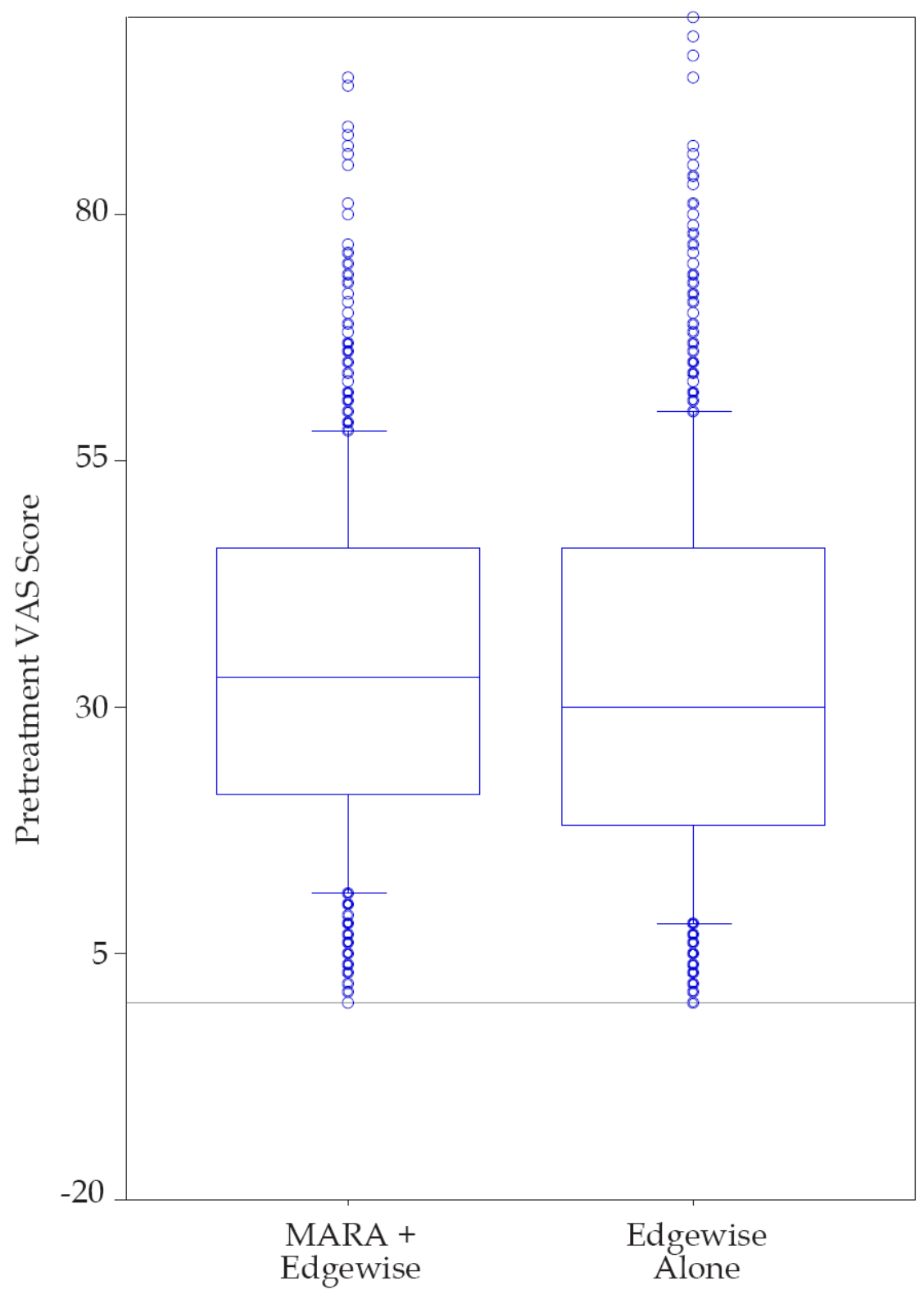

Figure 36. Bar charts of the pretreatment VAS scores, by treatment group. The appreciable overlap and the equivalence of median VAS scores argue for the comparability of the starting conditions in the two groups. 
Strictly, a significant interaction effect means that the main effects are biased and the analyses should be performed separately, in this case by sex of the observer. In fact this was done, but it did not alter the results, and we interpret that output shown in Table 4. VAS scores were significantly different based on the sex of the patient; female profiles were judged to be more esthetic (higher VAS score) than boys (means of 34 and 31, respectively). Sex of the observer also made a significant difference, as discussed above, because male judges scored the patients' profiles as more esthetic (higher VAS score) than female judges (means of 34 and 31, respectively). The results for the third main effect are evident in Figure 34, where the groups of observers differed: Residents were the most critical $(\bar{X}=29)$, followed by the sample of lay people $(\bar{X}=32)$, and then the experienced orthodontists $(\bar{X}=36)$.

\section{Evaluation of the Silhouettes at the Posttreatment Examination}

Statistical analysis of the profile assessments at the posttreatment examination were virtually identical to those just described for the start of treatment (Table 5). Again, mode of treatment is not considered here because it is practical to examine the other sources of variation (and their influences on the VAS scores) first. As above, all three main effects are highly significant statistically and so is the observers' sex by group interaction. Figure 37 shows the source of the significant interaction; again, the sample of female graduate students supplied low VAS scores vis-à-vis their male counterparts. Here too, the sample of experienced orthodontists scored the posttreatment profiles virtually identically regardless of their (the orthodontists') sex. (While the mean VAS score for females is just above that for males in Figure 37, the sex difference is far from significant.) In fact, then, Figure 37 discloses a separate level of intersex preference for each of the three groups of observers: (1) Males in the lay sample 'liked' the profile somewhat more than the lay female observers. (2) There was no difference in VAS score by sex of the orthodontist. (3) Female graduate students judged the profiles much 'harsher' than male students based on the VAS scores awarded.

Alternatively, while these sex differences are of interest in passing, they actually just reflect a sex difference in the range of VAS scores given. Some observers used the whole $10 \mathrm{~cm}$ scale, but the females, especially the female graduate students, tended to cluster their responses nearer the low end of the scale. 
Table 5. Results of ANOVA tests of the posttreatment VAS scores.

\begin{tabular}{lrrrr}
\hline \multicolumn{1}{c}{ Source } & df & \multicolumn{1}{c}{ SSQ } & F Ratio & P Value \\
\hline Patient Sex & & & & \\
Observer Sex & 1 & 5624.94 & 12.87 & 0.0003 \\
Observer Group & 1 & 19954.26 & 45.64 & $<0.0001$ \\
Pt Sex-x-Obs Sex & 2 & 27681.77 & 31.66 & $<0.0001$ \\
Pt Sex-x-Group & 1 & 19.38 & 0.04 & 0.8333 \\
Obs Sex-x-Group & 2 & 176.07 & 0.20 & 0.8176 \\
Pt Sex-x-Obs Sex-x-Group & 2 & 12704.02 & 14.53 & $<0.0001$ \\
& 2 & 208.52 & 0.24 & 0.7879 \\
\hline
\end{tabular}




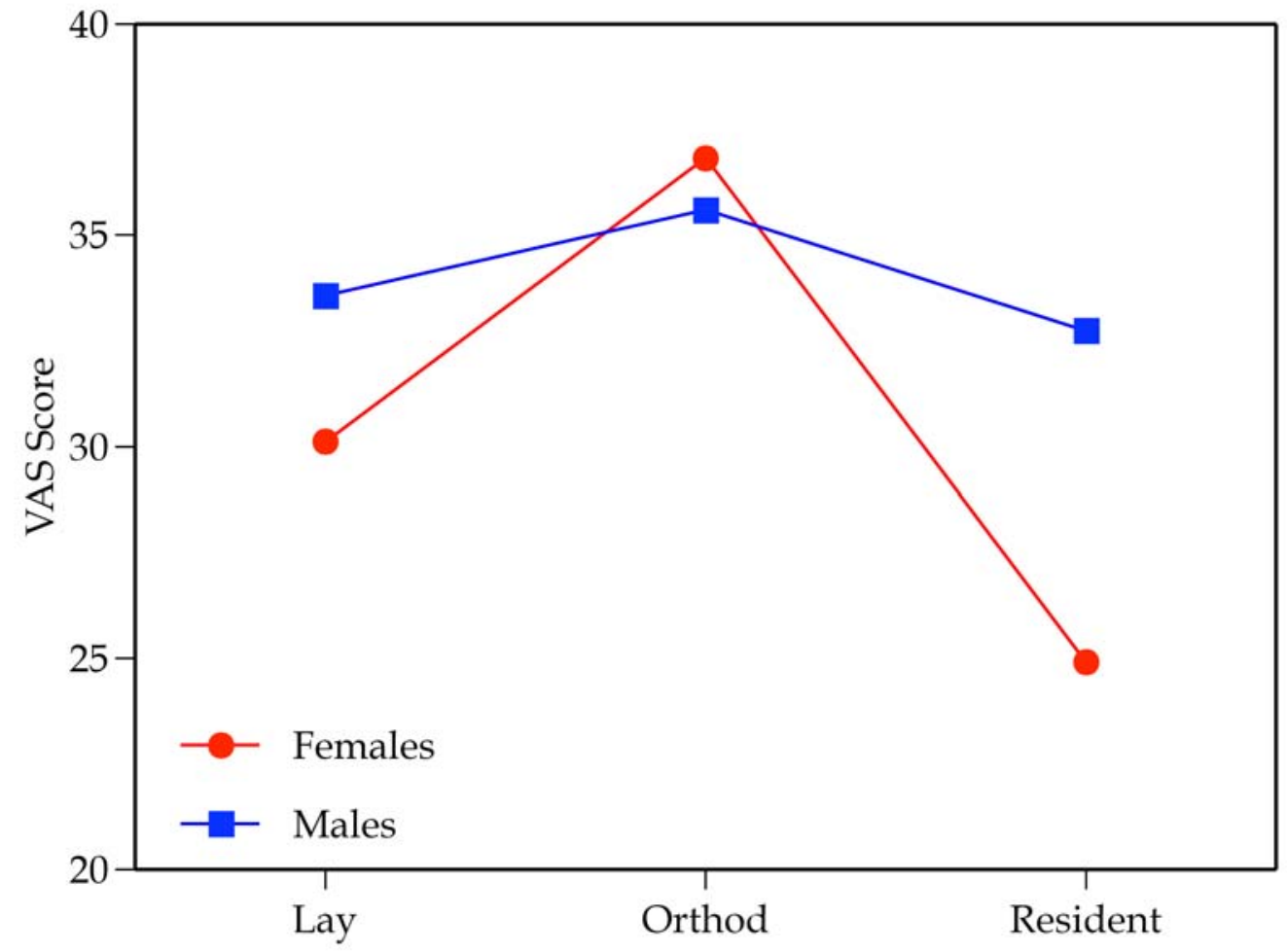

Figure 37. Mean VAS scores, by sex and group of observers, at the posttreatment examination. 


\section{$\underline{\text { Treatment Effects Evaluated at Posttreatment }}$}

If there is a perceptual difference between patients treated with Edgewise mechanics with or without the MARA appliance, one simple test is to compare the observers' VAS scores between the two treatments at the end of orthodontic treatment. This is not a sensitive test but we explored it here because the data are easy to analyze. The next section provides a more definitive test of the treatment changes.

Table 6 shows the results of ANOVA testing for a difference in profile esthetics between treatments while accounting for the three groups of observers and sex of the observers. Just as detailed in the prior section, however, there is a highly significant first-order interaction brought about because male raters tend to score the profiles higher. We therefore separated the data of male and female observers and analyzed them individually so the confounding interaction is avoided. In passing, the nature of the sex differences between observers' scores is shown in Figure 38. These are box plots for the VAS scores given by each of the three groups. The median score is higher for male observers than female observers in the sample of lay people. This same sex difference is evident among the sample of orthodontists, and the sex difference (male > female) is greatest among the sample of graduate students, where female graduate students rated the posttreatment silhouettes most harshly (median VAS about 28).

Table 7 lists the results of the two-way ANOVA for female observers alone, and Table 8 lists results for male observers alone. Results are concordant between these tables: treatment type is not discernibly different statistically.

Tables 7 and 8 do show highly significant differences in the VAS scores depending who the observers were, and this is shown in Figure 39. Male observers tend to score all of the profiles as more esthetic (higher VAS scores) than female raters. Experienced orthodontists scored these posttreatment profiles higher than orthodontic residents or lay people.

\section{Treatment Changes}

\section{Comparing Pretreatment and Posttreatment VAS Scores}

Orthodontic treatment is intended to improve esthetics and function of the occlusion, and it also holds the promise of enhancing facial esthetics (McNamara and Brudon 1993). One aspect of these treatment expectations-at least on the 
Table 6. Results of three-way ANOVA evaluating posttreatment VAS scores, with observer sex in the model.

\begin{tabular}{llrrr}
\hline \multicolumn{1}{c}{ Source } & df & \multicolumn{1}{c}{ SSQ } & F Ratio & P Value \\
\hline TX Type & 1 & 1341.65 & 3.05 & 0.0808 \\
Group & 2 & 27681.77 & 31.49 & $<0.0001$ \\
Obs Sex & 1 & 19954.26 & 45.40 & $<0.0001$ \\
TX Type-x-Group & 2 & 153.08 & 0.17 & 0.8402 \\
TX Type-x-Obs Sex & 1 & 18.94 & 0.04 & 0.8356 \\
Group-x-Obs Sex & 2 & 12704.02 & 14.45 & $<0.0001$ \\
TX Type-x-Group-x-Obs Sex & 2 & 487.55 & 0.55 & 0.5743 \\
\hline
\end{tabular}




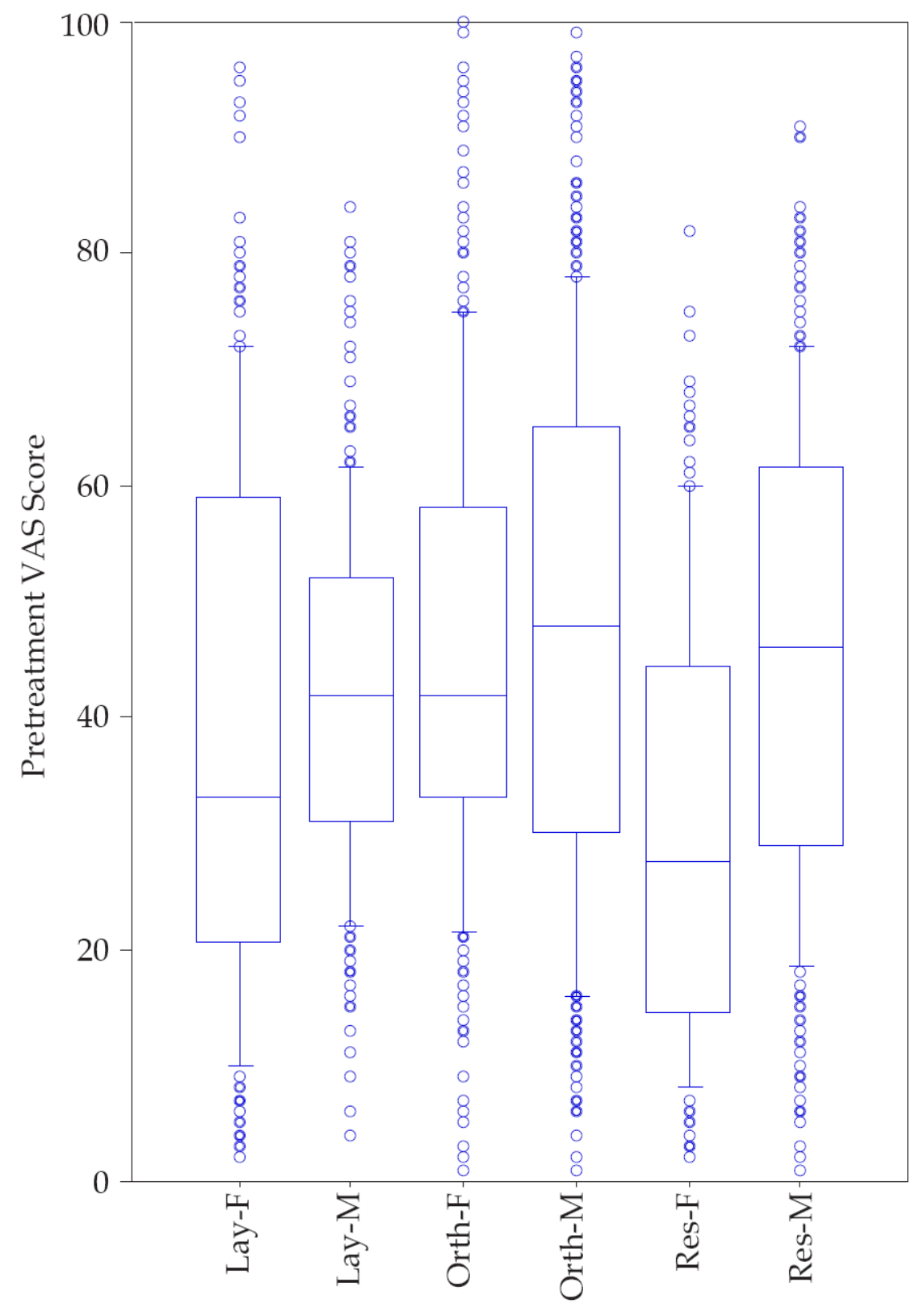

Figure 38. Bar charts of the VAS scores at the end of treatment, by rater group and sex (i.e., sex of the rater). The statistically significant feature here is that female raters tend to assign lower (less esthetic) VAS scores than male raters; this sex difference occurs in all three groups of observers. 
Table 7. Results of two-way ANOVA evaluating posttreatment VAS scores among female observers alone.

\begin{tabular}{lcrrr}
\hline \multicolumn{1}{c}{ Source } & df & \multicolumn{1}{c}{ SSQ } & F Ratio & P Value \\
\hline TX Type & & & & \\
Group & 1 & 673.41 & 1.48 & 0.2248 \\
TX Type-x-Group & 2 & 25047.54 & 27.45 & $<0.0001$ \\
\hline
\end{tabular}


Table 8. Results of two-way ANOVA evaluating posttreatment VAS scores among male observers alone.

\begin{tabular}{lrrrr}
\hline \multicolumn{1}{c}{ Source } & df & \multicolumn{1}{c}{ SSQ } & F Ratio & P Value \\
\hline TX Type & 1 & 691.71 & 1.61 & 0.2047 \\
Group & 2 & 7326.95 & 8.53 & 0.0002 \\
TX Type-x-Group & 2 & 89.96 & 0.10 & 0.9006 \\
\hline
\end{tabular}




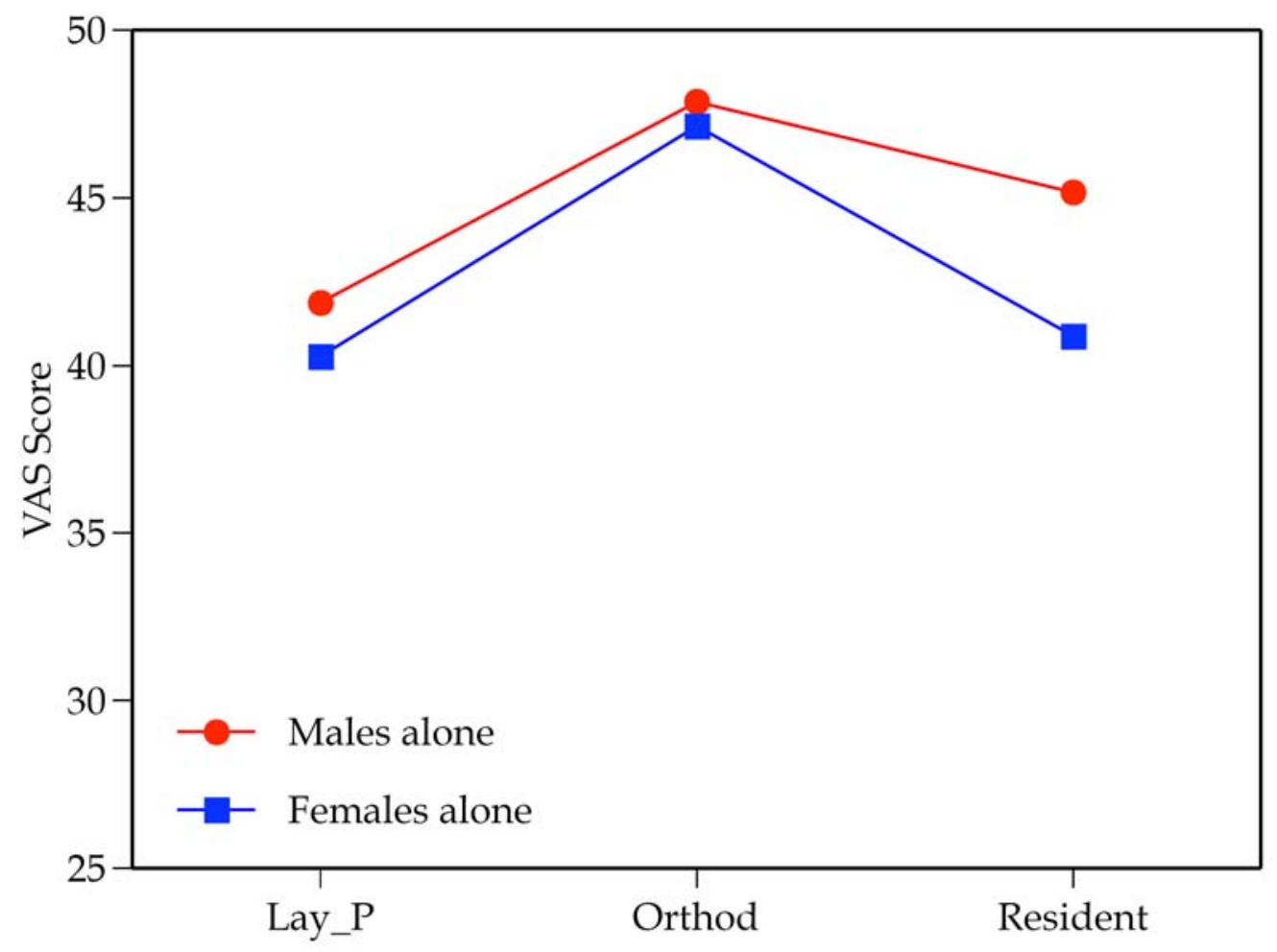

Figure 39. Mean VAS scores at posttreatment, by group of observers, depending on whether the observers were males or females. 
part of the public-is that the facial profile should improve over the course of treatment. This certainly was seen in the present study as judged by the increase in VAS scores from pre- to posttreatment. Average VAS scores increased from 32 to 42 in the Edgewise sample, an increase of 10 points, which is highly significant by paired $t$-test $(\mathrm{t}=15.3 ; \mathrm{df}=1,049 ; \mathrm{P}<0.0001)$. Improvement is virtually identical in the MARA sample, from 34 to 44 . The change of 10 points also is highly significant statistically $(\mathrm{t}=16.6 ; \mathrm{df}=1,049 ; \mathrm{P}<0.0001)$.

From these statistics, it may be evident that the improvements are essentially identical between the two treatments. Absence of a difference between the three groups of observers is shown in Figure 40, where the median improvements in each group of observers is about 30 VAS points, but there is almost complete overlap of the ranges of the scores. This is shown more formally in Tables 9 and 10. As before, a significant interaction effect was avoided by analyzing data from the male and female observers separately. In neither case (Tables 9,10$)$ is there any suggestion that the extent of the esthetic improvement differed between the two treatments. That is, (1) VAS scores increased significantly in each treatment modality, but (2) since the starting (pretreatment) scores were the same on the average, (3) the improvements during treatment were indistinguishable statistically.

\section{Observers' Perceptions of Pretreatment and Posttreatment Profiles}

Orthodontic treatment does not invariably improve esthetics of the facial profile, and Bowman and Johnston (2000) devised a method of evaluating the perceived changes during treatment. We parallel that method here (see Methods). In brief, the pair of pre- and posttreatment silhouettes were shown together but in random order. The rater first marked which profile he preferred, and then the rater used the VAS scale (100 $\mathrm{mm}$ in length) to score how much he preferred one silhouette over the other. The data subsequently were coded (1) to denote whether the silhouette improved (a positive VAS score) or (2) worsened (a negative score) during treatment. We were struck by the magnitude and dispersion of the VAS changes (Figure 41). A third of the changes (35\%) were negative, meaning that esthetics of the profile degraded during treatment. This graph also shows that some profile changes elicited very strong reactions among the raters. Hardly any treatment changes $(0.17 \%)$ were scored as a VAS change between -80 and $100 \mathrm{~mm}$. Overall, most changes $(65 \%)$ were judged to be improvements in the profile. And some changes (1.6\%) involved dramatic improvements of +60 to $+100 \mathrm{~mm}$. It cannot be determined from these data how much of any change (or how many changes) are attributable to orthodontic treatment, which normally is focused on lip postures (King 1960). Evaluation of facial silhouettes involves nose, lip, chin, and neck relationships-much of which 


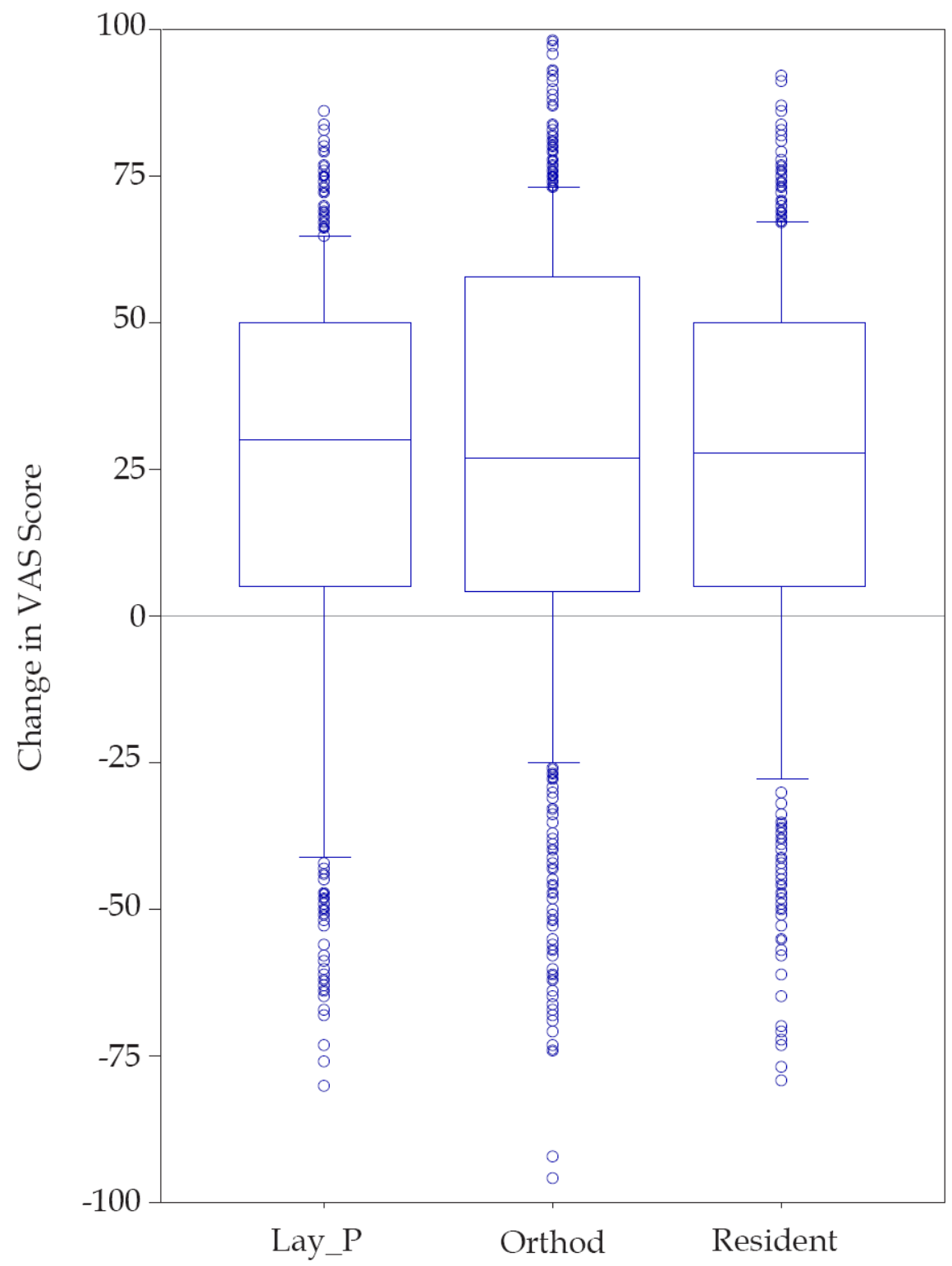

Figure 40. Bar charts of the change in VAS scores, by background of the observer. As verified statistically, none of the three groups of raters differed in its perception of the change in facial profiles during treatment. 
Table 9. Evaluation of the changes in VAS scores (male observers alone).

\begin{tabular}{lcrcc}
\hline \multicolumn{1}{c}{ Source } & df & \multicolumn{1}{c}{ SSQ } & F Ratio & P Value \\
\hline TX Type & 1 & & & \\
Pt Sex & 1 & 129.66 & 0.07 & 0.7900 \\
Group & 2 & 3776.31 & 0.31 & 0.5773 \\
TX Type-x-Pt Sex & 1 & 1361.67 & 4.52 & 0.0111 \\
TX Type-x-Group & 2 & 158.83 & 0.16 & 0.0714 \\
Pt Sex-x-Group & 2 & 382.29 & 0.46 & 0.8270 \\
TX Type-x-Pt Sex-x-Group & 2 & 355.73 & 0.43 & 0.6532 \\
\hline
\end{tabular}


Table 10. Evaluation of the changes in VAS scores (female observers alone).

\begin{tabular}{lcrcc}
\hline \multicolumn{1}{c}{ Source } & df & SSQ & F Ratio & P Value \\
\hline TX Type & 1 & 476.96 & 1.10 & 0.2956 \\
Pt Sex & 1 & 4.08 & 0.01 & 0.9229 \\
Group & 2 & 1060.43 & 1.22 & 0.2965 \\
TX Type-x-Pt Sex & 1 & 385.27 & 0.88 & 0.3472 \\
TX Type-x-Group & 2 & 1666.63 & 1.91 & 0.1482 \\
Pt Sex-x-Group & 2 & 802.23 & 0.92 & 0.3985 \\
TX Type-x-Pt Sex-x-Group & 2 & 584.53 & 0.67 & 0.5114 \\
\hline
\end{tabular}




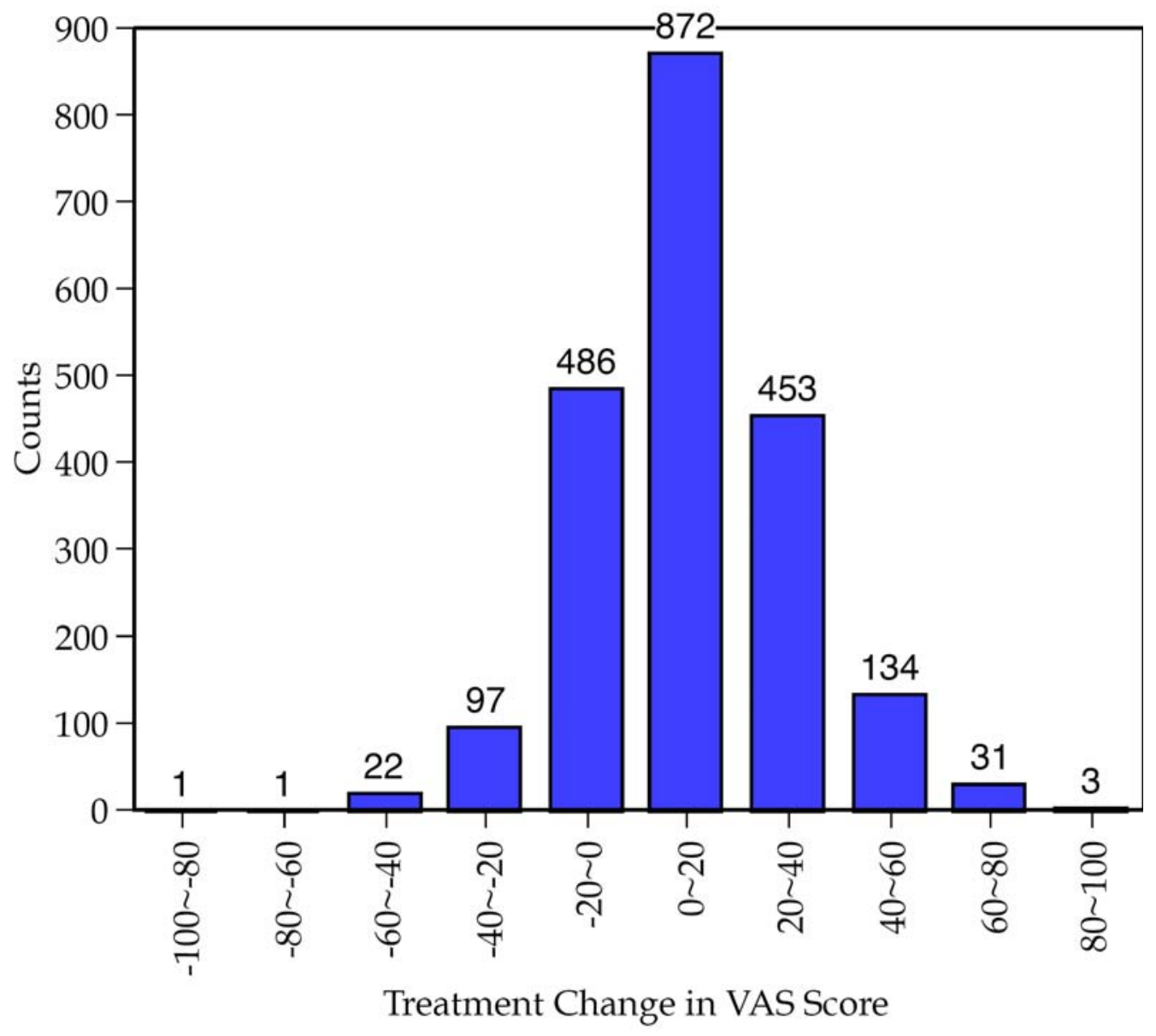

Figure 41. Distribution of in-treatment changes in VAS scores. Negative values indicate that the profile became less esthetic during treatment; positive changes indicate that it improved. 
is outside the orthodontist's purview. A four-way factorial ANOVA was used to analyze these data (Table 11).

One interaction term is significant $(\mathrm{P}=0.01)$-which is the same effect seen previously, namely that male raters tend to assign higher scores than females. Treatment type itself is far from significant $(\mathrm{P}=0.60)$, indicating that Edgewise treatment with or without the MARA appliance produces the same integumental profile as evaluated esthetically. Absence of a discernible treatment difference also is shown in Figure 42; medians are virtually identical and there is almost complete overlap of the two distributions of VAS scores. Sex of the patient does influence the outcome $(\mathrm{P}=0.006)$ because the improvement was judged to be more-often positive in girls. This sex difference is graphed in Figure 43, where female orthodontic patients were given higher VAS scores by observers of both sexes. The one other significant effect is due to the sex of the observer where the tendency (noted previously) for female raters to judge the profiles more harshly (i.e., lower VAS scores) is evident. The higher VAS scores given by male raters versus female raters is graphed in Figure 44, which shows that the median is higher when males scored the profiles than when women scored the same profile.

As we have noted, there was considerable variation among observers' VAS scores. Still, it is of interest to identify which profiles were judged to change the most during the course of treatment. A one-way ANOVA was used to test for the VAS change among all orthodontic subjects (with all observers' scores contributing to the perceived change). The three subjects evaluated as experiencing the greatest improvement during treatment were (1) MARA Subject \#9, a male treated with Edgewise mechanics and a MARA (Figure A9) having a mean change of +29 , (2) Matched Subject \#7, a female treated with Edgewise mechanics alone (Figure A37) having a mean change of +30 , and (3) Matched Subject \#13, a boy treated with Edgewise mechanics alone (Figure A43) having a mean change of +32 .

On the down-side, the three subjects with the most unfavorable changes during treatment were (1) MARA subject \#3, a male treated with Edgewise mechanics and a MARA (Figure A3) having a mean change of -8, (2) Matched subject \#9, a male treated with Edgewise mechanics alone (Figure A39) having a mean change of -16, and (3) Matched subject \#23, a male treated with Edgewise mechanics alone (Figure A53) having a mean change of -19. Of note, (1) the amounts of improvement judged by the VAS scores are greater than the negative changes in these extreme examples, (2) the changes are independent of the subjects' sex (as confirmed earlier by the full analysis), and (3) the changes are not tied to either treatment modality. This last point reinforces our earlier claim 
Table 11. Results of four-way ANOVA evaluating the signed VAS changes during treatment.

\begin{tabular}{lrrrr}
\hline \multicolumn{1}{c}{ Source } & df & \multicolumn{1}{c}{ SSQ } & F Ratio & P Value \\
\hline TX Type & 1 & 377.36 & 0.28 & 0.5981 \\
Pt Sex & 1 & 10444.98 & 7.69 & 0.0056 \\
Group & 2 & 5997.92 & 2.21 & 0.1101 \\
Obs Sex & 1 & 7739.16 & 5.70 & 0.0170 \\
TX Type-x-Pt Sex & 1 & 467.39 & 0.34 & 0.5574 \\
TX Type-x-Group & 2 & 604.32 & 0.22 & 0.8005 \\
TX Type-x-Obs Sex & 1 & 1081.63 & 0.80 & 0.3722 \\
Pt Sex-x-Group & 2 & 883.82 & 0.33 & 0.7222 \\
Pt Sex-x-Obs Sex & 1 & 558.23 & 0.41 & 0.5214 \\
Group-x-Obs Sex & 2 & 11572.78 & 4.26 & 0.0142 \\
TX Type-x-Pt Sex-x-Group & 2 & 3718.81 & 1.37 & 0.2544 \\
TX Type-x-Pt Sex-x-Obs Sex & 1 & 727.91 & 0.54 & 0.4641 \\
TX Type-x-Group-x-Obs Sex & 2 & 1453.41 & 0.54 & 0.5856 \\
Pt Sex-x-Group-x-Obs Sex & 2 & 561.23 & 0.21 & 0.8133 \\
TX Type-x-Pt Sex-x- & & & & \\
Group-x-Obs Sex & 2 & 999.71 & 0.37 & 0.6920 \\
\hline
\end{tabular}




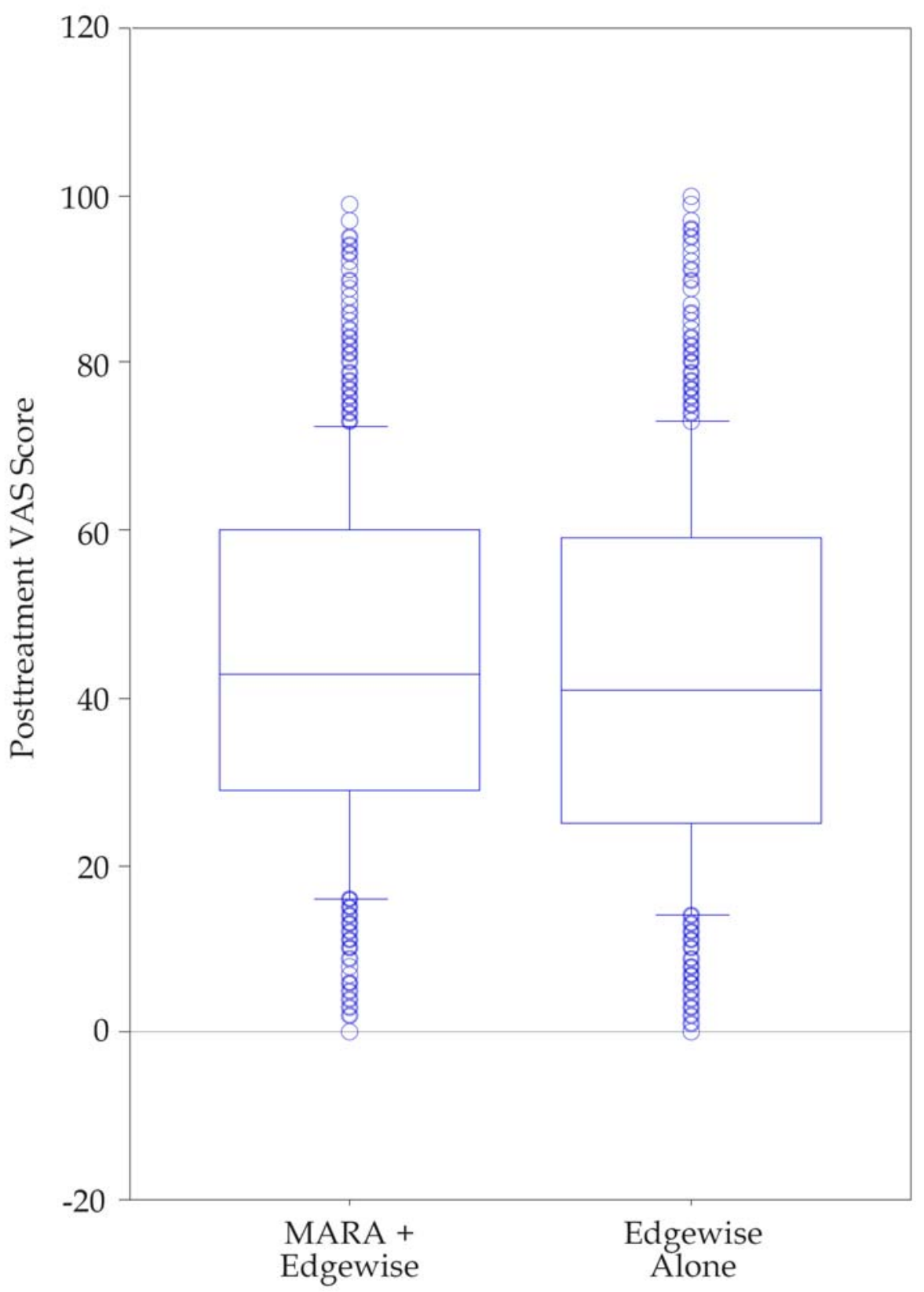

Figure 42. Bar charts of the VAS scores, by treatment group, at the posttreatment examination. The appreciable overlap and the equivalence of median VAS scores argues for the absence of a systematic difference between the two groups. 


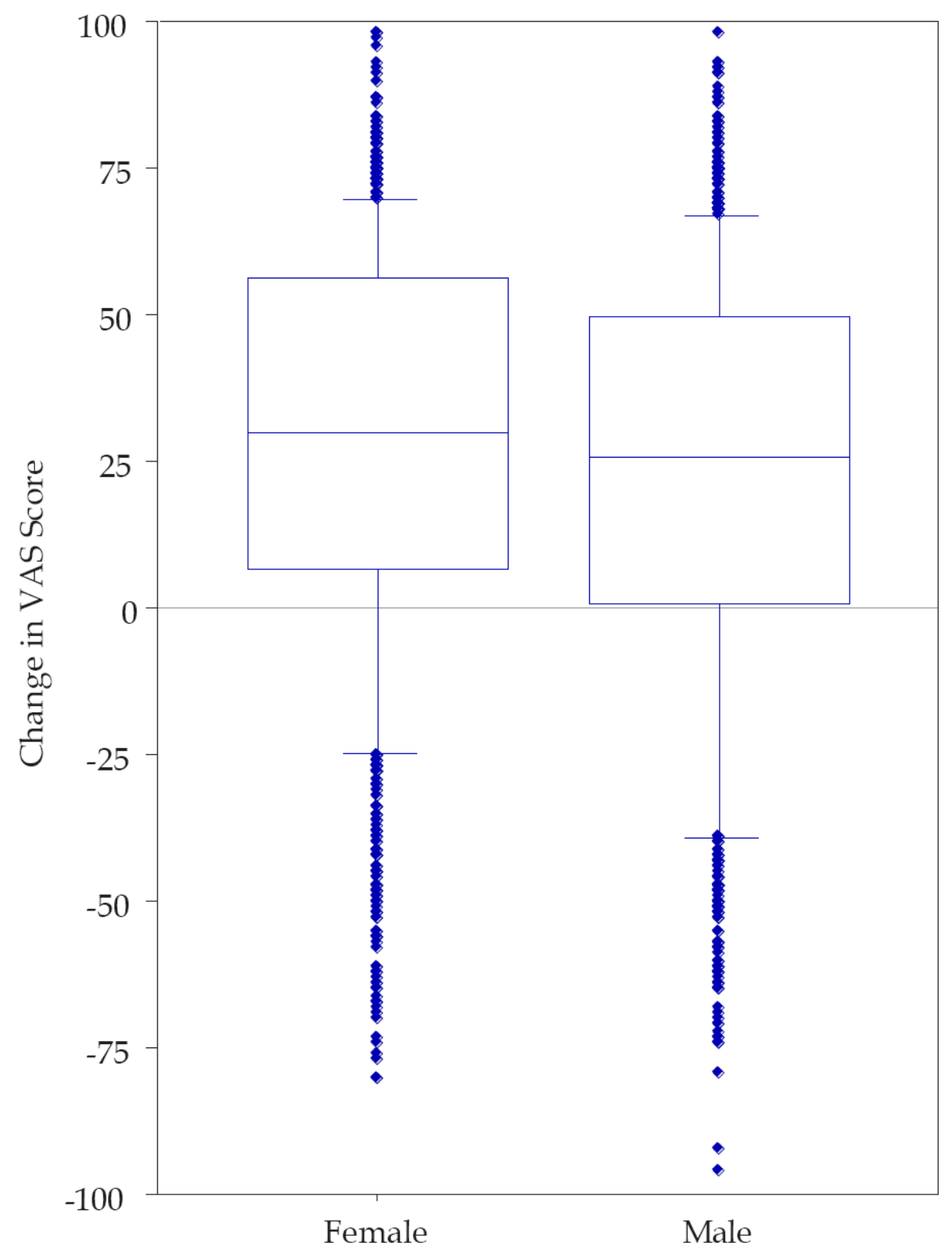

Figure 43. Bar charts of the change in VAS scores, by sex of the patient, as perceived by the raters. Statistically, there is a slightly greater improvement in the females' profiles, but as shown, the inter-rater variability is considerable. 


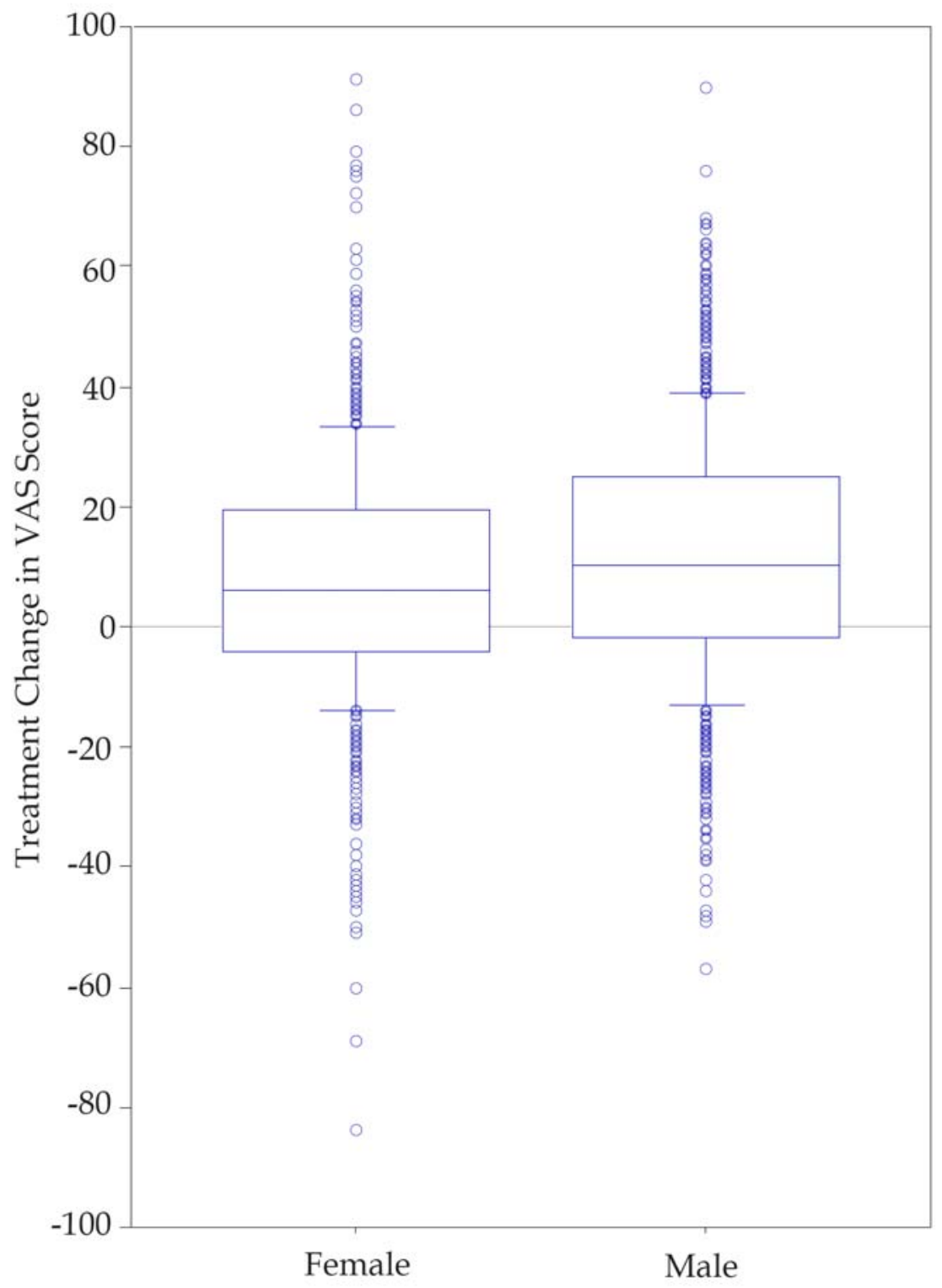

Figure 44. Bar charts of the in-treatment change in VAS scores, partitioned by sex of the observer. The difference is highly significant statistically because male observers judged the changes in facial profiles more favorably, awarding the changes higher VAS scores than the female observers. 
that most changes in the facial profile occur outside what can be modified orthodontically. 


\section{CHAPTER V \\ DISCUSSION}

There is an unavoidable association between orthodontic treatment and facial esthetics that has motivated the investigation of diverse treatment options, and their effects, in hopes of discerning the optimal treatment for patients, both esthetically and functionally. The most advantageous treatment mechanics for the correction of Class II, division 1 malocclusions have long been central to these assessments. In efforts to determine the optimal treatment for patients with Class II, division 1 malocclusions, the present study evaluated two treatments used for the correction of this malocclusion (standard Edgewise mechanics with extractions and straightwire Edgewise mechanics with a MARA).

This study was a matched-pair design, which found the two treatment types to be esthetically indistinguishable at the end of treatment. This chapter discusses the findings and the influence (1) cephalometrics and (2) observer sex and background had on them. Though not directly tested, the cephalometric differences present at the end of treatment apparently exhibited no influence on the esthetic ratings. Sex and professional background, however, were found to influence the esthetic ratings given by our observers. These, along with other orthodontic limitations in improving facial esthetics (i.e., integumental variation), are further recognized below in this discussion. Lastly, a comparison of the present esthetic findings from orthodontic therapy with a functional orthopedic appliance (i.e., the MARA) and previous esthetic evaluations with functional appliances are discussed.

\section{Cephalometric Influences}

In this study, Edgewise treatment with and without a MARA produced essentially the same esthetic profile when evaluated by experienced orthodontists, graduate orthodontic students, and lay people. Significant cephalometric improvements were recognized following orthodontic treatment regardless of the two treatment alternatives. The Edgewise and MARA subjects were treated to a mean ANB of $2^{\circ}$ and $3^{\circ}$, respectively, which according to Steiner (1953) and Riolo et al. (1974) are within normal limits. Therefore, from a cephalometric standpoint, both treatment groups were treated successfully with virtually identical improvements. Both treatment groups had improved cephalometrically and esthetically at the end of treatment, but in different ways.

A statistically significant decrease in ANB $(\mathrm{P}<0.01)$ was seen in both groups after treatment. These changes consisted of greater maxillary restraint in 
the conventional Edgewise sample, with a statistically greater decrease in SNA (P $=0.009)$ than the MARA sample $\left(-3^{\circ}\right.$ vs. $-1^{\circ}$, respectively $)$, and more mandibular enhancement with the MARA sample, though not statistically significant. Though not directly tested, these cephalometric differences exhibited no influence on the esthetic ratings, with no systematic differences $(P=0.60)$ between the profile esthetics of the groups. This raises the question, how large of a difference in ANB is needed to have a perceptual esthetic effect?

The cephalometric differences apparently had no influence on the esthetic perceptions of the three observation panels, since the posttreatment esthetics were found to be indistinguishable. This was shown by (1) comparable mean improvements in average VAS scores (an increase of 10) for both treatment types from pre- to posttreatment and (2) the absence of a discernible difference between the two treatment types' posttreatment VAS scores (Figure A14). Therefore differences in the bony profiles brought about by the two treatments translated into equivalent improvements in the integumental profiles.

The inference is that the improved bony profile after treatment does not translate into equivalent improved integumental profiles. It seems that a few degrees of cephalometric difference present after treatment had no effect on the amount of esthetic improvements perceived; hence, a much greater difference in SNA, SNB, and ANB would be needed to allow a perceived difference in the esthetic comparison of the silhouettes. Either (1) greater treatment effects or (2) less heterogeneity in the samples would have brought about greater bony differences. The question remains, what amount of cephalometric difference would bring about an esthetic difference?

\section{$\underline{\text { Sex and Panel Background Influences }}$}

Although previous studies (e.g., Shaw 1981; De Smit and Dermaut 1984; Barrer and Ghafari 1985; Howells and Shaw 1985; O'Neill et al. 2000) have indicated that sex was not influential in observer ratings and others (e.g., Tedesco et al. 1983; Cochrane et al. 1999) have suggested that female observers are less critical and give higher attractiveness ratings than males, neither result was found to be true in the present investigation. In this study female observers generally scored patients' profiles comparatively lower than their male counterparts. This is in opposition to the findings of Barrer and Ghafari (1985), who found that observers' sex reflected no statistically significant influence on profile evaluations.

Another example of how sex was influential in this study is depicted in Figure 43, where female subjects were more-often judged to have positive 
changes in profile esthetics following orthodontic treatment than male subjects. In our evaluation, both female and male observers scored female profiles as having greater esthetic improvements than male profiles $(P=0.006)$. In contrast, Cross and Cross (1971) found the perceived attractiveness of adolescent female faces to be greater than the attractiveness of male faces by female raters only. These varied results suggest there is no clear trend in the literature concerning the influence sex has on panel esthetic ratings.

In addition to sex, the effect professional background can have on esthetic evaluations has been unclear in previous studies. Our results suggest that professional background did influence panel esthetic ratings; and, surprising to the investigators, the panel of experienced orthodontists was the least critical (i.e., higher VAS scores) of the three panels examined. Overall, the panel of lay people was more critical than the experienced orthodontists, but less critical than the panel of graduate orthodontic students, which was the most critical (i.e., lower VAS scores) of the three panels evaluated. Our findings support claims made by Tedesco et al. (1983), Phillips et al. (1992), and Spyropoulos and Halazonetis (2001) who reported panels of lay people being more critical in their esthetic evaluations than dental professionals; the present results, however, refute the findings of Prahl-Andersen et al. (1979), Kerr and O'Donell (1990), Bowman and Johnston (2000), and Kiekens et al. $(2005,2007)$ who claim the opposite. These, along with the current findings prove only that professional background plays a role in panel esthetic evaluations, although there is no general consensus 'to what end.'

\section{Limitations in Esthetic Improvement}

Esthetic improvements from orthodontic treatment are often hampered by integumental limitations. These integumental limitations can include changes in the nose, lips, chin and neck regions (all of which are outside the orthodontist's purview, yet are often included in the evaluation of treatment outcomes). Dentoskeletal correction can be accomplished with orthodontic therapy, while esthetic improvements can be hampered by growth or other modifications (e.g., weight gain) in areas unrelated to orthodontic treatment. Soft tissue changes incident to growth encompass a greater aspect of the soft tissue profile, including the nose and chin, as well as the lips (Subtelny 1961; Hambleton 1964; Vig and Cohen 1979), than changes due to dentoskeletal correction from orthodontic treatment. This could possibly explain why in this study a third (35\%) of all the VAS changes were found to be negative, when comparing the pre- and posttreatment silhouettes. Profile esthetic improvements could very well be limited to alterations in the integument (e.g., growth and weight gain), independent of orthodontic treatment. 


\section{Comparison of Previous Esthetic Evaluations with Functional Appliances}

As discussed previously in the review of the literature, the change in profile attractiveness of Class II, division 1 malocclusion patients has been previously measured following functional therapy with a Fränkel regulator (e.g., O'Neill et al. 2000), a Harvold activator (e.g., O'Neill et al. 2000), a Herbst appliance (e.g., Stock et al. 2006), and headgear (e.g., Stock et al. 2006). O'Neill et al. (2000) evaluated the change in profile attractiveness after 18 months of treatment with a Fränkel regulator, a Harvold activator, or an untreated control group. The results of $\mathrm{O}^{\prime} \mathrm{Neill}$ 's study showed there was no significant difference between the change in profile attractiveness of the untreated subjects and the subjects treated with the two functional appliances ( $\mathrm{O}^{\prime} \mathrm{Neill}$ et al. 2000). Stock et al. (2006) evaluated pre- and posttreatment silhouettes of growing Class II, division 1 patients treated with either a Herbst appliance or headgear prior to full orthodontic appliances. Both treatment groups exhibited significantly higher mean attractiveness scores after treatment $(P<0.05)$, but no significant difference was found at posttreatment between the two treatment groups $(\mathrm{P}=0.936)$. It was concluded from these findings that a growing subject with a Class II, division 1 malocclustion could achieve an equivalent profile improvement whether treated with either a Herbst appliance or with headgear prior to fixed appliances (Stock et al. 2006).

Although an untreated control group was not used in our study, the findings of O'Neill et al. (2000) and Stock et al. (2006) closely compare to the results found in the present evaluation comparing Edgewise treatment with and without a MARA. The average VAS scores for both treatment groups underwent a highly significant increase of 10 points from pre- to posttreatment, measured by paired $t$-tests with the MARA $(\mathrm{t}=16.6 ; \mathrm{df}=1,049 ; \mathrm{P}<0.0001)$ and without the MARA $(t=15.3 ; \mathrm{df}=1,049 ; \mathrm{P}<0.0001)$. The improvements, therefore, were highly significant and essentially identical for both treatment groups. Treatment type also was found to be far from significant $(P=0.60)$ when observers compared the pre- and posttreatment silhouettes. Overall, in agreement with O'Neill et al. (2000) and Stock et al. (2006), functional therapy in Class II, division 1 malocclusion patients has yet to be shown to create more attractive profiles.

Interesting assessments involving esthetic ratings and cephalometric findings were found for both the current study and Stock et al. (2006). Stock et al. (2006) found an intriguing relationship between esthetic scores and skeletal discrepancy. To their surprise, lower profile scores were not assigned to subjects having larger skeletal discrepancies (Stock et al. 2006). In this study, a marginally-significant difference in ANB was found at the end of treatment for 
the two treatment groups $(P=0.03)$, where the mean ANB was $3^{\circ}$ for the MARA sample and just over $2^{\circ}$ for the Edgewise sample. This was due to the MARA sample being treated to a slightly higher SNA $\left(79^{\circ} v s .78^{\circ}\right)$. Surprising to the current investigators, the cephalometric difference between the treatment groups had no effect on the esthetic ratings. Both of these assessments made by the current study and Stock et al. (2006) once again question, how large of a cephalometric difference is needed to affect the esthetic perceptions of the profile? 


\section{CHAPTER VI SUMMARY AND CONCLUSIONS}

The effects that orthodontic therapy can have on facial esthetics have long been a concern of the orthodontic community. Considerations involving orthodontic treatment and facial esthetics have directed the investigation of the optimal treatment, especially in the correction of Class II, division 1 malocclusions, both esthetically and functionally. The present retrospective study compared two groups of patients, one consisting of a sample of 30 consecutively treated American white adolescents treated with functional orthopedic therapy with a MARA-in combination with preangulated Edgewise appliances. The second, matched group was treated using standard Edgewise mechanics alone. This study investigated the perceived posttreatment facial esthetics of American white adolescents with Class II, division 1 malocclusions treated by one of these two methods. Lay adults, graduate orthodontic students, and experienced orthodontists rated the level of profile attractiveness before and after orthodontic treatment. The question was whether the use of a MARA in combination with Edgewise mechanics yielded a more esthetic facial profile than Edgewise treatment alone. Major findings are:

1. Both treatment groups had a significantly improved level of profile attractiveness at the end of treatment; indeed, the improvements in VAS scores were virtually identical.

2. Comparing the perceptions of female and male observers, female observers gave lower VAS score on average than the male observers in this study. We attribute the sex difference to the particular raters in this study rather than any fundamental sex difference in perception.

3. A much higher percentage of cases treated with Edgewise appliances alone required premolar extractions, probably because they were older, with less growth potential.

4. There was no difference between the two groups in the perceived amounts of profile change following treatment.

5. Comparing the perceptions of the three observation panels, the experienced orthodontists gave the highest (most favorable) VAS scores on average, followed by the lay people, then followed by the graduate orthodontic students who gave lowest VAS scores on average.

6. While the MARA promotes mandibular growth and reduces the need to restrain maxillary growth, these skeletal benefits seem to be obscured by the variability in the integumental conditions so that this protocol does not produce a readily-discernible benefit to the patient's facial profile across the sample. 


\section{LIST OF REFERENCES}

Aitken RCB. Measurement of feelings using visual analogue scales. Proc R Soc Med 1969;62:989-93.

Allen-Noble PS, Allesee Orthodontic Appliances/Pro. Clinical management of the MARA. 2002.

Angle EH. Treatment of malocclusion of the teeth and fractures of the maxillae, Angle's system, 6th ed. Philadelphia: SS White Dental Mfg Company; 1900.

Angle EH. Malocclusion of the teeth, 7th ed. Philadelphia: SS White Dental Mfg Company; 1907.

Athanasiou AE. Orthodontic cephalometry. London: Mosby-Wolfe; 1995.

Barrer JG, Ghafari J. Silhouette profiles in the assessment of facial esthetics: a comparison of cases treated with various orthodontic appliances. Am J Orthod 1985;87:385-91.

Baum AT. Orthodontic treatment and the maturing face. Angle Orthod 1966;36:121-135.

Baumgarten AG. Aesthetica. Paris: L'herne; 1989.

Bernstein IH, Lin TD, McClellan P. Cross- vs. within-racial judgments of attractiveness. Percept Psychophys 1982;32:495-503.

Berscheid E, Walster E. Physical attractiveness. In: Berkowitz L, editor. Advances in experimental social psychology, vol 7. New York: Academic Press; 1974. p.157-215.

Birkeland K, Boe OE, Wisth PJ. Relationship between occlusion and satisfaction with dental appearance in orthodontically treated and untreated groups. A longitudinal study. Eur J Orthod 2000;22:509-18.

Bowman SJ, Johnston LE. The esthetic impact of extraction and nonextraction treatments on Caucasian patients. Angle Orthod 2000;70:3-10.

Burstone CJ. The integumental profile. Am J Orthod 1958;44:1-25. 
Case CS. Facial and oral deformities. Chicago: CS Case and Company; 1896.

Case CS. A practical treatise on the technics and principles of dental orthopedia and correction of cleft palate, 2nd ed. Chicago: CS Case and Company; 1922.

Cochrane S, Cunningham S, Hunt N. A comparison of the perception of facial profile by the general public and three groups of clinicians. Int J Adult Orthod Orthognath Surg 1999;14:291-5.

Courtney MD, Leigh TJ. Evidence-based orthodontics. Am J Orthod Dentiofacial Orthop 2001;120:18A-19A.

Cross JF, Cross J. Age, sex and race, and the perception of facial beauty. Dev Psychol 1971;5:433-9.

De Laat RC. Orthodontics and the facial profile. Amsterdam: Graduated Press; 1974.

De Smit A, Dermaut L. Soft-tissue profile preference. Am J Orthod 1984;86:6773.

Dion KK, Berscheid E. Physical attractiveness and peer perception among children. Sociometry 1974;37:1-12.

Dongieux J, Sassouni V. The contribution of mandibular positioned variation to facial esthetics. Angle Orthod 1980;50:334-9.

Downs WB. Variations in facial relations: their significance in treatment and prognosis. Am J Orthod 1948;34:812-40.

Edler RJ. Background considerations to facial aesthetics. J Orthod 2001;28:15968.

Evan R, Shaw W. Preliminary evaluation of an illustrated scale for rating dental attractiveness. Eur J Orthod 1987;9:314-8.

Faure JC, Rieffe C, Maltha JC. The influence of different facial components on facial aesthetics. Eur J Orthod 2002;24:1-7.

Flores-Mir C, Silva E, Barriga MI, Lagravère MO, Major PW. Layperson's perception of smile aesthetics in dental and facial views. J Orthod 2004;31:204-9. 
Gebeck TR. Analysis - concepts and values: Part 1. J Charles H Tweed Int Foundation 1989;17:19-48.

Giddon DB. Commentary on ratings of facial attractiveness after functional appliance treatment. Am J Orthod Dentofacial Orthop 2000;118:371-6.

Graber TM, Vanarsdall RL Jr, Vig KW. Orthodontics: current principles and techniques, 4th ed. St. Louis: Elsevier Inc; 2005.

Hambleton RS. The orthodontic curtain. Angle Orthod 1963;33:294-8.

Hambleton RS. Soft tissue covering of the skeletal face as related to orthodontic problems. Am J Orthod 1964;50:405-20.

Hershey HG. Incisor tooth retraction and subsequent profile change in postadolescent female patients. Am J Orthod 1972;61:45-54.

Herzberg BL. Facial esthetics in relation to orthodontic treatment. Angle Orthod 1952;22:3-22.

Hess DR. Retrospective studies and case reviews. Respir Care 2004;49:1171-4.

Holdaway RA. Changes in relationship of points A and B during orthodontic treatment. Am J Orthod 1956;42:176-93.

Holdaway RA. A soft-tissue cephalometric analysis and its use in orthodontic treatment planning. Part I. Am J Orthod 1983;84:1-28.

Holdaway RA. A soft-tissue cephalometric analysis and its use in orthodontic treatment planning. Part II. Am J Orthod 1984;85:279-93.

Howells DJ, Shaw WC. The validity and reliability of ratings of dental and facial attractiveness for epidemiologic use. Am J Orthod 1985;88:402-8.

http://en.wikipedia.org/wiki/Beauty. Accessed August 16, 2006.

http://en.wikipedia.org/wiki/Image:Athena_Parthenos_Altemps_Inv8622.jpg. Accessed August 28, 2007.

http://en.wikipedia.org/wiki/Image:Da_Vinci_Vitruve_Luc_Viatour.jpg. Accessed January 24, 2008. 
http://en.wikipedia.org/wiki/Image:Doryphoros.jpg. Accessed August 29, 2007.

http://en.wikipedia.org/wiki/Image:Venus_de_Milo_Louvre_Ma399_n4.jpg. Accessed August 29, 2007.

http://en.wikipedi.org/w/index.php?title=Image:Belvedere_Apollo_PioClementino_Inv1015.jpg\&printable=yes. Accessed August 29, 2007.

http://en.wikipedi.org/w/index.php?title=Image:Michelangelos_David.jpg\&pri ntable=yes. Accessed August 29, 2007.

http://en.wikipedi.org/w/index.php?title=Image:St_George_Donatello_Orsan michele_n1.jpg\&printable=yes. Accessed August 29, 2007.

Hume D. 'Of Tragedy' in essays, literature, moral and political. London: Oxford University Press; 1963.

Hungerford MW. Molly Bawn. London: Smith, Elder \& Company; 1878.

Huskinsson EC. Visual analogue scales. In: Melzack R, editor. Pain measurement and assessment. New York: Raven Press; 1983. p.33-40.

Hutcheson F. An inquiry into the original of our ideas of beauty and virtue. Indianapolis: Liberty Fund; 2004.

Isaac S, Michael WB. Handbook in research and evaluation. San Diego: Edits Publishers; 1971.

Johnston LE. A simplified approach to prediction. Am J Orthod 1975;67:253-7.

Kant I. The critique of judgement. London: Oxford University Press; 1973.

Kerr WJS, O'Donell JM. Panel perception of facial attractiveness. Br J Orthod 1990;17:299-304.

Kiekens RMA, Maltha JC, van t Hof MA, Kuijpers-Jagtman AM. A measuring system for facial aesthetics in Caucasian adolescents: reproducibility and validity. Eur J Orthod 2005;27:579-84.

Kiekens RMA, van 't Hof MA, Straatman H, Kuijpers-Jagtman AM, Maltha JC. Influence of panel composition on aesthetic evaluation of adolescent faces. Eur J Orthod 2007;29:95-99. 
King EW. Variations in profile change and their significance in timing treatment. Angle Orthod 1960;30:141-53.

Kolar JC, Salter EM. Craniofacial anthropometry: practical measurement of the head and face for clinical, surgical, and research use. Springfield: Charles C. Thomas Publisher; 1997.

Langlois JH. From the eye of the beholder to behavioral reality: the development of social behaviors and social relations as a function of physical attractiveness. In: Herman CP, Zanna MP, Higgins ET, editors. Physical appearance, stigma, and social behavior: the Ontario symposium. Hillsdale: Erlbaum; 1986. p.23-51.

Langlois JH, Roggman LA, Casey RJ, Ritter JM, Rieser-Danner LA, Jenkins VY. Infant preferences for attractive faces: rudiments of a stereotype? Dev Psychol 1987;23:363-9.

Lapatin KDS. Book Reviews. Art Bulletin 1997;79:148-56.

Lindquist JT. The lower incisor-its influence on treatment and esthetics. 1958;44:112-40.

Lines PA, Lines RR, Lines CA. Profilemetrics and facial esthetics. Am J Orthod 1978;73:648-57.

Lundström A, Woodside DG, Popovich F. Panel assessments of facial profile related to mandibular growth and direction. Eur J Orthod 1987;9:271-8.

Lundström A, Popovich F, Woodside DG. Panel assessments of the facial frontal views as related to mandibular growth direction. Eur J Orthod 1989;11:290-7.

Maret SM. Attractiveness ratings of photographs of blacks by Cruzans and Americans. J Psychol 1983;115:113-6.

Maret SM, Harling CA. Cross cultural perceptions of physical attractiveness: ratings of photographs of Whites by Cruzans and Americans. Perceptual Motor Skills 1985;60:163-6.

McNamara JA Jr, Brudon WL. Orthodontics and orthopedic treatment in the mixed dentition. Ann Arbor: Needham Press, Inc; 1993. 
Mergen JL, Southard KA, Dawson DV, Fogle LL, Casko JS, Southard TE.

Treatment outcomes of growing Class II division 1 patients with varying degrees of anteroposterior and vertical dysplasias, Part 2. Profile silhouette evaluation. Am J Orthod Dentofacial Orthop 2004;125:457-62.

Merrifield LL. The profile line as an aid in critically evaluating facial esthetics. Am J Orthod 1966;52:804-22.

Merrifield LL. Analysis-concepts and values: Part II. J Charles H Tweed Int Foundation 1989;17:49-64.

Merrifield LL. Differential diagnosis. Semin Orthod 1996;2:241-53.

Moore AW. Observations on facial growth and its clinical significance. Am J Orthod 1959;45:399-423.

Moyers RE, Riolo ML, Guire KE, Walnright RL, Bookstein FL. Differential diagnosis of Class II malocclusions. Part 1. Facial types associated with Class II malocclusions. Am J Orthod 1980;78:477-94.

O'Neill K, Harkness M, Knight R. Ratings of profile attractiveness after functional appliance treatment. Am J Orthod Dentofacial Orthop 2000;118:371-6.

Pangrazio-Kulbersh V, Berger JL, Chermak DS, Kaczynski R, Simon ES, Haerin A. Treatment effects of the mandibular anterior repositioning appliance on patients with Class II malocclusions. Am J Orthod Dentofacial Orthop 2003;123:286-95.

Peck H, Peck S. A concept of facial esthetics. Angle Orthod 1970;40:284-317.

Peerlings RHJ, Kuijpers-Jagman AM, Hoeksma JB. A photographic scale to measure facial aesthetics. Eur J Orthod 1995;17:101-9.

Phillips C, Trentini CJ, Douvartzidis N. The effect of treatment on facial attractiveness. J Oral Maxillofac Surg 1992;50:590-4.

Phillips C, Tulloch C, Dann C. Rating of facial attractiveness. Community Dent Oral Epidemiol 1992;20:214-20.

Powell N, Humphries B. Proportions of the aesthetic face. New York: CM Thieme-Stratton Inc; 1984. 
Prahl-Anderson B, Boersma H, van der Linden FPGM, Moore AW. Perceptions of dentofacial morphology by laypersons, general dentists, and orthodontists. J Am Dent Assoc 1979;98:209-12.

Proffit WR, Phillips C, Douvartzidis N. A comparison of outcomes of orthodontic and surgical-orthodontic treatment of Class II malocclusion in adults. Am J Orthod Dentofacial Orthop 1992;101:556-65.

Proffit WR, Fields HW Jr, Sarver DM. Contemporary Orthodontics, 4th ed. St. Louis: Mosby; 2007.

Revill SI, Robinson JO, Rosen M, Hogg MIJ. The reliability of a linear analogue for evaluating pain. Anesthesia 1976;31:1191-8.

Ricketts RM. Foundation for cephalometric communication. Am J Orthod 1960;46:330-57.

Ricketts RM. The biological significance of the divine proportion and Fibonacci series. Am J Orthod 1982;81:351-70.

Riolo ML, Moyers RE, McNamara JA Jr, Hunter WS. An atlas of craniofacial growth: cephalometric standards from the University School Growth Study, the University of Michigan. Monograph 2, Craniofacial Growth Series. Ann Arbor: Center for Human Growth and Development, University of Michigan; 1974.

Rondeau B. MARA appliance. Funct Orthod 2002;19:4-12,14-16.

Rosner BA. Fundamentals of biostatistics. Pacific Grove: Duxbury; 2000. p.40712.

Rudee DA. Proportional profile changes concurrent with orthodontic therapy. Am J Orthod 1964;50:421-34.

Salzmann JA. Limitations of roentgenographic cephalometrics. Am J Orthod 1964;50:169-88.

Samuels CA, Ewy R. Aesthetic perception of faces during infancy. Br J Dev Psychol 1985;3:221-8.

Scott J, Huskisson EC. Graphic representation of pain. Pain 1976;2:175-84. 
Shaw WC, Gabe MJ, Jones BM. The expectations of orthodontic patients in South Wales and St. Louis, Missouri. Br J Orthod 1979;6:203-5.

Shaw WC. The influence of children's dentofacial appearance on their social attractiveness as judged by peers and lay persons. Am J Orthod 1981;79:399-415.

Shaw WC, Rees G, Dawe M, Charles CR. The influence of dentofacial apperarnce on the social attractiveness of young adults. Am J Orthod 1985;87:21-6.

Shell TL, Woods MG. Perception of facial esthetics: a comparison of similar Class II cases treated with attempted growth modification or later orthognathic surgery. Angle Orthod 2003;73:365-73.

Shell TL, Woods MG. Facial aesthetics and the divine proportion: a comparison of surgical and non-surgical Class II treatment. Aust Orthod J 2004;20:5163.

Shelly AD, Southard TE, Southard KA, Casko JS, Jakobsen JR, Fridrich KL, et al. Evaluation of profile esthetic change with mandibular advancement surgery. Am J Orthod Dentofacial Orthop 2000;117:630-7.

Sokal RR, Rohlf FJ. Biometry: the principles and practice of statistics in biological research, 3rd ed. San Francisco: WH Freeman and Company; 1995.

Spyropoulos MN, Halazonetis DJ. Significance of the soft tissue profile on facial esthetics. Am J Orthod Dentofacial Orthop 2001;119:464-71.

Steiner CC. Cephalometrics for you and me. Am J Orthod 1953;39:729-55.

Stewart A. The canon of Polykleitos: a question of evidence. J Hellenic Studies 1978;98:122-31.

Stock S, Southard K, Sloss E, Qian F, Mann K. Profile attractiveness following treatment with the Herbst appliance versus headgear. J Dent Res 2006; special issue A:Abstract 790.

Stoner MM. A photometric analysis of the facial profile. Am J Orthod 1955;41:453-69.

Subtelny JD. The soft tissue profile, growth and treatment changes. Angle Orthod 1961;31:105-22. 
Sutter RE Jr, Turley PK. Soft tissue evaluation of contemporary Caucasian and African American female facial profiles. Angle Orthod 1998;68:487-96.

Tedesco LA, Albino JE, Cunat JJ, Green LJ, Lewis EA, Slakter MJ. A dental-facial attractiveness scale. Part 1 . Reliability and validity. Am J Orthod 1983;83:38-43.

Thakera JN, Iwawaki S. Cross-cultural comparisons in interpersonal attraction of females toward males. J Social Psychol 1979;108:121-2.

Tremouth MJ. Petrus Camper (1972-1789): originator of cephalometrics. Dent Hist 2003;40:3-14.

Tukey JW. Exploratory data analysis. Massachusetts: Addison-Wesley, Reading; 1977.

Tweed CH. The Frankfort-mandibular incisor angle in orthodontic diagnosis, treatment planning and prognosis. Angle Orthod 1954;24:121-69.

Tweed CH. Clinical orthodontics. St. Louis: Mosby; 1966.

Tweed $\mathrm{CH}$. The diagnostic facial triangle in the control of treatment objectives. Am J Orthod 1969;55:651-67.

Vaden JL, Harris EF, Sinclair P. A comparison of posterior to anterior facial height changes in treated Class I and Class II patients. Am J Orthod Dentofacial Orthop 1994;105:438-43.

Vig KW, Weyant R, O'Brien K, Bennett E. Developing outcome measures in orthodontics that reflect patient and provider values. Semin Orthod 1999;5:85-95.

Vig PS, Cohen AM. Vertical growth of the lips: A serial cephalometric study. Am J Orthod 1979;75:405-15.

Wahl N. Orthodontics in 3 millennia. Chapter 7: Facial analysis before the advent of the cephalometer. Am J Orthod Dentofacial Orthop 2006;129:293-8.

Wewers ME, Lowe NK. A critical review of visual analogue scales in the measurement of clinical phenomena. Res Nurs Health 1990;13:227-36. 
Winer BJ, Brown DR, Michels KM. Statistical principles in experimental design, 3rd ed. New York: McGraw-Hill Book Company; 1991.

Wisth PJ. Soft tissue response to upper incisor retraction in boys. Br J Orthod 1974;1:199-204.

Woolf CM. Principles of biometry. Princeton, NJ: D. Van Nostrand Company, Inc., 1968.

Worms FW, Isaacson RJ, Speidel TM. Surgical orthodontic treatment planning: profile analysis and mandibular surgery. Angle Orthod 1976;46:1-25.

Wuerpel E. Ideals and idealism. Angle Orthod 1931;1:14-31.

Wuerpel E. On facial balance and harmony. Angle Orthod 1937;7:81-9. 
APPENDIX

SUBJECTS' SILHOUETTES 


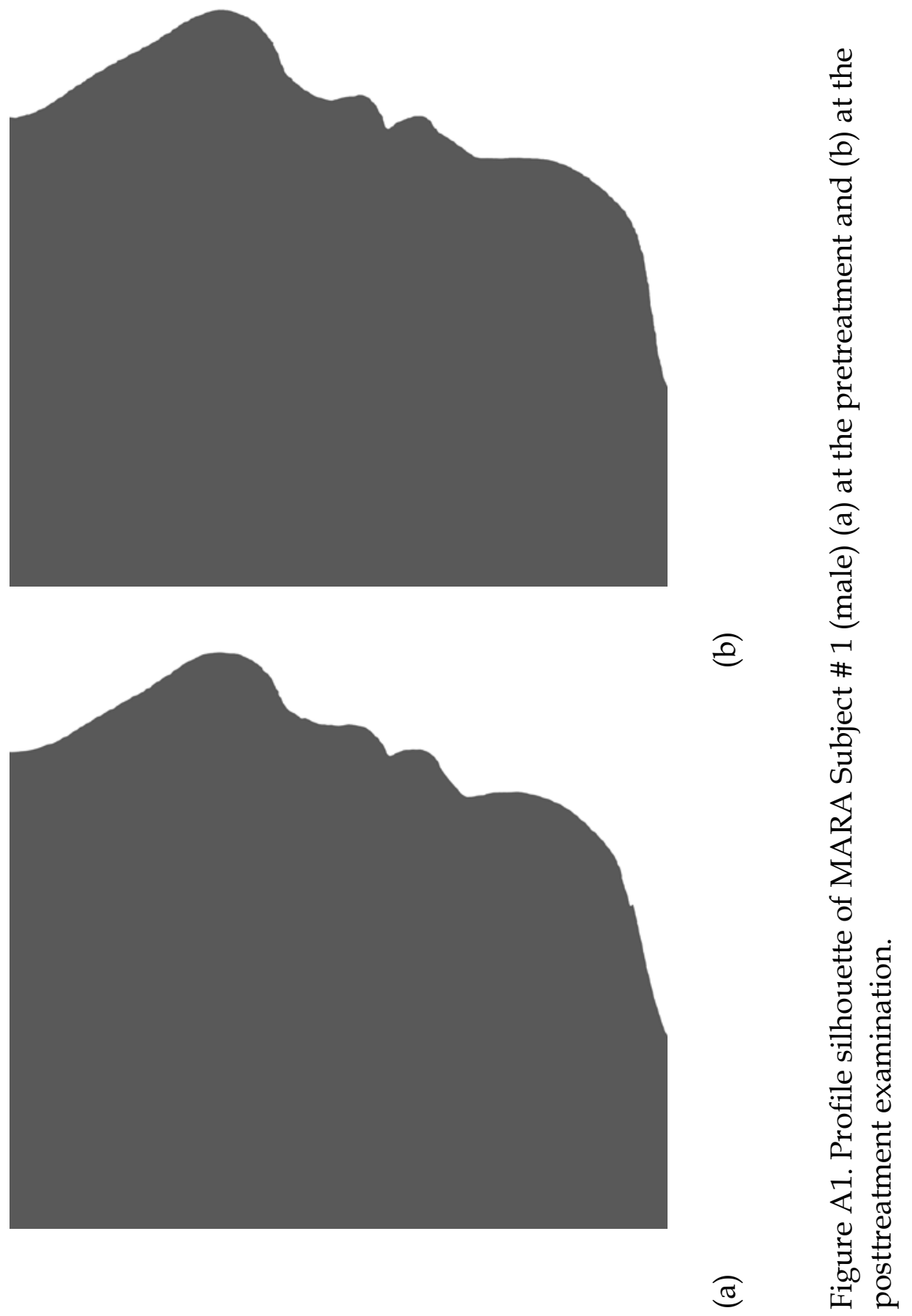




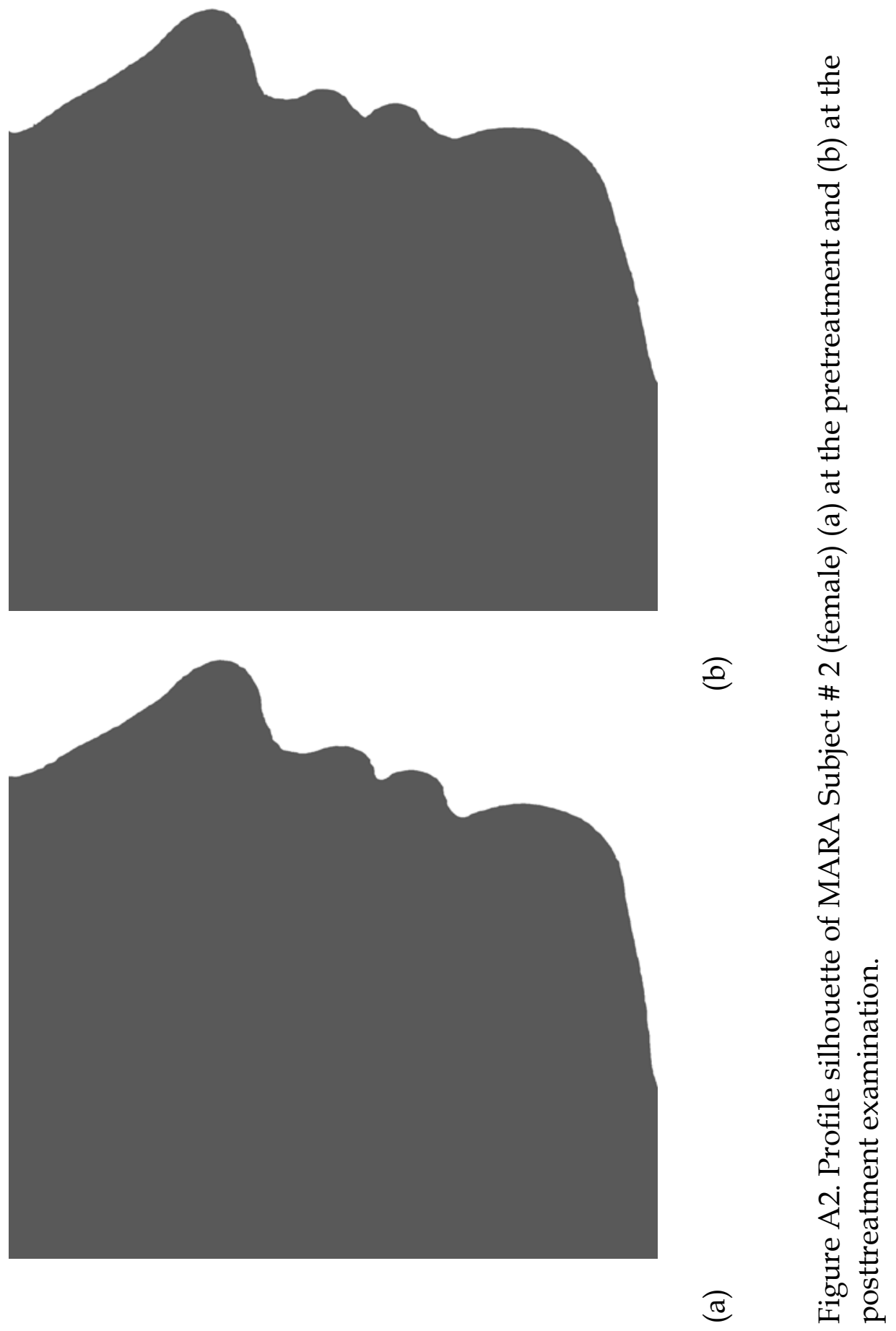




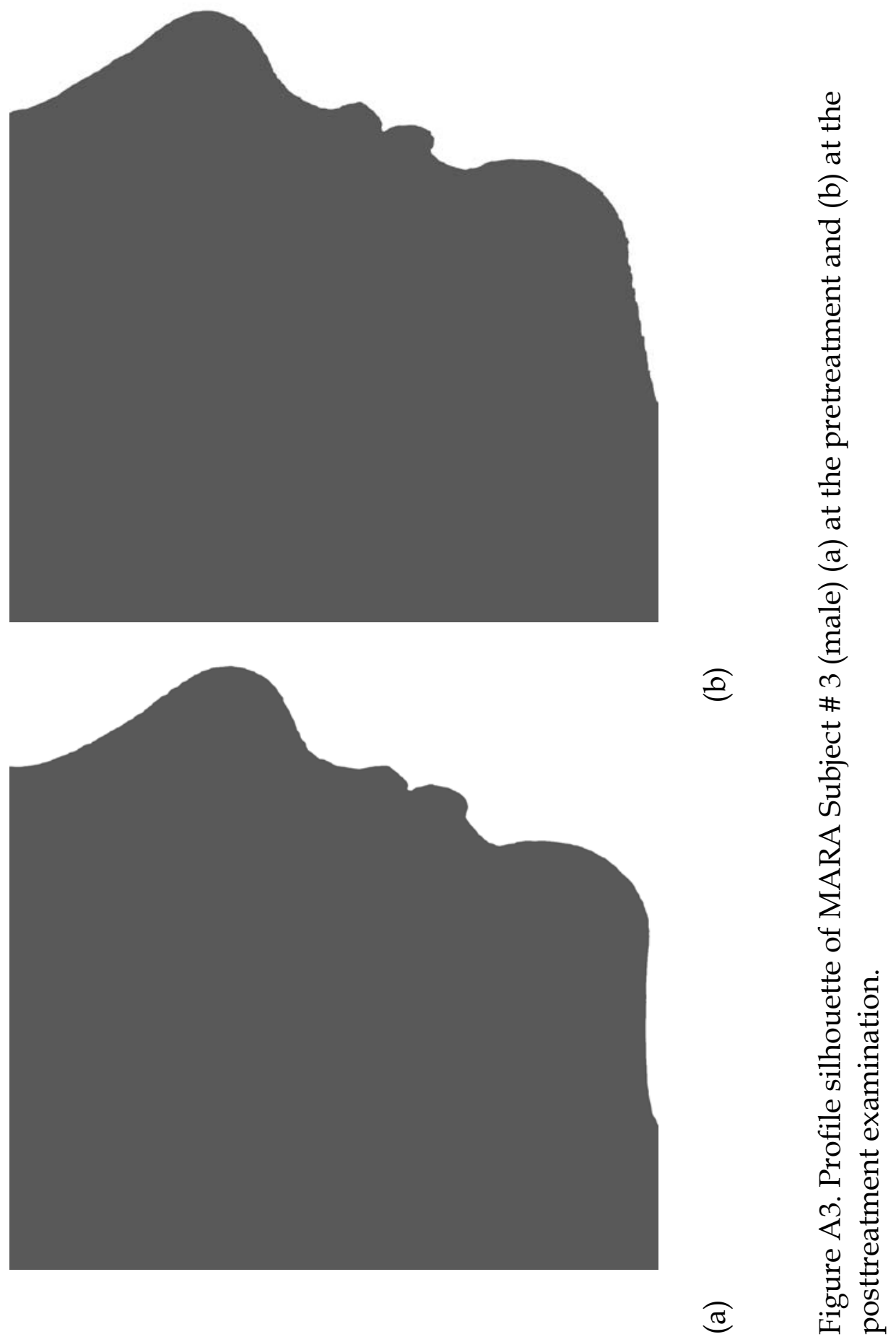




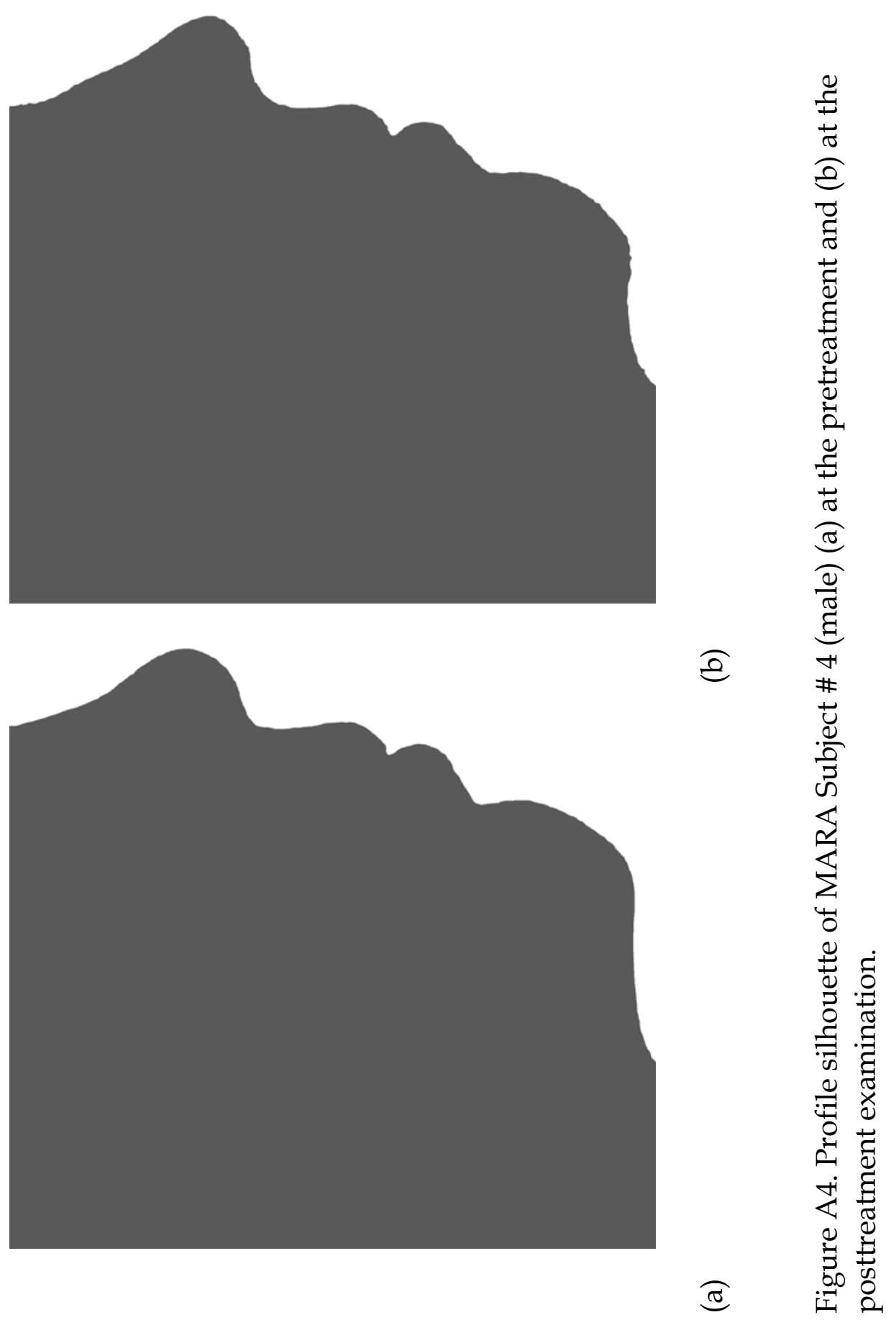




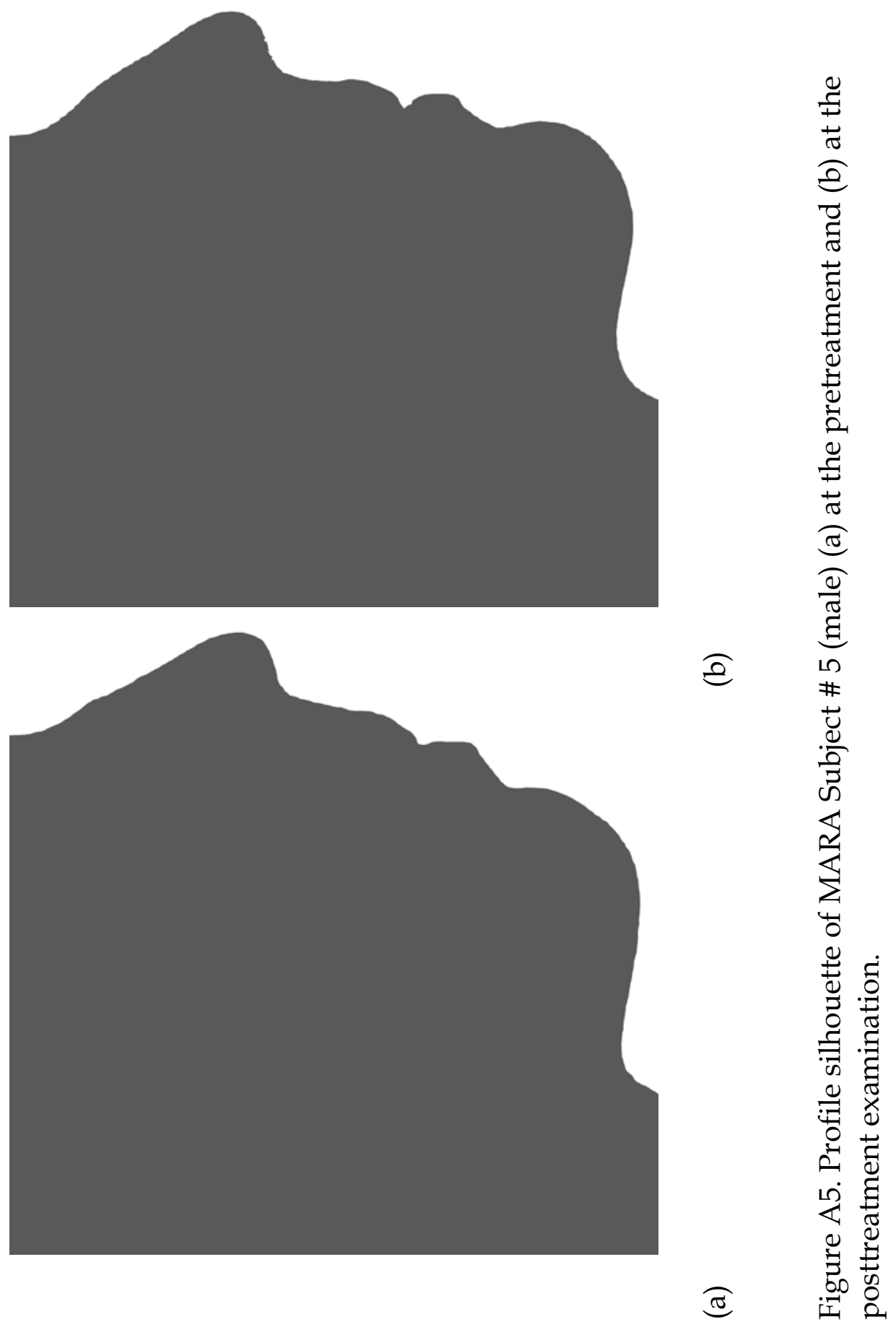




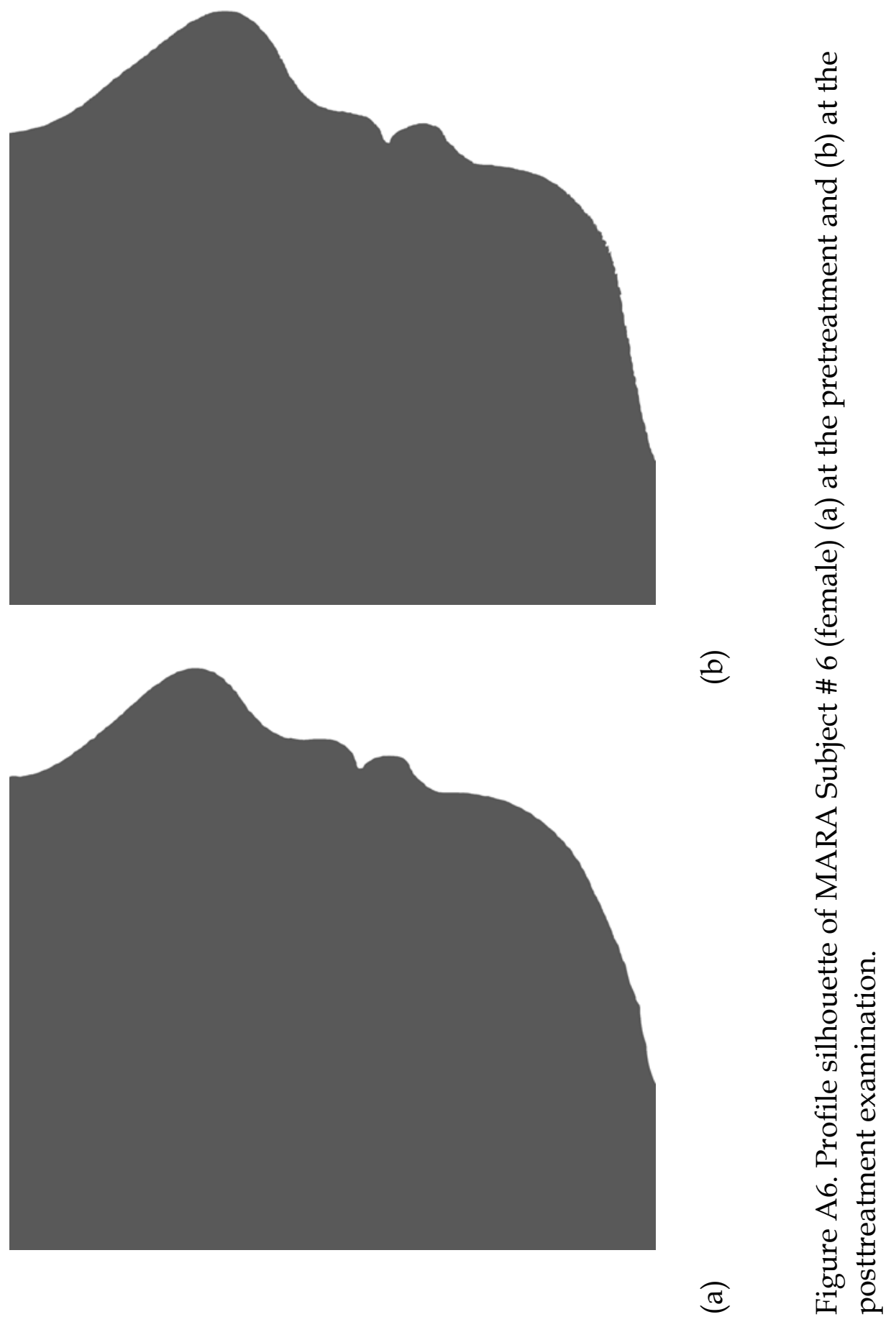




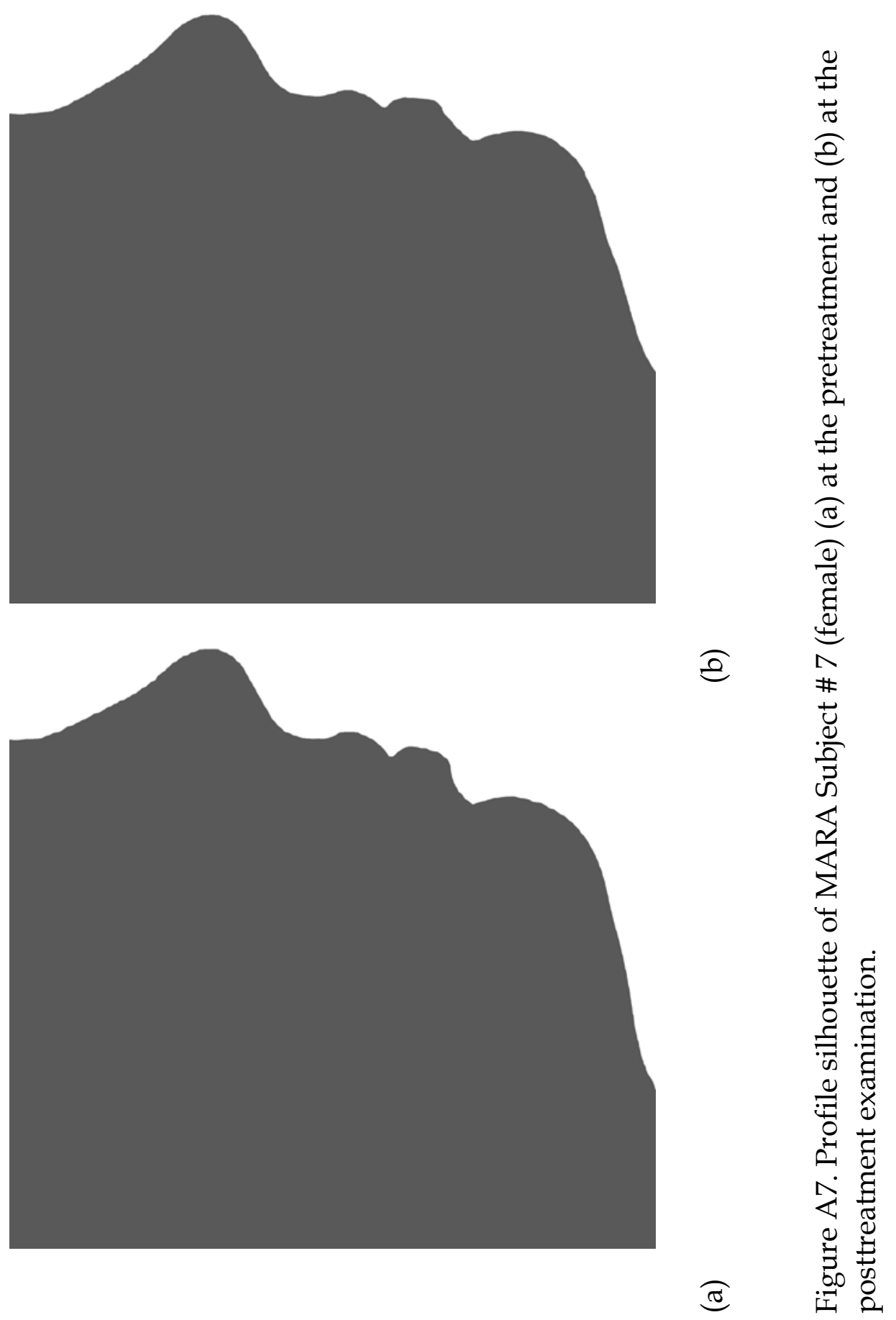




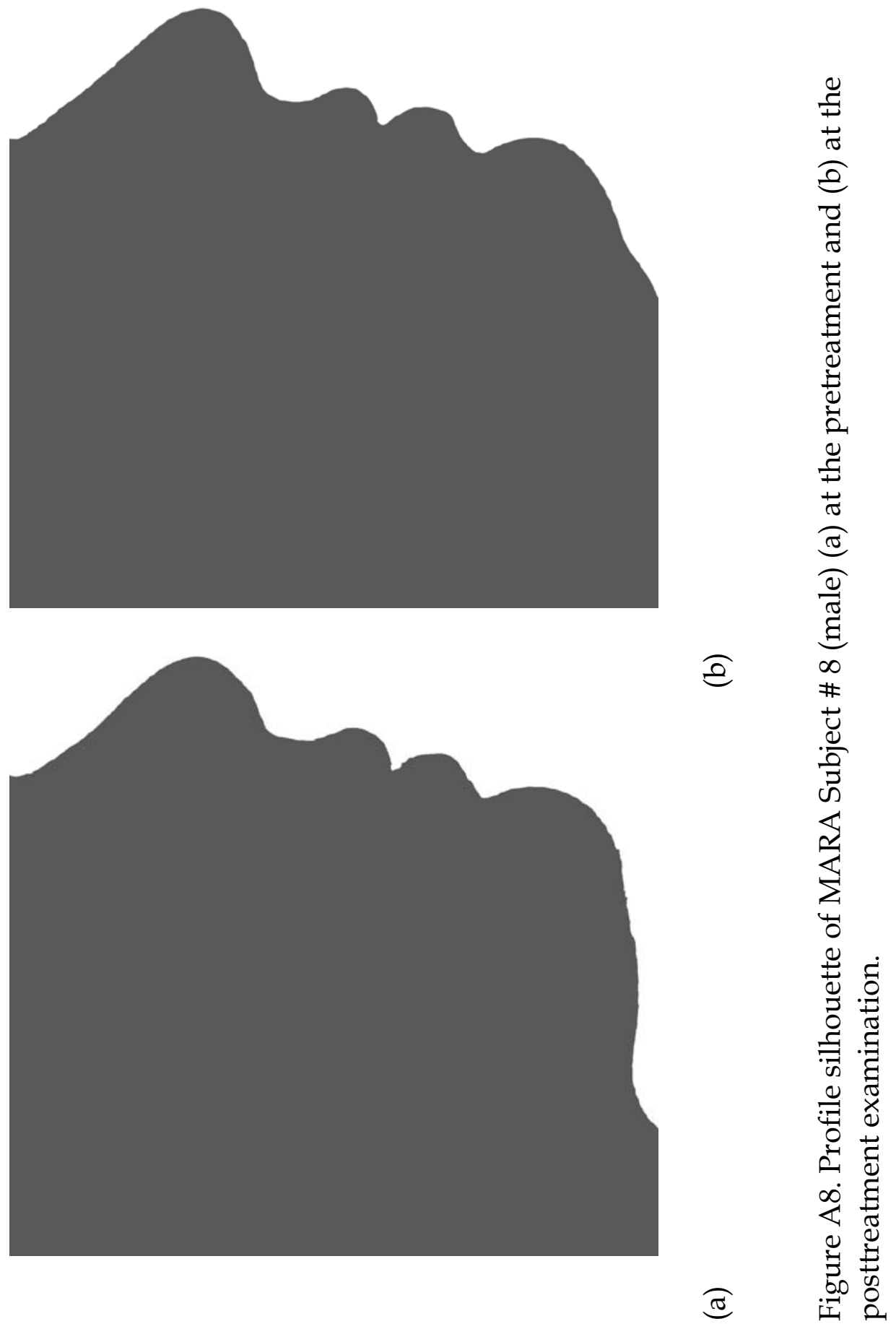




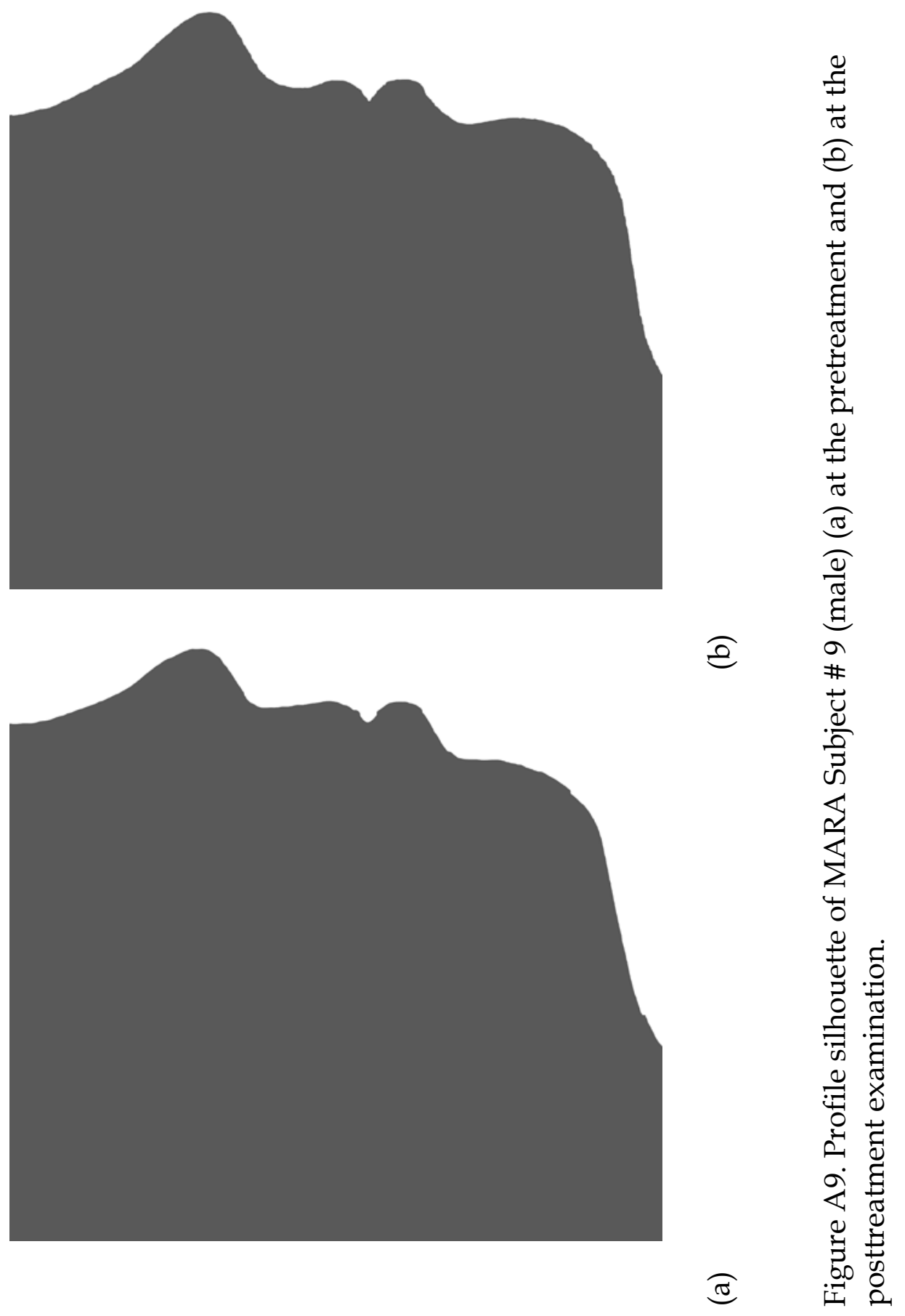




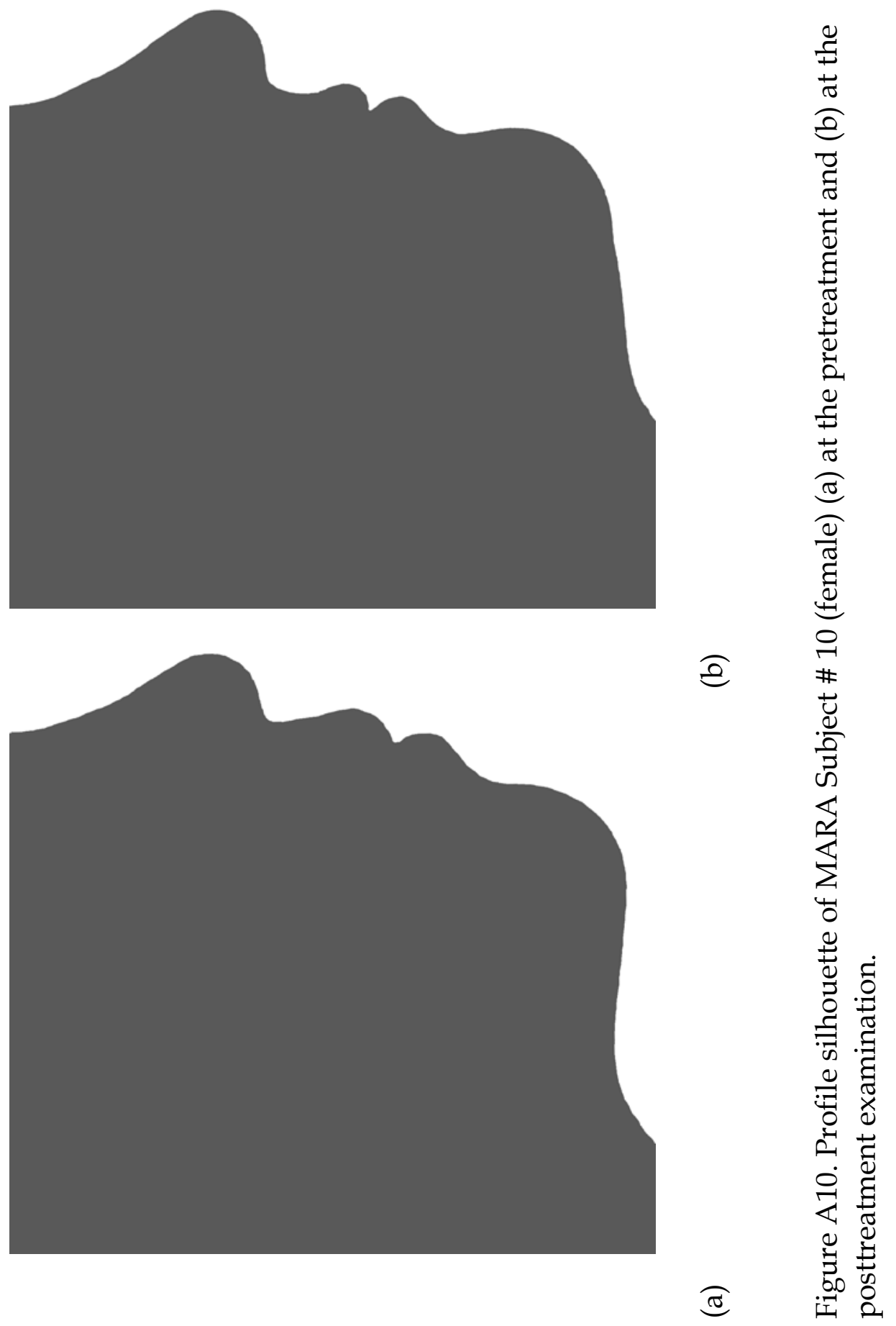




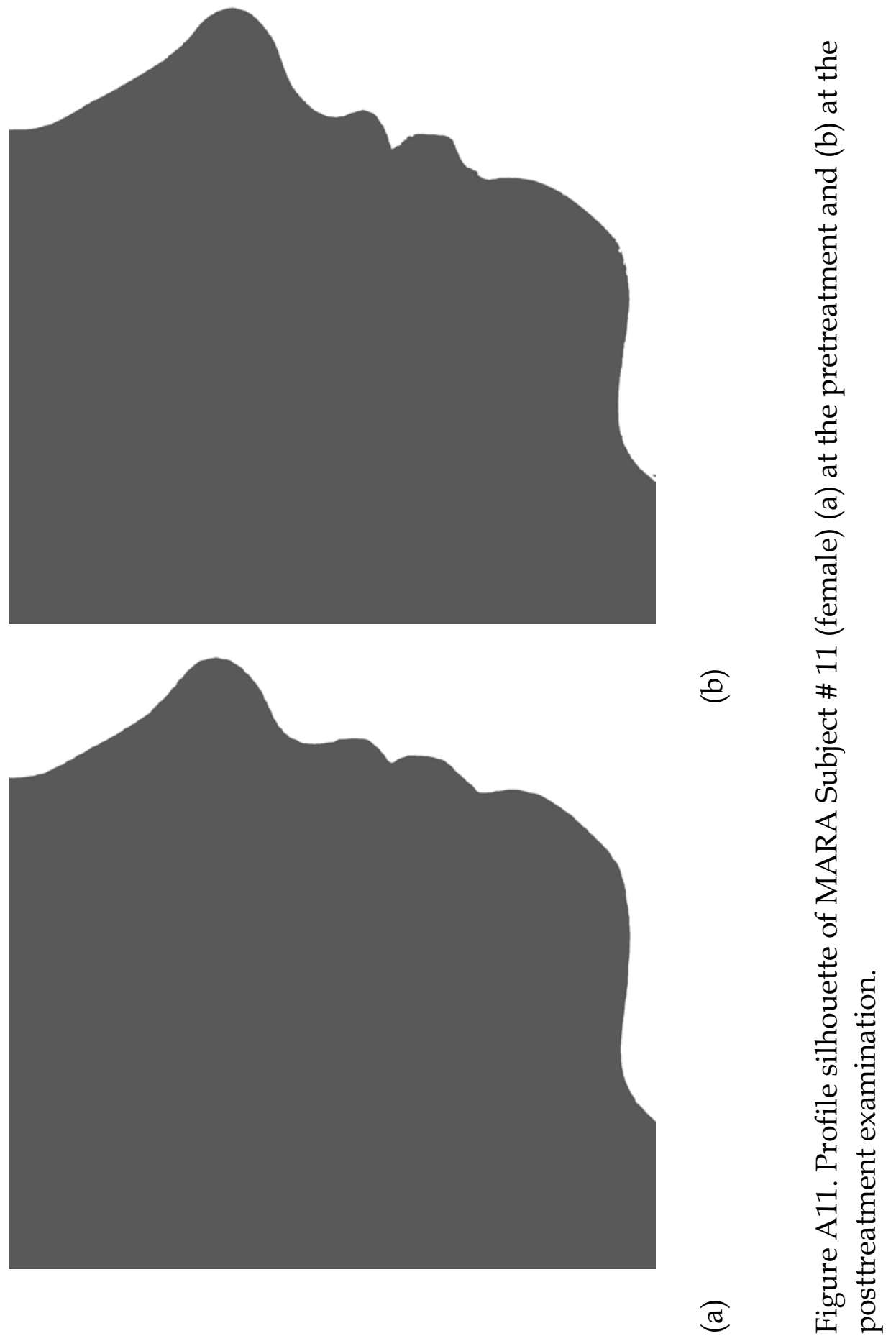




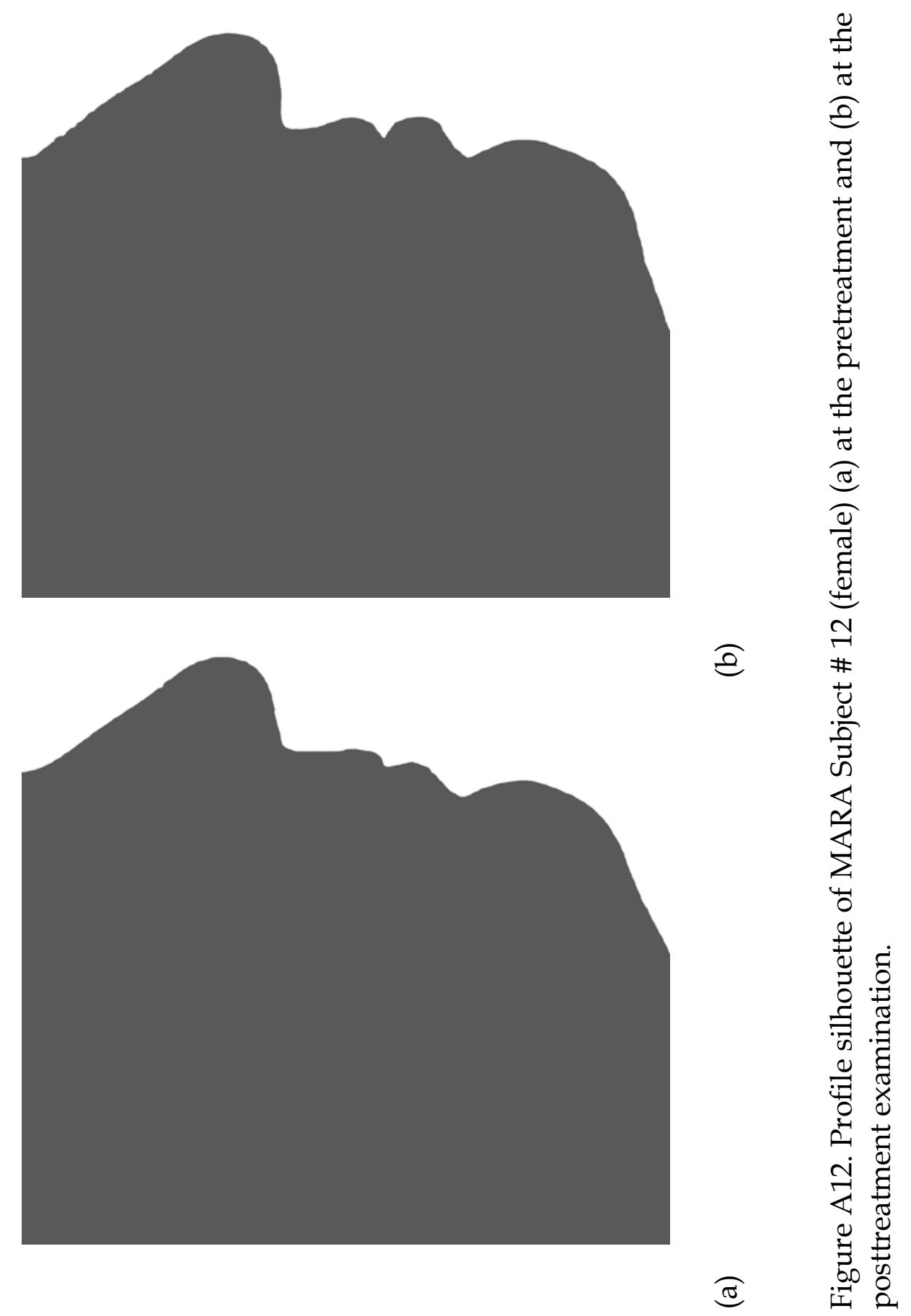




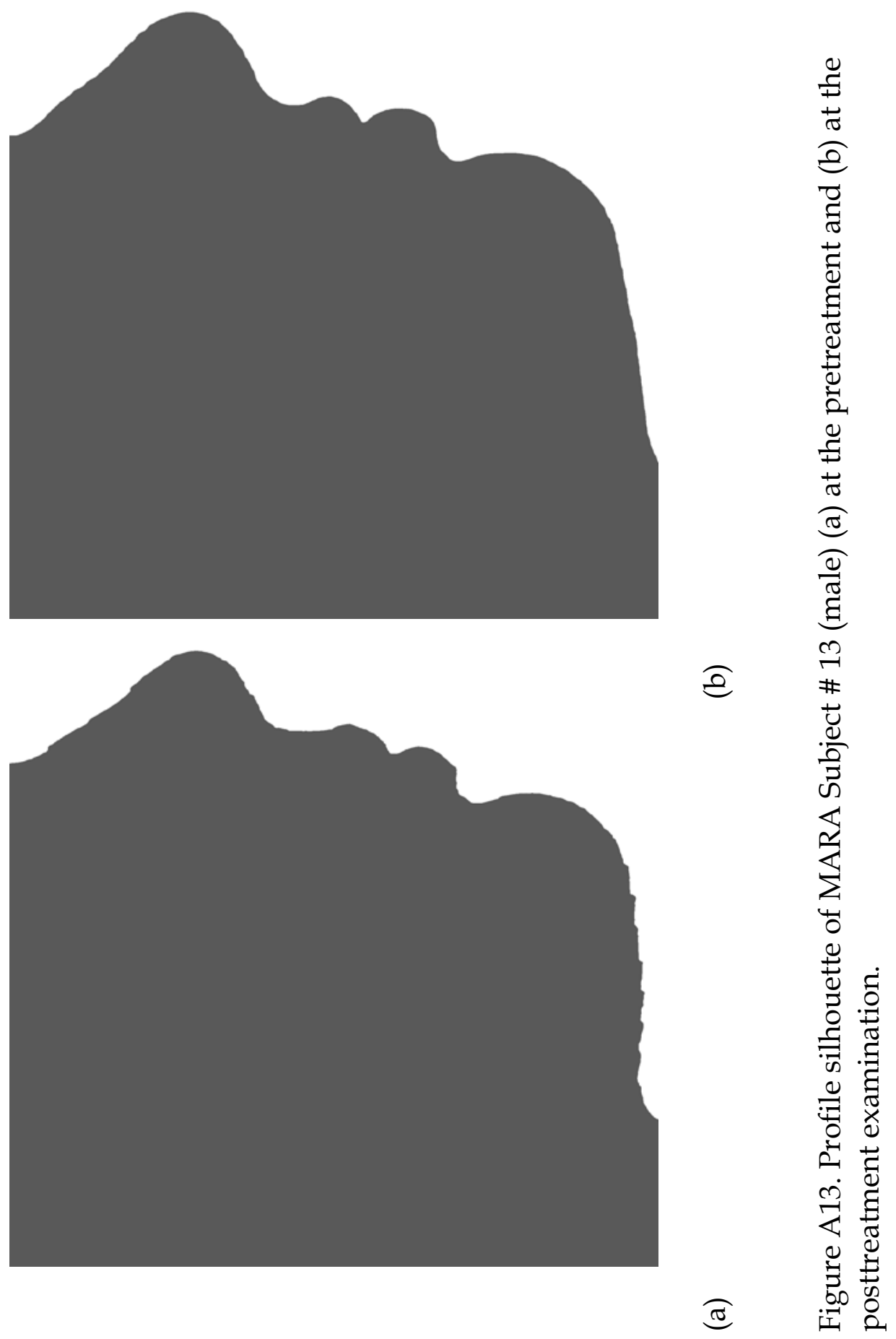




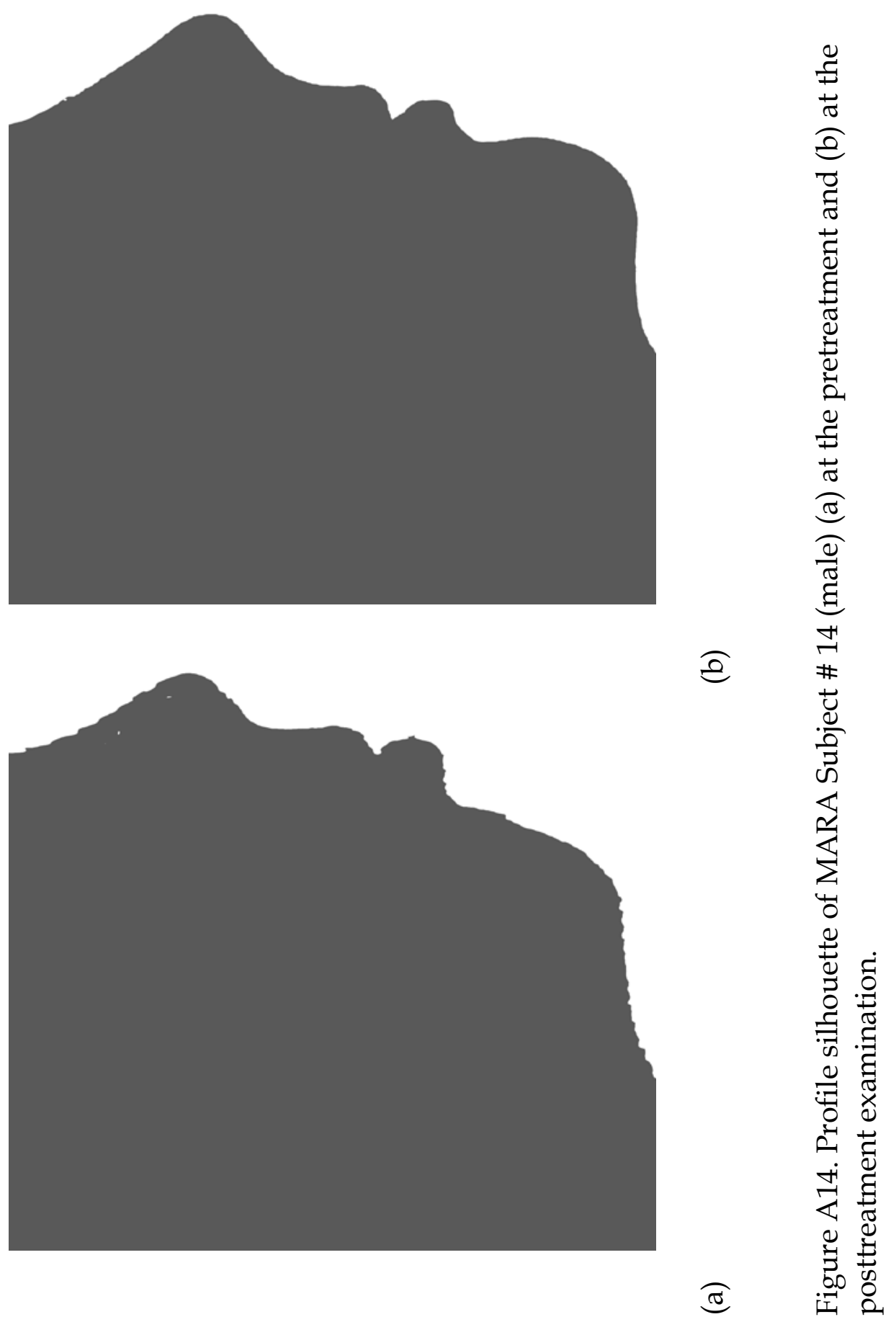




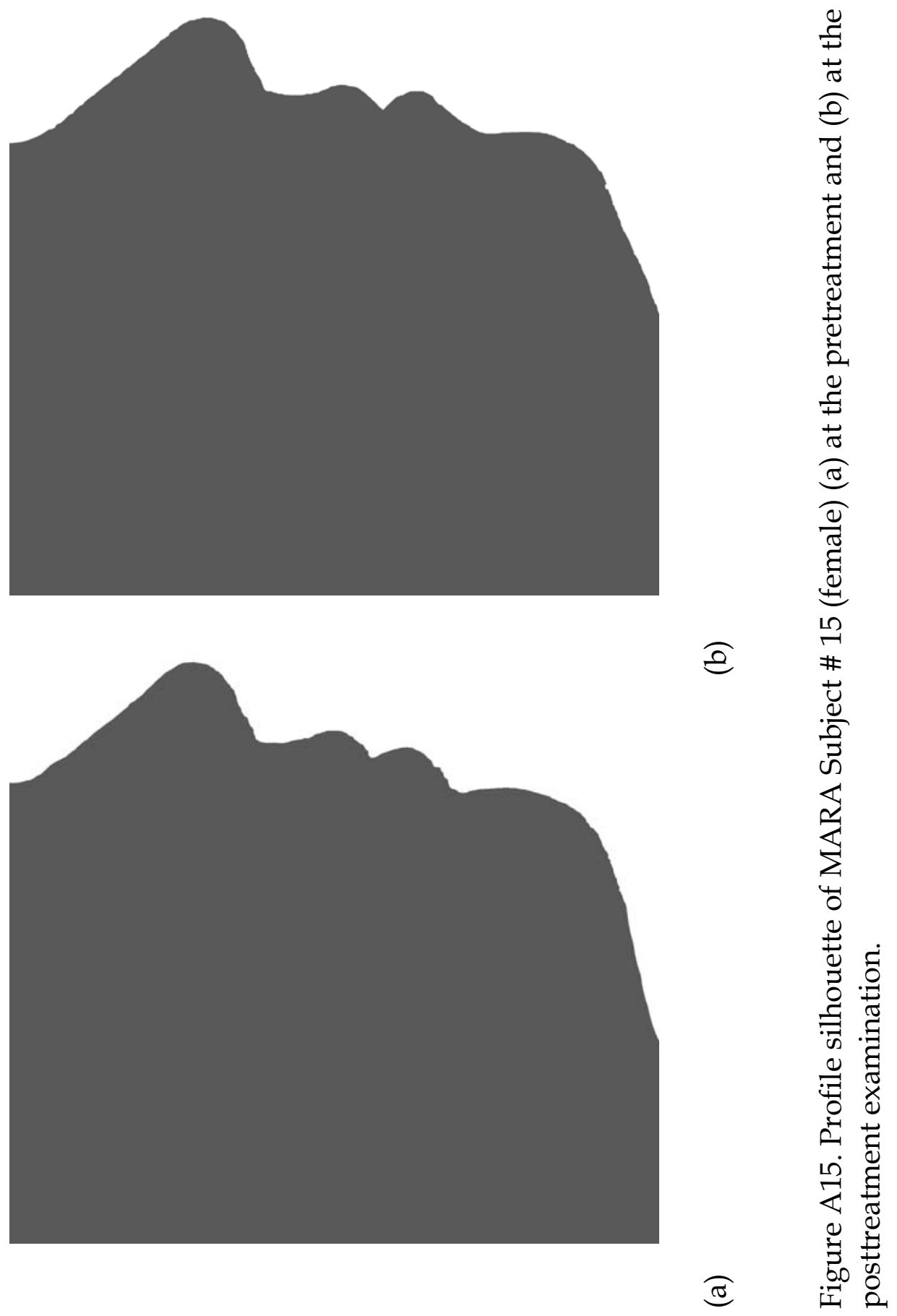




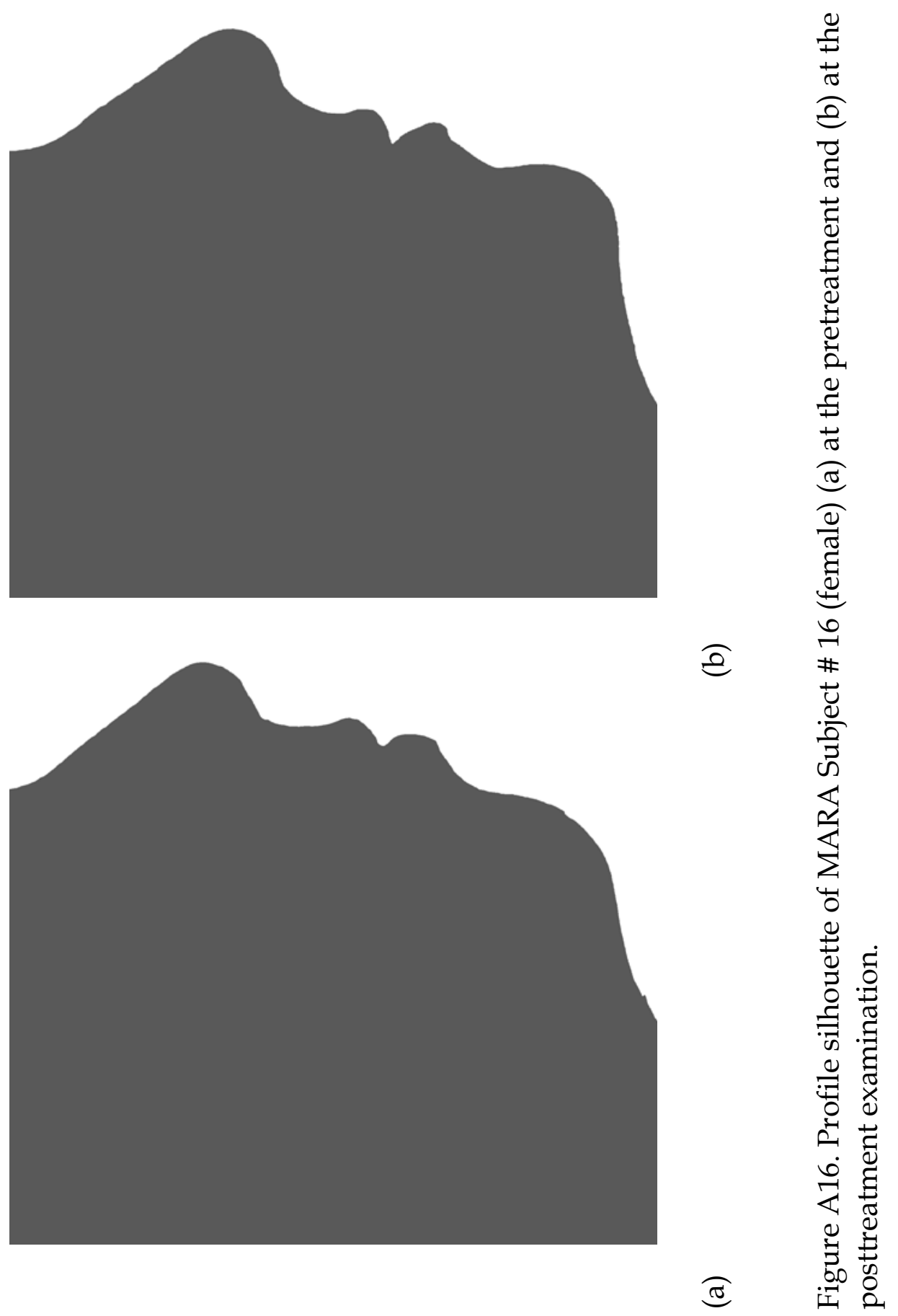




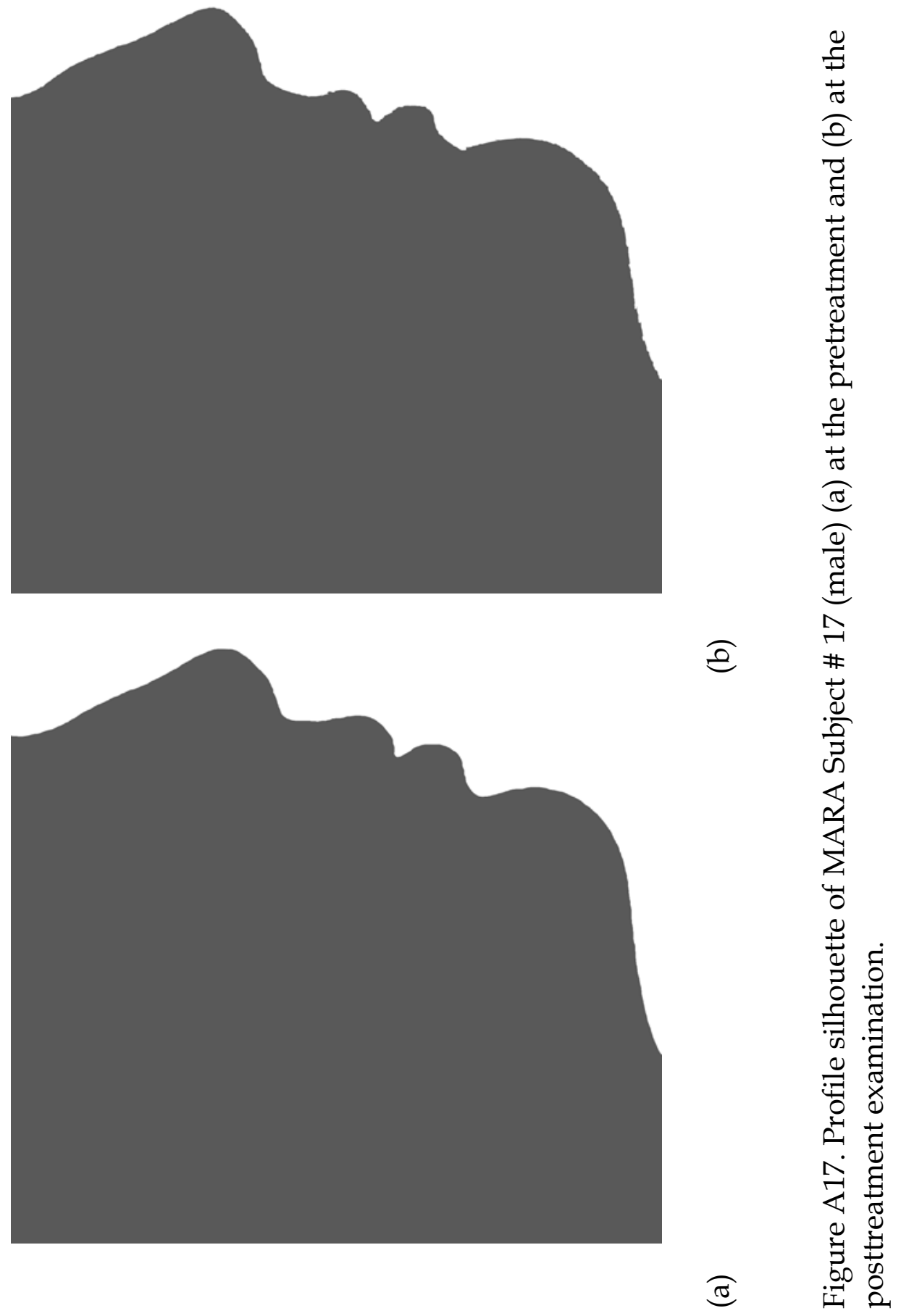




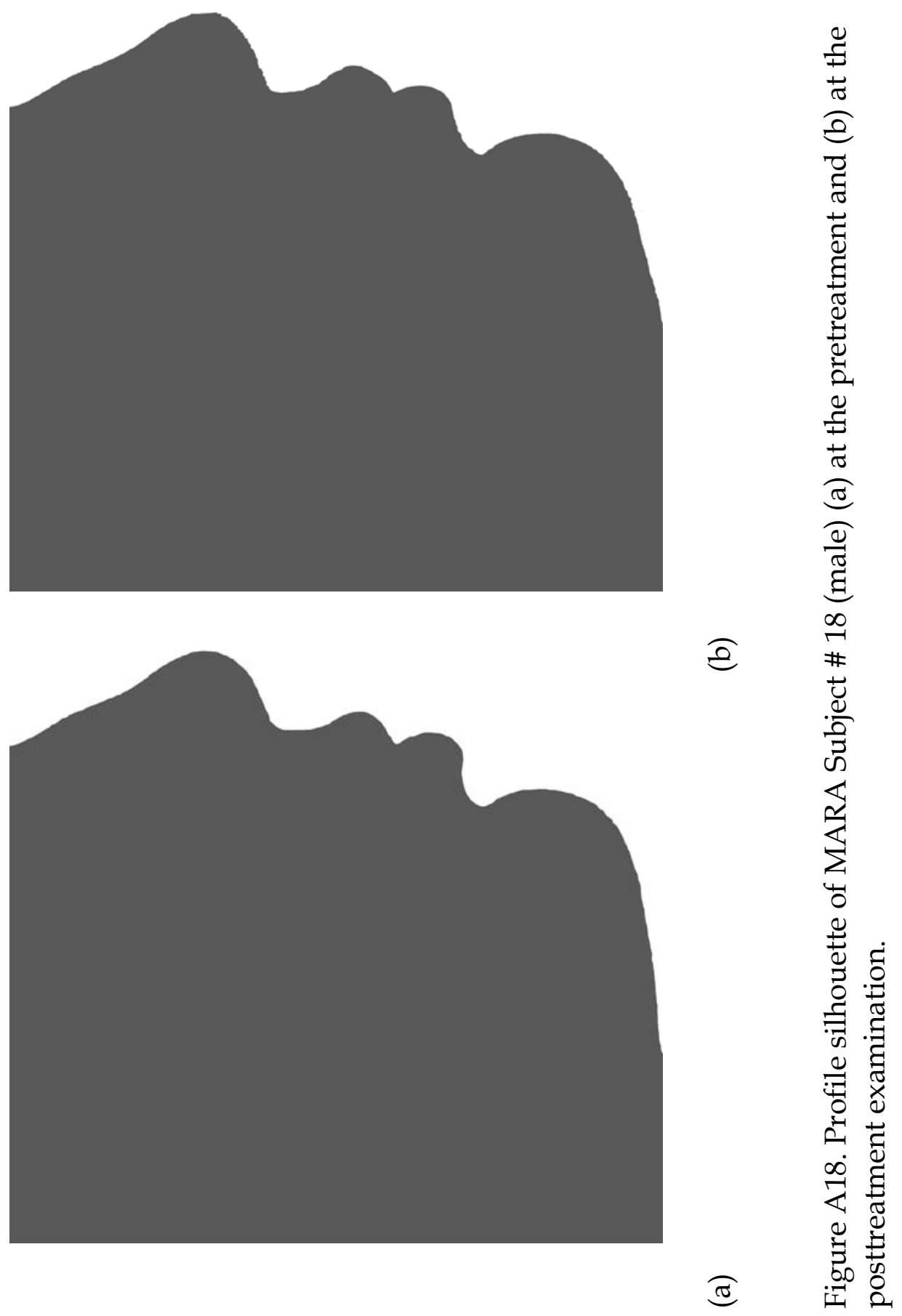




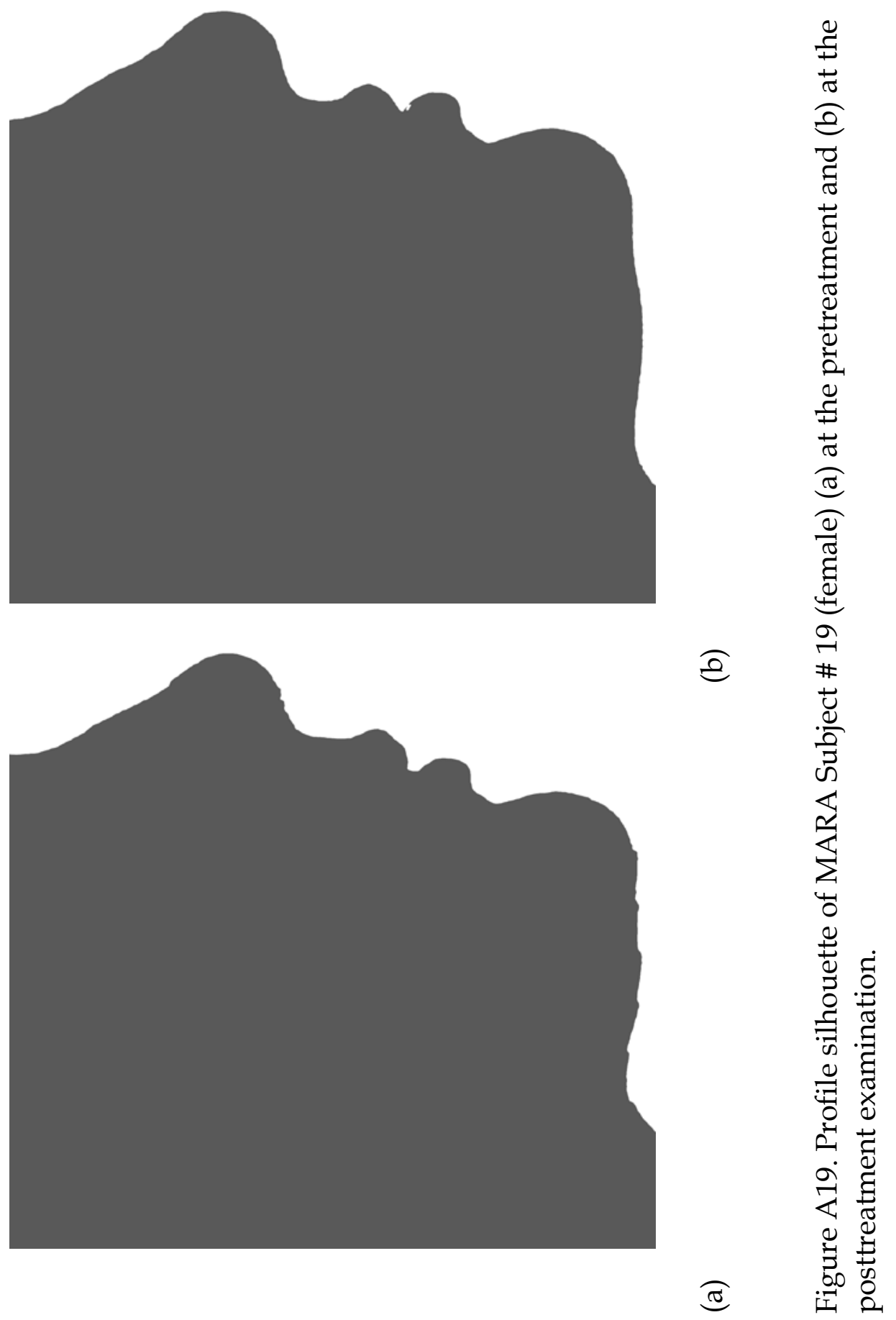




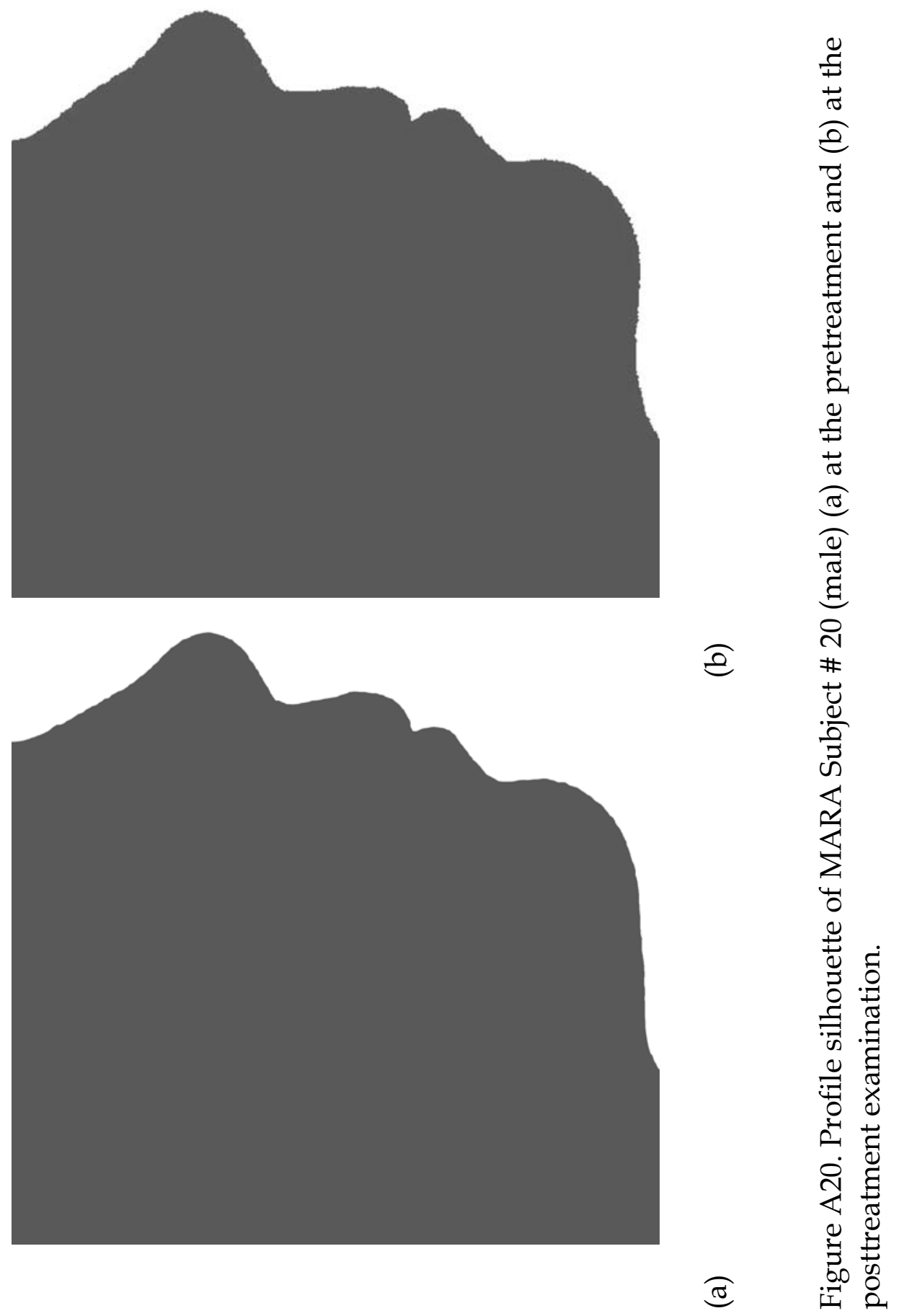




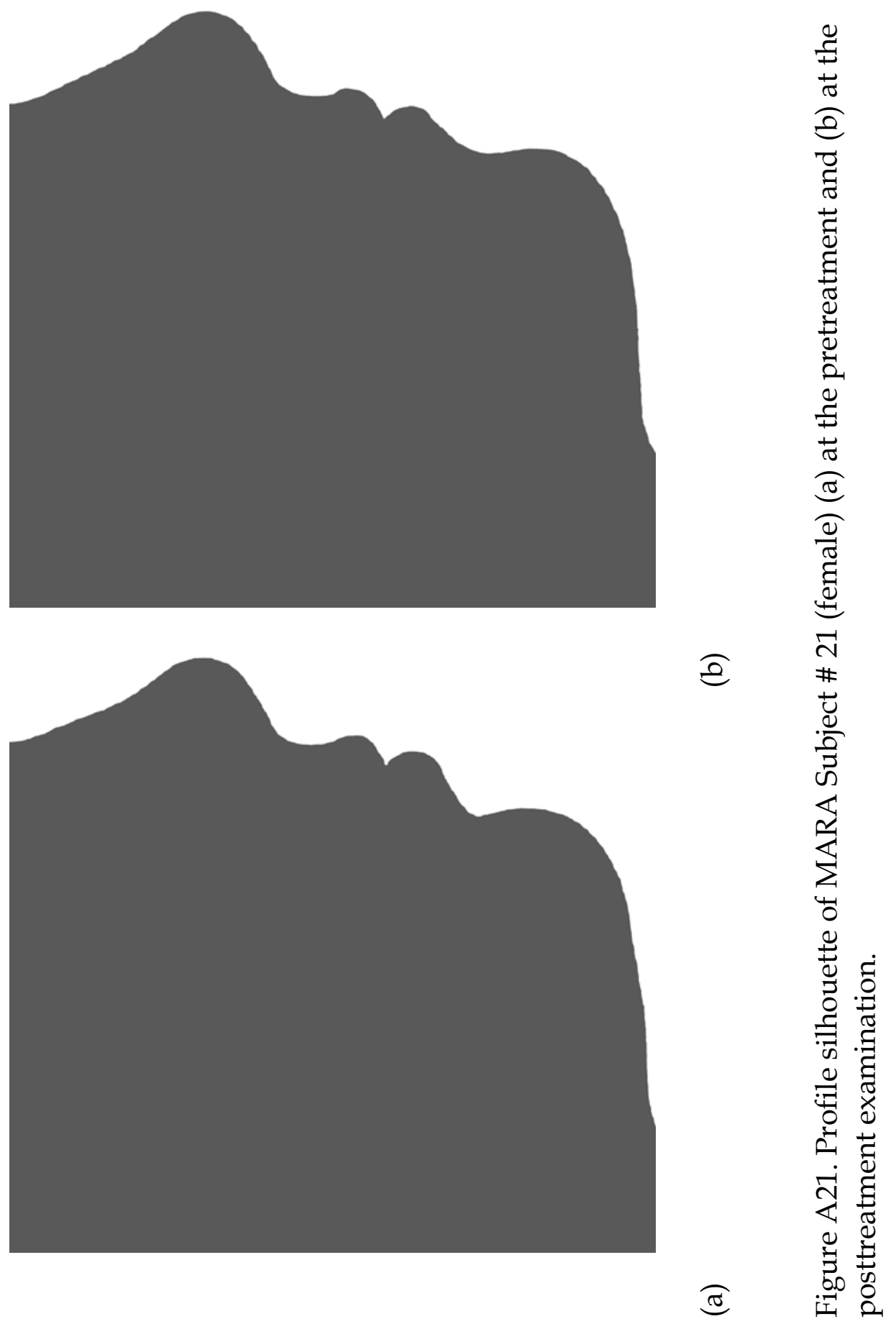




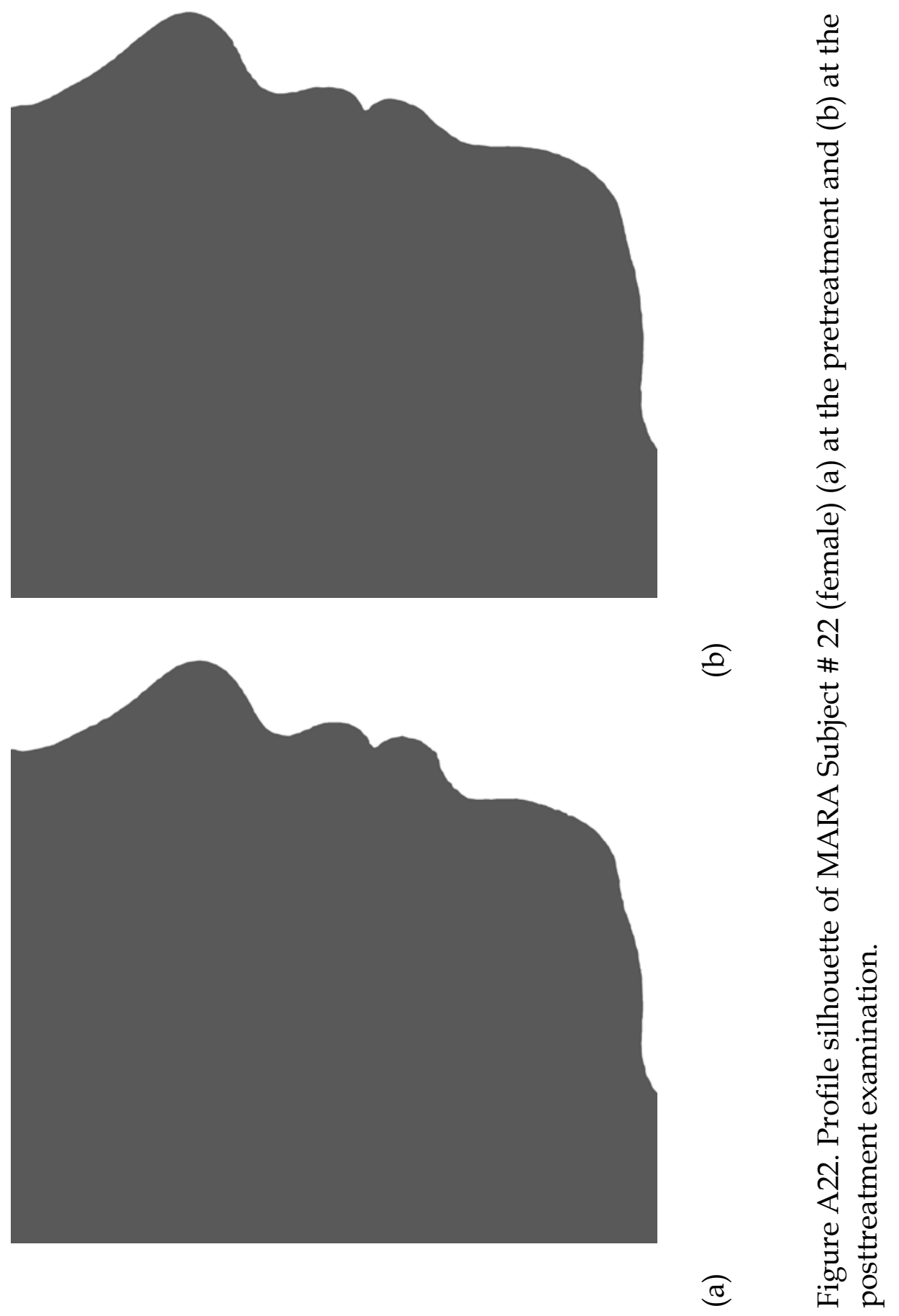




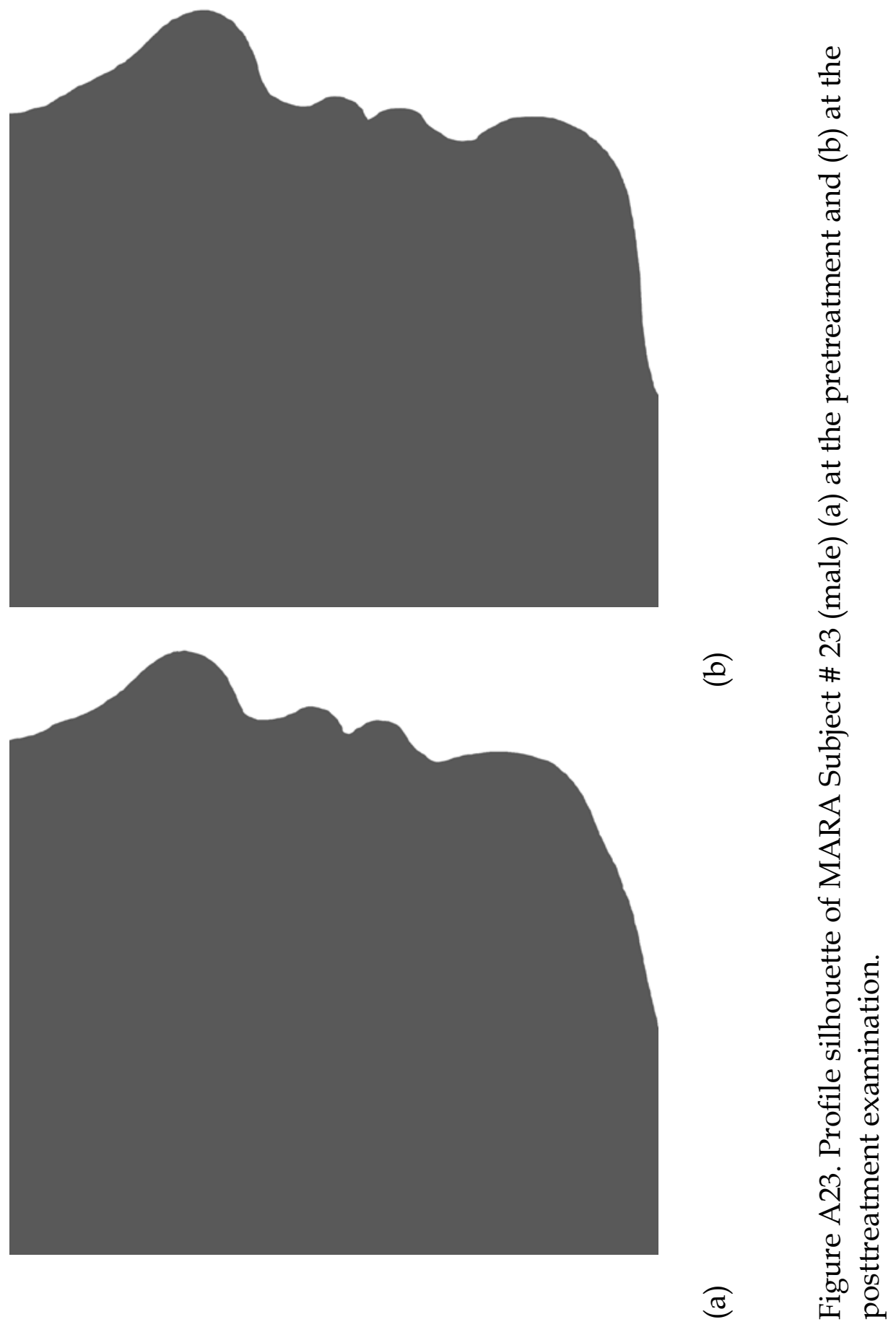




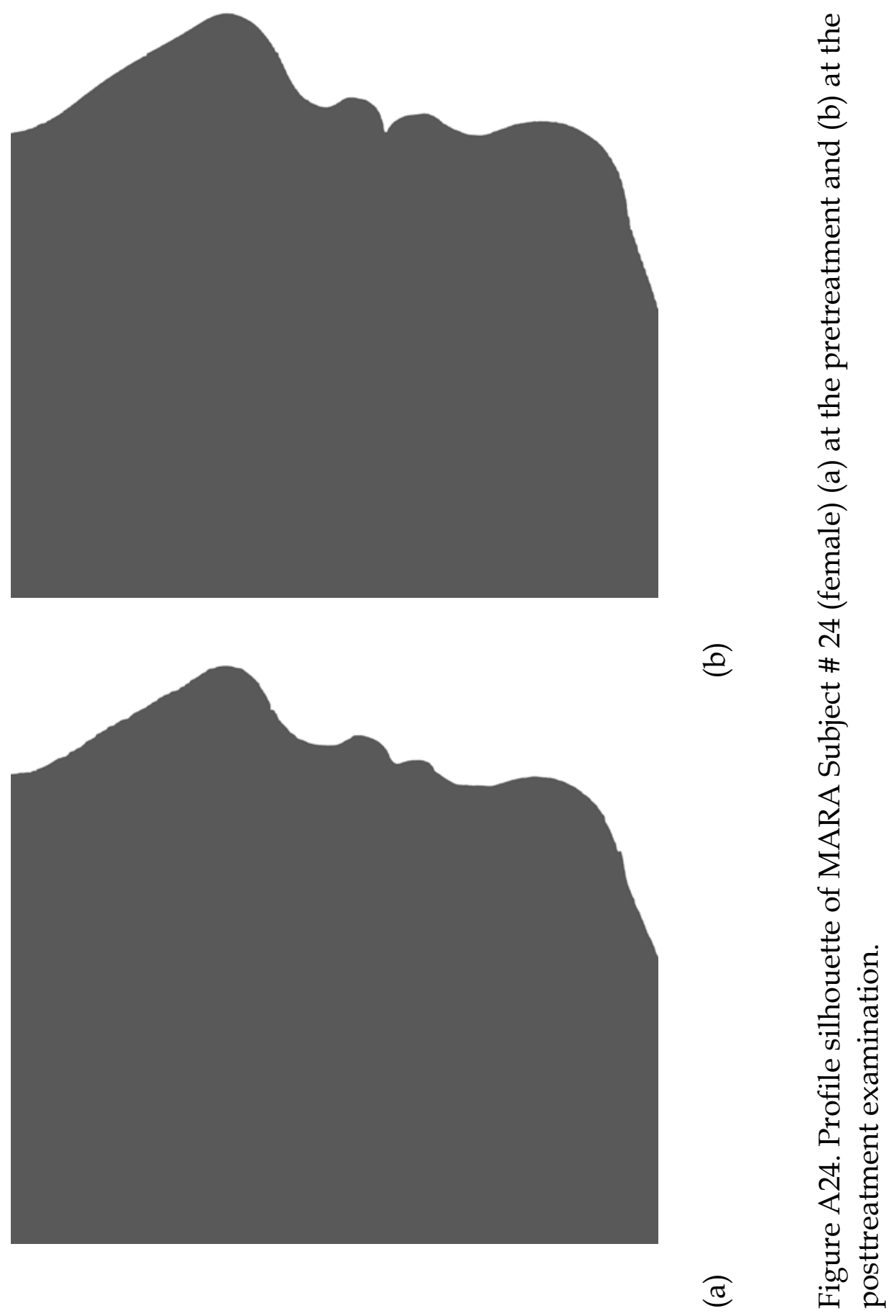




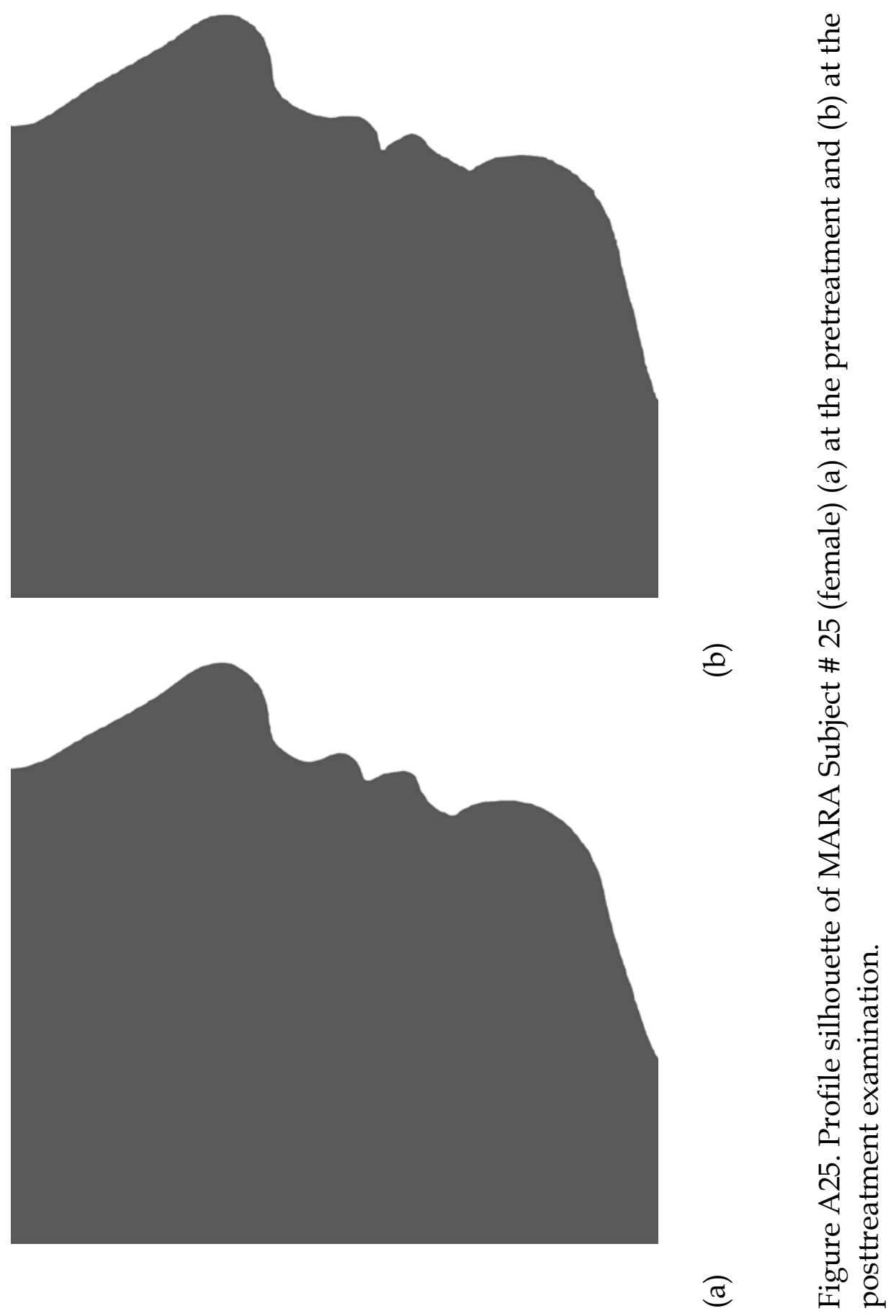




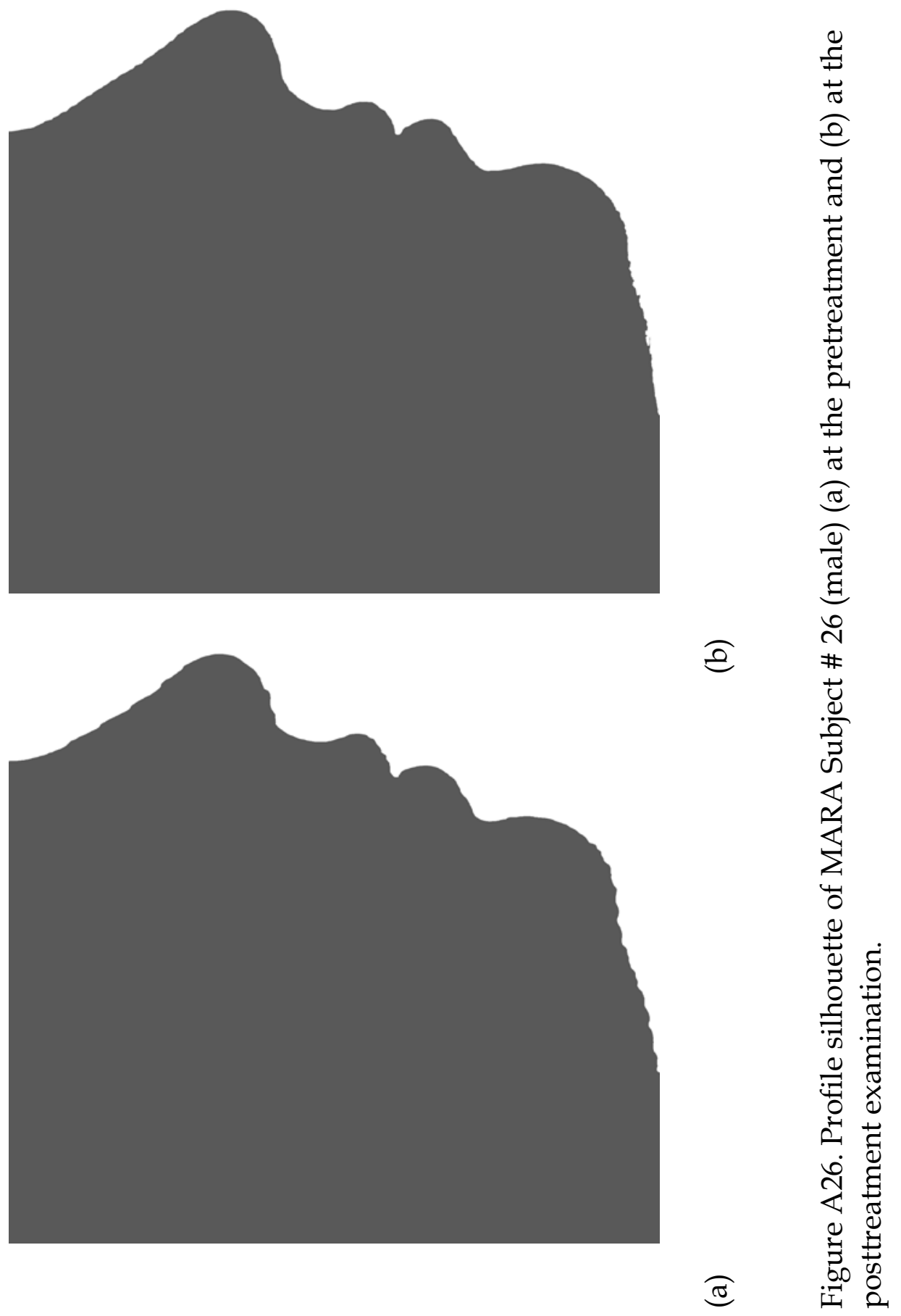




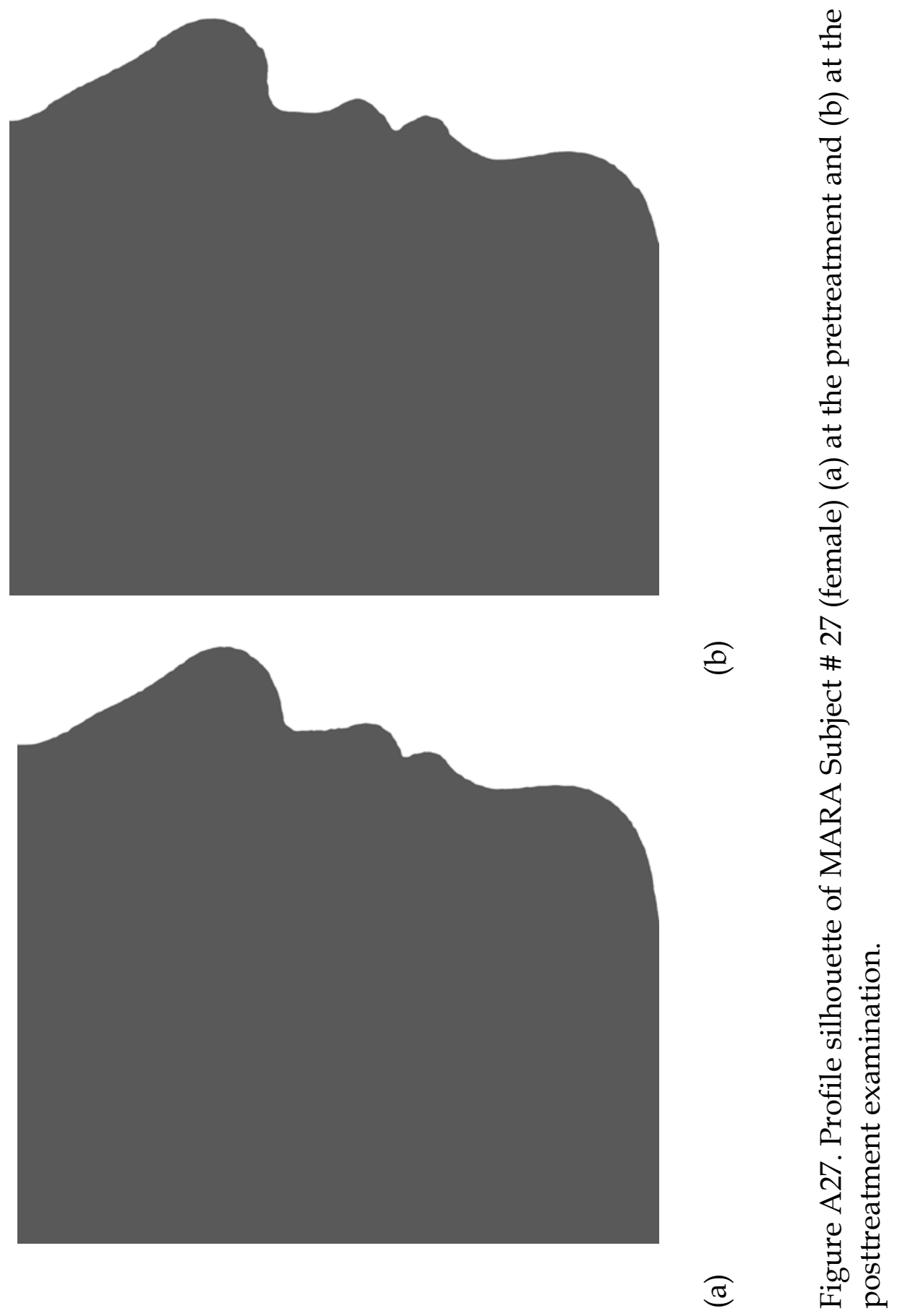




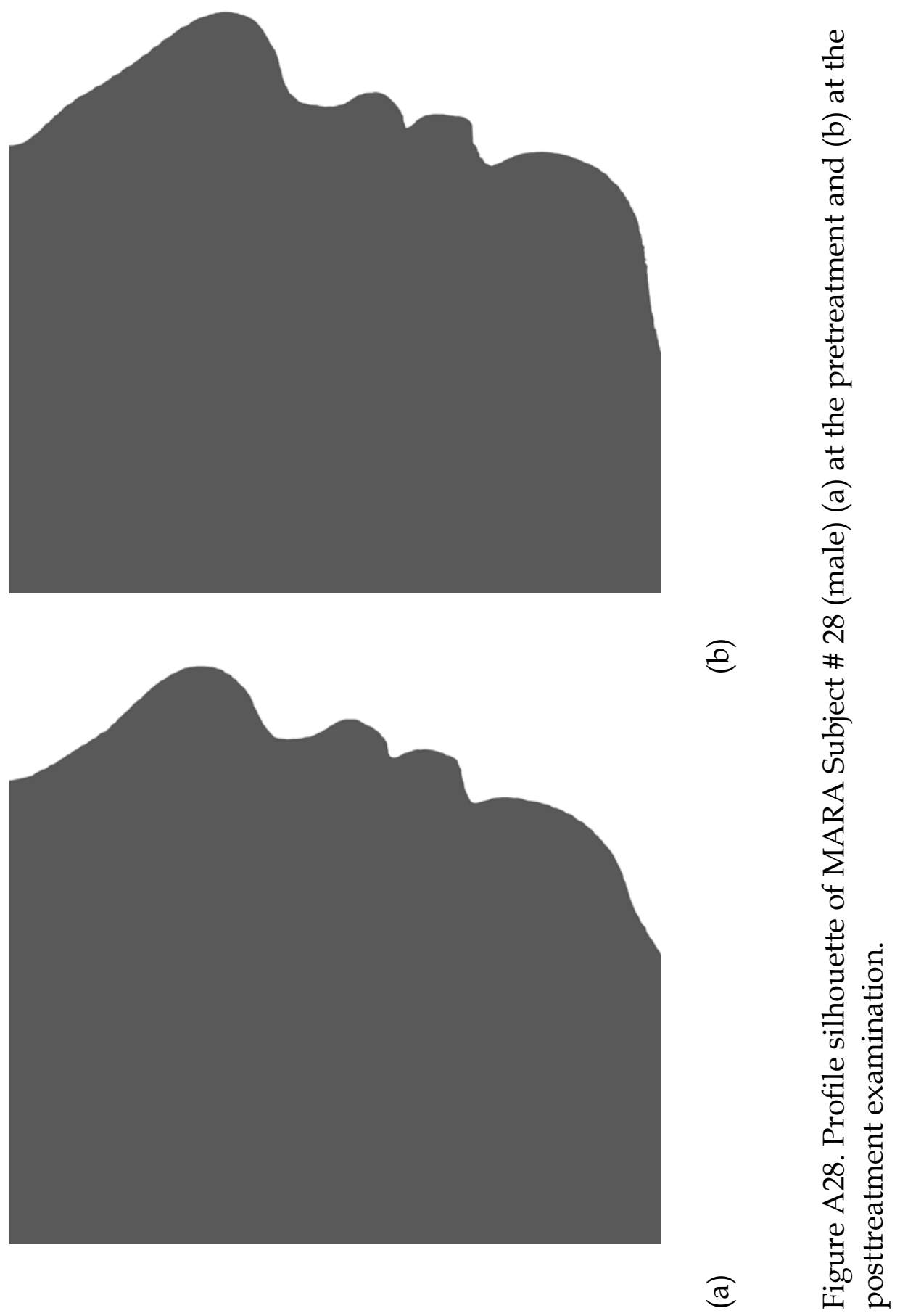




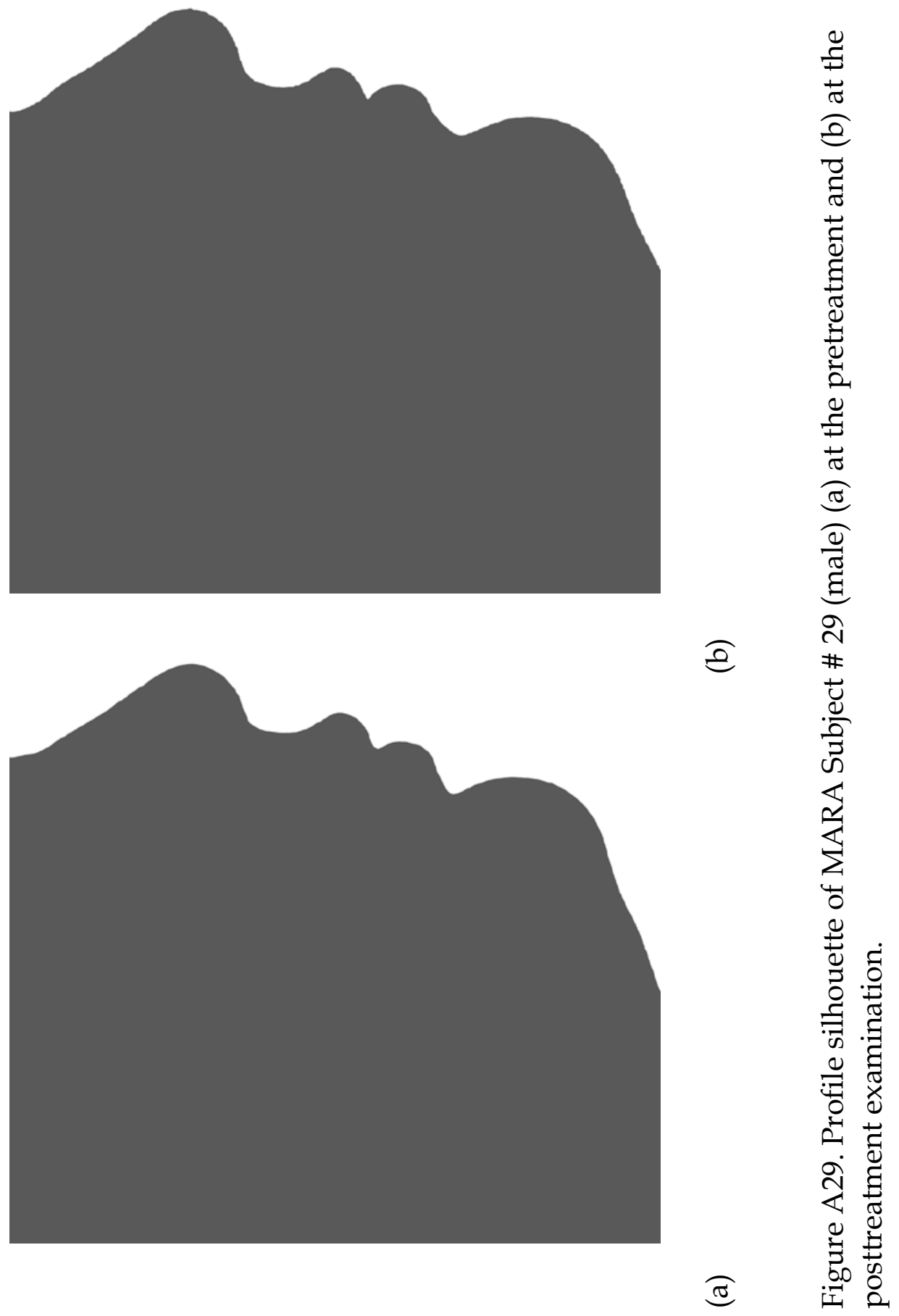




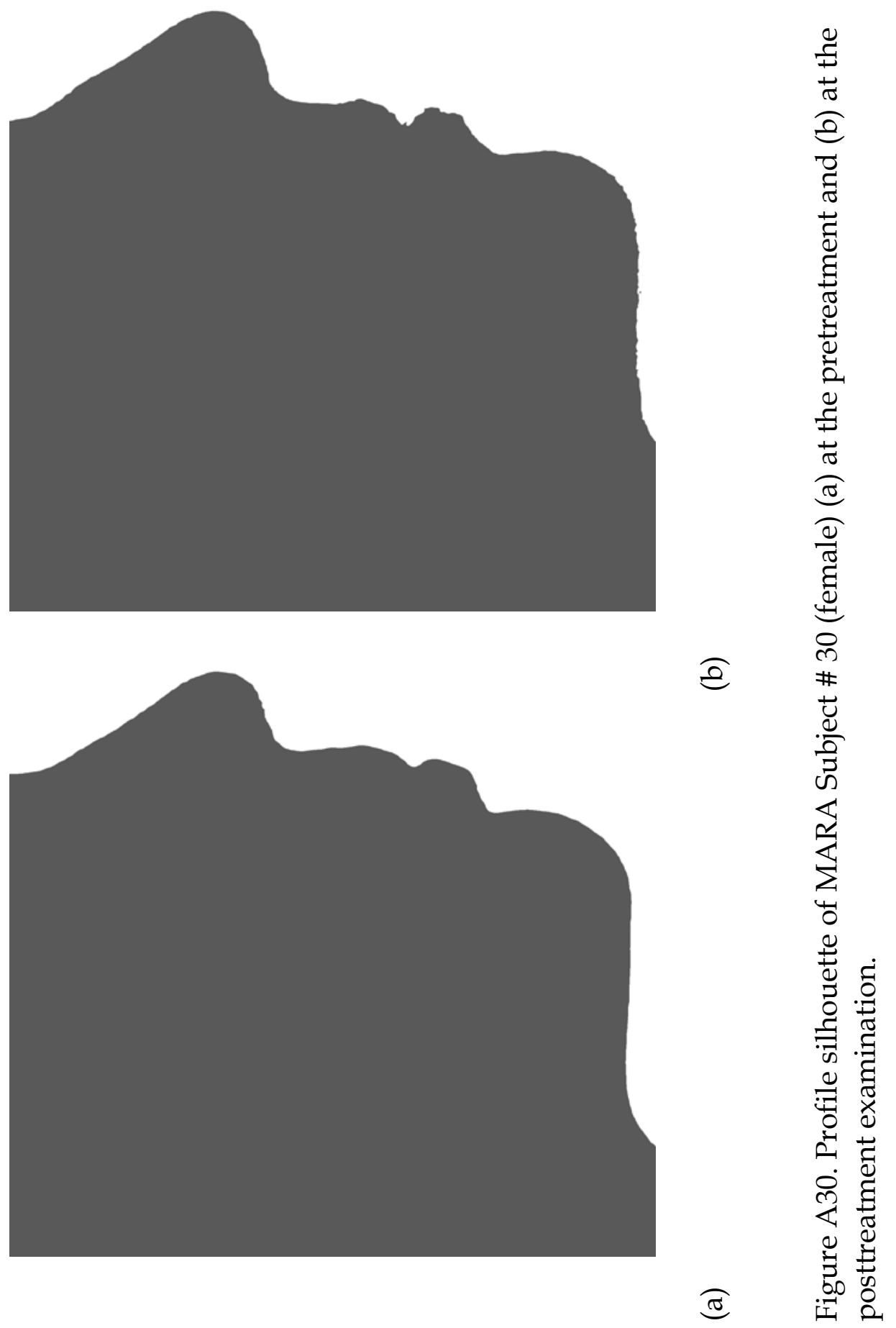




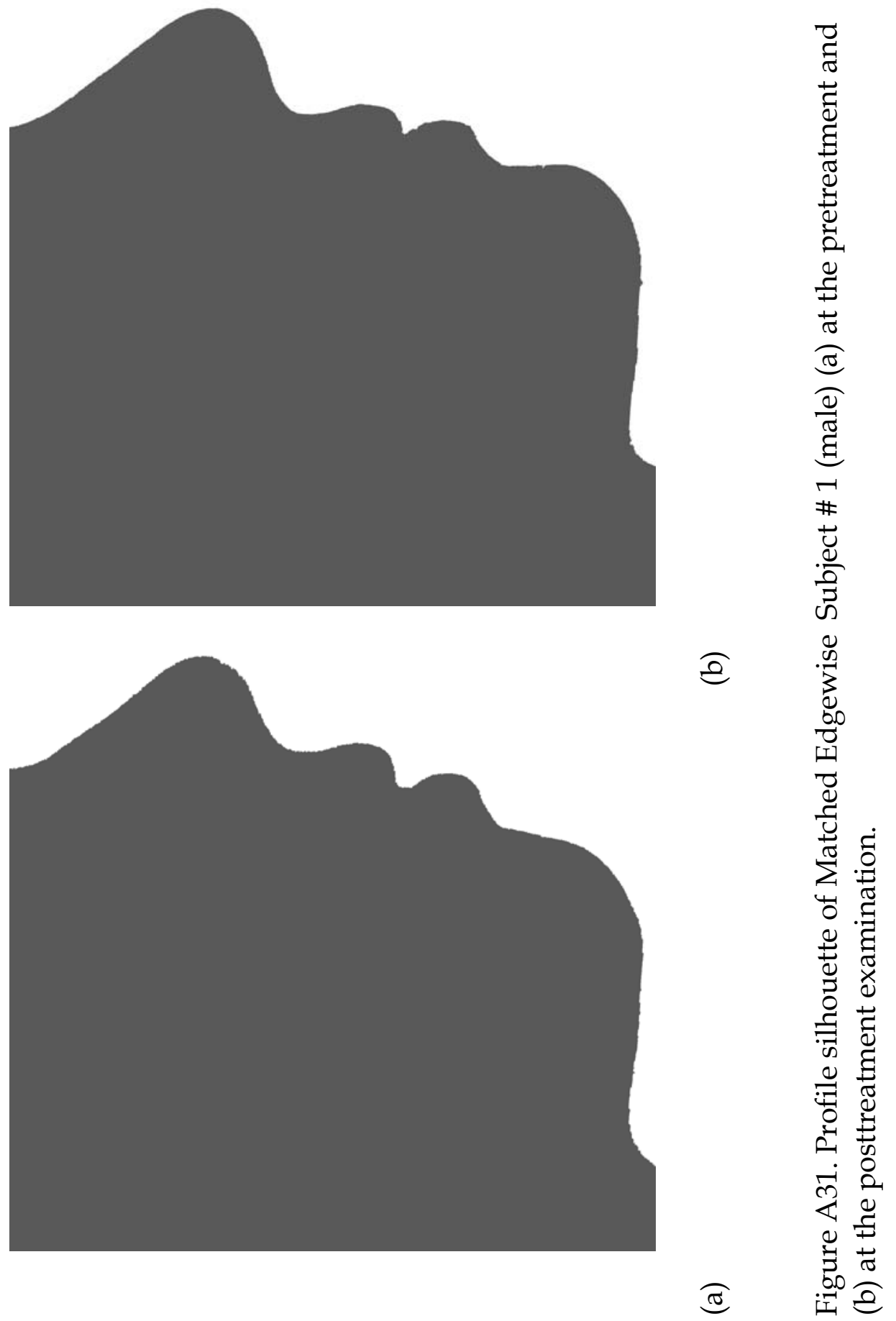




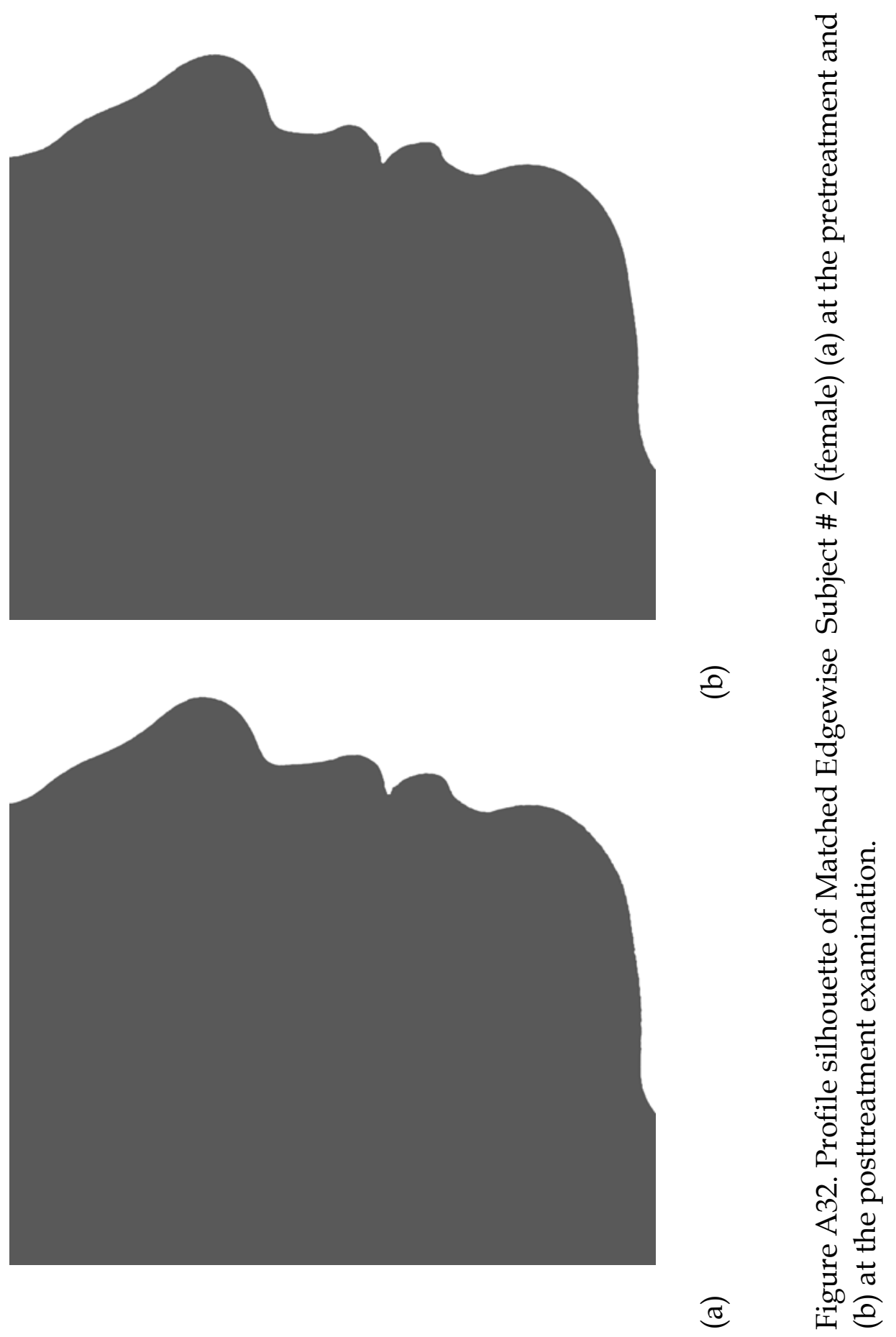




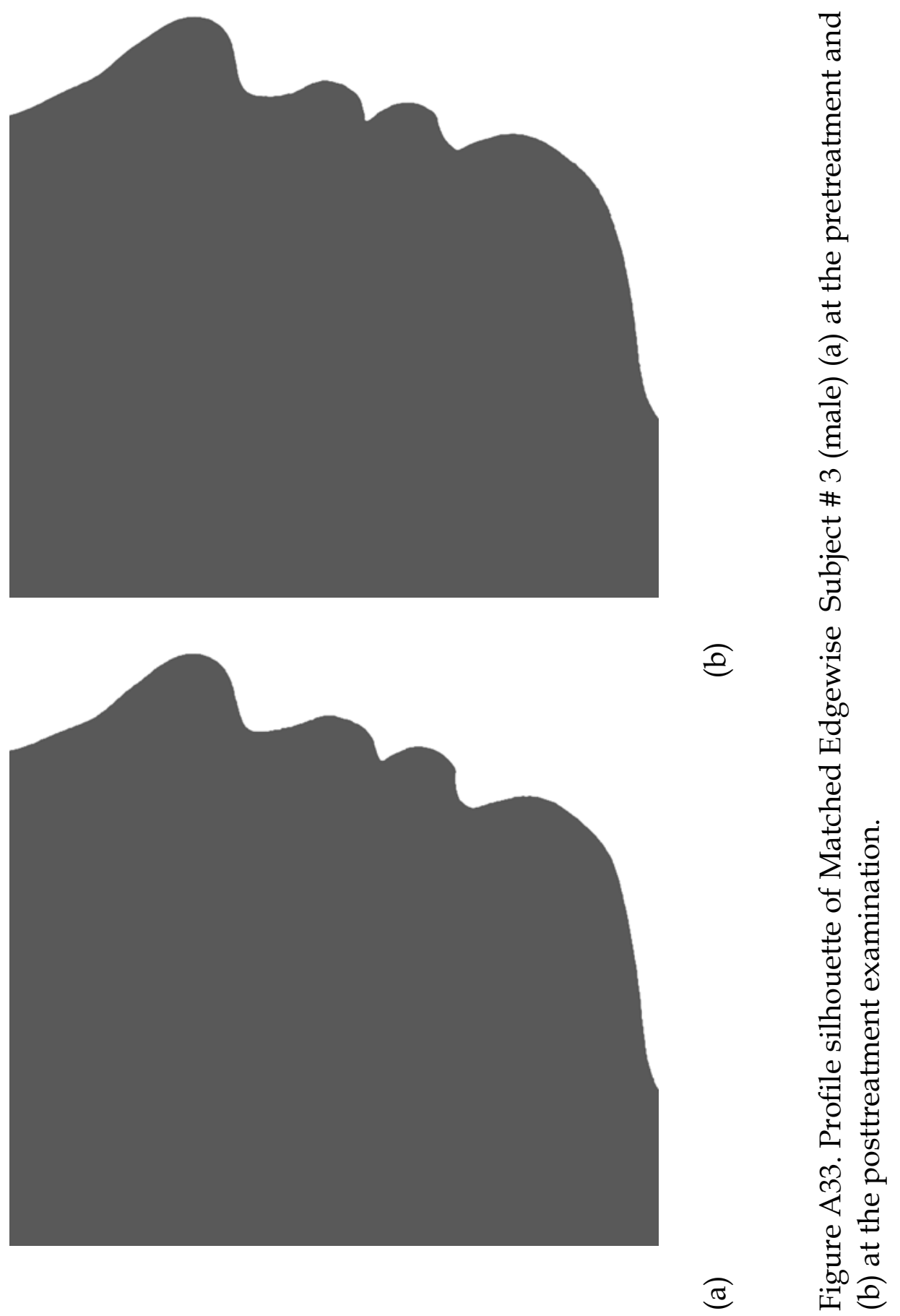




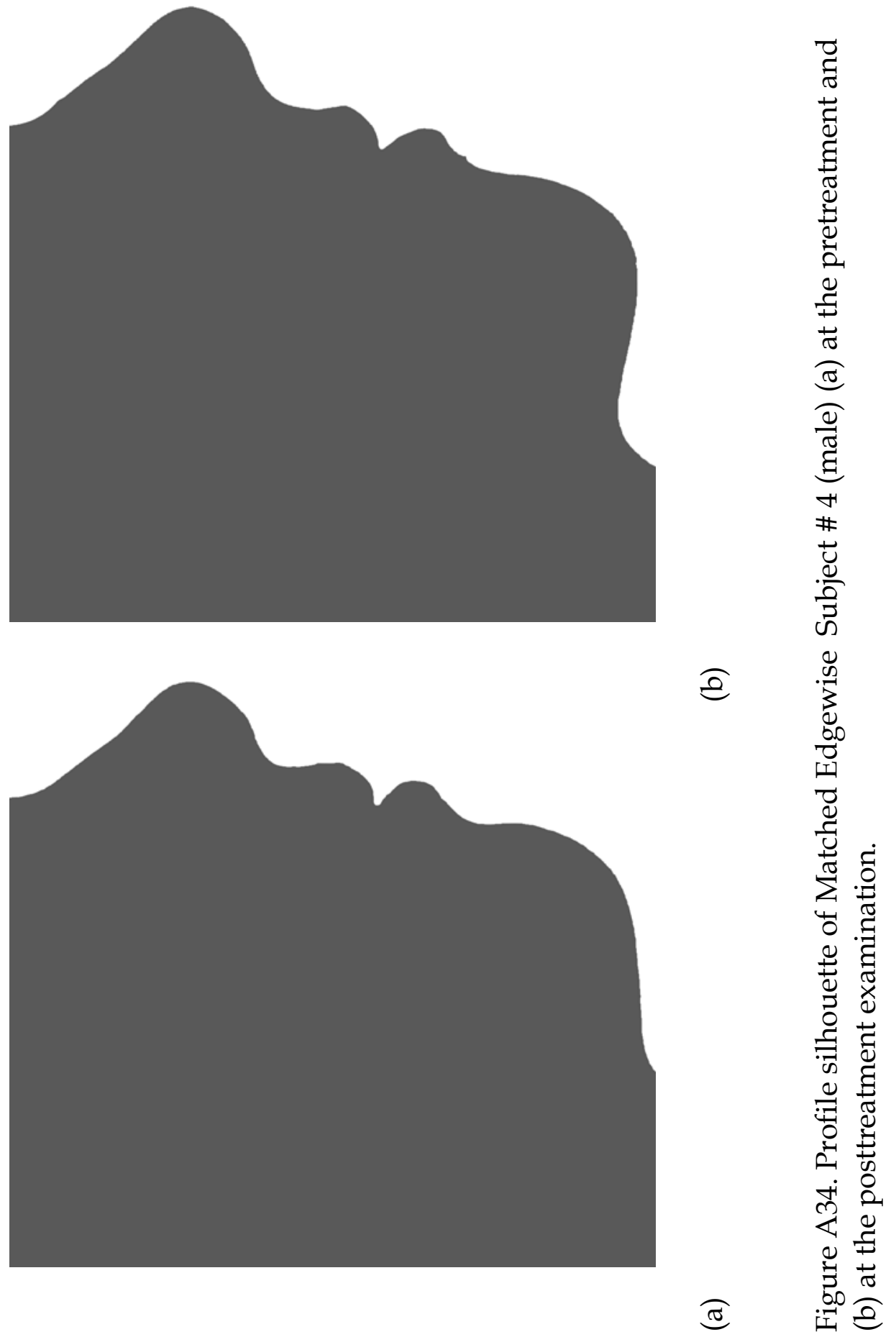




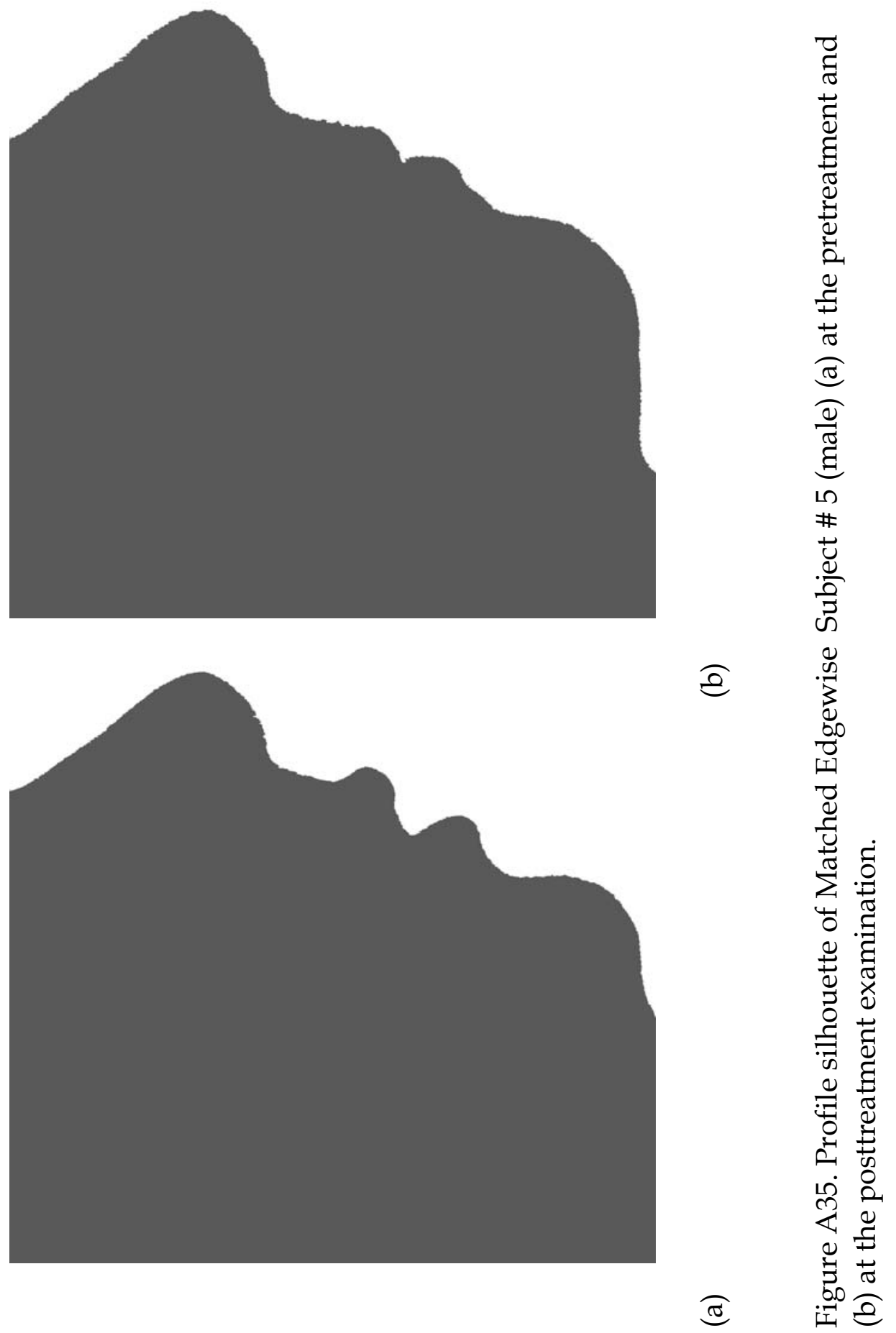




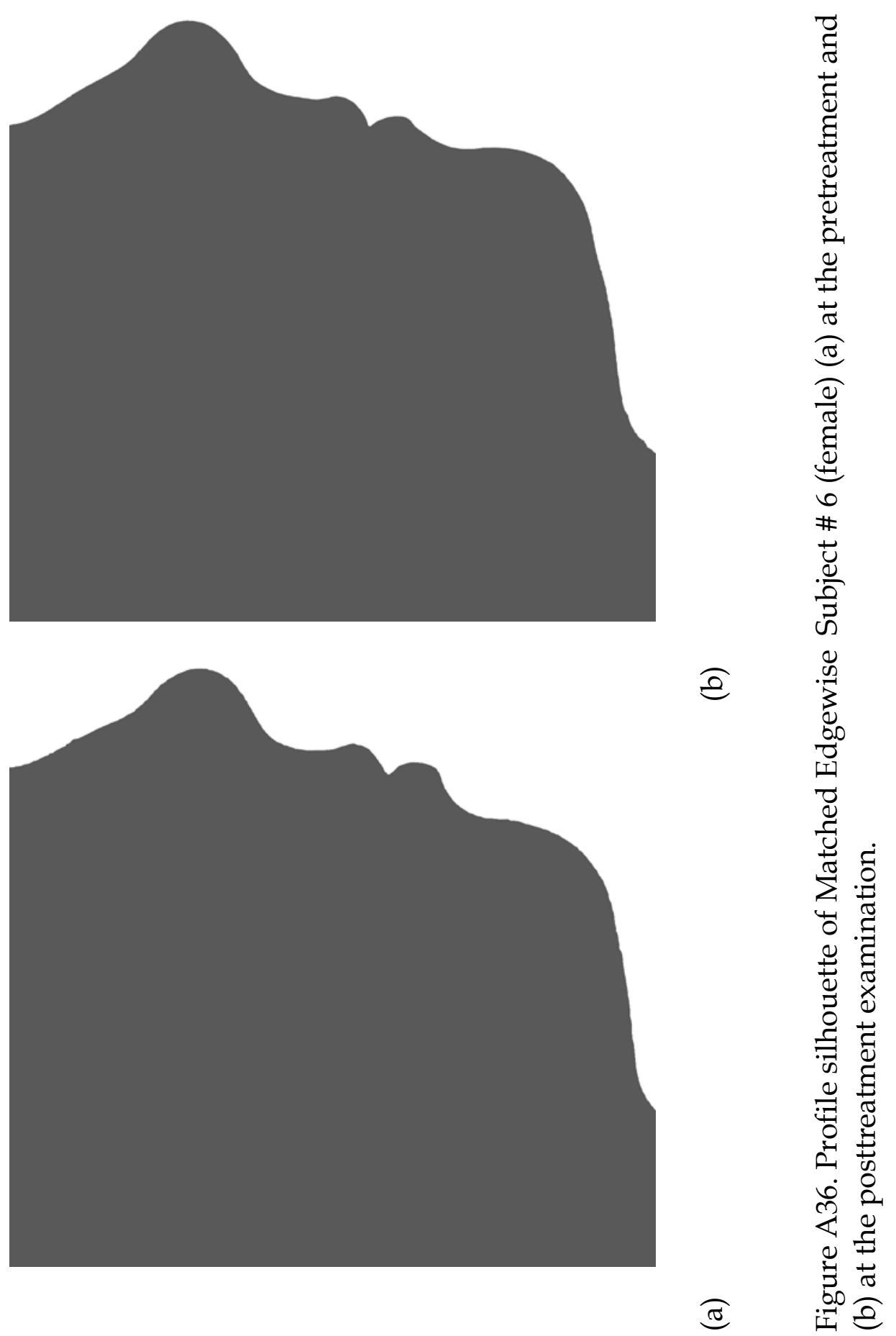




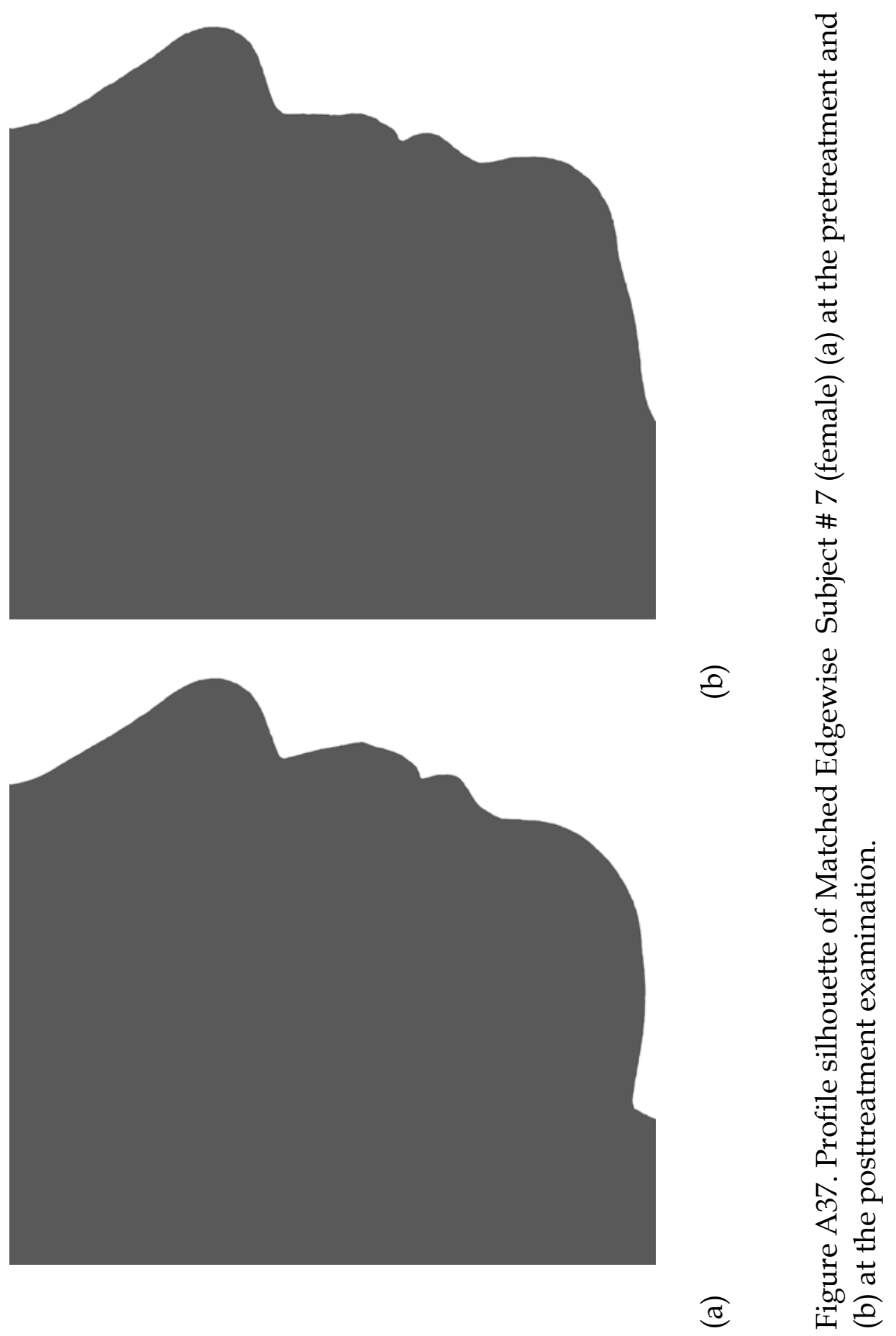




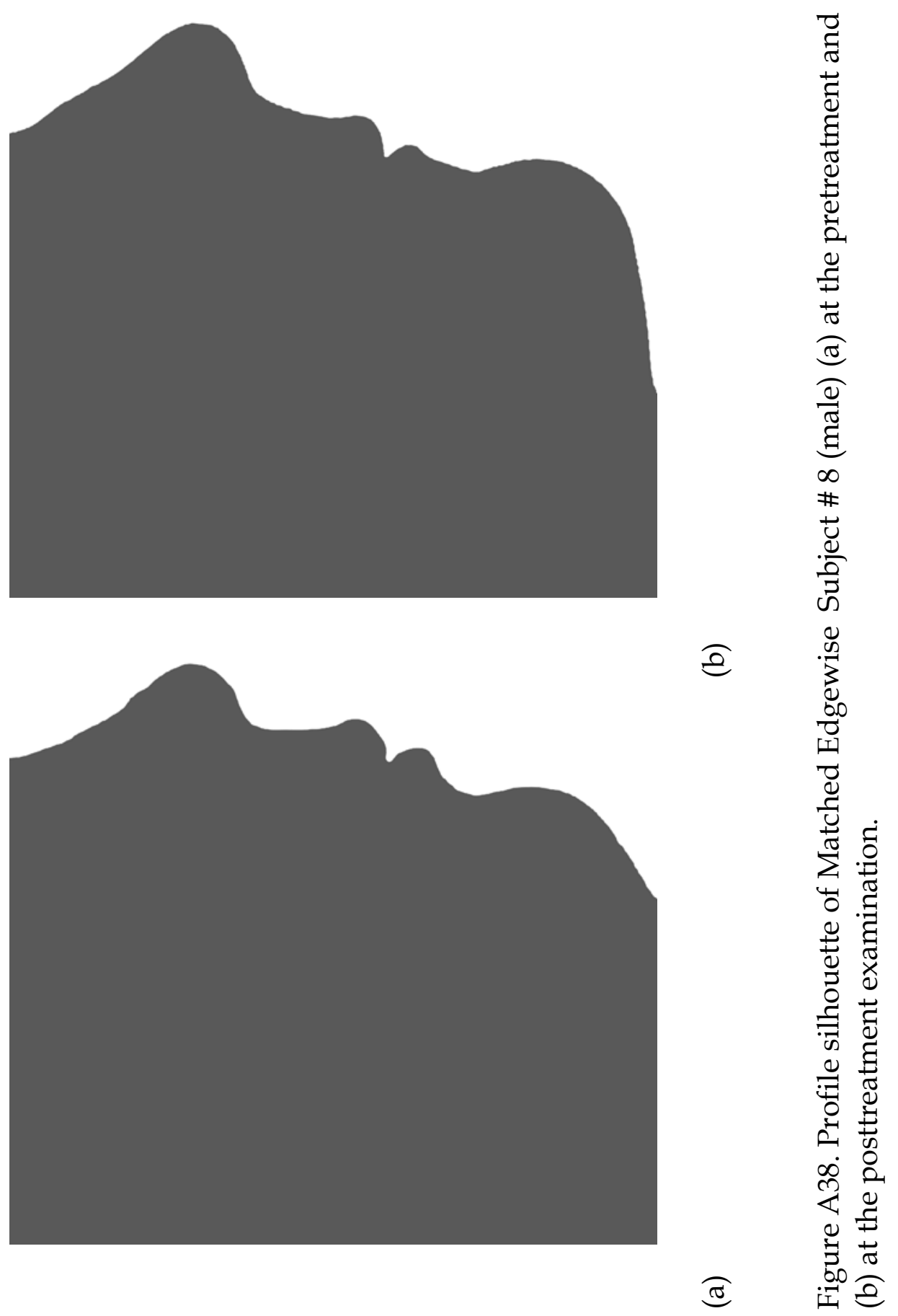




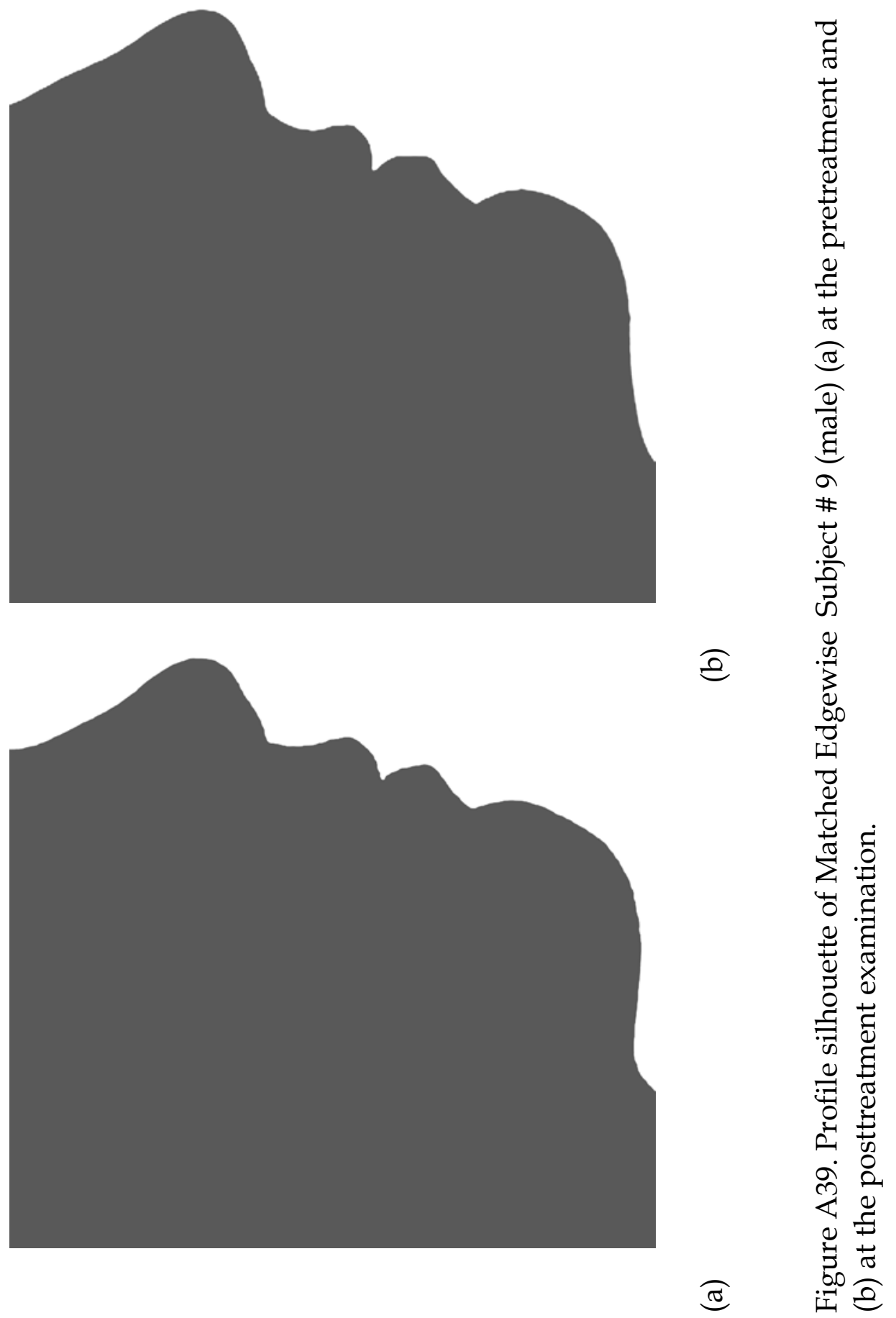




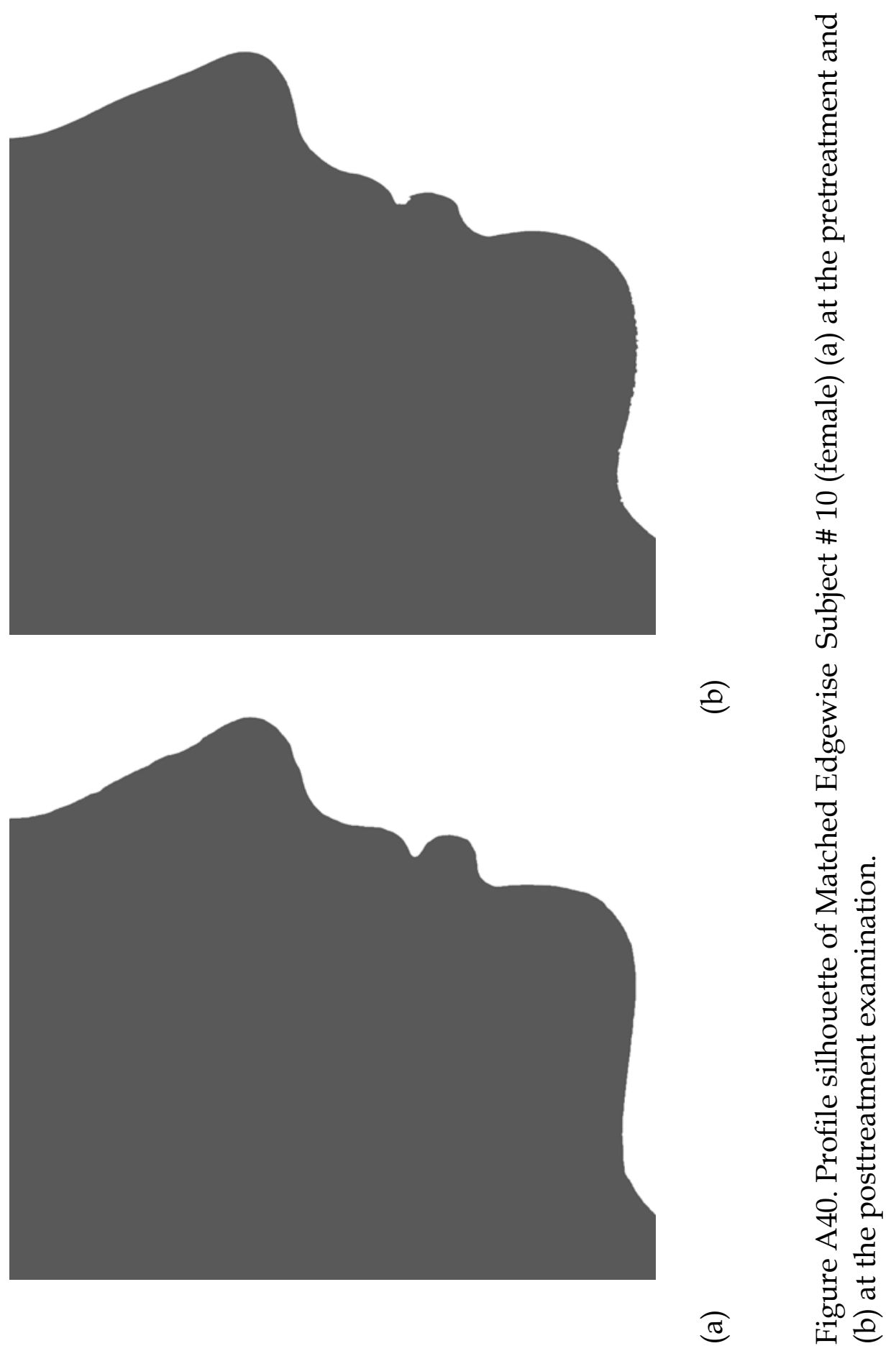




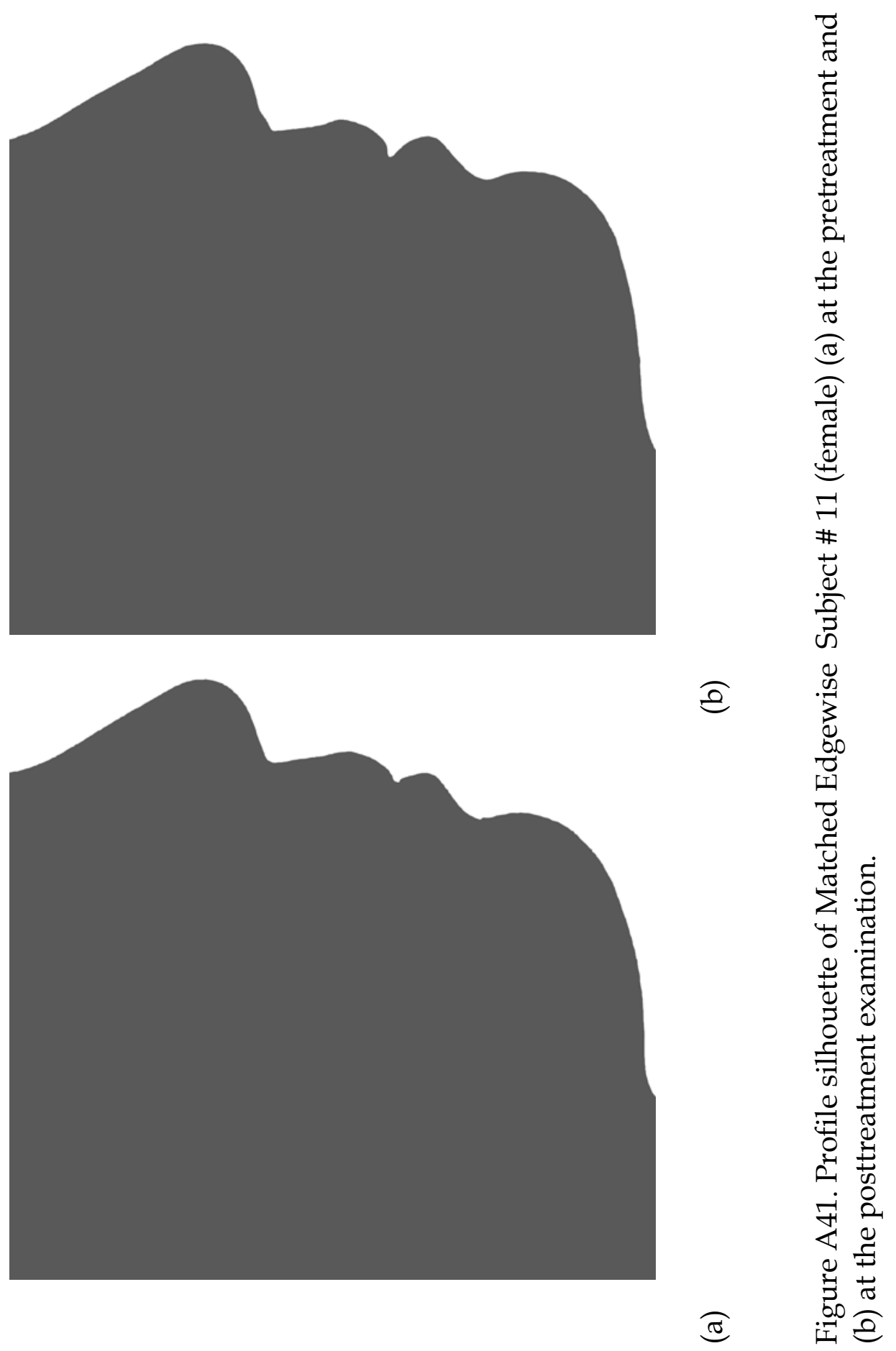




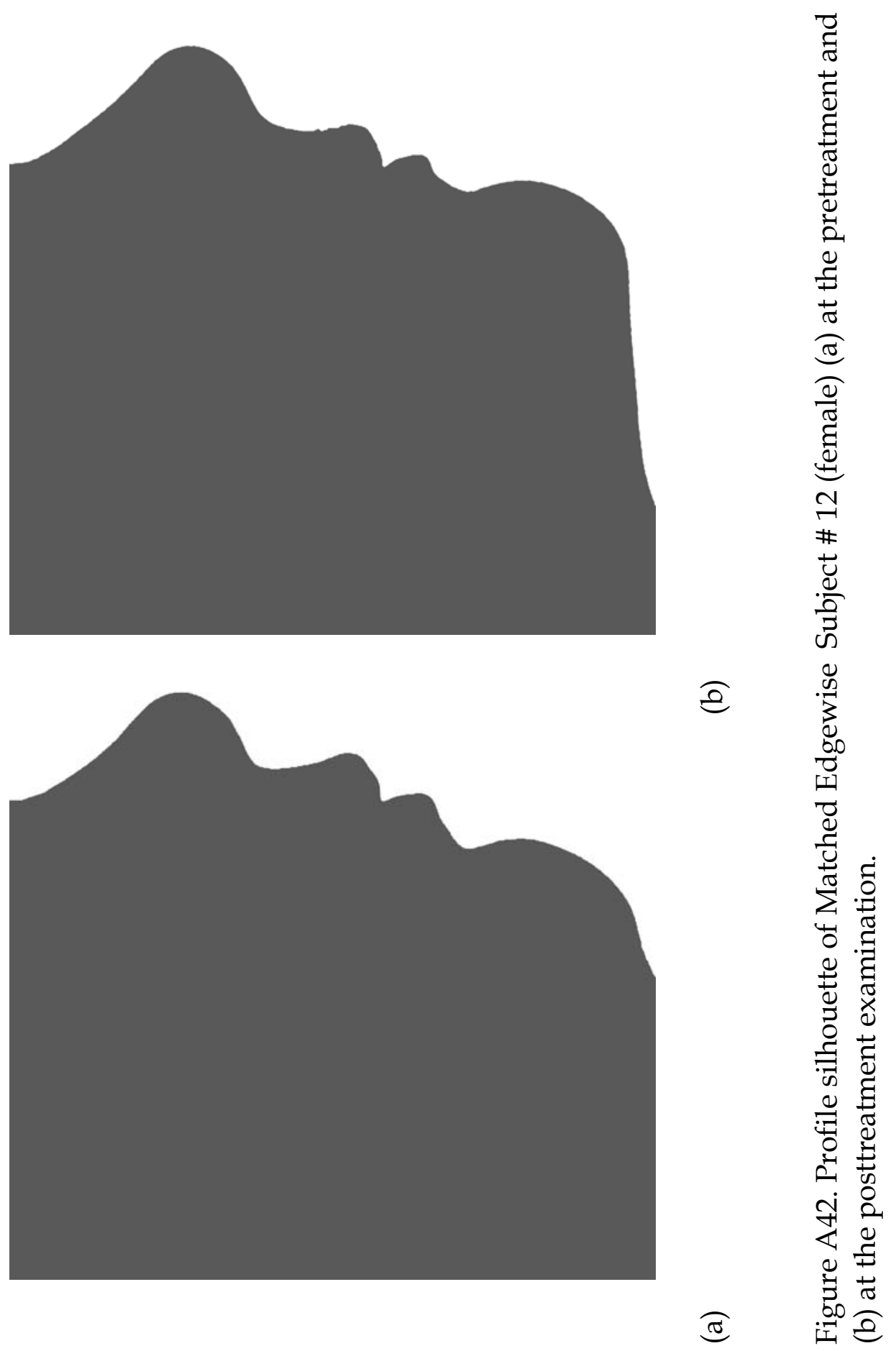




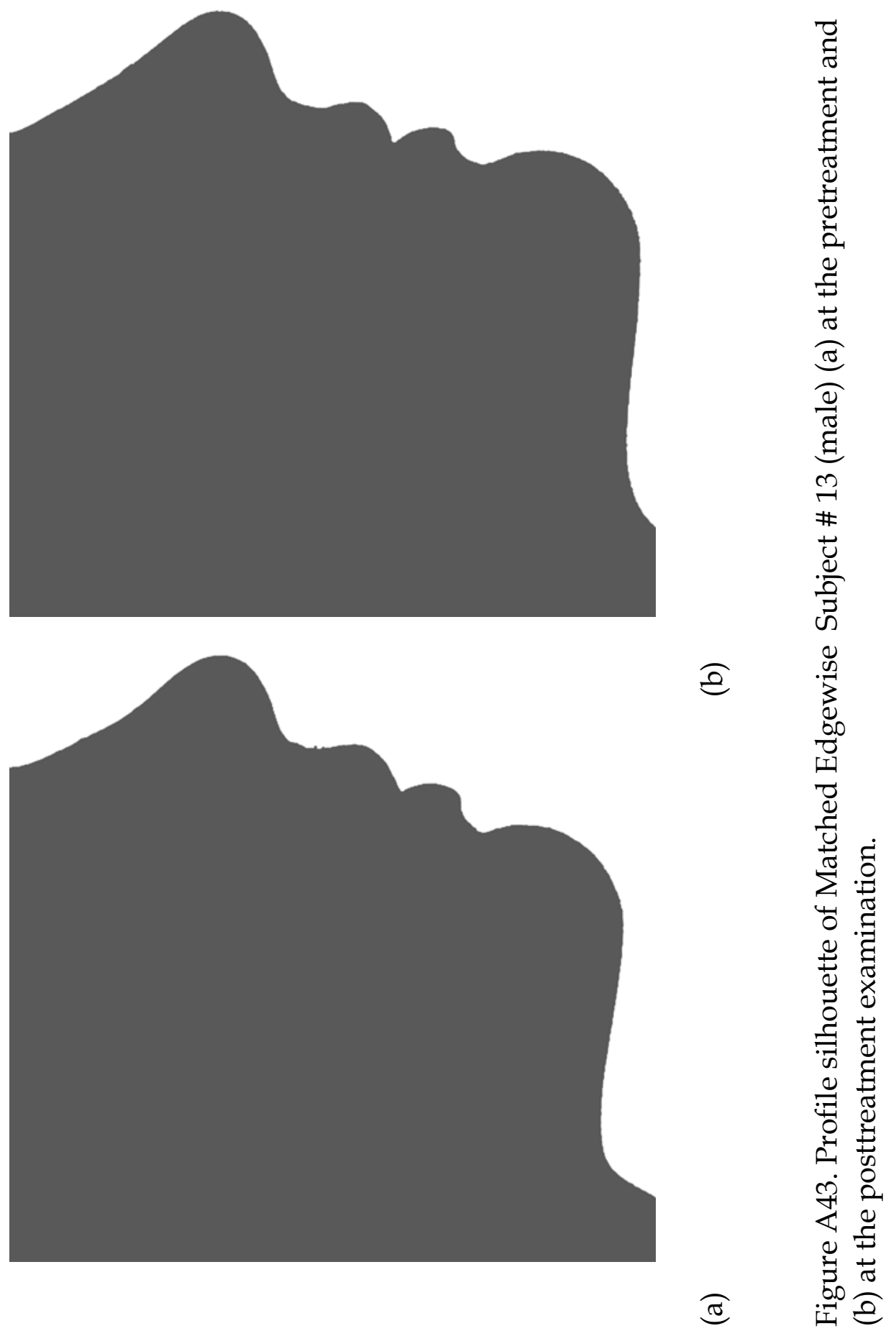




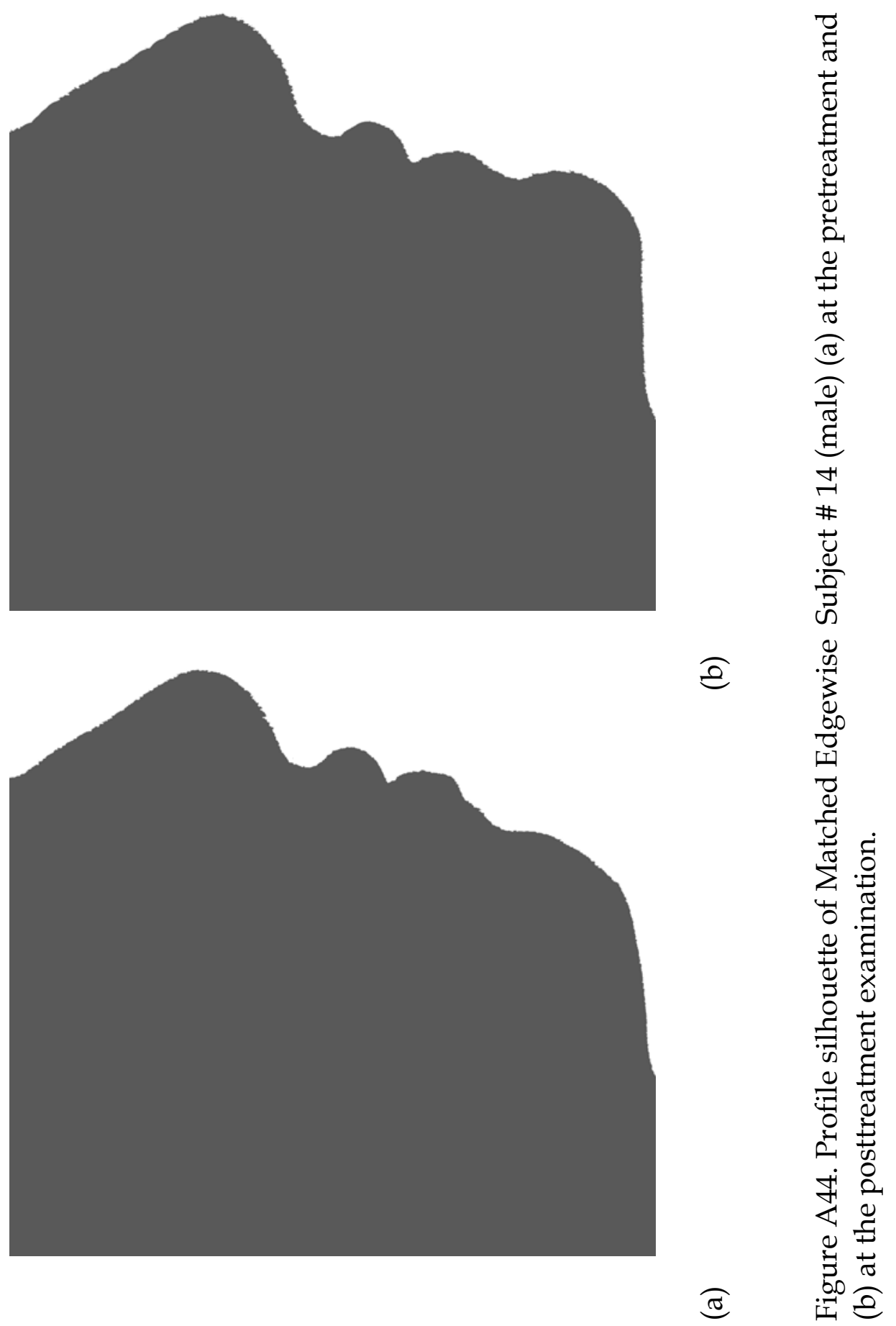




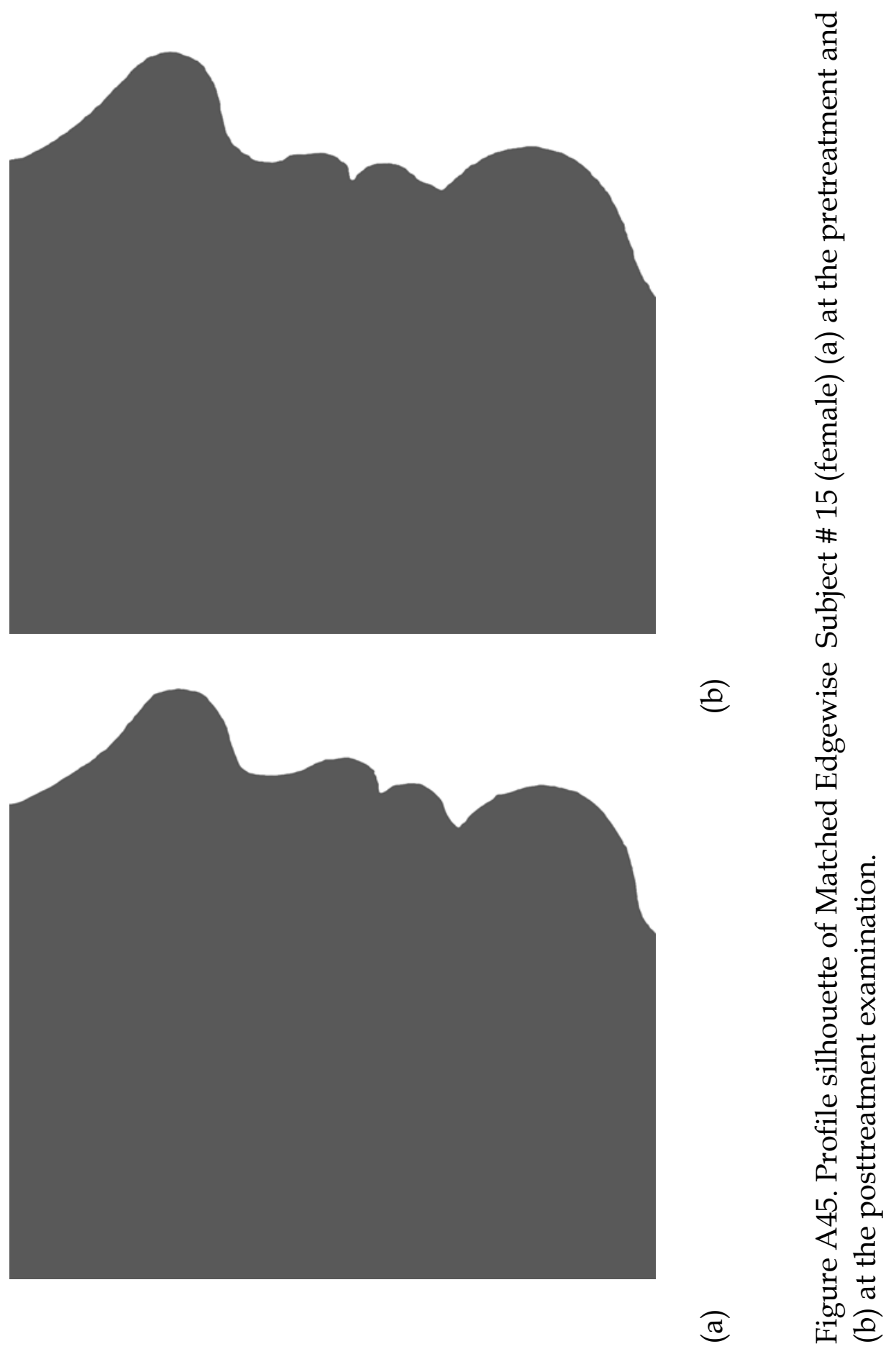




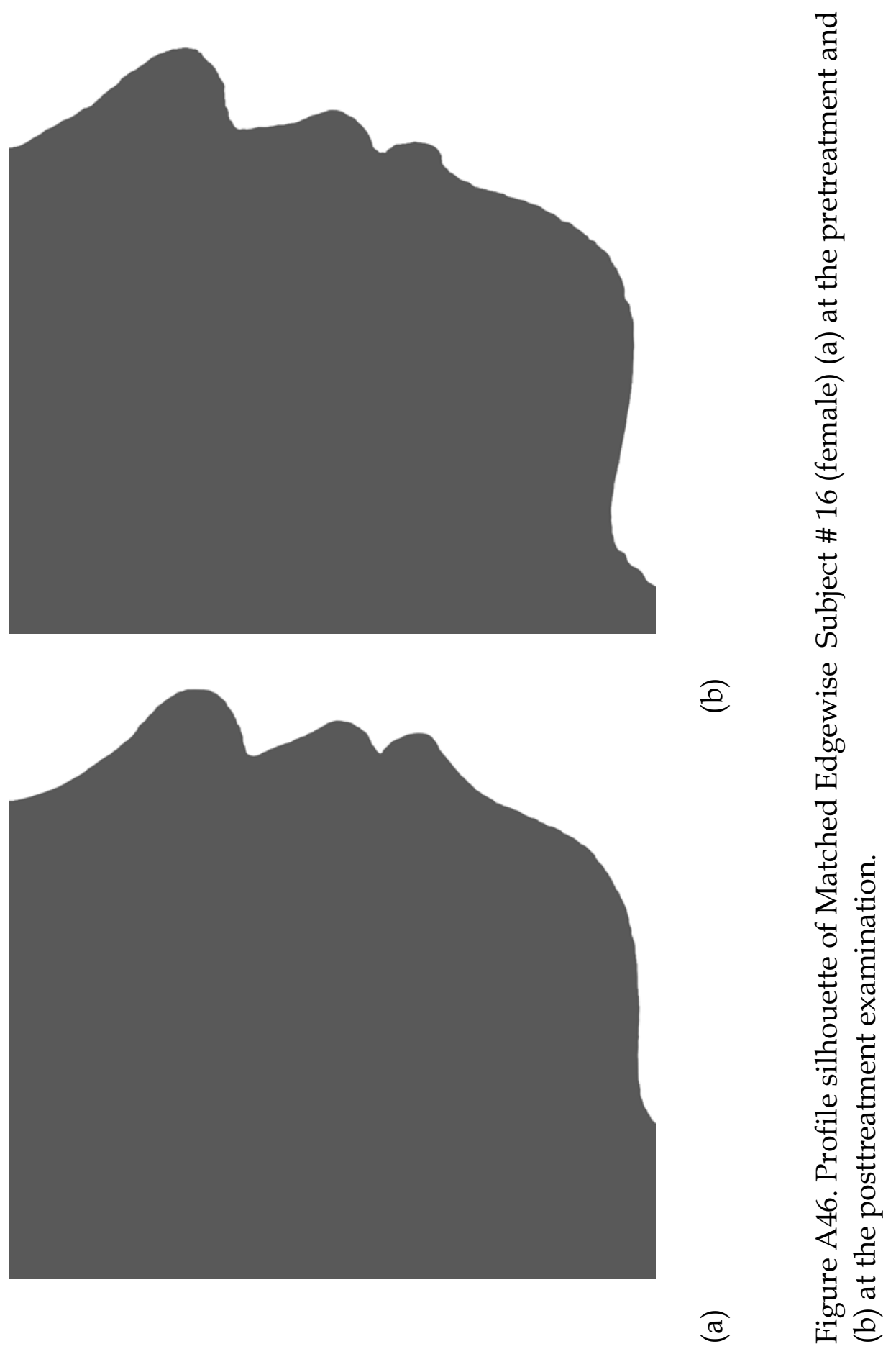




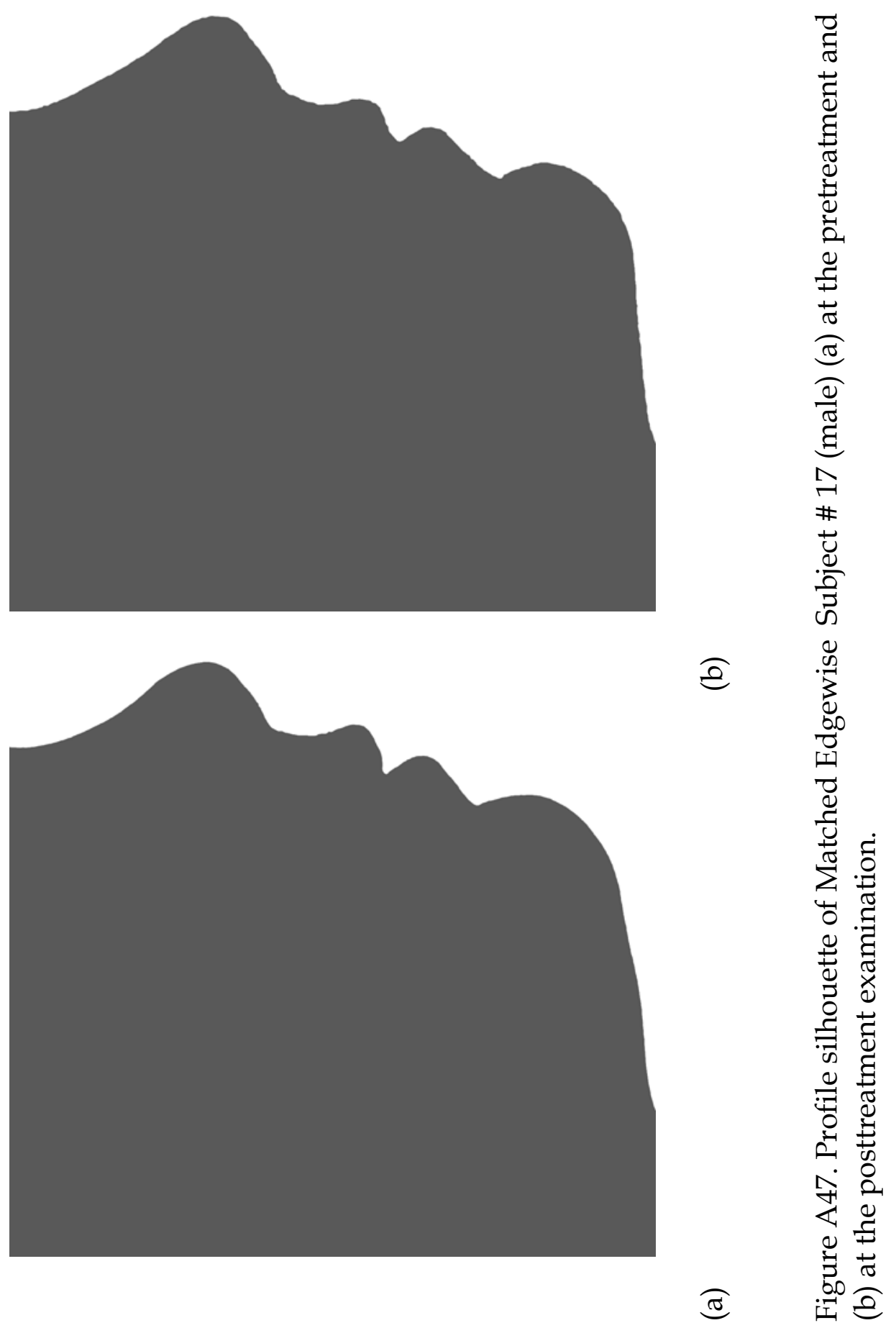




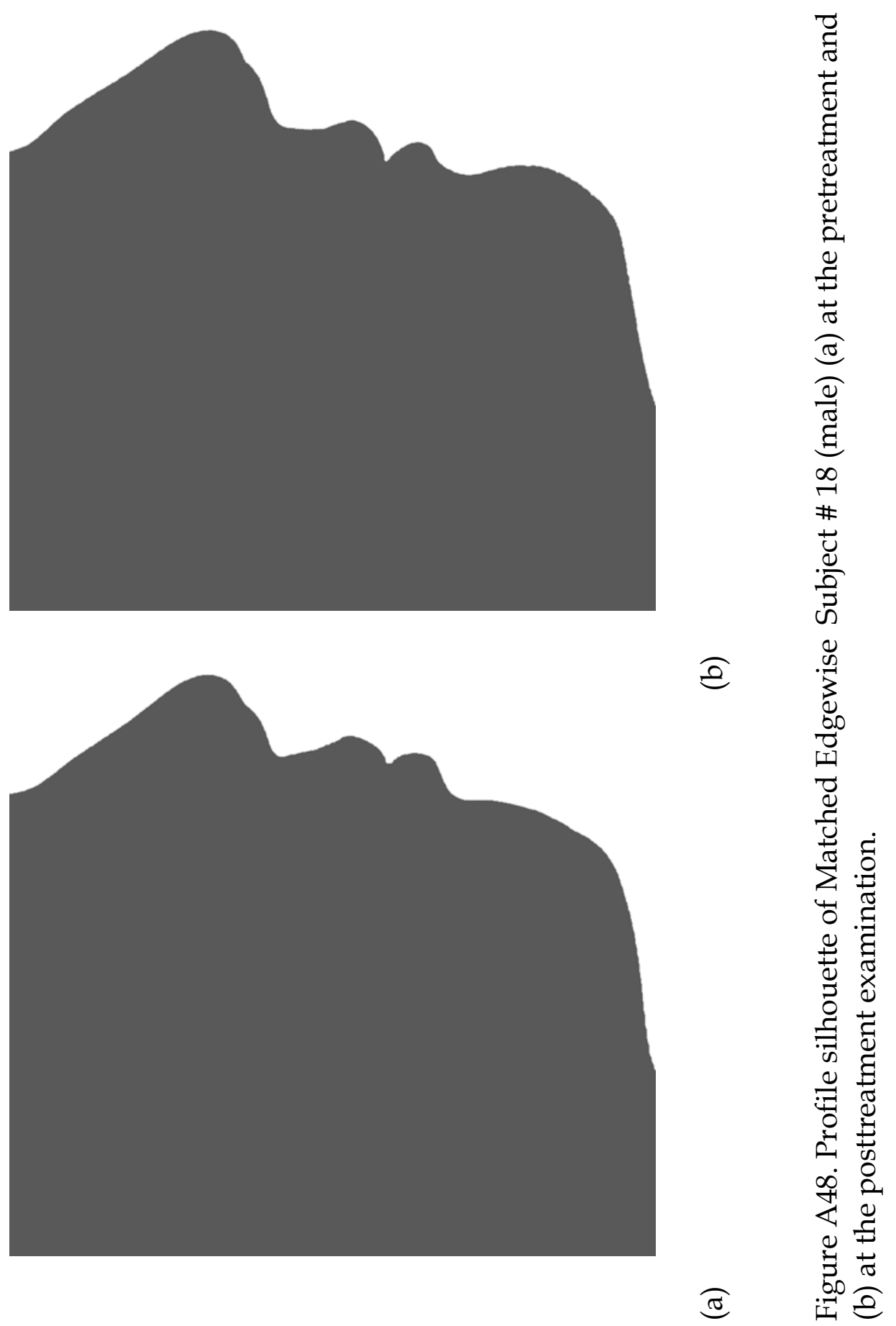




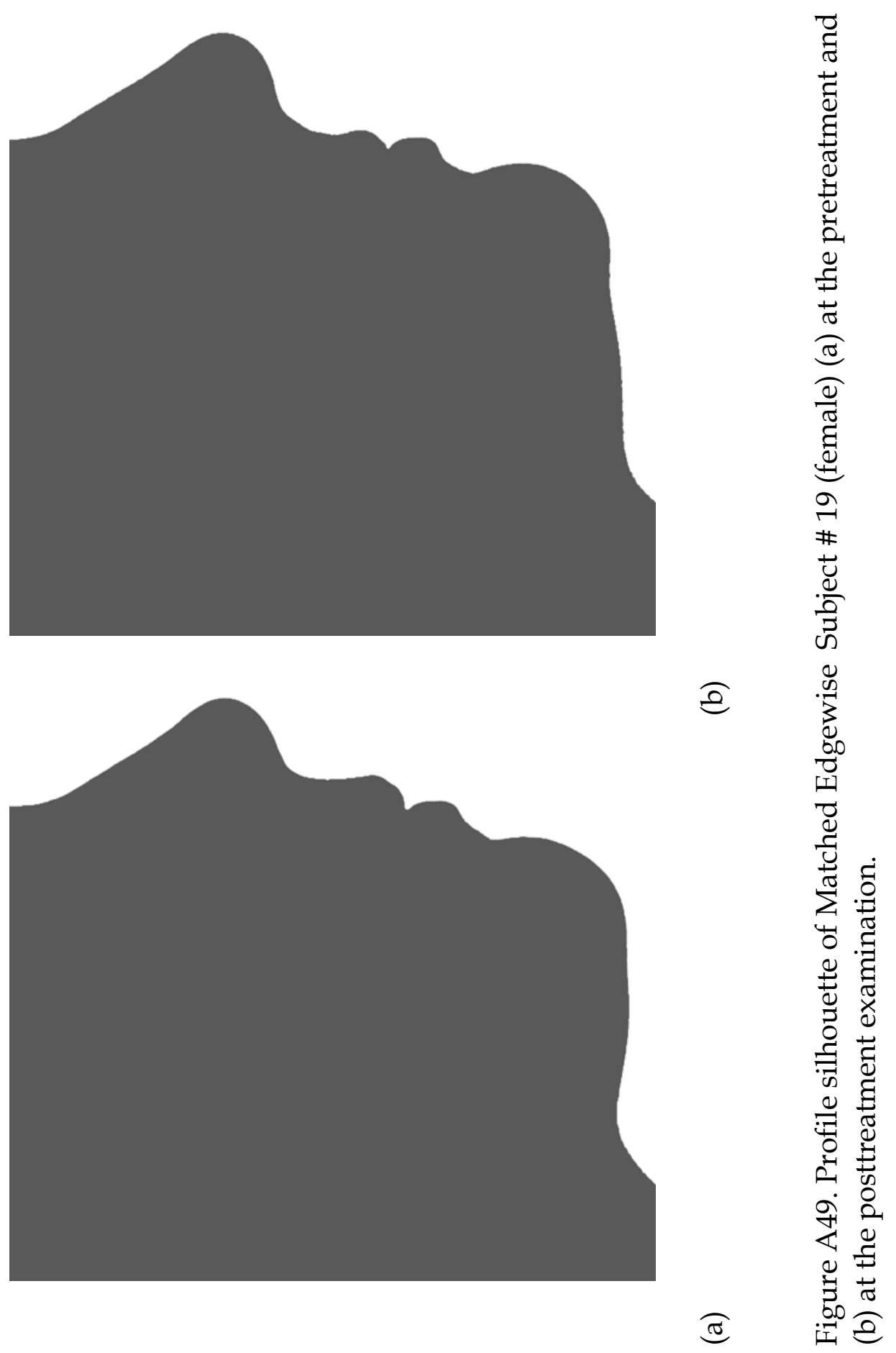




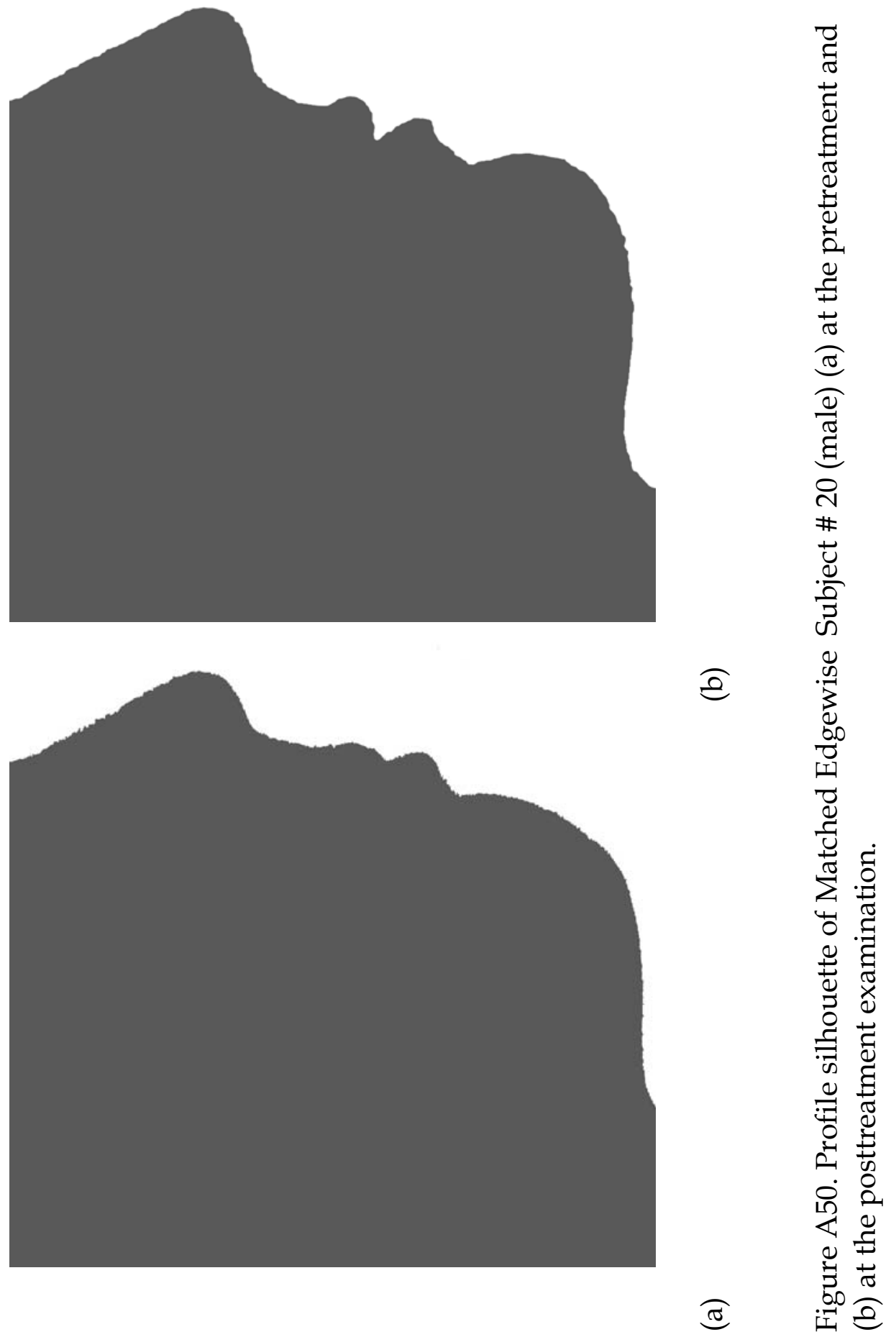




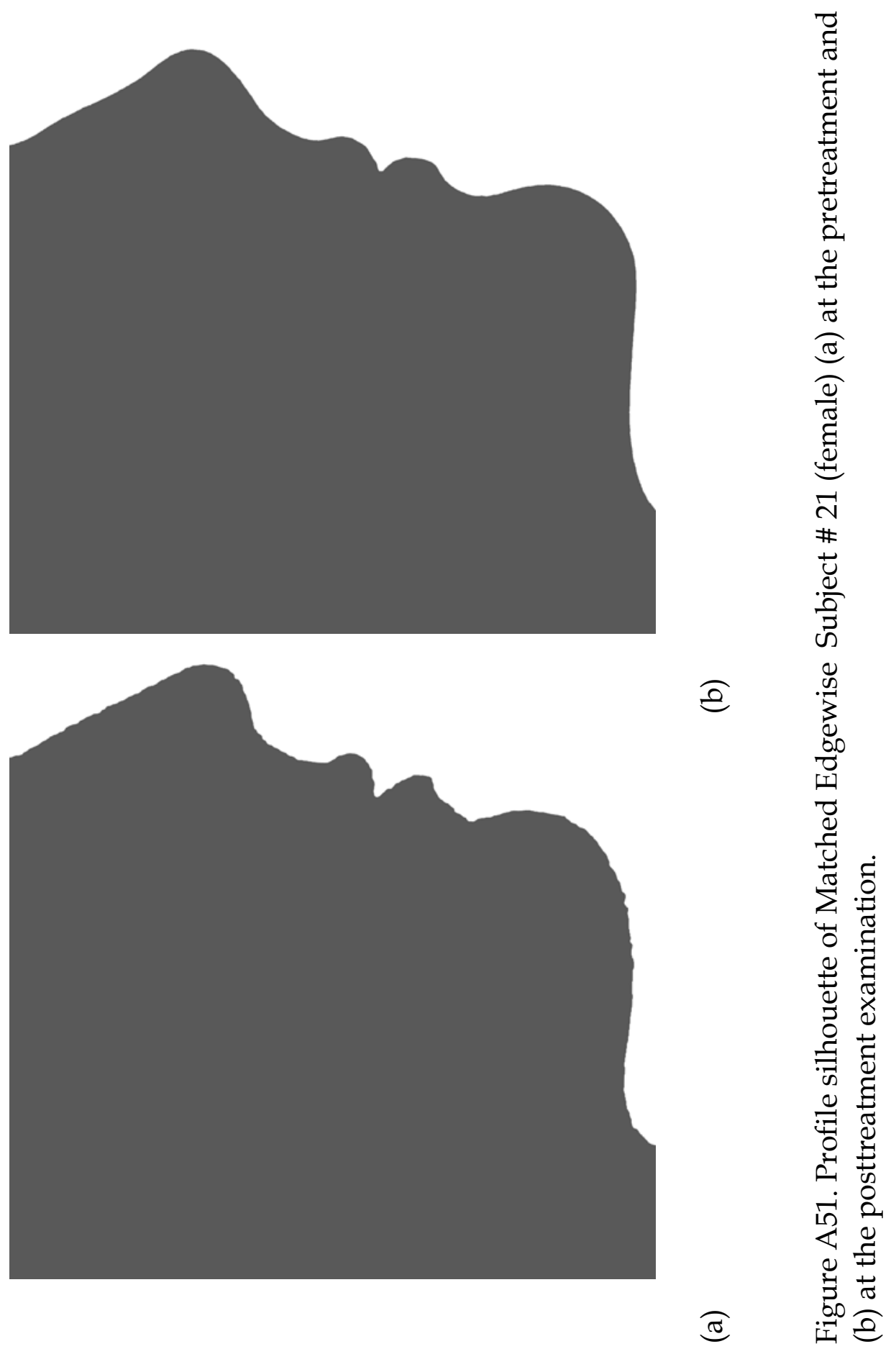




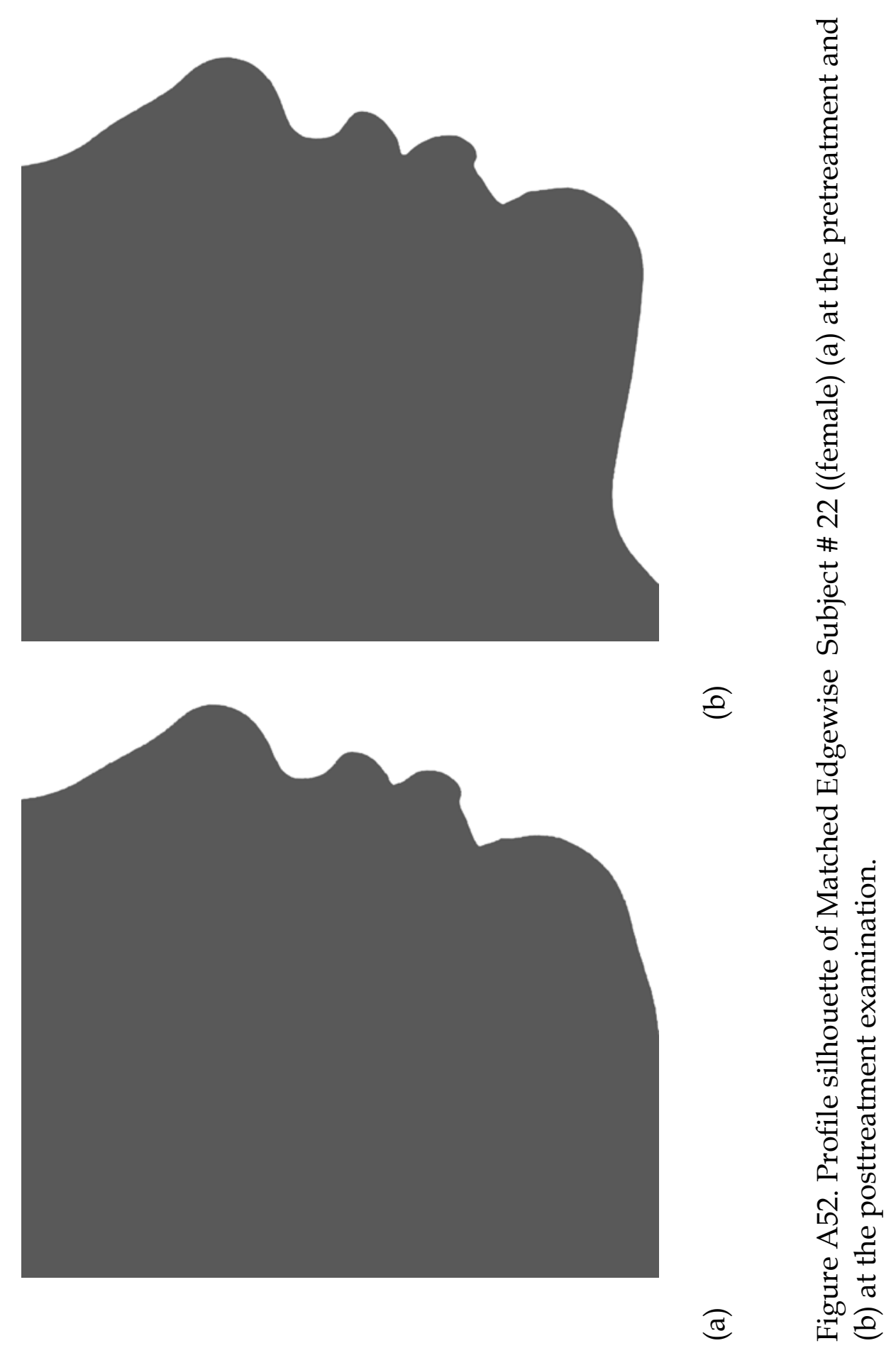




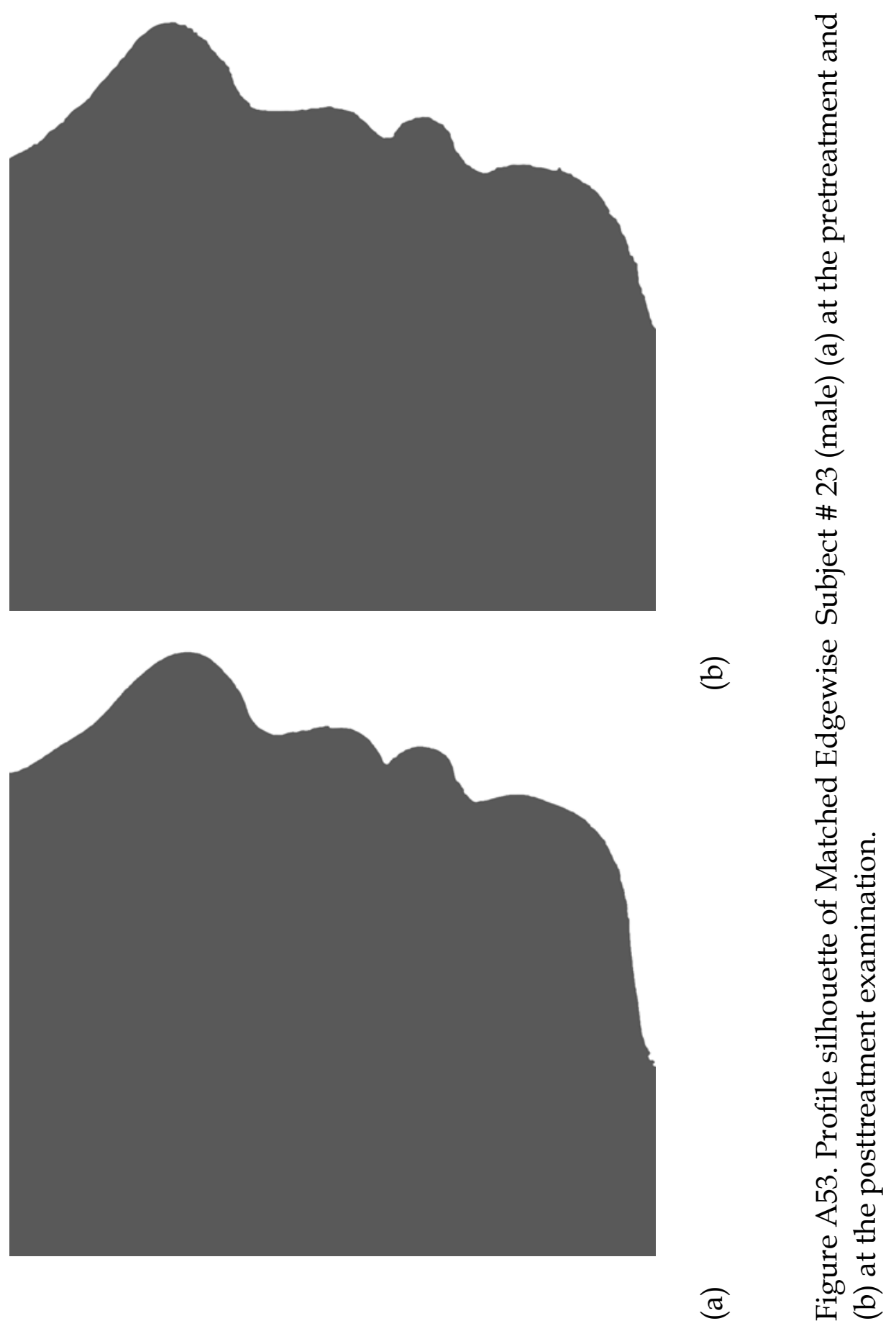




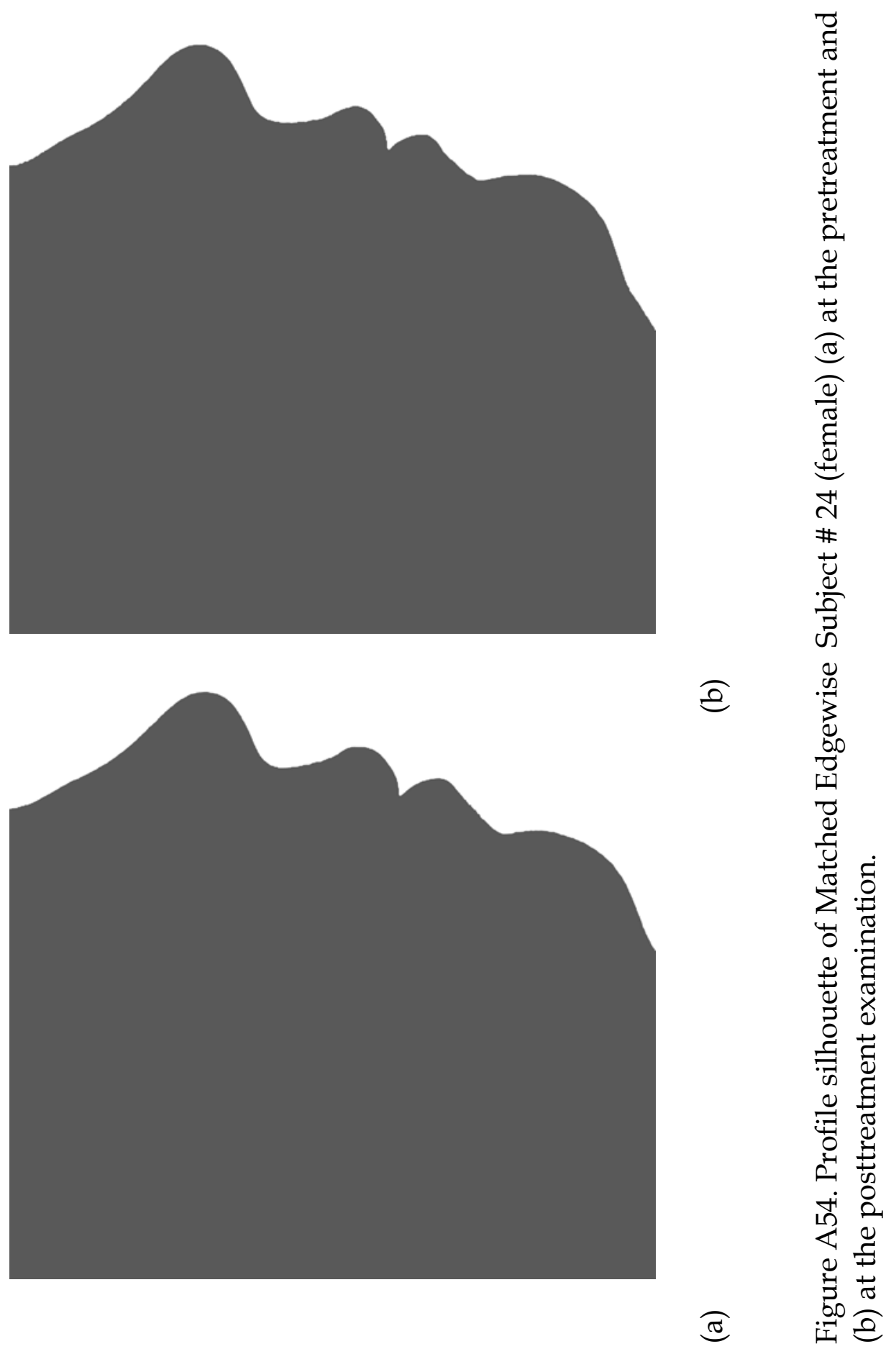




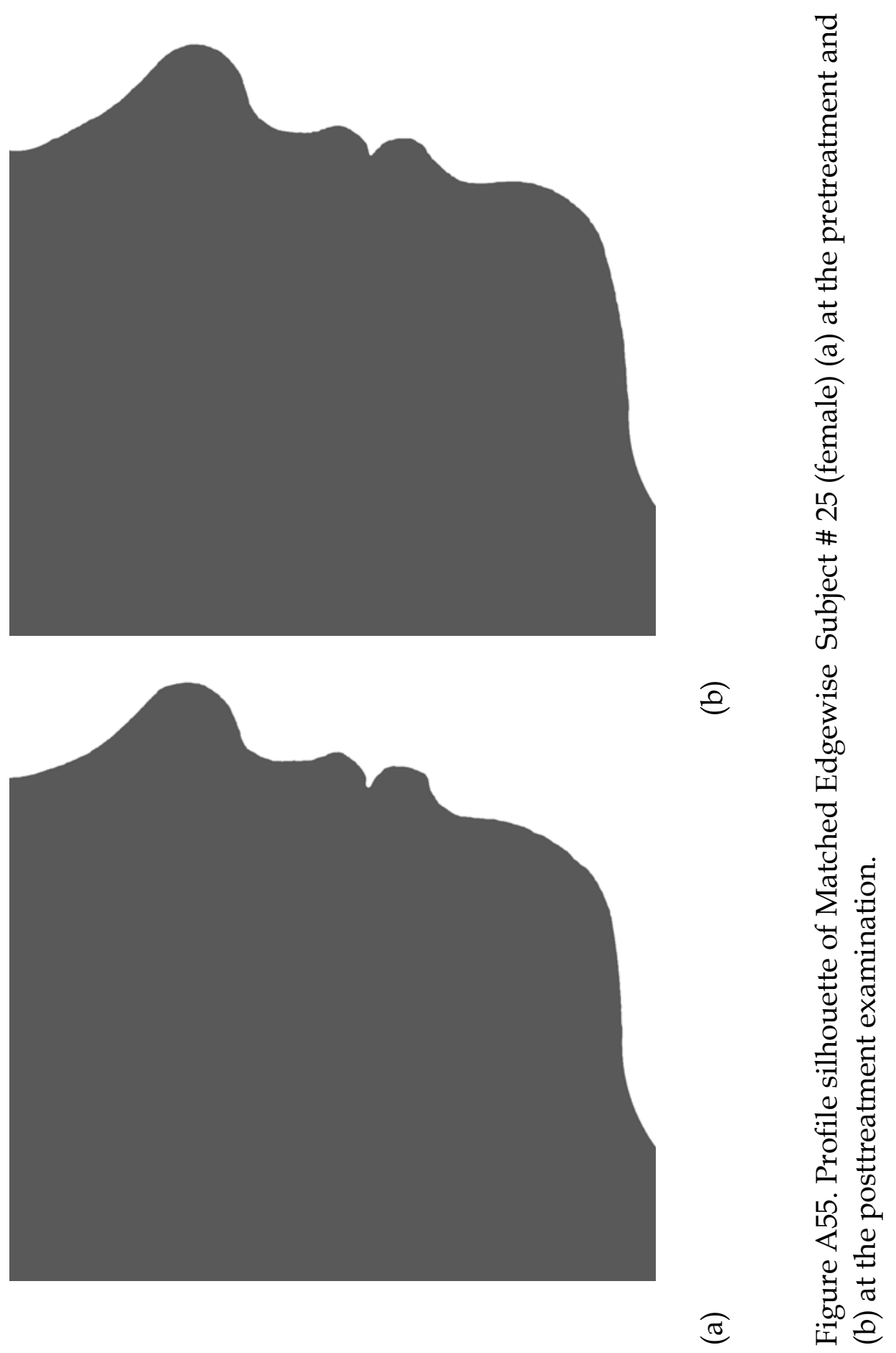




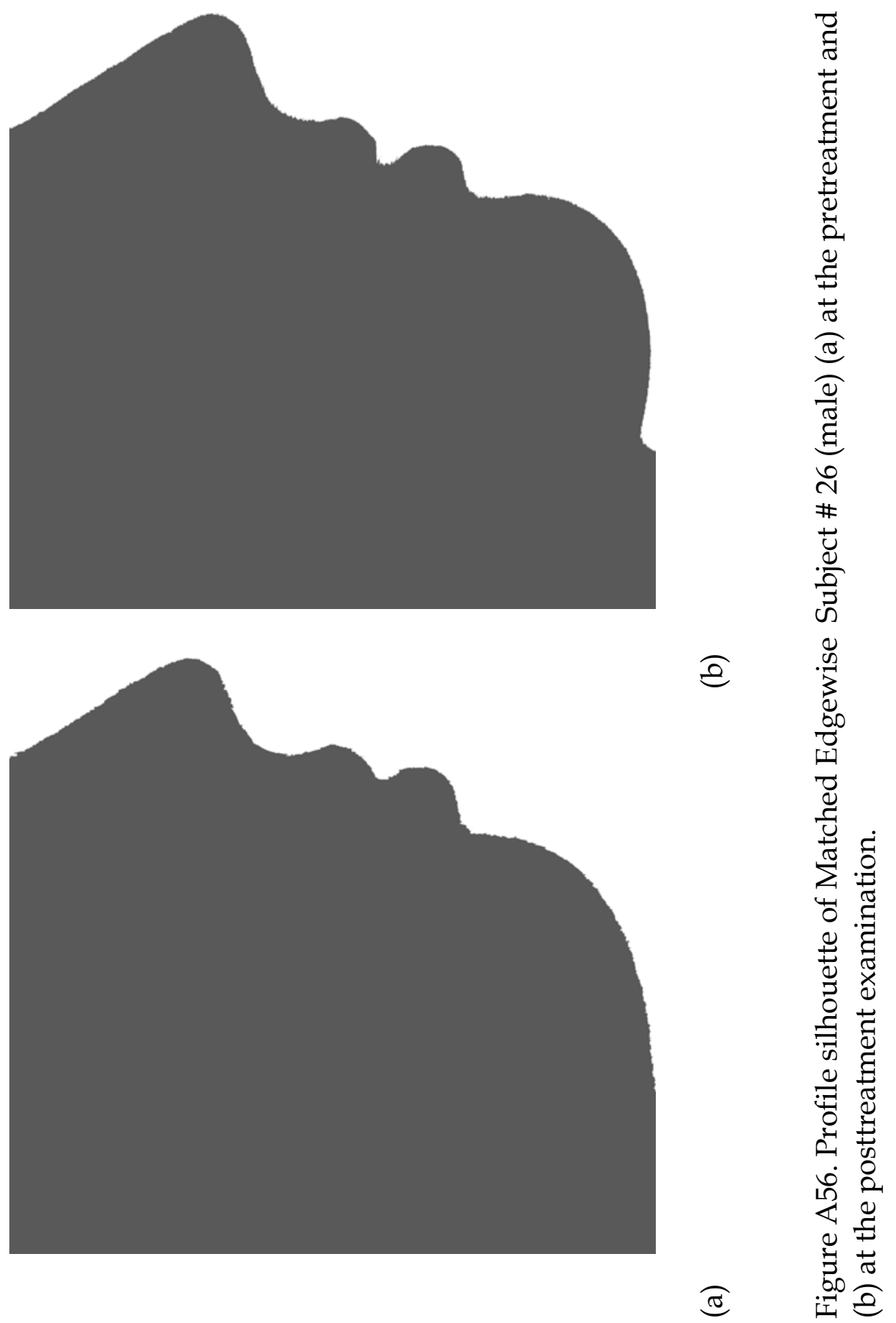




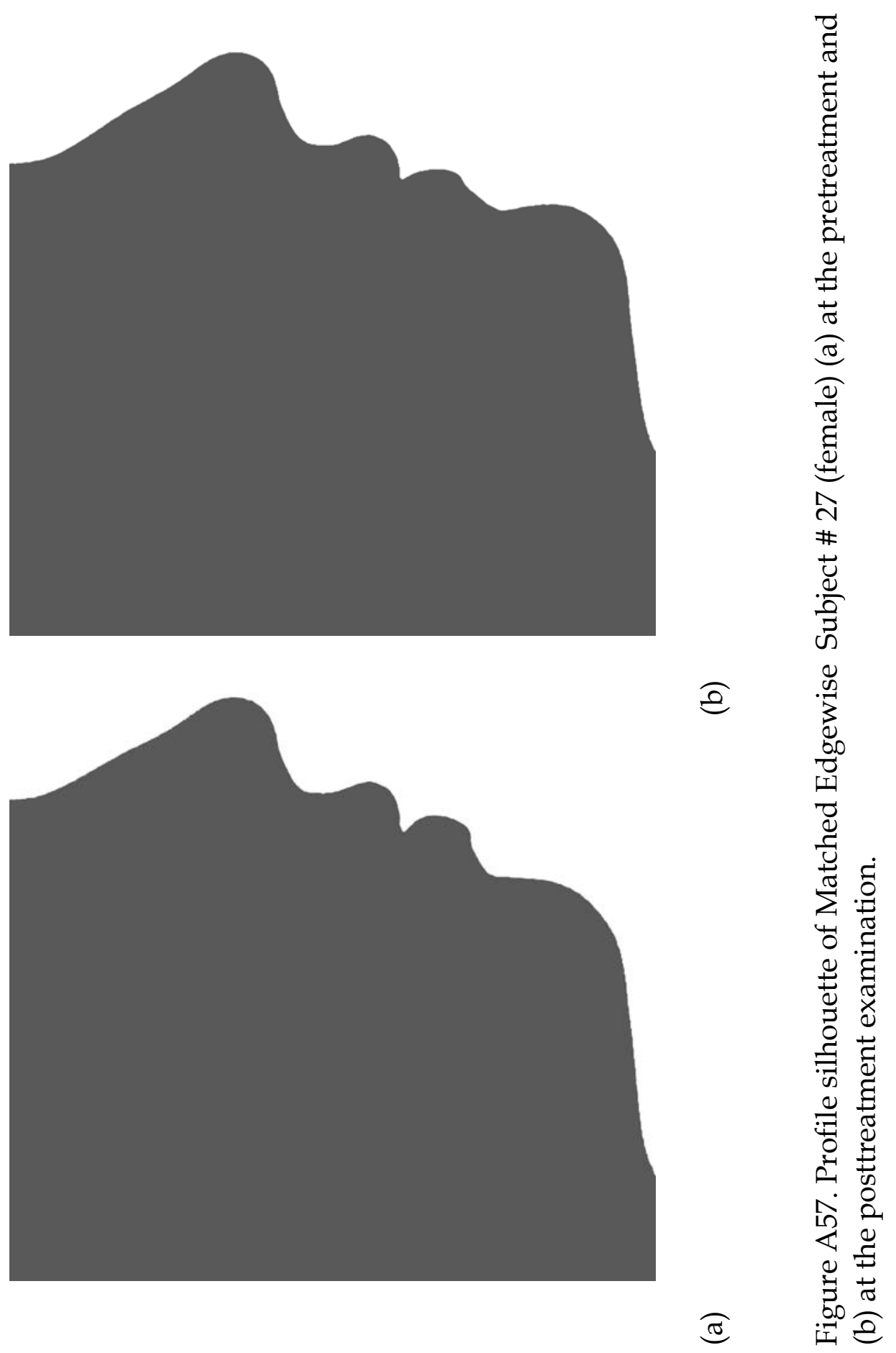




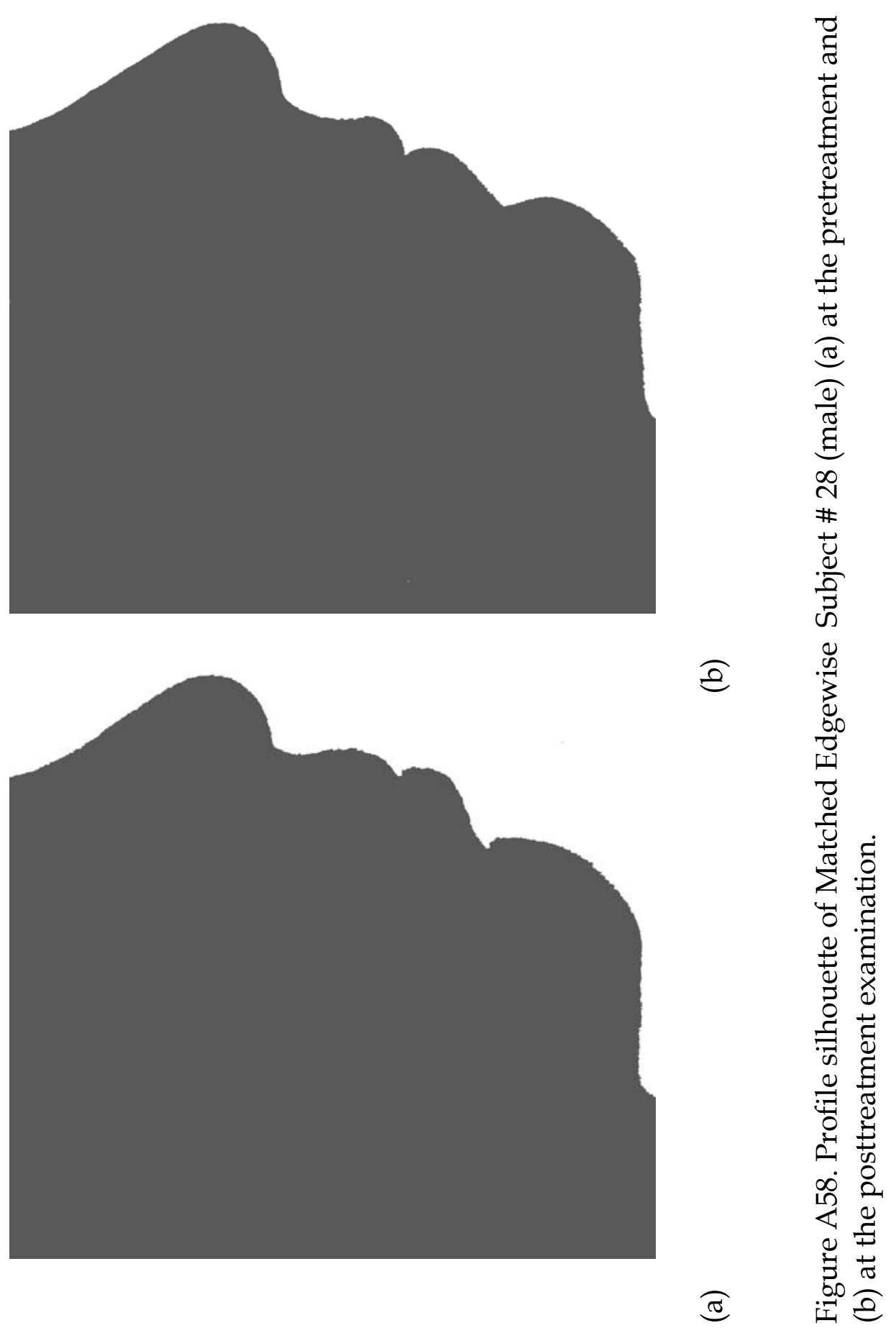




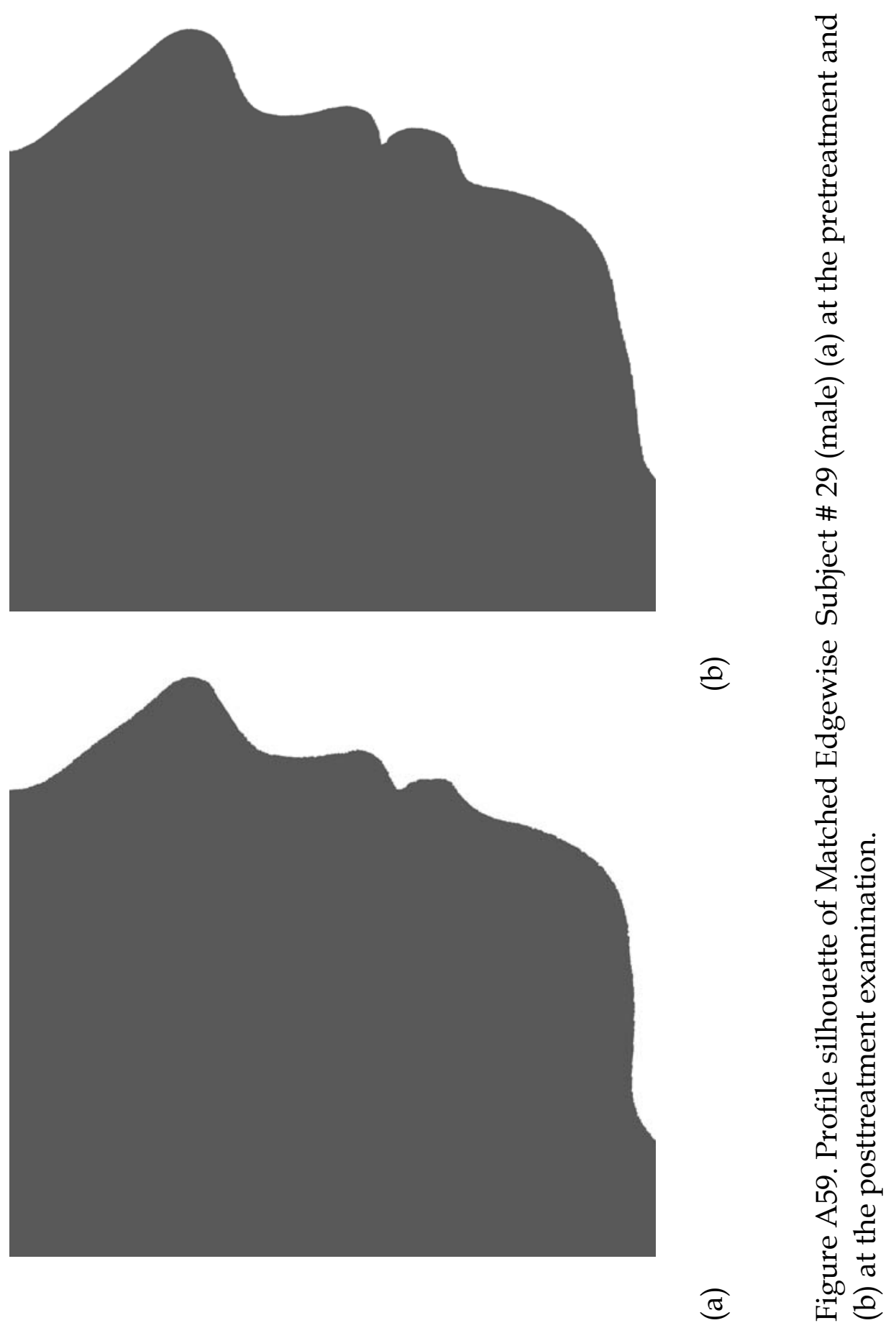




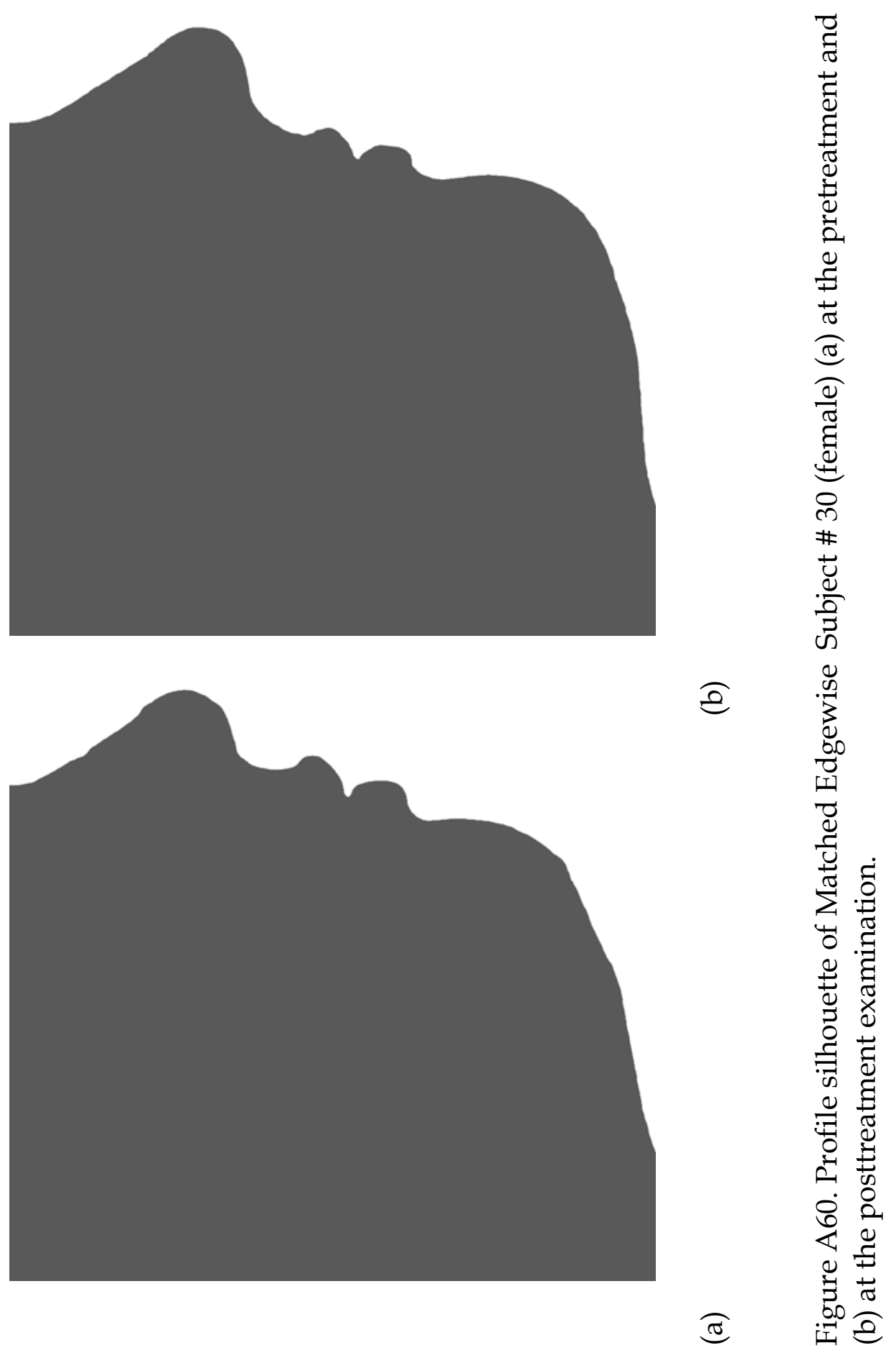




\section{VITA}

Kelly-Gwynne Mason Fergus, the daughter of Kenneth and Juliana Mason, was born in Nashville, Tennessee, on June 13, 1979. Kelly-Gwynne graduated from Springfield High School in 1997 and attended The University of Tennessee, Knoxville, where she received a Bachelor of Science degree in Business Administration, graduating Summa Cum Laude. She received her dental training and a Doctor of Dental Surgery degree from The University of Tennessee Health Science Center, Memphis, in May of 2005. In August of 2005, she entered The University of Tennessee Health Science Center as a graduate student in the Department of Orthodontics and is expected to receive her Master of Dental Science in May 2008. Kelly-Gwynne and her husband, Jay W. Fergus, plan to live in Jonesboro, Arkansas, upon graduation. 